Stephan Winters

\title{
Die kollektive Vorsorge für den Pflegefall im Alter
}

Eine Untersuchung am Beispiel der gesetzlichen Pflegeversicherung in den Niederlanden 


\section{Stephan Winters}

\section{Die kollektive Vorsorge für den Pflegefall im Alter}

Die Erfahrungen mit der gesetzlichen Pflegeversicherung in den Niederlanden haben in der deutschen Pflegedebatte nur wenig Beachtung gefunden. In dieser Arbeit werden sie erstmals umfassend beschrieben und analysiert. Zur Bewertung des Systems wird ein spezifischer Katalog sozialpolitischer und ökonomischallokativer Kriterien entwickelt. Unter anderem wird dabei die mögliche Bedeutung des moral hazard in der Pflegeversicherung ausführlich untersucht. Im Ergebnis erweist sich die niederländische Lösung in weiten Teilen als attraktives Modell. Eine vergleichende Kritik des deutschen Pflegeversicherungsgesetzes rundet die Studie ab.

Stephan Winters wurde 1963 geboren. Er studierte Volkswirtschaftslehre in Münster und Hamburg. Von 1990 bis 1994 war er Wissenschaftlicher Angestellter am Institut für Ausländisches und Internationales Finanz- und Steuerwesen an der Universität Hamburg und hat dort im Juni 1995 seine Promotion abgeschlossen. Seit 1994 arbeitet er als finanzpolitischer Referent bei der GAL-Fraktion (Bündnis 90/Die Grünen) in der Hamburgischen Bürgerschaft. 
Die kollektive Vorsorge für den Pflegefall im Alter

Eine Untersuchung am Beispiel der gesetzlichen Pflegeversicherung in den Niederlanden 


\section{FINANZWISSENSCHAFTLICHE SCHRIFTEN}

Herausgegeben von den Professoren

Albers, Krause-Junk, Littmann, Oberhauser, Pohmer, Schmidt

Band 73

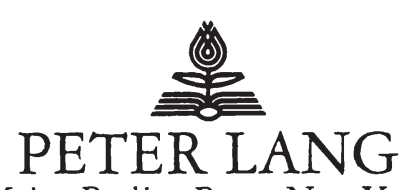

Frankfurt am Main · Berlin - Bern - New York · Paris - Wien 


\section{Stephan Winters}

\section{Die kollektive Vorsorge für den Pflegefall im Alter}

Eine Untersuchung am Beispiel der gesetzlichen Pflegeversicherung in den Niederlanden

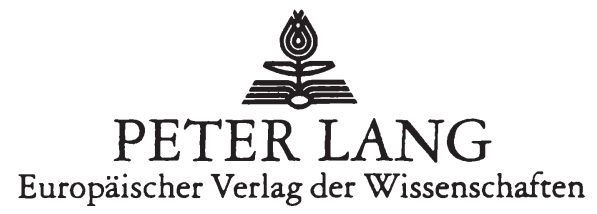


Die Deutsche Bibliothek - CIP-Einheitsaufnahme

Winters, Stephan:

Die kollektive Vorsorge für den Pflegefall im Alter : eine Untersuchung am Beispiel der gesetzlichen Pflegeversicherung in den Niederlanden / Stephan Winters. - Frankfurt am Main ; Berlin ; Bern ; New York ; Paris ; Wien : Lang, 1996

(Finanzwissenschaftliche Schriften ; Bd. 73)

Zugl.: Hamburg, Univ., Diss., 1995

ISBN 3-631-49707-5

NE: GT

Open Access: The online version of this publication is published on www.peterlang.com and www.econstor.eu under the international Creative Commons License CC-BY 4.0. Learn more on how you can use and share this work: http://creativecommons. org/licenses/by/4.0.

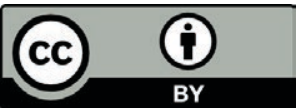

This book is available Open Access thanks to the kind support of ZBW - Leibniz-Informationszentrum Wirtschaft.

\author{
D 18 \\ ISSN 0170-8252 \\ ISBN 3-631-49707-5 \\ ISBN 978-3-631-75191-6 (eBook) \\ (C) Peter Lang GmbH \\ Europäischer Verlag der Wissenschaften \\ Frankfurt am Main 1996 \\ Alle Rechte vorbehalten.
}

Das Werk einschließlich aller seiner Teile ist urheberrechtlich geschützt. Jede Verwertung außerhalb der engen Grenzen des Urheberrechtsgesetzes ist ohne Zustimmung des Verlages unzulässig und strafbar. Das gilt insbesondere für Vervielfältigungen, Übersetzungen, Mikroverfilmungen und die Einspeicherung und Verarbeitung in elektronischen Systemen.

Printed in Germany 123457 
Mit Dank an alle,

die beteiligt oder betroffen waren... 
Stephan Winters - 978-3-631-75191-6

Downloaded from PubFactory at 01/11/2019 07:17:47AM

via free access 


\section{Inhalt}

1. Einführung

1.1 Motivation

1.2 Vorüberlegungen zum Umgang mit dem Untersuchungsgegenstand

1.3 Überblick

2. Der Pflegefall als ungedecktes Risiko

2.1 Das Risiko, pflegebedürftig zu werden: Eine Umschreibung

2.2 Gründe für die geringe Verbreitung privater Pflegeversicherungen

2.2.1 Angebotsseitige Gründe

2.2.2 Subjektive Gründe auf seiten der Nachfrage

2.2.3 Die garantierte Mindestsicherung als Grund für die Nachfrageschwäche

2.3 Gründe für das Fehlen einer Sozialversicherung in bezug auf das Pflegerisiko

3. Das Ziel und die Maßstäbe einer gesetzlichen Regelung zur Absicherung des Pflegerisikos

3.1 Vorbemerkung zum generellen Ziel und zu seiner Begründung

3.2 Zieldimensionen

3.2.1 Reichweite in bezug auf den versicherten Personenkreis 
3.2.2 Leistungsgrenzen: Leistungszugang und Selbstbeteiligung

3.2.3 Leistungsinhalte und Leistungsform

3.2.4 Leistungsqualität

3.3 Spezifikationen und Änderungen am Zielkatalog bei Einstufung der Pflegeleistungen als Grundbedarfsgüter

4. Effizienzkriterien

4.2 Effizienz in Hinblick auf die Finanzierung der Pflegesicherung

4.2.1 Das Kriterium der Verläßlichkeit und seine Folgen für die Wahl der Finanzierungsquelle

4.2.2 Das Äquivalenzkriterium und seine Folgen für die Bewertung des einkommensabhängigen Beitrags

4.2.2.1 Der Grundgedanke: Mindestsicherung und Beitragsäquivalenz

4.2.2.2 Die Einkommensabhängigkeit des Beitrags -eine Spezifikation

4.2.2.3 Ergänzende Überlegungen zur Frage eines Staatszuschusses

4.2.3 Die Wahl des Finanzierungsmodus im Lichte beider Kriterien

4.3 Effizienz in Hinblick auf die Gefahr des moral hazard

4.3.2 Moral hazard seitens der Versicherten selbst

4.3.3 Moral hazard seitens der Angehörigen Pflegebedürftiger

4.3.3.1 Einleitung

4.3.3.2 Die vermutete "Abschiebung" ins Pflegeheim als Kernproblem

100

4.3.3.3 Zur juristischen Frage der familialen Pflegepflicht

4.3.3.4 Zur theoretischen Spannung zwischen Nutzenkalkül und Familienidyll 
5. Die gesetzliche Pflegeversicherung in den Niederlanden

5.1 Die Entstehungsgeschichte des AWBZ und seine ursprüngliche Form

5.2 Eine kleine Chronik wesentlicher Veränderungen im System der Pflegeversicherung bis Anfang der neunziger Jahre

5.3 Die derzeitige Gesetzeslage im einzelnen

5.4 Die Entwicklung der Leistungsmengen und -preise im Überblick

5.4.1 Stationärer Sektor

5.4.2 Ambulanter Sektor

5.5 Aktuelle Tendenzen und Perspektiven künftiger Entwicklung

5.5.1 Die Reform des Krankenversicherungswesens in den Niederlanden

5.5.1.1 Das Reformprojekt im Überblick: Gründe, Ziele, Konzepte

5.5.1.2 Zum Stand und Stillstand der Reform

5.5.1.3 Auswirkungen im Bereich der Pflegeversicherung

5.5.2 Substitution als Antwort auf demographischen Druck

6. Zur Bewertung der gesetzlichen Pflegeversicherung in den Niederlanden

6.1 Zur Bewertung des Systems unter Zielgesichtspunkten

6.1.1 Vorbemerkung in Hinblick auf erklärte Ziele 
6.1.3 Leistungszugang 165

$\begin{array}{ll}\text { 6.1.4 Selbstbeteiligung } & 174\end{array}$

6.1.5 Leistungsinhalte und Leistungsform 177

6.1.6 Leistungsqualität 182

6.2 Pflege als Grundbedürfnis - zur Bewertung anhand des modifizierten Zielkatalogs $\quad 188$

6.3 Zur Bewertung unter Effizienzgesichtspunkten 189

6.3.1 Verläßlichkeit 189

6.3.2 Finanzierungsäquivalenz 192

6.3.3 Moral Hazard 197

6.3.3.1 Vorüberlegungen 197

6.3.3.2 Ein Heimquotenvergleich 200

7. Zum Vergleich: Eine Kritik der künftigen Pflegesozialversi-

cherung in der Bundesrepublik Deutschland

208

7.1 Zur Bewertung unter Zielgesichtspunkten 208

$\begin{array}{lll}7.2 \text { Zur Bewertung unter Effizienzgesichtspunkten } & 218\end{array}$

8. Zusammenfassung: Fünfzehn Thesen zum Schluß 226

$\begin{array}{ll}\text { Abkürzungen } & 229\end{array}$

Literatur

231 


\section{Einführung}

\subsection{Motivation}

In der Bundesrepublik Deutschland ist die Diskussion um eine kollektiv verbindliche Sicherung gegen das Risiko der Pflegebedürftigkeit ungefähr so alt wie in den Niederlanden die entsprechende gesetzliche Regelung. Während also dort auf Erfahrungen aus rd. 25 Jahren zurückgeblickt werden kann, ist hierzulande erst im Jahre 1994 die Entscheidung gefallen, eine gesetzliche Pflegeversicherung in den beiden Folgejahren stufenweise einzuführen.

Der Gedanke, die im Nachbarland gesammelten Erfahrungen in Hinblick auf die Ausgestaltung der Pflegeversicherung hierzulande auszuwerten und ggfs. nutzbar zu machen, ist naheliegend und nicht ganz neu. Daß ein solcher Versuch mit der vorliegenden Arbeit unternommen wird, ist aus folgenden Überlegungen motiviert:

- Der bereits vorliegende Transfer, also deutsch- oder englischsprachige Texte über die niederländische Versicherungslösung und deren Resultate, ist weder umfangreich noch inhaltlich erschöpfend. Es handelt sich ausschließlich um kleinere Aufsätze oder um Einzelkapitel breiter angelegter Vergleichsstudien, die sich in überschaubarer Anzahl über einen Erscheinungszeitraum von rd. 15 Jahren verteilen. Das somit in Deutschland zugängliche Sachwissen ist schon auf der deskriptiv-empirischen Ebene lückenhaft und überholt.

- $\quad$ Mit dieser brüchigen Informationsgrundlage korrespondiert ein fahrlässiger Umgang mit isolierten und oftmals irreführenden Einzeldaten, wie sie mit einer gewissen Kontinuität durch die deutsche Pflegedebatte geistern. Zugespitzt könnte man von einer Tendenz zur Mythenbildung sprechen, die im Regelfall zum Beleg für die Untauglichkeit einer Sozialversicherungslösung, mitunter auch dem entgegengesetzten Interesse dient. Auch einer solchen Mißbrauchstendenz kann bereits durch eine gründliche Darstellung der niederländischen Verhältnisse entgegengewirkt werden.

- Weiterhin erscheint es gerade in Hinblick auf eine mögliche Verwertung niederländischer Erfahrungen wünschenswert, diese nicht nur zu erfassen und zur Kenntnis zu nehmen, sondern auch analytisch zu durchdringen und in den Kontext theoretischer Überlegungen und Kontroversen einzubetten. Im Erfolgsfall kann ein solches Unterfangen zum genaueren Verständnis des Pfle- 
geproblems als eines eigenständigen Objekts der Diskussion um die Sozialversicherung beitragen. Dies könnte eine Bereicherung der wissenschaftlichen Debatte bedeuten, die bislang vielfach von - in ihrer Übertragbarkeit teilweise unzureichend reflektierten - theoretischen Anleihen aus den Forschungsfeldern der Kranken- und der Rentenversicherung beherrscht wird. Eine solche gezielte Analyse liegt meines Wissens in den Niederlanden nicht vor. Sehr wohl aber gibt es dort - in weit höherem Maße als in der Bundesrepublik - für den gesamten Pflegebereich eine Fülle empirischer Daten und Detailstudien, deren sich ein solches Projekt bedienen kann.

- Schließlich berührt die Auseinandersetzung mit der Pflegeversicherung zumindest schlaglichtartig einige grundsätzlichere Fragen der sozialen Sicherung. Dies reicht von deren genereller Begründung über ihre Tragfähigkeit angesichts zunehmender Finanzierungsprobleme bis hin zu den Formen, die sie konzeptionell annehmen kann, empirisch angenommen hat und gemäß gewisser Zielvorstellungen annehmen soll. Gerade in der Bundesrepublik mangelt es der herrschenden Debatte nach meinem Eindruck immer noch an konstruktiven Versuchen, gesellschaftlich begründbare Ziele sozialer Sicherung zu identifizieren und von dort aus unter Verwendung ökonomischer Erkenntnisse zu Gestaltungs- bzw. Reformvorschlägen in bezug auf real bestehende Systeme zu gelangen. Die Arbeitsteilung und Kooperation zwischen Ökonomie und Sozialpolitik funktioniert offenbar nur unzulänglich.

Zuletzt sind aktuelle Entwicklungen sowohl in den Niederlanden als auch in Deutschland zu nennen, die die Beschäftigung mit dem Thema der Arbeit lohnend erscheinen lassen:

Erstens ist die Pflegeversicherung in den Niederlanden mitbetroffen von der dort in den letzten Jahren schrittweise in Gang gesetzten Reform des Krankenversicherungswesens, die zumindest der Idee nach tiefgreifender ist als die inzwischen auch in Deutschland eingeleiteten Veränderungen. Die damit verbundenen Neuerungen sind zwar nicht pflegespezifisch, aber mit dem Untersuchungsgegenstand insofern eng verbunden, als die Sozialversicherung, deren Eignung zur Bewältigung des Pflegerisikos ja zur Debatte steht, in wichtigen Punkten ein neues Gesicht erhält. Auch im Übertrag auf die Bundesrepublik liegt der Zusammenhang auf der Hand: Nicht nur sieht das soeben beschlossene Konzept die organisatorische Anbindung der Pflegeversicherung an die GKV vor; vielmehr wird auch finanziell und politisch der Spielraum für den 
einzuführenden Versicherungszweig von den Entwicklungen und Reformerfolgen im Bereich des Krankenversicherungswesens maßgeblich bestimmt sein. Insofern erscheint es sinnvoll, die Betrachtung und Analyse der niederländischen Verhältnisse zum Zweck der Gewinnung von Anregungen für den deutschen Reformprozeß zumindest ansatzweise auf das neue Krankenversicherungssystem auszuweiten. In dem Maße, wie sie den Pflegebereich und seine Finanzierung direkt betreffen, ist eine Berücksichtigung der Reformschritte ohnehin geboten.

Zweitens ist damit bereits angedeutet, daß die Auseinandersetzung um die Pflegeversicherung in der Bundesrepublik mit dem Beschluß, sie im Rahmen der Sozialversicherung einzuführen, nicht wirklich beendet ist. Während sich die politische Debatte zuletzt auf Nebenschauplätze wie die Frage der Kompensation zugunsten der Arbeitgeber verlagert oder um Details der diffizilen Kompromißfindung zwischen den Parteien gedreht hat, stehen sich Befürworter und Gegner des Gesamtprojekts "Gesetzliche Pflegeversicherung" nach wie vor gänzlich unversöhnt gegenüber. Was sie einzig verbindet, ist die kritische Haltung gegenüber dem verabschiedeten Gesetz, das den einen als unzulängliche "Mogelpackung " ${ }^{1}$, den anderen als "schwerwiegendste Fehlentscheidung seit Jahrzehnten"2 gilt. Daher wird schon die bevorstehende Einführungsphase der Versicherung von neuerlichen Kontroversen begleitet sein, die spätestens dann offen aufbrechen dürften, wenn erstmals eine Anhebung des gesetzlich fixierten Beitragssatzes zur Debatte steht. Eine an den Zielen der Versicherung ausgerichtete Aufbereitung und Auswertung des niederländischen Modells als der einzigen real existierenden Sozialversicherung für den Pflegefall kann auch in diesem Kontext ein Beitrag zur Versachlichung sein.

\subsection{Vorüberlegungen zum Umgang mit dem Untersuchungsgegenstand}

Die wissenschaftliche Behandlung der Pflegeproblematik und auf diese bezogener Sicherungsmöglichkeiten im Rahmen einer ökonomischen Arbeit setzt Überlegungen zur Einordnung des Untersuchungsgegenstandes voraus, die mit methodologischen Fragen verknüpft sind. Es ist also erstens das Verhältnis des Pflegerisikos zum

\footnotetext{
${ }^{1}$ J. Allemeyer (1993), S. 1

2 So die Bundesvereinigung der deutschen Arbeitgeberverbände laut Süddeutsche Zeitung vom 11.03.1994.
} 
Objektbereich einer Politik der sozialen Sicherung zu erörtern; zweitens gilt es im Lichte einer solchen Klassifikation herauszuarbeiten, wie, inwieweit und in welcher Absicht das vorliegende Problem einer ökonomischen Betrachtung unterzogen werden kann.

a) Pflegesicherung als Sozialpolitik ?

Nach LAMPERT ${ }^{3}$ ist "Sozialpolitisches Handeln (...) darauf gerichtet (..),

1. die wirtschaftliche und soziale Stellung von wirtschaftlich und/oder sozial absolut oder relativ schwachen Personenmehrheiten im Sinne der in einer Gesellschaft verfolgten gesellschaftlichen und sozialen Grundziele zu verbessern und

2. die wirtschaftliche und soziale Stellung von wirtschaftlich und/oder sozial schwachen Personenmehrheiten für den Fall des Eintritts existenzgefährdender Risiken zu sichern."

Wenngleich sehr abstrakt, enthält diese Bestimmung doch die beiden wesentlichen Funktionsmuster, unter denen sich sozialpolitisches Geschehen ${ }^{4}$ fassen läßt. Es sind dies zum einen die Umverteilung (im weiten Sinn, also nicht nur in Form monetärer Transfers) und zum andern die Risiken mildernde Sicherung. Gleichzeitig macht die Definition aber deutlich, daß diese Begriffe kein überschneidungsfreies Ordnungsschema ergeben können, da der Sicherungsaspekt ja auf "schwache" Gruppen beschränkt wird, also seinerseits Umverteilung impliziert. Sofern man berücksichtigt, daß umgekehrt auch die Zugehörigkeit zu einer "Schwachen Personenmehrheit" als sicherungsfähiges Risiko eingestuft werden kann ${ }^{5}$, wird die Unterscheidung vollends prekär. Allenfalls trennt sie die beiden Seiten derselben Medaille, indem Punkt 1 auf den Ausgleich bereits eingetretener, Punkt 2 auf den Ausgleich erst drohender Nachteile zielt.

Was nun die Einordnung der Pflegebedürftigkeit in das so definierte Feld der Sozialpolitik betrifft, so ist diese zweifelsfrei als "existenzgefährdendes Risiko" einzustufen. Wenn benötigte Pflege unterbleibt, ist die physische Existenz bedoht, wenn sie stattfindet, bedrohen ihre Kosten in vielen Fällen die "ökonomische Existenz", es droht Verarmung. Im Sinne von Punkt 2 der obigen Definition liegt sozialpolitisches Handeln

\footnotetext{
${ }^{3}$ H. Lampert (1991), S. 12

${ }^{4}$ Dieser Sprachgebrauch ist ebenfalls von LAMPERT inspiriert, der den Terminus "Sozialpolitik" für die Wissenschaft vom hier zu bestimmenden Gegenstandsbereich reserviert (ebd.).
}

${ }^{5}$ vgl. R. Eisen (1988), S. $126 \mathrm{f}$. 
im Bereich der Pflegesicherung also dann vor, wenn die betreffenden Regelungen das Ziel verfolgen, gerade für sozial Schwache einen Schutz vor den Folgen von Pflegebedürftigkeit zu gewährleisten. Die niederländische Pflegesozialversicherung erfüllt dieses Kriterium im Grundsatz ebenso wie das deutsche Konzept.

Im Sinne von Punkt 1 ist pflegepolitisches Handeln weiterhin dann als sozialpolitisch anzusehen, wenn es darauf zielt, die Lage bereits Pflegebedürftiger als benachteiligter Gruppe zu bessern. Auch dies ist bei den behandelten Konzepten mehr oder weniger stark bzw. ausdrücklich der Fall. Es ist somit gezeigt, daß es sozialpolitische Phänomene sind, die im folgenden zur Debatte stehen. Dies mag trivial erscheinen, ist aber für das weitere Vorgehen von einiger Bedeutung.

b) Vom ökonomischen Umgang mit sozialpolitischen Tatbeständen

Als "Sozialpolitik" bezeichnet LAMPERT die Wissenschaft von den oben definierten Dingen und stellt klar, daß es "verfehlt" wäre, "sie als eine wirtschaftswissenschaftliche Disziplin aufzufassen". ${ }^{6}$ Nach seiner Überzeugung "reichen die Methoden der Wirtschaftswissenschaften zu einer vollständigen Erfassung, Analyse und Lösung sozialpolitischer Probleme nicht aus". Dieses Verdikt wird damit begründet, daß auf der Ebene der Forschungsfelder "die Wirtschaftsordnung und die Sozialordnung Subsysteme der Gesellschaftsordnung sind, die logisch auf derselben Ebene angesiedelt und sozusagen 'gleichberechtigt' sind "7 Unter Ökonomen herrscht in diesem Punkt, mit unterschiedlicher Ausdrücklichkeit und Entschiedenheit, eine andere Sicht vor, die alles Sozialpolitische als "residual" (TITMUSS) begreift. Eine solche Haltung klingt etwa in der Frage BERTHOLDs durch, "welche Aufgabe die Sozialpolitik in Gesellschaften hat, die sich grundsätzlich für eine marktwirtschaftliche Ordnung entschieden haben". ${ }^{8}$ Noch deutlicher wird VAUBEL, wenn er die Aufgabe des Ökonomen bei der "Beurteilung eines Systems der sozialen Sicherung" u.a. darin erblickt, "das bestehende System mit dem ökonomisch gerechtfertigten (zu) vergleichen". 9 In dieser Sicht hat Sozialpolitik allenfalls noch am Rande Platz; einzig zugestanden wird ihr ein Einfluß auf die generelle Verteilung über das Steuersystem,

\footnotetext{
${ }^{6}$ H. Lampert (1991), S. 13

${ }^{7}$ ebd., S. 415

${ }^{8}$ N. Berthold (1990), S. 171

${ }^{9}$ R. Vaubel (1983), S. 151
} 
die freilich den davon ausgehenden negativen Anreizwirkungen Rechnung zu tragen hat. ${ }^{10}$

Aus dem so skizzierten Standpunkt resultiert im Umgang mit Themen aus dem Gebiet der Sozialpolitik üblicherweise die Frage nach dem Vorliegen von "Marktversagen" als notwendiger Bedingung für die Legitimierbarkeit sozialpolitischer Aktivitäten. Es wird sich zeigen, daß und mit welchen Folgen die Debatte um die Pflegesicherung in Deutschland, sofern sie von Ökonomen geführt wird, üblicherweise einem solchen Ansatz folgt. Negiert wird damit die Möglichkeit der Existenz von Grenzen, jenseits derer die marktwirtschaftliche Ordnung nicht bloß auf "technișche" Schwierigkeiten stößt, sondern kraft spezieller gesellschaftlicher Zielvorstellungen im Grundsatz nicht gelten soll. Im Gegensatz dazu berücksichtigt die vorliegende Arbeit die im folgenden $z u$ begründende These, daß die Existenz solcher vom Marktprozeß ausgenommener Felder im Bereich der Sozialpolitik nicht nur gedanklich "zulässig", sondern auch geeignet ist, real beobachtbare sozialpolitische Phänomene zu erklären.

Als Ausgangspunkt der Argumentation kann dabei die Beobachtung dienen, daß ein beträchtlicher Anteil sozialpolitischer Leistungen in modernen Wohlfahrtsstaaten nicht in monetärer, sondern in Sachform gewährt wird, obwohl diese aus Sicht der Empfänger gleichwertigen Geldleistungen unterlegen sind. Hieraus folgert WISEMAN, daß - zumindest in der Wahrnehmung der jeweiligen Regierungen - auf seiten der "Geber", also der Steuer- und Beitragszahler, eine gewisse Vorliebe für spezifische Sachtransfers besteht. ${ }^{11}$ Er erklärt dies mit einer Interdependenz von Nutzenfunktionen im altruistischen Sinn, die aber auf solche Güter beschränkt bleibt, die "Gefühle der Fürsorge (caring) erzeugen". ${ }^{12}$ Eine solche Interpretation wahrt - bei allen Schwierigkeiten, die sie für die ökonomische Analyse birgt - wenigstens formal den Rahmen der Wohlfahrtsökonomie, sofern die dort maßgebliche individuelle Nutzenfunktion für solche Interdependenzen prinzipiell offen ist.

THUROW hingegen hält dieses Argumentationsmuster nicht für überzeugend; innerhalb der Logik der herkömmlichen Nutzenfunktion könne ein unmittelbares Interesse am Konsum bestimmter Güter durch andere nur im Fall spezieller Externalitäten bestehen,

\footnotetext{
${ }^{10} \mathrm{vgl}$. ebd., S. 163

$"$ siehe J. Wiseman (1985), S. 99

12 ebd., S. 94
} 
die aber z.B. im Fall des sachleistungsintensiven Gesundheitswesens in der Regel ${ }^{13}$ auf der gesamtgesellschaftlichen Ebene gerade nicht vorlägen. ${ }^{14}$ Er geht daher in seiner Interpretation einen Schritt weiter und nimmt eine Unterscheidung zwischen "private-personal" und "individual-societal" preferences vor. Während erstere das Verhalten innerhalb der Spielregeln (eines Wirtschaftssystems) bestimmen, betreffen letztere die (gesellschaftlichen) Spielregeln selbst. Da beide Präferenztypen unterschiedliche Handlungsebenen betreffen, können sie gar nicht in Widerspruch zueinander geraten.

Es ist demnach einer marktwirtschaftlich organisierten und demgemäß von marktwirtschaftlichem Verhalten geprägten Gesellschaft anheimgestellt, im politischen Prozeß darüber zu befinden, welche Güter in einer Weise verteilt werden sollen, die vom Marktprozeß abweicht. Es wird sich dabei um Güter von herausragender Wichtigkeit handeln, deren Nutzung der Status von Grundrechten zuerkannt wird und die - in Relation zum jeweils herrschenden Wohlstandsniveau - so teuer sind oder sein können, daß eine Verteilung auf Basis ungleicher Einkommen existenzielle Folgen hätte. ${ }^{15}$ In Anwendung auf die Gesundheitsversorgung in Sachleistungsform stellt THUROW die These auf, "that a society's interest in the distribution of medical care springs (...) from our individual-societal preferences that 'the rights of man' include an equal 'right to life' itself". ${ }^{16}$ Zur Unterstützung seines Ansatzes verweist THUROW auf die demokratische Grundregel "One man, one vote", die in ihrem normativen Gehalt auch oder gerade in marktwirtschaftlich verfaßten Gesellschaften weithin unstrittig ist. Wäre der Marktmechanismus als gesellschaftliches Axiom unumschränkt, so müßte doch auch das "Gut" politische Teilhabe konsequenterweise nach Zahlungsbereitschaft und damit auf Basis der jeweils gegebenen Einkommensverteilung zugewiesen werden!

Auch bei AARON findet sich diese Querverbindung zwischen Wahlrecht und medizinischer Versorgung, die er als Fälle eines "commodity egalitarianism" kennzeichnet. Für die Gesundheitsversorgung spricht er diesem Phänomen zumindest für die

\footnotetext{
${ }^{13}$ Anders verhält es sich im Fall ansteckender Krankheiten.

${ }^{14}$ L.C. Thurow (1974), S. 191: "Death is the most private of all activities."

${ }^{15}$ Die letzte Bedingung ist in Industriestaaten z.B. für Grundnahrungsmittel nicht erfüllt. Der mühelose Zugang eines jeden zu einer hinreichenden Menge davon ist bereits über die Einkommensverteilung mehr oder weniger lückenlos gewährleistet. In armen Ländern ist das anders und z.B. eine massive Subventionierung des Brotpreises dementsprechend weit verbreitet.
}

${ }^{16}$ L.C. Thurow (1974), S. 192, Hervorhebung im Original 
Vergangenheit große empirische Relevanz zu, wenn er feststellt, daß "die meisten entwickelten westlichen Staaten im zwanzigsten Jahrhundert" die Entscheidung getroffen hätten, "die Gesundheitsversorgung außerhalb des Marktes anzusiedeln". ${ }^{17}$

Es findet also die Forderung nach Selbstbeschränkung der Ökonomie im Umgang mit Gegenständen der Sozialpolitik ihre Konkretion darin, daß die einkommensunabhängige Verteilung bestimmter Güter des "Grundbedarfs", also evtl. auch von Pflegeleistungen, als mögliches Ziel berücksichtigt werden soll. Keineswegs ist eine apodiktische und absolute Setzung dieses Ziels beabsichtigt. Vielmehr wird es neben den ökonomisch begründbaren Zielen auf seine tatsächliche Relevanz und seine Implikationen hin untersucht. Bejaht wird hier allein die Frage seiner Zulässigkeit im Rahmen einer ökonomischen Betrachtung.

Bevor auf andere Punkte eingegangen wird, an denen die Forderung nach Eigenständigkeit der Sozialpolitik gegenüber ökonomischen Denkmustern konkrete Folgen für die vorliegende Arbeit hat, sei der Hintergrund obiger Überlegungen zum Gleichheitsziel in bezug auf bestimmte Güter noch kurz angeleuchtet: Bemerkenswerterweise hat diese Denkrichtung, was den deutschsprachigen Raum betrifft, in der jüngeren sozialökonomischen Debatte kaum Niederschlag gefunden. ${ }^{18}$ In der angelsächsischen Literatur und hier insbesondere in Großbritannien ist das anders. ${ }^{19}$ Zwar entspricht die dort verbreitete Lehre in besonderem Maße der nach dem zweiten Weltkrieg betriebenen Politik (insbesondere der Errichtung des National Health Service $)^{20}$, jedoch reicht dies zur Erklärung insofern nicht aus, als auch die bundesdeutsche Sozialversicherung der Idee einer gleichmäßig und bedarfsgerechten medizinischen Versorgung verpflichtet ist. ${ }^{21}$

So ist es nicht nur wiederum ökonomistisch, sondern auch ein gedanklicher Kurzschluß, die Idee der Verteilung von Gesundheitsgütern allein nach Bedarf ohne weiteres mit einem "staatlichen Zwangsgesundheitssystem" gleichzusetzen, das "mit erheblichen ökonomischen Nachteilen verbunden" sei und daher "aus der weiteren Betrachtung

\footnotetext{
${ }^{17}$ H. Aaron (1981), S. $21 \mathrm{f}$.

${ }^{18}$ Als Ausnahme siehe etwa F. Hengsbach/M. Möhring-Hesse (1992), S. $120 \mathrm{f}$.

${ }^{19}$ vgl. F. Schulz-Nieswandt (1992), S. 244

${ }^{20}$ siehe R. M. Titmuss (1976), S. 129f.

${ }^{21}$ vgl. etwa J. Frerich (1990), S. 283
} 
ausgeschlossen werden" könne. ${ }^{22}$ Vielmehr ist auch ein Sozialversicherungssystem ohne staatliche Erzeugung der Gesundheitsleistungen selbst in der Lage, den Anspruch auf Einkommensunabhängigkeit des Leistungsgeschehens im Gesundheitsbereich einzulösen, zumal dann, wenn es auf die Gesamtbevölkerung ausgedehnt wird..$^{23}$

Gleichwohl ist PETERSENs kategorische Sicht durchaus typisch für die deutsche bzw. deutschsprachige sozial- und gesundheitsökonomische Szene, wie ein Blick in weitere grundlegende Lehrbücher zeigt: Auch BREYER/ZWEIFEL sind bemüht, den Grundbedarfs-Ansatz auf eineinhalb Druckseiten ad absurdum zu führen, um sich im übrigen ungestört einer durchweg auf Zahlungsbereitschaften basierenden Betrachtung zu widmen. ${ }^{24}$ In weniger streng ökonomisch ausgerichteten Werken ${ }^{25}$ ist der güterspezifische Gleichheitsgedanke überhaupt nicht auffindbar. ${ }^{26} \mathrm{Daß}$ einer solchen Ausblendung kritische Punkte zum Opfer fallen, deren sich "Gründungsväter" auch der deutschen Nationalökonomie noch bewußt waren, läßt sich für BÖHM-BAWERK zeigen, der die Abhängigkeit der am Markt relevanten Zahlungsbereitschaft von der höchst unterschiedlichen Zahlungsfähigkeit der Einzelnen anhand mehrerer Beispiele problematisiert und als "Nachteilige Wirkung des freien Wettbewerbs" einstuft; dies sogar ohne ausdrückliche Einschränkung auf Güter von existenzieller Qualität. ${ }^{27}$ Inzwischen aber scheint eine ökonomistische Verengung eingetreten zu sein, deren Aufhebung zum besseren Verständnis und zur konstruktiveren Behandlung sozialpolitischer Probleme dienlich sein kann.

Zur Vermeidung von Mißverständnissen sei betont, daß die Öffnung der ökonomischen Analyse für marktfremde Ziele keineswegs auf das Postulat hinausläuft, die Sozialpolitik

${ }^{22}$ so G. Petersen (1989), S. 40 und 42

${ }^{23}$ Diese Unabhängigkeit zwischen der Trägerschaft des Angebots und Gleichheitszielen betont auch A.J. Culyer (1991), S.69.

${ }^{24}$ siehe F. Breyer/P. Zweifel (1992), S. 151f: Ihre Argumente sind an dieser Stelle sämtlich schwach: $\mathrm{Da}$ ein bedarfsorientiertes System Rationierung nach sich zieht, ist keineswegs zwingend, sondern eine Frage der gesellschaftlichen Finanzierungsbereitschaft, die problematische Ab- und Eingrenzung von Grundbedarf ist politisch zu klären. Absurd ist schließlich die Behauptung, daß in der Konsequenz des Gleichheitsgedankens auch eine polizeistaatliche Unterbindung gesundheitsschädlicher Verhaltensweisen erfolgen müsse.

${ }^{25}$ etwa J. Frerich (1990) und H. Lampert (1991)

${ }^{26}$ In bezug auf Lampert kritisiert dies auch F. Schulz-Nieswandt (1992), S. 244.

${ }^{27}$ Vgl. E. Böhm-Bawerk (1924), S. 475ff. Den Hinweis verdanke ich Jürgen Volkert (1991), S. $99 \mathrm{ff}$. 
letztlich den Nichtökonomen zu überlassen. Eher im Gegenteil kann sie dazu dienen, die Nähe der Wirtschaftswissenschaft zu realpolitischen Problemen und damit ihre praktischen Möglichkeiten zur Einflußnahme zu erhöhen. So befürchtet WILLIAMS, gerade die Vernachlässigung außermarktlicher Aspekte "will exclude economists from exercising their analytical skills over large areas of social policy where the ethic of the market $(\ldots)$ is rejected" und erblickt darin "a great waste of our talents". ${ }^{28}$

Weiterhin ergibt sich aus den einleitenden Überlegungen zum Verhältnis zwischen Wirtschaftswissenschaft und Sozialpolitikdie Forderung nach einer möglichst differenzierten und präzisen Erfassung der in Betracht kommenden oder feststellbaren sozialpolitischen Zielkomponenten im einzelnen. Was damit gemeint ist, läßt sich mit Blick auf die Pflegedebatte in Deutschland wiederum am besten ex negatione erläutern. So ist beispielsweise mit Recht kritisiert worden, daß in dieser Diskussion "fast ausschließlich die Finanzierungsproblematik und die Bestimmung des Kostenträger erörtert worden [sei], während die pflegerische Versorgung (Leistungserbringung) selbst nur selten angesprochen worden ist". ${ }^{29}$ Dies ist sicherlich auch auf die Durchsetzungsfähigkeit von Ökonomen zurückzuführen, die die Debatte als eine vorwiegend finanzwissenschaftliche geprägt haben. Aber auch unterhalb dieser problematischen Akzentuierung der Pflegediskussion insgesamt wird im Verlauf der Arbeit deutlich werden, daß der "methodische Monetarismus", also die vorrangige Beachtung von Geldströmen bzw. Marktprozessen an vielen Punkten zu einer verkürzten Problemsicht geführt hat. ${ }^{30}$

Dabei ist es keineswegs so, daß sich solche oft vernachlässigten Aspekte einer ökonomischen Erfassung von vornherein entzögen. Allerdings muß sich eine solche Analyse so weit wie möglich auf die Besonderheit des Gegenstandes einlassen und sich dabei der Erkenntnisse sozialwissenschaftlicher Forschungszweige außerhalb der Ökonomie bedienen. Ein Anspruch auf wirkliche "Interdisziplinarität", wie sie LAMPERT fordert ${ }^{31}$, erwächst daraus für die vorliegende Arbeit freilich nicht; er wäre im gegebenen Rahmen nicht einzulösen. In den Augen des Verfassers würde

\footnotetext{
${ }^{28}$ A. Williams (1981), S. 281

${ }^{29}$ G. Igl (1986), S. 195

${ }^{30}$ Beispiele dafür sind die Vernachlässigung innerfamiliärer Kosten und Nutzen, des nichtmonetären Schadens der Pflegebedürftigkeit und der Frage der Qualität von Pflegeleistungen.
}

${ }^{31}$ H. Lampert (1985), S. 13: "Wissenschaftliche Sozialpolitik braucht interdisziplinäre Zusammenarbeit." 
es vollauf genügen, wenn die Untersuchung einen Beitrag zur Auflockerung der recht verbreiteten Frontstellung bzw. der wechselseitigen Ignoranz zwischen Ökonomie und Sozialpolitik leistete, indem sie für Angehörige beider "Parteien" von Interesse wäre.

\section{3 Überblick}

Der Aufbau der Arbeit gestaltet sich wie folgt: Zuerst wird im folgenden Kapitel der Pflegefall als Risiko beschrieben und charakterisiert und im Anschluß die Frage behandelt, warum eine freiwillige Absicherung gegen seine Folgen auch dann vielfach ausbleibt, wenn sie eine Verbesserung der Wohlfahrt verspricht. Wie in der gesamten Arbeit konzentriert sich die Betrachtung dabei auf den typischen Fall der Pflegebedürftigkeit im fortgeschrittenen Alter.

Kapitel 3 trägt dem sozialpolitischen Gehalt des Untersuchungsgegenstandes Rechnung, indem dort der Versuch unternommen wird, das Schutzziel inhaltlich auszufüllen. So entsteht ein Anforderungsprofil in bezug auf verschiedene Aspekte der zu gewährenden Leistungen. Am Schluß des Kapitels wird die oben erörterte Möglichkeit aufgegriffen, Pflegeleistungen als Güter des Grundbedarfs aufzufassen. Für diesen Fall ergibt sich in einigen Punkten eine veränderte Formulierung des Kriterienkatalogs.

Im vierten Kapitel wird untersucht, unter welchen Voraussetzungen ein den Anforderungen an die Schutzwirkung genügendes System auch als effizient gelten kann. $\mathrm{Zu}$ nächst kommt dabei die Finanzierung der Pflegesicherung ins Spiel. Deren Modus und die durch sie bewirkte Verteilung der Lasten sind von Bedeutung sowohl für die effizienznotwendige Verläßlichkeit der Schutzzusage als auch für die Frage, inwieweit die Einführung einer Pflegesicherung als Pareto-Verbesserung gewertet werden kann. Im zweiten Teil des Kapitels wendet sich die Untersuchung erneut der Leistungsseite zu. Dabei geht es insbesondere um das Problem von Verhaltensänderungen, die durch die Versicherung bewirkt und von denen Effizienzverluste ausgelöst werden können. Im Zentrum steht dabei nicht die allgemeine Theorie solcher Effekte, sondern die Möglichkeiten ihrer konkreten Ausprägung im Fall einer Pflegeversicherung.

In Kapitel 5 wird die gesetzliche Pflegeversicherung in den Niederlanden als der empirische Gegenstand der Untersuchung vorgestellt. Im Anschluß an einen Abriß der Entstehungsgeschichte und der Entwicklungschronologie des Systems erfolgen 
eine Darstellung des Regelwerks in seiner gegenwärtigen Form sowie die Aufbereitung wesentlicher Leistungs- und Kostendaten. Am Schluß des Kapitels steht der Versuch, den Beobachtungszeitraum anhand aktueller Entwicklungen auf die Zukunft hin zu erweitern. Hier kommt insbesondere die Reform des niederländischen Gesundheitsbzw. Krankenversicherungswesens zur Sprache, in deren Einzugsbereich auch die Pflegeversicherung in Teilen gehört.

Im sechsten Kapitel werden die Effektivitätsmaßstäbe gemäß Kapitel 3 und die Effizienzkriterien aus Kapitel 4 zur Anwendung gebracht, um zu einer möglichst differenzierten Bewertung der Pflegesicherung in den Niederlanden zu gelangen. In verkürzter Form wird schließlich in Kapitel 7 auch die künftige deutsche Pflegeversicherung einer solchen Prüfung unterzogen. Begrenzt ist deren Erörterung nicht nur durch den Zuschnitt der Arbeit, sondern auch deswegen, weil auf Erfahrungen mit dem Funktionieren der Versicherung in Deutschland noch nicht zurückgegriffen werden kann. 


\section{Der Pflegefall als ungedecktes Risiko}

\subsection{Das Risiko, pflegebedürftig zu werden: Eine Umschreibung}

Pflegebedürftigkeit sinnvoll und klar zu definieren, ist keine leichte Aufgabe. Möglicherweise ist es auch verfehlt, nach einer allgemeinen Begriffsbestimmung überhaupt zu suchen, wenn man bestrebt ist, bloß tautologische Umschreibungen zu vermeiden. So gilt nach dem BSHG als pflegebedürftig eine Person, die "so hilflos ist, daß sie nicht ohne Wartung und Pflege" bleiben kann ( $\$ 68)$. Mit dieser Abgrenzung ist zum einen nicht viel gewonnen, zum andern schließt sie offenkundig auch viele schwerere Fälle von Krankheit ein, die im vorliegenden Zusammenhang nicht von Interesse sind.

Damit ist bereits ein strittiger Punkt der Pflegedebatte berührt, nämlich die Frage, ob eine scharfe Grenzziehung zwischen Krankheits- und Pflegefall überhaupt möglich ist. Als Richtschnur einer Trennung zwischen beiden Phänomenen gilt dabei zunächst das Kriterium des Behandlungsbedarfs ${ }^{1}$; sein Vorliegen zeigt den Krankheitsfall an. Zweifelhaft aber ist, in welchen Fällen Pflegebedürftigen ein solcher Bedarf abzusprechen ist. Während BRANDT dazu die Einschätzung vertritt, der reine Pflegefall in diesem Sinne sei "eher die Ausnahme"2, gelangt ein älteres Gutachten des Kuratoriums Deutsche Altershilfe zu dem radikaleren Standpunkt, daß es solche Fälle angesichts des Fortschritts der Medizin überhaupt "nach ärztlichem Gewissen nicht mehr geben" dürfe. ${ }^{3}$ Insbesondere könne aus dem Fehlen einer Heilungschance keineswegs auf den Wegfall eines Behandlungsbedarfs geschlossen werden, weil sinnvolle Therapie auch darin bestehen könne, einen "Status quo zu erhalten (...) oder eine gewisse Linderung subjektiven Leidens zu ermöglichen". ${ }^{4}$ Daher seien "alle pflegerischen Maßnahmen (...) Bestandteil der Behandlung" und eine grundsätzliche Trennung von Krankheit und Pflegebedürftigkeit nicht zu rechtfertigen. Die Versorgung in Pflegeheimen, so wird gefolgert, falle in die Leistungspflicht der gesetzlichen Krankenkassen.

\footnotetext{
${ }^{1}$ vgl. Hans Brandt (1987), S. 119f.- Hinweis: Die Zählung der Fußnoten erfolgt kapitelweise.

${ }^{2}$ ebd., S. 120

${ }^{3}$ Kuratorium Deutsche Altershilfe (1979), S.8

${ }^{4}$ ebd., S. 9
} 
Wären die Rechtsprechung bzw. der Gesetzgeber dieser Auffassung gefolgt, so hätte sich für die Bundesrepublik die Frage der Pflegesicherung weitgehend erübrigt. Sie haben es nicht getan. Gleichwohl ist der angeführten Argumentation im Grundsatz schwerlich zu widersprechen. Auch die für den Vierten Familienbericht der Bundesregierung verantwortliche Sachverständigenkommission stellt fest, der Arzt sei " bei ganzheitlicher Betrachtung des chronisch kranken alten Menschen (...) kaum in der Lage, zwischen Pflegefall und Nichtpflegefall zu unterscheiden". ${ }^{5}$ Für letzteren aber gelte, daß "rein rechtlich gesehen die Behandlung eines kranken Menschen kostenmäßig eine Aufgabe der Krankenkassen" darstelle. ${ }^{6}$

Es liegt daher nahe, in einem ersten Schritt die Pflegebedürftigkeit negativ als das Vorliegen eines Betreuungsbedarfs zu definieren, der seiner medizinischen Bedingtheit zum Trotz nicht zum Leistungsbereich von Krankenversicherungen gehört. Dies wiederum ist in der Regel dann der Fall, wenn die Befriedigung des fraglichen Bedarfs außerhalb von Kliniken und damit nicht in ständiger Begleitung durch Ärzte vorgenommen wird. Es ist mithin ein überwiegend institutionelles Merkmal, das zur Trennung des Pflegefalls von der Krankheit herangezogen wird.

Aus den Schwierigkeiten bei der Trennung von Krankheit und Pflegebedürftigkeit als Ereignissen ergibt sich, daß auch die Risiken ihres Eintritts eng miteinander verwandt sind. Gemeinsam ist beiden insbesondere die Zufälligkeit und Unabsehbarkeit des Schadensfalles hinsichtlich der Fragen, ob, wann, in welcher Form und Schwere und für wie lange er eintritt. Ferner sind beide Fälle nicht nur mit finanziellen Kosten unterschiedlicher, mitunter "katastrophaler" Höhe verbunden, sondern auch mit "intangiblen" Nachteilen etwa in Form von körperlichem und physischem Leid und/oder einer Einschränkung von Entfaltungsmöglichkeiten. Sicherungsmechanismen richten sich demgemäß prinzipiell zum einen auf die Vermeidung der monetären, zum anderen auf die Verringerung der übrigen Schadenskomponenten, die im allgemeinen nicht restlos ausgeschaltet werden können. Unterschiede aus der Perspektive derjenigen, die sich mit den Risiken konfrontiert sehen, lassen sich nur in einer typisierenden, also nicht in allen Einzelfällen zutreffenden Sicht feststellen:

\footnotetext{
${ }^{5}$ Bundesminister für Jugend, Familie, Frauen und Gesundheit (1986), S. 153

${ }^{6}$ Es ist merkwürdig, daß die Kommission aus dieser Überlegung keine Forderungen ableitet, sondern lapidar konstatiert: "Bedingt durch die versicherungsrechtliche Lage wird dennoch die Unterscheidung gemacht" (ebd.).
} 
- Der Pflegefall tritt in der Regel pro Person nur einmal auf und endet oftmals erst mit dem Tod. Krankheiten sind hingegen meist vorübergehender Natur und treten wiederholt auf.

- Während nahezu jeder irgendwann einmal krank und behandlungsbedürftig wird, bleibt ein vergleichsweise hoher Prozentsatz der Menschen von Pflegebedürftigkeit verschont. ${ }^{7}$

- $\quad$ Pflegebedürftigkeit ist in weit höherem Maß mit dem Lebensalter korreliert als Krankheit. So haben in der Bundesrepublik nur 0,5\% der unter 65jährigen regelmäßigen Pflegebedarf, bei den $80-85$ jährigen aber bereits über 10 , bei den noch Älteren sogar mehr als $25 \% .{ }^{8}$ Die Bewohner von Pflegeheimen sind dabei noch nicht mitgezählt.

- Individuelles Vorbeuge-Verhalten hat auf das Krankheitsrisiko größeren Einfluß als auf das Pflegerisiko. Dies ergibt sich daraus, daß gesundheitsbewußtes Verhalten die Lebenserwartung erhöht. Das Erreichen eines hohen Alters wirkt aber an sich als wesentlicher Risikofaktor hinsichtlich der Pflegebedürftigkeit.

- $\quad$ Die dem Pflegefall entsprechenden Hilfsleistungen sind in höherem Maße als krankheitsbezogene Leistungen auch von Laien prinzipiell zu erbringen. Andererseits sind sie definitionsgemäß nicht durch individuelle Selbsthilfe substituierbar, was wiederum in leichteren Fällen von Krankheit der Fall sein kann. Es folgt aus diesen Merkmalen eine zentrale Rolle des privaten Umfelds im Pflegefall. Wenn etwa Angehörige bereitstehen, die willens und in der Lage sind, die Pflege unbezahlt und bedarfsgerecht selbst zu übernehmen, so entfällt jeder Sicherungsbedarf. ${ }^{9}$ In diesem Sinn läßt sich die Pflegebedürftigkeit als partiell "soziales Risiko" kennzeichnen, während der Krankheitsfall idealtypischerweise rein medizinisch bedingt ist.

- Während die Kosten der Pflege in der Summe weitaus niedriger sind als die des Gesundheitswesens im herkömmlichen Sinn ${ }^{10}$, ist der einzelne Pflegefall im Durchschnitt deutlich kostspieliger als eine Krankheit. Dies erklärt sich aus den bereits erwähnten großen Unterschieden hinsichtlich der Anzahl der Betroffenen und der Fallzahl pro Kopf. In der Bundesrepublik entfielen im

\footnotetext{
${ }^{7}$ Wohlgemerkt nur im hier angestellten Vergleich (siehe unten).

${ }^{8}$ siehe Infratest Sozialforschung (1992), S. 26

${ }^{9}$ W. Frank (1987), S. 228, bezeichnet daher die "Fremdpflegebedürftigkeit" als das eigentliche Risiko.

${ }^{10}$ In den Niederlanden z.B. betrug 1990 der Anteil aller Pflegekosten an den Aufwendungen für das Gesundheitssystem insgesamt weniger als ein Sechstel (errechnet nach Angaben bei Tweede Kamer (1992), S. 13 und 41).
} 
Jahre 1989 allein im Bereich der ambulanten ärztlichen Behandlung 5,23 Behandlungsfälle auf jeden Versicherten. ${ }^{11}$ Auch wenn oft mehrere Fälle im Sinne dieser Zählung ein und derselben Erkrankung zuzurechnen sein dürften, bleibt der Wert ungleich höher als der der "Pflegefälle pro Kopf".

In Zusammenfassung der genannten Punkte läßt sich das Risiko, pflegebedürftig zu werden, in folgender Weise umschreiben: Es handelt sich um ein Risiko, das hinsichtlich seines vor allem medizinisch bestimmten Inhalts, der Unabsehbarkeit seines Eintritts und seines Schadenscharakters dem Krankheitsrisiko benachbart ist. In Abgrenzung von diesem ist es gekennzeichnet durch die geringere Häufigkeit, die größere Dauerhaftigkeit und Unbeeinflußbarkeit sowie die hohen durchschnittlichen Kosten des Schadensfalls, dessen konkretes Ausmaß zudem von der familiären bzw. sozialen Lage des Betroffenen entscheidend mitbestimmt wird.

Auf den ersten Blick legen die meisten der angeführten Eigenschaften die Vermutung nahe, daß der Schutz gegen die Folgen der Pflegebedürftigkeit ein Versicherungsgegenstand par excellence sein müßte. Je unabsehbarer und - im finanziellen Ausmaß katastrophaler ein mögliches Ereignis, desto größer der Nutzen einer Versicherung für risikoaverse Subjekte. Die gezielte individuelle Bildung von Reserven ist in solchen Fällen keine sinnvolle Alternative, da sie entweder im nötigen Umfang gar nicht möglich oder aber ineffizient ist: Im recht wahrscheinlichen Fall des Nichteintritts der Pflegebedürftigkeit erweist sich der mit der Bildung einer Pflegerücklage verbundene erhebliche Konsumverzicht im Nachhinein als unnötig. ${ }^{12}$

Andererseits ist das Risiko keineswegs so klein, daß es vernünftigerweise vernachlässigt oder übersehen werden könnte: Einer Untersuchung für die Stadt Mannheim zufolge "lebte jeder zehnte der 1982 (...) verstorbenen Männer von 65 und mehr Jahren vor seinem Tod in einem Heim, von den Frauen sogar jede vierte". ${ }^{13}$ Unter Einschluß nichtstationärer Pflege, die ebenfalls mit erheblichen Kosten verbunden sein kann,

\footnotetext{
${ }^{11}$ Bundesminister für Arbeit und Sozialordnung (1990), S. 14

${ }^{12}$ Damit erhöht gerade auch die - im Vergleich zum (minder schweren) Krankheitsfall gemessene Seltenheit des Pflegefalls den potentiellen Nutzen einer Versicherung (vgl. G. Wagner (1991), S. 42). ${ }^{13}$ W. Rückert (1987), S. 79.
} 
müßte der Anteil derjenigen, die eine Pflegeversicherung gegebenenfalls irgendwann einmal in Anspruch nähmen, noch deutlich höher sein. ${ }^{14}$

Dennoch ist das Fehlen einer Pflegeversicherung, sei sie privat oder gesetzlich, in den meisten Industriestaaten für die große Mehrheit der Bevölkerung der Normalfall. Für beide Versicherungstypen gilt es im folgenden zu untersuchen, wie dieser Befund erklärt werden kann.

\subsection{Gründe für die geringe Verbreitung privater Pflegeversicherungen}

Anfang der 90er Jahre gaben in der Bundesrepublik nur $6 \%$ der Teilnehmer einer repräsentativen Umfrage an, eine private Pflegeversicherung abschließen zu wollen. ${ }^{15}$ $\mathrm{Daß}$ ein "Attentismus" angesichts der möglichen Einführung der Pflegesozialversicherung für dieses Resultat nicht entscheidend ist, läßt ein Blick in die USA vermuten: Dort hatten 1987 weniger als 0,25\% der Bevölkerung eine entsprechende Police erworben ${ }^{16}$, ohne daß sich eine gesetzliche Regelung abzeichnete. Dort wie in der Bundesrepublik steht diese Zurückhaltung in krassem Gegensatz zur Nachfrage nach privater Krankenversicherung, wo in der Regel ein möglichst umfassender Schutz angestrebt und nach Möglichkeit selbst zu hohen Prämien erworben wird. ${ }^{17}$ Gründe für die geringe Bedeutung privater Versicherungen liegen erstens auf der Angebotsseite, zweitens als subjektive Barrieren bei den potentiellen Nachfragern und drittens im institutionellen Umfeld, genauer: in der sozialen Mindestsicherung, wie sie ohne spezifischen Bezug zur Pflegeproblematik in modernen Industriestaaten üblicherweise gewährt wird.

\subsubsection{Angebotsseitige Gründe}

Verglichen mit anderen Versicherungssparten haben Policen für den Pflegefall erst seit kurzer Zeit überhaupt Eingang in das Sortiment von Versicherungsgesellschaften

\footnotetext{
${ }^{14}$ Pflegequoten pro Altersgruppe sind übrigens ungeeignet, um das individuelle Risiko abzubilden. Wenn man etwa feststellt, daß zu einem Zeitpunkt 6,7\% der über 64jährigen als "Personen mit regelmäßigem Pflegebedarf" einzustufen sind (Infratest (1992), S. 26), droht übersehen zu werden, daß einem großen Teil der übrigen 93,3\% der Eintritt in die Pflegebedürftigkeit jeweils noch bevorsteht.

${ }^{15}$ Stiftung Warentest (1991), S. 122

${ }^{16}$ siehe A.M. Rivlin/J.M. Wiener (1988), S. 59

17 vgl. ebenda
} 
gefunden. Mit der von WASEM für die Bundesrepublik konstatierten "defensiven Haltung zahlreicher Unternehmen bei der Etablierung des heutigen Angebotes"18 korrespondiert etwa in den USA die Beobachtung, daß zwar zahlreiche Angebote bestehen, deren Schutzwirkung aber unzureichend ist:"(...) insurers have built in features that minimize their financial risks, at the cost of reducing the financial protection given consumers. "19 In den Niederlanden ist das Pflegerisiko vor Einführung der Sozialversicherung als unversicherbar im marktwirtschaftlichen Sinn angesehen worden, weil private Angebote vollständig fehlten. ${ }^{20}$

Es kann also nach gegenwärtigem Sachstand festgehalten werden, daß der Abschluß von Pflegeversicherungen unbeschadet aller noch aufzuführenden Hemmnisse auf seiten der Nachfrage auch daran scheitern kann, daß dem Sicherungsbedürfnis angemessene Verträge nicht angeboten werden. Das liegt offenbar hauptsächlich daran, daß mit der Eintrittswahrscheinlichkeit des Pflegefalls einerseits und der durchschnittlich zu erwartenden Schadenshöhe andererseits die beiden für eine solide Prämienkalkulation benötigten Größen schon mittelfristig kaum verläßlich prognostizierbar sind ${ }^{21}$. Es versteht sich, daß die lange Zeitspanne, die typischerweise zwischen Vertragsabschluß und Versicherungsfall liegt, die Unsicherheit verstärkt. ${ }^{22}$

Was den erstgenannten Faktor, die Pflegewahrscheinlichkeit, betrifft, so ergibt sich die Entwicklungsunsicherheit aus der unter Medizinern strittigen Frage, ob "der medizinische Fortschritt eher Pflegebedürftigkeit vermeiden helfen oder (..) eine weitere Verbesserung der Lebenserwartung durch ein erhöhtes Risiko pflegebedürftig zu werden, erkauft" wird. ${ }^{23}$ Über die durchschnittliche Dauer der Pflegebedürftigkeit beeinflußt die Entwicklung in diesem Punkt auch die Kosten pro Fall, die zudem von der Preisentwicklung im Pflegesektor abhängt. Diese wiederum ist außer von der

\footnotetext{
18 J. Wasem (1991), S. 386

${ }^{19}$ A.M. Rivlin/ J.M. Wiener (1988), S. 65

${ }^{20}$ vgl. G.M.J. Veldkamp u.a. (1966), S. 1

${ }^{21}$ Aus den nachfolgend aufgeführten Unsicherheitsfaktoren folgt freilich nicht zwingend, daß eine umfassende Versicherung nicht angeboten werden könnte. Die Grenze der "Schätzbarkeit" eines Risikos als Voraussetzung seiner Versicherbarkeit ist nicht klar bestimmbar. Vgl. W. Karten (1993), S. 12f.

22 vgl. D. Geitner (1985), S. 32

${ }^{23}$ J. Wasem (1991), S. 379
} 
allgemeinen Inflationsrate insbesondere durch die Entwicklung der Personalschlüssel und die sektorspezifischen Lohnsteigerungen determiniert.

In der Bundesrepublik gilt das Altenpflegepersonal derzeit als überlastet und unterbezahlt. Anders wäre auch kaum zu erklären, daß der Heimbereich trotz hoher gesamtwirtschaftlicher Erwerbslosenzahlen zu Beginn der 90er Jahre mit erheblichen Nachwuchssorgen kämpft, denen man zum Beispiel durch massive Werbekampagnen zu begegnen versucht. Offensichtlich besteht hier also ein Nachholbedarf in Richtung auf die Aufstockung von Stellenplänen und/oder Gehältern, der für die Entwicklung der Pflegekosten große Risiken birgt. Überdies wirkt sich auch auf die erforderliche Personalstärke wiederum der medizinische Faktor aus, sofern Fortschritte bei der Lebenserhaltung die durchschnittliche Schwere stationär betreuter Pflegefälleerhöhen.

Es ist in Anbetracht der genannten Unsicherheiten auf Makroniveau nicht verwunderlich, wenn Versicherer, sofern sie überhaupt zu einem Engagement auf dem Pflegemarkt bereit sind, Vorkehrungen treffen, die ihr eigenes Risiko zumindest in bezug auf die Entwicklung der Kosten pro Leistungseinheit in Grenzen halten. ${ }^{24}$ So bemängelt etwa $\mathrm{BOHN}$, der im übrigen eine privatwirtschaftliche Lösung des Pflegeproblems verficht, die Existenz von Höchstsätzen pro Tag oder Jahr in allen am deutschen Markt angebotenen Policen und stellt in bezug auf diese fest: "Da sie (..) nicht dynamisiert sind, kann mit dieser Form der Pflegekostenversicherung die Werterhaltung des einmal abgeschlossenen Versicherungsschutzes nicht garantiert werden." ${ }^{25}$ Seine 1989 gemachte Vorhersage, derartige Leistungsgrenzen würden "auf Dauer (...) sicherlich gänzlich fallen"26, hat sich bislang nicht erfüllt: Die Angebote vom Typ der Pflegekostenversicherung, die prinzipiell einen festen und hohen Prozentsatz der Gesamtkosten zu übernehmen versprechen, arbeiten fast durchweg mit einer jährlichen Leistungsobergrenze von günstigstenfalls DM 60.000. ${ }^{27}$ Ein solcher Betrag wirkt bei heutigem Kostenniveau weitgehend unbedenklich, dürfte sich ohne Dynamisierung aber im Lauf der Zeit als entscheidender Engpaßfaktor erweisen. Bei anderen Versicherungstypen wie der Pflegetagegeldversicherung, deren

\footnotetext{
${ }^{24}$ W. Kaske (1985), S. 36 spricht in diesem Zusammenhang von "durchaus unterschiedlichen Auffassungen der einzelnen Unternehmen hinsichtlich der Versicherbarkeit dieses Risikos".

${ }^{25}$ K. Bohn (1989), S. 76

${ }^{26}$ ebenda

${ }^{27}$ Siehe im einzelnen Stiftung Warentest (1991), S. 123. Bei der einzigen Gesellschaft, die auf die feste Obergrenze verzichtet, beschränkt sich die Erstattung auf die Hälfte des Pflegesatzes.
} 
Leistungen nicht direkt an die tatsächlichen Kosten gekoppelt sind, ist die Bedarfsgerechtigkeit der Sätze ohnehin zweifelhaft.

Um einer "adverse selection", also der überproportionalen Wahrnehmung ihrer Angebote durch schlechte Risiken vorzubeugen, verlangen die Versicherer zumindest bei höherem Eintrittsalter und/oder hohen Leistungszusagen vor Vertragsabschluß eine Gesundheitsprüfung. Oberhalb einer Altersgrenze von 55 bis 65 Jahren wird ein Vertragsabschluß generell abgelehnt. ${ }^{28}$

Schließlich ist - ohne daß hier ein Nachweis geführt werden könnte - anzunehmen, daß die genannten Unsicherheiten über die Entwicklung des Schadensvolumens bei den überhaupt angebotenen Policen zu recht hohen Prämien führen, da die Versicherer zur Eingrenzung ihres eigenen Risikos ihren Kalkulationen ein eher ungünstiges Szenario zugrundelegen. $\mathrm{Zu}$ einem Hindernis für den Vertragsabschluß wird dies freilich nur dann, wenn die potentiellen Versicherungskunden ihre Zahlungsbereitschaft nicht auf ähnlich pessimistische Annahmen stützen. Dies ist im folgenden Abschnitt zu prüfen.

\subsubsection{Subjektive Gründe auf seiten der Nachfrage}

Ein naheliegendes Erklärungsmuster ist die in Zusammenhang mit meritorischen Gütern oft behauptete "Minderschätzung zukünftiger Bedürfnisse". Ein solches Phänomen könnte für den Schutz vor den Folgen des Pflegefalls deshalb von Bedeutung sein, weil dieser in der Regel erst in späten Lebensphasen eintritt. Die große Beliebtheit von Lebensversicherungen spricht allerdings dagegen, daß eine solche Haltung stark verbreitet ist. ${ }^{29}$ Plausibler erscheint die These, daß die Möglichkeit, pflegebedürftig zu werden, als besonders unerfreuliche Aussicht in hohem Maße der Verdrängung anheimfällt. Der bereits erwähnten Umfrage zufolge "hat weniger als die Hälfte [der Befragten, S.W.] hin und wieder die Befürchtung, sie könnte im Alter zum Pflegefall

\footnotetext{
${ }^{28}$ In den USA können bemerkenswerterweise auch bis zu 80jährige noch eine Pflegeversicherung abschließen. Die Prämien sind dann allerdings mehr als doppelt so hoch wie für 65jährige (siehe A.M. Rivlin/ J.M. Wiener (1988), S. 60f.).

${ }^{29}$ vgl. W. Buchholz/ W. Wiegard (1992), S. 451
} 
werden. ${ }^{130}$ Entscheidend ist dabei wohl der bereits genannte Tatbestand, daß der Kern des Übels (Autonomieverlust, stark eingeschränkte Lebensmöglichkeiten) durch eine Versicherung nicht berührt wird. ${ }^{31}$

Letzteres könnte auch ohne vollständige Verdrängung des Pflegerisikos eine Rolle spielen, wenn die Unzuträglichkeit des Pflegefalls als solchen den Nutzen der Versicherungsleistung a priori gering oder zweifelhaft erscheinen läßt. Ein solcher Nutzen kann in der Gewährung "besserer" Pflege, im Schutz des Vermögens und/oder darin bestehen, daß eine Abhängigkeit von Fürsorgeleistungen im Sinne der Sozialhilfe vermieden wird. Wenn die drohende Pflegebedürftigkeit an sich als größtmögliches Unglück eingestuft wird, mögen solche Vorteile als unbedeutend erscheinen. Oder auch als unsicher, sofern angenommen werden muß, daß sich Wahrnehmung und Präferenzen mit Eintritt des Pflegefalls verändern.

Unterstellt man, daß die genannten Vorteile der Versicherung im Pflegefall von den bereits Betroffenen im allgemeinen sehr wohl als solche wahrgenommen und geschätzt werden, so liegt in der Tat eine Fehleinschätzung eigener Bedürfnisse vor, die aber nicht aus deren Zukünftigkeit allein resultiert, sondern aus ihrem situationsspezifischen Charakter, dessen Verständnis beim vorerst nicht Pflegebedürftigen ein ausgeprägtes Vorstellungsvermögen voraussetzt. Ein anderer Aspekt, der sich real mit dem hier angesprochenen vermischen kann, ist die objektive Unsicherheit über das Ausmaß, in dem die Versicherungsleistungen die genannten Schutzzwecke erreichen werden, inwiefern also adäquate Pflege und dauerhafter finanzieller Schutz wirklich durch den Versicherungsabschluß gewährleistet sind. Dies verweist auf die im vorigen Abschnitt besprochenen Angebotsmängel zurück, die derartige Zweifel vielfach begründet erscheinen lassen.

\footnotetext{
${ }^{30}$ Stiftung Warentest (1991), S. 122. Siehe auch wiederum A.M. Rivlin/J.M. Wiener (1988), S. 64: "Several other surveys show that the elderly do not believe they will need long-term care (...). People seem to accept the possibility that they will someday get sick and visit a doctor or enter a hospital, but few seem willing to accept the risk of their becoming seriously disabled and using expensive home care or nursing home services."

${ }^{31}$ Zwar ließe sich für die Risiko-Lebensversicherung ähnlich argumentieren. Da aber deren Leistung im Versicherungsfall direkt den Hinterbliebenen zugute kommt, spielt das Verantwortungsgefühl gegenüber diesen Begünstigten bzw. auch deren Mitsprache hier wohl eine entscheidendere Rolle. Buchholz/Wiegard (1992), S. 451, weisen zudem auf die Rolle solcher Policen bei der Kreditabsicherung hin.
} 
Ein weiteres Hemmnis bei der Entscheidung für eine Pflegeversicherung stellt die Zuversicht dar, im Ernstfall von Angehörigen gepflegt zu werden. ${ }^{32}$ In der erwähnten Befragung äußerten fast $70 \%$ der Stichprobe eine solche Erwartung, die sich in vielen Fällen als irrig herausstellen wird. Daß eine große Mehrheit sich auf diese Option verläßt, zeugt eindeutig von einer verharmlosenden Sicht der Problematik, da selbst bei zweifelsfreier Opferbereitschaft der Angehörigen unsicher ist, ob diese im evtl. erst Jahrzehnte später eintretenden Pflegefall noch zur Verfügung stehen. Überdies sind selbst die motiviertesten und leistungsfähigsten Familienmitglieder in schweren Pflegefällen regelmäßig überfordert und zumindest auf professionelle Unterstützung angewiesen.

Offensichtlich neigen also die Befragten im letztgenannten Punkt zum Wunschdenken, da sie es vorziehen, durch Angehörige gepflegt zu werden. ${ }^{33}$ Einer solchen Präferenz aber läuft es tendenziell zuwider, sich für den Erhalt professioneller Pflegeleistungen zu versichern. Vielmehr wird gerade durch das Fehlen einer Versicherung möglicherweise ein erwünschter Anreiz für die Angehörigen geschaffen, die Pflege selbst $\mathrm{zu}$ übernehmen, um nicht für die Kosten professioneller Betreuung einstehen zu müssen. ${ }^{34} \mathrm{Ob}$ eine solche Taktik in sich rational sein kann, sei vorerst dahingestellt. ${ }^{35}$ Allemal bedenklich ist daran die Illusion, daß die bevorzugte Pflegeform in allen Fällen möglich sei. Es wird also in Wirklichkeit nur ein Teil des Risikos auf die Angehörigen abgewälzt, der Rest bleibt ungedeckt.

Nicht zuletzt sind es die hohen Prämien, die vielfach den Abschluß einer Versicherung verhindern. Dies gilt insbesondere für diejenigen, die sich aus den oben genannten Gründen erst in höherem Alter des Pflegerisikos bewußt werden. Nun ist die Prämienhöhe freilich idealtypisch zunächst einmal eine Folge der (etwa im Vergleich zu einer Feuerversicherung) recht hohen Wahrscheinlichkeit, mit der recht hohe Leistungen in Anspruch genommen werden. Als Hinderungsgrund kann sie bei

\footnotetext{
${ }^{32}$ vgl. A. Prinz (1987), S. 41

${ }^{33}$ Im Familienbericht der Bundesregierung wird eine Befragung erwähnt, derzufolge dies für $93 \%$ der Pflegebedürftigen gilt (siehe Bundesminister für Jugend, Familie, Frauen und Gesundheit (1986),

S. 175).

34 siehe zu diesem Gedanken M.V. Pauly (1990), S. $164 \mathrm{f}$.

${ }^{35}$ Das Leitmotiv ist dabei die Vorstellung einer drohenden Heimeinweisung als einziger Alternative zur vollständigen Übernahme der Pflege durch Angehörige. Es wird verkannt, daß sich private und bezahlte Pflege in der häuslichen Umgebung auch zum allseitigen Nutzen ergänzen können.
} 
Rationalverhalten nur dann dienen, wenn entweder eine Budgetschranke vorliegt, die die Prämie unerschwinglich macht, oder aber in dem Maße, wie die Prämie das "aktuarisch faire" Niveau, das sich allein aus der Wahrscheinlichkeit und der Höhe des Schadens ergibt, übersteigt. Der erste Aspekt spielt gewiß eine beachtliche Rolle: Im Jahre 1991 betrug die für ein angemessenes Leistungsniveau erforderliche monatliche Prämie für Frauen bei einem Eintrittsalter von 55 Jahren in Deutschland je nach Anbieter zwischen DM 108 und $128 .{ }^{36}$ Eine solche Belastung dürfte insbesondere nach dem Ausscheiden aus dem Erwerbsleben für viele Frauen untragbar sein. Für die USA konstatieren RIVLIN/WIENER: "Premiums are too expensive for most elderly. ${ }^{37}$

Der zweite Aspekt ist im vorigen Abschnitt bereits berührt worden, indem festgestellt wurde, daß die "wahre" Pflegewahrscheinlichkeit ebenso wie die erwartbare Schadenshöhe nur geschätzt werden kann und die Versicherer sich evtl. eher ungünstiger Prognosen bedienen werden. ${ }^{38}$ Seitens der Versicherten hingegen kann das beschriebene Wunschdenken zu einer Unterschätzung des Risikos und damit der "angemessenen" Prämie führen. Ein anderer, besser faßbarer und allgemeiner gültiger Grund für ein Mißverhältnis von Leistung und Gegenleistung in der Pflegeversicherung kommt im folgenden gesondert zur Sprache.

\subsubsection{Die garantierte Mindestsicherung als Grund für die Nachfrage- schwäche}

Eine wichtige Ursache für die geringe beobachtbare Nachfrage nach privaten Pflegeversicherungsverträgen ist darin zu sehen, daß in wirklichen finanziellen Notlagen, wie sie auch durch Pflegebedürftigkeit verursacht werden können, stets auf eine staatliche Mindestsicherung, im Falle Deutschlands die Sozialhilfe, zurückgegriffen werden kann. Im Extremfall eines Individuums, das keinerlei Vermögen und auch

\footnotetext{
${ }^{36}$ Stiftung Warentest (1991), S. 123

${ }^{37}$ A.M. Rivlin, J.M. Wiener (1988), S. 60

${ }^{38}$ Es versteht sich zudem, daß Anbieter von Pflegeversichenungen in der Öffentlichkeit zu Werbezwecken mit ungünstigen Prognosen arbeiten werden, die aufgrund der wirklichen Ungewißheit nicht widerlegbar sind. Da die Nach frager dieses Übertreibungsinteresse durchschauen, werden sie nicht sehr empfänglich für "Aufklärungskampagnen" seitens der Anbieter sein. Auf ebensolche Versuche aber setzen Buchholz/Wiegard (1992), S. 450, um die Möglichkeit "einer systematischen Unterschätzung des Pflegerisikos" im weiter oben diskutierten Sinn von der Hand zu weisen.
} 
kein Einkommen oberhalb des Existenzminimums zu verlieren hat, ist eine solche Vorrichtung einer Versicherung äquivalent, ein Anreiz zum Abschluß der letzteren also nicht gegeben. Das gilt selbstverständlich auch dann, wenn ein Mehreinkommen zwar vorhanden, aber so gering ist, daß es die Höhe der zu zahlenden Prämie nicht erreicht.

In einer Modellanalyse ${ }^{39}$ weisen BUCHHOLZ und WIEGARD nach, daß zwischen diesem Niveau und einem Einkommen, das hinreichend hoch ist, um den Rückgriff auf Sozialhilfe auch für den Pflegefall auszuschließen, unter üblichen Annahmen ein kritisches Einkommen existiert, bis zu dem der Abschluß einer Versicherung aufgrund der Sozialhilfegarantie trotz Risikoscheu unattraktiv wird. Entscheidend für diesen Effekt ist die Tatsache, daß die Sozialhilfeleistung nur bei Bedarf gewährt wird, also nicht mit Versicherungsleistungen kumuliert werden kann. Dies hat für Geringverdiener zur Folge, daß eine nach der Gesamtleistung kalkulierte Versicherungsprämie gezahlt werden müßte, während der damit erzielte Vorteil im Pflegefall sich auf die Differenz zwischen dem dann verfügbaren eigenen Einkommen und dem Existenzminimum beschränkt. Oder anders formuliert: Ein "aktuarisch faires" Verhältnis zwischen Prämie und Leistungszusage wird dadurch unmöglich, daß der Versicherungsnehmer neben der Prämie auch mit dem Wegfall eventueller Sozialhilfeleistungen "bezahlt". Je höher diese Leistungen, je höher der Durchschnittsschaden oder die Eintrittswahrscheinlichkeit (und damit die Prämie), desto höher liegt auch die kritische Einkommensgrenze, desto mehr Menschen werden also durch die Sozialhilfe vom Versicherungskauf abgehalten. ${ }^{40}$ Unter Wohlfahrtsaspekten ist diese Situation unbefriedigend.

Wie gewichtig dieser Punkt im Verhältnis zu den zuvor aufgeführten wirklich ist, läßt sich schwer entscheiden. Jedenfalls ist auf einige relevante Aspekte des Sozialhilfebezugs hinzuweisen, die in der oben referierten Modellbetrachtung ausgeblendet bleiben und deren Berücksichtigung den beschriebenen Effekt abschwächt:

- Bevor Sozialhilfe bezogen werden kann, muß nicht allein das Einkommen, sondern auch jegliches Vermögen eingesetzt und verbraucht werden. ${ }^{41}$ Wenn

\footnotetext{
${ }^{39}$ W. Buchholz/W. Wiegard (1992)

${ }^{40}$ siehe im einzelnen ebenda, S. 447

41 "Die Sozialhilfe wird (...) erst dann tätig, wenn alle Möglichkeiten der Selbsthilfe (...) ausgeschöpft (...) sind." J. Frerich (1990), S. 204
} 
also Vermögen - etwa in Form von Immobilienbesitz - auch bei Beziehern geringer (Alters-)Einkommen anzutreffen ist, verändert ein Interesse an dessen Bewahrung das Kalkül; der Abschluß einer Versicherung wird attraktiver.

- Zumindest im deutschen Sozialhilfesystem ist auch ein Zugriff auf das Einkommen von Angehörigen des Empfängers vorgesehen..$^{42}$ Es ist wohl kaum davon auszugehen, daß dies dem Pflegebedürftigen als "externer Effekt" seiner Entscheidung gleichgültig ist. Dann aber müßten Teile des Verwandteneinkommens dem jeweils eigenen hinzugerechnet werden, wodurch sich c.p. der Kreis derjenigen weiter verkleinert, die aufgrund zu geringen Einkommens rationalerweise vom Versicherungsabschluß absehen.

- Schließlich wird der Bezug von Sozialhilfe vielfach als "stigmatisierend" angesehen. Wenn dem so ist, wird der Empfang von Versicherungsleistungen dem von Sozialhilfemitteln selbst bei quantitativ gleicher Einkommenssituation vorgezogen. ${ }^{43}$

In Anbetracht dieser Diskrepanzen zwischen Modell und Wirklichkeit sowie der in den Abschnitten 2.2.1 und 2.2.2 genannten anderweitigen Hemmnisse am Markt für Pflegeversicherungen, die von BUCHOLZ und WIEGARD nur sehr eingeschränkt beachtet werden, ist deren These zu widersprechen, "die Ineffizienz der gegenwärtigen Regelungen" sei "ausschließlich (oder doch in erster Linie) auf einen Staatseingriff"44, nämlich die Sozialhilfe, zurückzuführen. Als ein Erklärungsstrang unter anderen ist der Sozialhilfe-Effekt gleichwohl von Belang.

Möglicherweise wirkt die Sozialhilfe noch in einem anderen Sinn hemmend auf die Bereitschaft zum Abschluß privater Pflegeversicherungen ein: Solange deren Träger nämlich - wie in der Bundesrepublik - in den meisten Fällen an der Finanzierung der Pflege beteiligt sind, bestimmen sie in Verhandlungen mit den Leistungserbringern die Pflegesätze und damit auch das Qualitätsniveau der Leistungen insgesamt. ${ }^{45}$ Es

\footnotetext{
${ }^{42}$ siehe dazu ausführlich A. Hänlein (1992)

${ }^{43}$ Vgl. dazu auch Rivlin/Wiener (1988), S. 65. Für die USA wird dort ein nennenswerter negativer Einfluß des in seiner Funktionsweise der Sozialhilfe analogen Medicaid-Programms auf die Versicherungsentscheidung verneint.

${ }^{44}$ W. Buchholz/W. Wiegard (1992), S. 453. Die Autoren folgern übrigens nicht, dieser Staatseingriff habe zu unterbleiben. Im Kern ist die Sozialhilfe als Notanker auch in ihrer Sicht nicht disponibel.

${ }^{45}$ vgl. DIW (1993), S. 421
} 
werden sich nämlich normalerweise auch die Leistungen einer privaten Pflegekostenversicherung nach den so bestimmten Pflegepreisen bemessen.

Damit aber wird eines der vorrangigen Versicherungsziele, die Gewährleistung eines den eigenen Präferenzen adäquaten Versorgungsniveaus, unerreichbar. Statt dessen schlagen sogar zukünftige Sparmaßnahmen, mit denen im Bereich der Sozialhilfe stets gerechnet werden muß, auf die Privatversicherungskunden durch. Ein Zwei-KlassenSystem auf dem Pflegemarkt, das diesen Zusammenhang - etwa in Form versicherungseigener Exklusivpflegeheime - aufbrechen könnte, erscheint wiederum erst auf Basis einer deutlichen Zunahme privater Verträge vorstellbar.

\subsection{Gründe für das Fehlen einer Sozialversicherung gegen das Pflegerisiko}

Es ist eine Reihe verschiedenartiger Phänomene genannt worden, die einer selbsttätigen Deckung des gegenüber dem Pflegefall anzunehmenden Sicherungsbedarfs am Markt entgegenstehen. Durch die Versicherung erzielbare Wohlfahrtsgewinne bleiben in dieser Lage unausgeschöpft, ein Eingreifen des Staates erscheint im Grundsatz angezeigt. Ergänzend sei nunmehr kurz diskutiert, warum eine solche Intervention in der herkömmlichen und beispielsweise für den Krankheitsfall gewählten Form der Errichtung einer Sozialversicherung in der Bundesrepublik Deutschland nicht längst erfolgt ist. ${ }^{46}$

Die Frage, warum die Pflegeproblematik nur in den Niederlanden zum Gegenstand einer Sozialversicherung geworden ist, kann hier und mit ökonomischen Mitteln nicht eingehend untersucht werden. Sie immerhin aufzuwerfen, erscheint im Lichte der oben vorgenommenen Charakterisierung des Pflegerisikos und der Analyse des Versicherungsmarktes dennoch geboten. Im Vordergrund steht bei den folgenden Überlegungen die Situation in der Bundesrepublik; wie weit die Übertragbarkeit auf andere Industriestaaten reicht, soll hier nicht erörtert werden. ${ }^{47}$

\footnotetext{
${ }^{46}$ Damit ist freilich in keiner Weise impliziert, daß dies die einzige oder die beste Form sei, die eine staatliche Intervention annehmen könnte.

${ }^{47}$ Auch ohne spezielle Sozialversicherung ist das Pflegerisiko in anderen westeuropäischen Staaten weniger ungedeckt als in der Bundesrepublik: Übernehmen mancherorts andere Sozialversicherungszweige immerhin partiell die Aufgaben einer Pflegeversicherung, so spielt in Ländern mit anderer sozialpolitischer Tradition die direkte staatliche Finanzierung von Pflegeleistungen mitunter eine große Rolle. Für Einzelheiten siehe M. Gabanyi u.a. (1992).
} 
In den vorangehenden Abschnitten der Untersuchung hat sich gezeigt,

- $\quad$ daß das Pflegerisiko (zumindest) ebenso "versicherungsbedürftig" ist wie das sonstige Krankheitsrisiko und

- $\quad$ daß einer Marktlösung ohne gezielten Staatseingriff erhebliche und vielfältige Hindernisse entgegenstehen.

Es ist daher auf den ersten Blick erstaunlich, daß der sehr umfassenden Abdeckung von Krankheitsrisiken im Wege der Sozialversicherung nicht eine entsprechende Regelung für den Pflegebereich gegenübersteht, zumal die dort offenkundig zweifelhafte Möglichkeit privatwirtschaftlicher Lösungen im Fall der Krankenversicherung zumindest unter den Bedingungen des deutschen Gesundheitssystems empirisch erwiesen ist. ${ }^{48}$

Gelegentlich wird im Vergleich zwischen Kranken- und Pflegeversicherung der letzteren die Eignung für eine Sozialversicherungslösung mit dem Argument abgesprochen, daß es sich aufgrund der Seltenheit des Pflegefalls nicht um ein "soziales Risiko" handele, sondern um ein "typisches Einzelfallrisiko". ${ }^{49}$ Abgesehen davon, daß die "Seltenheitsthese" mit Blick auf die am Anfang des Kapitels angeführten Zahlen kaum haltbar ist, erscheint ein solches Wahrscheinlichkeitskriterium insgesamt unsinnig. Niemand käme auf den Gedanken, allein angesichts sinkender Arbeitsunfallzahlen die Existenzberechtigung der Gesetzlichen Unfallversicherung infrage zu stellen. Als Merkmal von Sozialversicherungstatbeständen relevant ist die Allgemeinheit des Risikos selbst, nicht aber die seines Eintritts.

Ferner wird - insbesondere in der Diskussion um einen möglichen Prämienanteil der Arbeitgeber - ein direkter Bezug zum Arbeitsleben als Vorbedingung einer Sozialversicherungslösung eingefordert. Auch dieses Argument ist nicht stichhaltig. Ein kausaler Zusammenhang zwischen Berufstätigkeit und Risiko besteht allein in der Unfallversicherung, aber schon nur noch sehr bedingt in der Krankenversicherung. Könnte in bezug auf diese noch ins Feld geführt werden, daß sie (unter anderem) zur Wiederherstellung von Arbeitsfähigkeit dient, so läßt sich von der Gesetzlichen Rentenversicherung auch das nicht behaupten. Sie ist vielmehr Ausdruck des Anspruchs der Sozialversicherung, elementare Sicherungsbedürfnisse abhängig Beschäftigter gerade auch außerhalb ihrer Berufstätigkeit zu befriedigen. In diesem Sinn ließe sich eine

\footnotetext{
${ }^{48}$ Das oben beschriebene Problem der mangelnden längerfristigen Prognostizierbarkeit der Schadenskosten stellt sich freilich auch in der privaten Krankenversicherung und ist an der öffentlichen Debatte um die vielfach sehr starke Steigerung der Prämien im Alter deutlich geworden.
}

${ }^{49}$ T. Ruf (1985), zitiert nach R. Thiede (1990), S. 32 
soziale Pflegeversicherung nahtlos in den Begründungszusammenhang des bisherigen Systems einfügen.

Es lassen sich mithin - auf grundsätzlicher Ebene - keine sachlichen Gründe dafür finden, daß das Pflegerisiko vom System der Sozialversicherung ausgespart bleibt. Entscheidender sind dafür wohl erstens historische und zweitens politische Faktoren:

1. Das Pflegeproblem ist - bedingt durch medizinische Fortschritte und erhöhte Lebenserwartung - erst in jüngerer Vergangenheit in größerem Umfang aufgetreten und noch später - bedingt z.B. durch veränderte familiäre Lebensweisen - ins öffentliche Bewußtsein gerückt. ${ }^{50}$ Die Gründungsphase der Sozialversicherung, 1927 mit Einführung der Arbeitslosenversicherung abgeschlossen, lag zu diesem Zeitpunkt schon lange zurück.

2. Als das Problem in die öffentliche Wahrnehmung vorgedrungen war, bestanden bereits erhebliche Zweifel am Konzept der Sozialversicherung insgesamt und Widerstände gegen die mit ihr verbundenen Kostensteigerungen. Der Problem- und Meinungsdruck hat zwar nicht zur Abschaffung (oder auch nur zur gründlichen Reformierung) der bestehenden Systeme ausgereicht, wohl aber zur Verhinderung eines neuen. Noch in der Debatte zu Beginn der 90er Jahre war gelegentlich der Eindruck zu gewinnen, die diskutierte Pflegeversicherung fungiere geradezu als Stellvertreterin für das ebenso mißliebige wie reformresistente System der bestehenden Sozialversicherungszweige. ${ }^{51}$

\footnotetext{
so vgl. A. Prinz (1987), S. 40

${ }^{{ }_{1}}$ So spricht zum Beispiel der SVR (1991), Tz. 358 in bezug auf die Pflegefrage von einer "Entscheidung über den zukünftigen Kurs in der Ausgestaltung der sozialen Sicherung" insgesamt. Ähnlich auch der Wissenschaftliche Beirat beim BMF (1990), S. 17.
} 


\section{Das Ziel und die Maßstäbe einer gesetzlichen Regelung zur Absicherung des Pflegerisikos}

\subsection{Vorbemerkung zum generellen Ziel und zu seiner Begründung}

Die Erörterung der diversen Gründe dafür, daß der offenkundig vorhandene Sicherungsbedarf gegenüber dem Pflegerisiko am Markt nur sehr eingeschränkt zum Tragen kommt, führt zur Frage nach staatlichen Möglichkeiten zur Abhilfe. Bevor der in den Niederlanden beschrittene Weg untersucht und beurteilt werden kann, ist es zunächst erforderlich, das Ziel einer solchen Politik allgemein zu klären und zu operationalisieren. Ansätze dazu sind im folgenden zu entwickeln:

Generell darf unterstellt werden, daß Individuen aufgrund einer - wie stark auch immer ausgeprägten - Risikoaversion daran interessiert sind, für den Fall der Pflegebedürftigkeit vorzusorgen. Folglich besteht das generelle Ziel einer Pflegepolitikdarin, den Nachfragern in Überwindung der aufgezählten Hindernisse zur Erfüllung eines solchen Bedürfnisses zu verhelfen. In einem ersten Konkretisierungsschritt läßt sich der Vorsorgewunsch in eine finanzielle und eine materielle Komponente zerlegen: Einerseits gilt es Verlust von Einkommen und Vermögen aufgrund des Eintretens von Pflegebedürftigkeit auszuschließen, zum andern soll gewährleistet werden, daß das (eventuelle) Pflegebedürfnis selbst auf jeweils nach Form und Umfang adäquate Leistungsangebote trifft.

Für die Fundierung eines solchen Ziels kommen zwei verschiedene Motivationsmuster in Betracht: Zum einen könnte mit seiner Verfolgung eine allokative Verbesserung angestrebt werden, sofern die oben aufgeführten Hemmnisse den Tatbestand des Marktversagens erfüllen bzw. externe Effekte vorliegen. ${ }^{1}$ Zum anderen läßt sich die Versorgung im Pflegefall als Grundbedürfnis auffassen und deshalb fordern, daß deren Erlangung unabhängig von der Wohlstandsposition des Bedürftigen nur nach Bedarf zu erfolgen habe. Den Folgen dieses Unterschieds für die Bewertung von Lösungskonzepten braucht an dieser Stelle nicht nachgegangen zu werden; die im vorigen Absatz vorgenommene allgemeine Zielformulierung ist für objektiv-bedarfsbezogene und für

\footnotetext{
${ }^{1}$ W. Buchholz/W. Wiegard (1992), S. 449, betonen, daß es sich bei den von ihnen untersuchten Wirkungen der Sozialhilfe nicht um "irgendeine Form von Marktversagen" handelt, sondern um den Effekt eines staatlichen Eingriffs. Da dieser Eingriff aber unabdingbar ist, sei ein weitere staatliche Intervention zur Allokationsverbesserung erforderlich (ebenda, S. 453f.).
} 
eher individuell-präferenzmäßige Deutungen gleichermaßen offen. Auf diese Unterscheidung wird jedoch bald zurückzukommen sein; zunächst seien einige Merkmalsdimensionen erörtert, an denen entlang das Schutzziel spezifiziert werden kann.

\subsection{Zieldimensionen}

\subsubsection{Reichweite in bezug auf den versicherten Personenkreis}

Da dem Risiko, in einen Zustand der Pflegebedürftigkeit zu geraten, grundsätzlich alle Menschen ausgesetzt sind, sollte auch die Schutzwirkung eines Sicherungssystems niemanden aussparen, solange sich nicht Gruppen identifizieren lassen, die bereits in hinreichendem Umfang und verläßlicher Weise abgesichert sind. Eventuell könnte dies beispielsweise für solche Personen angenommen werden, die in großfamiliären Strukturen leben und mit ihren Angehörigen Vereinbarungen über häusliche Versorgung im Pflegefall getroffen haben. Auch in solchen seltenen bzw. - was das Vorliegen eines verbindlichen Vetrags betrifft - eher hypothetischen Fällen kann aber nicht ausgeschlossen werden, daß die Pflegebedürftigkeit in solcher Schwere eintritt, daß sie die Kapazität der Familie übersteigt. Ebenso ist es möglich, daß die zur Ausübung der Pflege vorgesehenen Personen zum Eintrittszeitpunkt nicht mehr am Leben oder einsatzfähig sind. Ein Restrisiko bleibt also unter allen Umständen offen.

Ferner ist an Personen zu denken, die kraft ihrer Berufstätigkeit (im Fall der Bundesrepublik etwa als Beamte) oder durch Abschluß einer privaten Versicherung bereits einen institutionalisierten Schutz genießen. In solchen Fällen wird jeweils zu entscheiden sein, ob die entsprechenden Arrangements in einem neu zu schaffenden Sicherungssystem aufgehen oder daneben fortbestehen sollen. Unter Gerechtigkeitsaspekten sowie zur Vermeidung von Koordinationsproblemen wird eine einheitliche Regelung zumindest als Zielvorstellung im allgemeinen attraktiver erscheinen; andererseits kann die korrekte Abwicklung bestehender Teilsicherungen mit einigem Aufwand sowie mit Nachteilen für die Angehörigen der betreffenden Gruppen verbunden sein. ${ }^{2}$ So erscheint etwa

\footnotetext{
${ }^{2}$ Vergleiche K. Dreher, Nur der Minister hüllt sich in Schweigen, Süddeutsche Zeitung vom 30.04.93. Dort ist von Befürchtungen die Rede, daß die Einführung der Pflegesozialversicherung in Deutschland letztlich daran scheitern könnte, daß sie im Zuge des Gesetzgebungsverfahrens von den damit befaßten Beamten sozusagen sabotiert würde. Mit der Einbeziehung in das neue System verlören die Beamten den recht weitgehenden Pflegeschutz, den ihr Dienstherr bislang beitragsfrei gewährt hat. Freilich ist dies eine Zeitungsanekdote, über deren Substanz hier nicht geurteilt werden kann.
} 
eine unkompensierte Entwertung privater Verträge durch die Einführung einer konkurrierenden Sozialversicherung nicht hinnehmbar. Diese Problematik wird hier nicht weiter verfolgt. Es gelte die Annahme, daß im Entscheidungszeitpunkt keinerlei spezifische Pflegesicherung existiert.

Schließlich könnten als bereits geschützt solche Personen gelten, deren Pflegekosten mangels Einkommen zur Gänze vom Mindestsicherungssystem im Sinne der Sozialhilfe getragen würden, das ihren Lebensunterhalt bereits vor Eintritt des Pflegefalls finanziert. Ein spezifisches System der Pflegesicherung würde grundsätzlich auch in solchen Fällen greifen und an die Stelle der allgemeinen Mindestsicherung treten. Letztere kommt ja ihrem Wesen nach erst dann in Betracht, wenn keinerlei anderweitige Ansprüche bestehen. Es ist allerdings festzuhalten, daß in derartigen Grenzfällen für die Betroffenen zumindest hinsichtlich ihrer finanziellen Lage die Einführung der Pflegesicherung keinen zusätzlichen Schutzeffekt entfaltet. Andererseits können den Betreffenden auch keine Finanzierungsbeiträge abgefordert werden, da sie definitionsgemäß am Existenzminimum leben. In anderem Zusammenhang wird auf diesen Aspekt noch einzugehen sein; für die hier behandelte Frage nach dem in ein Sicherungssystem einzubeziehenden Personenkreis ist er letztlich irrelevant.

Als Ergebnis kann unter der oben gemachten vereinfachenden Annahme festgehalten werden, daß ein Sicherungsmechanismus in bezug auf das Pflegerisiko möglichst die Gesamtbevölkerung umfassen sollte. Bei den dazu angestellten Überlegungen hat sich aber bereits abgezeichnet, daß der Schutzbedarf in Abhängigkeit von der familiären Situation oder dem Einkommen unterschiedliche Ausmaße annehmen kann. Dies leitet über zum zweiten Zielkriterium.

\subsubsection{Leistungsgrenzen: Leistungszugang und Selbstbeteiligung}

Hier geht es um die Frage, unter welchen Voraussetzungen und in welcher Höhe ein Sicherungssystem Leistungen zur Pflege gewähren soll. Möglichst ausgeblendet bleibt die damit eng verbundene Problematik der Leistungsformen ebenso wie die der Leistungsqualität; beide Aspekte werden in den anschließenden Abschnitten behandelt. In dieser Abgrenzung spitzt sich die Frage auf zwei Punkte zu: die Definition und Feststellung des Pflegefalls als Voraussetzung für den Leistungszugang einerseits, die Selbstbeteiligung der Pflegebedürftigen an den Kosten andererseits. 


\section{a) Leistungszugang}

Pflegebedürftig im physischen Sinn ist, wer für notwendige Alltagstätigkeiten, die gesunde Menschen allein ausüben können, fremder Hilfe bedarf. Pflegebedürftig-keit im sozialen Sinn liegt insoweit vor, wie bei Vorliegen physischer Pflegebedürftigkeit nicht Personen des nahen Umfeldes bereit und in der Lage sind, die erforderlichen Hilfsdienste zu verrichten. Ein System der Pflegesicherung hat dann für die Bereitstellung der entsprechenden Dienste bzw. dafür zu sorgen, daß der Betroffene selbst sie sich verschaffen kann.

Als allgemeine Bestimmung des Pflegefalls ist die obige Definition wohl hinreichend klar und unstreitig, auch wenn einige der verwandten Begriffe gewisse Interpretationsspielräume lassen. ${ }^{3}$ (Welche Verrichtungen können als "notwendig" gelten und von welcher Häufigkeit an handelt es sich um solche des "Alltags"?)

Problematischer ist die Frage des Verfahrens, nach dem der Bedarf festgestellt und sein Umfang ermittelt wird. Einerseits ist es ein Gebot der - später zu erörternden Effizienz, daß durch sorgfältige Prüfung von Hilfegesuchen ein Mißbrauch von Leistungen ausgeschlossen wird. Andererseits wird das Schutzziel nur dann vollständig erreicht, wenn es unter keinen Umständen zur Verweigerung benötigter Leistungen kommt. Es ist daher zu fordern, daß die mit der Indikationsstellung betrauten Instanzen neben (sozial-)medizinischer Kompetenz auch die nötige Unabhängigkeit sowohl von den Interessen des Versicherten als auch von denen der Versicherung aufweisen.

Ferner ist zu beachten, daß die altersbedingte Pflegebedürftigkeit auch die Fähigkeit oder die Bereitschaft einschränkt, die entsprechenden Leistungen zu beantragen. Es sollte daher die Möglichkeit bestehen, daß ein Prüfungsverfahren durch Dritte (Angehörige, Nachbarn, Hausarzt etc.) in Gang gesetzt wird.

\footnotetext{
${ }^{3}$ Von solchen Spielräumen wird mitunter so exzessiv Gebrauch gemacht, daß ihre Grenzen im Gegenzug eine rechtliche Klärung erfahren: Die Pflegeleistungen der deutschen Krankenkassen, wie sie seit 1989 existierten, waren an die Bedingung gekoppelt, daß der Bedürftige "in nahezu allen Bereichen des täglichen Lebens der Hilfe bedarf". Das Bundessozialgericht hat diese offenkundig lebensfremde Definition schwerer Pflegebedürftigkeit verworfen. Wenn der Hilfebedarf "für die gesamte Lebensführung des Versicherten prägend ist", sei es unerheblich, auf wieviele Lebensbereiche er sich bezieht. (Siehe Hamburger Abendblatt vom 31. Juli 1993: "Pflegegeld für mehr Behinderte" sowie die dort genannten Urteile.)
} 
Schließlich müssen die im Indikationsverfahren anerkannten Leistungsansprüche auch möglichst reibungslos einlösbar sein. Für ein Sachleistungssystem impliziert dies die Vorhaltung hinreichender Pflegekapazitäten. Ist diese Anforderung auch fast zu selbstverständlich, um einer Erwähnung zu bedürfen, so kann - wie sich zeigen wird ihre praktische Erfüllung durchaus zum Problem werden.

\section{b) Selbstbeteiligung}

Bevor dieser Punkt unter dem Blickwinkel der Effektivität beleuchtet werden kann, ist eine Begriffsabgrenzung vonnöten: Wenn das Schutzziel darin besteht, Betroffene von den aus ihrer Pflegebedürftigkeit resultierenden Lasten zu befreien, so ist es adäquat, eine Nettorechnung vorzunehmen. Es können also von den Gesamtkosten etwa eines Heimpflegeplatzes die Anteile in Abzug gebracht werden, die der Heimbewohner auch dann zu tragen hätte, wenn er nicht pflegebedürftig geworden wäre. Es sind dies die Kosten der normalen Lebensführung, sofern sie mit Eintritt der Pflegebedürftigkeit entfallen sind. Wenn derartige pflegebedingte Ersparnisse in korrekter Abgrenzung ermittelt ${ }^{4}$ und von der Erstattung ausgenommen werden, stellen sie keine echte Selbstbeteiligung dar und sind daher für die Realisation des Schutzziels von vornherein unschädlich.

Muß sich der Pflegebedürftige hingegen über eine solche Bereinigung hinaus an den Pflegekosten beteiligen, so schmälert dies den Versicherungsschutz. Abgesehen von den ohnehin nicht versicherbaren immateriellen Schäden erleidet er auch eine finanzielle Einbuße im Vergleich zum Gesunden, die durch das Sicherungssystem nurmehr gemildert wird. Sofern also davon ausgegangen werden kann, daß Versicherte Wert darauf legen, gerade im Pflegefall "wenigstens" in finanzieller Hinsicht schadlos

\footnotetext{
${ }^{4}$ Zur Klarstellung: "Korrekt" heißt hier strenggenommen individuell. Praktisch dürfte es unvertretbaren Aufwand erfordern und erhebliche Detailprobleme aufwerfen, die "Ersparnis" in jedem Einzelfall zu bestimmen. Das steht hier aber nicht zur Debatte. Die sog. "Hotelkosten" bei Heimpflege, die in den allermeisten in der deutschen Debatte kursierenden Vorschlägen zur Pflegesicherung als nicht erstattungsfähig vorgesehen sind, stellen im übrigen keineswegs eine pauschalierte Variante der Ersparnis im hier beschriebenen Sinn dar, sondern basieren (bestenfalls) auf der betriebswirtschaftlichen Kalkulation des Pflegeheims, deren Verhältnis zu den Kosten einer selbständigen Lebensführung außer Betracht bleibt (vgl. S. Winters (1991), S. 479). Insofern ist die Nichterstattung einer solchen Kostenkategorie eine irreführend etikettierte Form echter Selbstbeteiligung.
} 
gehalten zu werden, erscheint eine Vollabsicherung zunächst als Idealfall im Sinne des Schutzziels. ${ }^{5}$

Die Gültigkeit dieser Annahme setzt in technischer Formulierung allerdings voraus, daß das verfügbare Einkommen und der Gesundheitszustand als Variablen der Nutzenfunktion in einem Substitutionsverhältnis stehen. ${ }^{6}$ Verhalten sie sich hingegen komplementär zueinander, so wird eine Selbstbeteiligung mit Rücksicht auf die dadurch erzielbare Beitragsminderung gegenüber der Vollversicherung bevorzugt. ${ }^{7}$ Eine inhaltliche Erklärung dieser Variante könnte darin bestehen, daß ein Pflegebedürftiger in der Entfaltung seiner Bedürfnisse generell eingeschränkt sei, im Extremfall also mit seinem Einkommen "nichts mehr anzufangen weiß". Dies ist fall- bzw. teilweise sicher zutreffend ${ }^{8}$, aber sicher nicht unter allen Umständen. Immerhin kann dem durch die Gebrechlichkeit eingeschränkten Aktionsradius der Effekt gegenüberstehen, daß die noch vorhandenen Aktionsmöglichkeiten mit größerem finanziellen Aufwand verbunden sind (beispielsweise für Taxifahrten) oder sogar neue Bedürfnisse entstehen (Man denke etwa an eine behindertengerechte Wohnung bzw. Einrichtung).

Entscheidend ist nun, daß der Versicherungsnehmer im voraus nicht wissen kann, ob in seinem möglichen Pflegefall die kostentreibenden oder die kostensenkenden Veränderungen überwiegen werden. Diese Frage ist vielmehr selbst ein Teil des Risikos. Deswegen erscheint es letztlich doch plausibler, daß der typische Versicherungsnehmer bei idealtypischer Beitragsgestaltung ${ }^{9} \mathrm{im}$ allgemeinen die volle Kostendeckung wählen würde, um sich auf die "sichere Seite" zu begeben.

\footnotetext{
s vgl. etwa Buchholz/Wiegard (1992), S. 443. Dieser Befund setzt allerdings voraus, daß die Versicherung "faire" Prämien erhebt. Bei höheren Prämien kann eine nur teilweise Deckung vorgezogen werden (siehe H.-W. Sinn (1977), S. 515f. oder F. Breyer/P. Zweifel (1992), S. 178ff.). Ferner kommen auch moral-hazard-Effekte als Grund für den Wunsch nach Selbstbehalt in Betracht (siehe ebd., S. 191ff.). Deren Relevanz im vorliegenden Zusammenhang kommt im Verlauf der Effizienzanalyse ausführlich zur Sprache.

${ }^{6}$ In diesem Fall kann sogar eine "Überversicherung" vorteilhaft sein, die die Kosten zu mehr als $100 \%$ abdeckt. Diese wenig realistische Option wird hier nicht weiter beachtet.

${ }^{7}$ siehe ausführlich hierzu Breyer/Zweifel (1992), S. $187 \mathrm{ff}$.

${ }^{8}$ Dies übrigens sogar eher als im Fall der Krankenversicherung, auf den sich Breyer und Zweifel beziehen. Das dort angeführte Beispiel wirkt recht konstruiert (siehe ebd.).

${ }^{9}$ Liegen die Prämien oberhalb des "aktuarisch fairen" Niveaus, was in der Realität anzunehmen ist, so ist eine gewisse Selbstbeteiligung aus der Sicht des Versicherten erwünscht.
} 
In der Praxis wird ein obligatorischer Selbstbehalt aber vor allem zur Vermeidung von Fehlanreizen oder auch zur direkten Kostenbegrenzung zumindest für bestimmte Pflegeformen vielfach als notwendig angesehen. ${ }^{10} \mathrm{Im}$ hier vorliegenden Zusammenhang stellt sich dann die Frage, bis zu welcher Grenze die Erstattungspflicht der Versicherung reduziert werden kann, ohne deren Schutzwirkung substantiell auszuhöhlen.

Letztlich ist dies freilich eine Ermessensfrage; eine quantifizierte Antwort dürfte sich kaum begründen lassen. Es läßt sich nur vage postulieren, daß Sicherungsleistungen, die als solche bezeichnet zu werden verdienen, zum einen in einem angemessenen Verhältnis ${ }^{11}$ zur Höhe des (finanziellen) Gesamtschadens stehen und daß zum andern gewährleistet ist, daß der Restschaden im Verhältnis zum Einkommen seines Trägers keine existenzbedrohenden Ausmaße annimmt. Andernfalls könnten Leistungen, die durch Eintritt des Pflegefalls erforderlich werden, nicht mehr in vollem Umfang erworben werden; damit wären die Anforderungen des Schutzeffektes im Kern verletzt.

Es könnte gegen dieses Verdikt eingewandt werden, daß es praktisch zur Versagung benötigter Leistungen nicht kommen wird, da notfalls auf die Mindestsicherung zurückgegriffen werden könne. Aber selbst wenn davon ausgegangen wird, daß eine Zuzahlung, die die Möglichkeiten des Pflegebedürftigen übersteigt, in allen Fällen als Minimalbedarf im Sinne der jeweiligen Sozialhilfe-Bestimmungen Anerkennung fände, wäre eine solche Lösung äußerst unbefriedigend.

Wenn überhaupt ein Schutz vor den finanziellen Folgen eines spezifischen Schadensereignisses intendiert wird, erscheint es widersinnig, wenn Betroffene dennoch durch ebendieses Ereignis in eine Situation absoluter Armut gedrängt werden können. ${ }^{12}$

\footnotetext{
${ }^{10}$ Inwieweit diese Sicht berechtigt ist, wird im nächsten Kapitel kritisch zu prüfen sein.

${ }^{11}$ Wenn zum Beispiel weniger als die Hälfte der Kosten im Schadensfall gedeckt sind, wie dies vielfach bei der deutschen Pflegeversicherung vorkommen wird (siehe Kapitel 7), wäre eher von einer Art "Beihilfe" als von einer wirklichen und wirksamen Versicherung zu sprechen.

${ }^{12}$ Der Wissenschaftliche Beirat beim BMF (1990), S. 12, dessen Vorschläge zur Selbstbeteiligung derartige Fälle in großer Zahl programmieren, scheint diese Sicht zu teilen. Seine Auffassung, dieses Problem sei "im Rahmen der Rentenversicherung zu lösen", ist allerdings nicht nachvollziehbar, da die dort betrachteten Witwen nicht aufgrund einer für die normale Lebensführung unzureichenden Rente, sondern eben erst durch den Pflegefall in eine finanzielle Notlage geraten. Der Beirat fordert also, alle Rentner in der GRV prophylaktisch so zu stellen, daß sie die von ihm propagierte hohe Kostenbeteiligung tragen können. Erscheint dieses Vorgehen schon intuitiv wenig zielgenau und daher
} 
Damit verbunden sind aufgrund der bei Fürsorgeleistungen üblichen Bedürftigkeitsprüfung zunächst der Verlust evtl. vorhandenen Vermögens und danach auch die Heranziehung zahlungsfähiger Angehöriger. Es ist nicht anzunehmen, daß eine Versicherung, die solche Schadensfolgen nicht ausschließt, sich einer besonders hohen Akzeptanz und Beitragsmoral erfreuen wird.

Bedenken in Hinblick auf die Entwicklungsperspektiven der Versicherung kommen hinzu: Wird eine Kostenbeteiligung der Sozialhilfeträger nicht von vornherein ausgeschlossen, so kann es für einen Versicherungsträger reizvoll erscheinen, sich zu deren Lasten durch Manipulation des Selbstbehalts ${ }^{13}$ finanzielle Erleichterung zu verschaffen und die Sicherungslücken so noch weiter aufzureißen. Werden solche Tendenzen erkennbar und antizipiert, so sind es nicht mehr allein die in einem engen Sinn "Armen", sondern immer weitere Teile der Beitragzahlerschaft, die daran zweifeln müssen, daß die Versicherung sie im Bedarfsfall davor bewahrt, sozialhilfeabhängig zu werden.

Es erscheint sinnvoll, an der Forderung festzuhalten, daß ein taugliches Sicherungssystem sich zumindest dann bewähren muß, wenn die Folgen des Versicherungsfalles "katastrophale" Ausmaße in dem Sinn annehmen, daß sie die Leistungsfähigkeit des Betroffenen übersteigen.

\subsubsection{Leistungsinhalte und Leistungsform}

Vom oben diskutierten Aspekt des Deckungsgrades abzugrenzen ist die Frage, welche Leistungen überhaupt zum Katalog einer Pflegeversicherung gehören sollten. Dieses Problem überschneidet sich mit der Frage, ob die betreffenden Dienste selbst

\footnotetext{
unnötig kostspielig, so muß der Vorschlag erst recht deswegen verwundern, weil die Höhe der Selbstbeteiligung gerade als das bestimmt wird, was ein männlicher Arbeiter-Durchschnittsrentner gerade noch tragen kann. Somit impliziert die zitierte Anregung wenn schon keine völlige Nivellierung der (Arbeiter-)renten, so doch zumindest die Einebnung der Durchschnittsdiskrepanz zwischen Männern und Frauen, was in letzter Konsequenz einer Abschaffung des Beitragsbezugs in der Rentenversicherung gleichkäme.

${ }^{13}$ Dies ist besonders einfach, wenn nicht der Selbstbehalt, sondern die maximale Versicherungsleistung fixiert wird, so daß der jeweilige Rest als Eigenbeitrag der Versicherten anfällt. Die Verschiebung von Lasten auf die Sozialhilfeträger erfolgt dann bei nominalen Kostensteigerungen "automatisch", solange es nur unterlassen wird, die Leistungssätze anzupassen. Diese Gefahr birgt die künftige deutsche Pflegeversicherung.
} 
bereitgestellt oder zu deren Erwerb Geldleistungen gewährt werden. Beide Aspekte sind auch für die Effizienzdebatte von Bedeutung, sollen aber hier - der Struktur dieses Kapitels gemäß - zunächst unter dem Blickwinkel ihrer Zielwirkung betrachtet werden.

Die Überschneidung beider Fragestellungen kann wie folgt beschrieben werden: Wird grundsätzlich die Sachform gewählt, so ist direkt zu entscheiden, welche Leistungsarten in ein entsprechendes Sortiment für welche Fälle aufgenommen werden. Bei Pflegegeldern hingegen ist deren Aufteilung auf bestimmte Güter dem Empfänger anheimgestellt. Gleichwohl wird man auch in diesem Fall gewisse Normvorstellungen über das jeweils angemessene Leistungspaket entwickeln müssen, um die Transfersätze ggfs. unter Abzug einer Selbstbeteiligung- der Höhe nach korrekt bestimmen zu können.

Im folgenden soll daher zunächst erörtert werden, welche Leistungsinhalte als Bestandteile eines Schutzpakets in Betracht kommen. Von dort aus ist der sehr vielschichtigen und kontroversen Frage nachzugehen, ob monetäre Transfers ohne Zweckbindung oder Sachleistungen ${ }^{14}$ zur Realisation eines solchen Leistungskatalogs zweckmäßiger erscheinen.

\section{a) Das Spektrum der Pflegeleistungen}

Es leuchtet unmittelbar ein, daß Pflegebedarf kein homogenes Phänomen ist, sondern in einer großen Vielfalt von Abstufungen und Erscheinungsformen auftritt. ${ }^{15}$ Wie bereits im Kontext der Definition von Pflegebedürftigkeit insgesamt herausgestellt wurde, ist auch für den konkreten Bedarf nicht allein die physiologische Verfassung des Bedürftigen ausschlaggebend. Er ergibt sich vielmehr erst aus der Relation noch vorhandener Kompetenzen zu den Anforderungen der jeweiligen Lebenslage. Die für ein Leben ohne externe Hilfe erforderlichen Fähigkeiten sind aber in hohem Maß durch die familiale bzw. soziale Situation sowie durch materielle Umweltbedingungen be-

\footnotetext{
${ }^{14}$ Wesentlich für diese Unterscheidung ist nicht das formale Verfahren, sondern die Frage, ob der Leistungsanspruch in Geldeinheiten oder in Mengeneinheiten definiert ist. Eine Kostenerstattung nach Art der privaten Krankenversicherung fiele demnach ebenfalls in die Kategorie "Sachleistung". Nicht eigens betrachtet wird die Möglichkeit einer zweckgebundenen Geldleistung. Die meisten der auf die ungebundene Geldleistung bezogenen Argumente träfen auch diese Form.

${ }^{15}$ vgl. H. Braun (1987), S. 14f.
} 
stimmt. Zu den letzteren gehören die Lage und Beschaffenheit der Wohnung ebenso wie etwa die örtlichen Einkaufsmöglichkeiten und Verkehrsverhältnisse.

Für eine nähere Bestimmung des Pflegebedarfs ist also zu klären, in welchen Bereichen notwendiger Alltagsaktivität die betreffende Person unter den je spezifischen Bedingungen auf Hilfe angewiesen ist. ${ }^{16} \mathrm{Je}$ nach Art der dabei identifizierten Bedürfnisse läßt sich eine Einteilung vornehmen in die Gruppe der "körperbezogen Pflegebedürftigen" einerseits und in "Personen mit hauswirtschaftlichem oder sozialkommunikativen Hilfebedarf" andererseits. Eine solche Trennung erscheint sinnvoll in Hinblick auf die unterschiedliche Qualität der jeweils angezeigten Leistungen: Bei körperbezogenem Bedarf wird die Hilfsleistung im allgemeinen häufiger und mit größerem Aufwand, vielfach auch durch qualifiziertes Personal erbracht werden müssen.

Dennoch wird auch ein "nur" hauswirtschaftlicher Bedarf grundsätzlich als eine Variante des Versicherungsfalls anzusehen sein, da seine Deckung nicht minder unabdingbar ist und im Einzelfall mit kaum tragbaren finanziellen Lasten verbunden sein kann. Leben im Haushalt des Bedürftigen weitere Personen, die zu den entsprechenden Tätigkeiten in der Lage sind, liegt ein solcher Bedarf freilich nicht vor.

Am anderen Ende der Skala steht die fortgeschrittene und dauerhafte Schwerstpflegebedürftigkeit, die oftmals eine stationäre Unterbringung induziert. Diese gilt zwar aus guten Gründen als äußerste Notlösung; es liegt aber auf der Hand, daß sie sich nicht unter allen Umständen vermeiden läßt. Zu denken ist dabei z.B. an stark verwirrte Personen, die ständiger Aufsicht bedürfen. I.d.R. werden weder Angehörige noch professionelle ambulante Helfer dies zu vertretbaren Kosten auf Dauer leisten können. Daneben besteht in einer stationären Einrichtung prinzipiell eher die Chance, durch koordinierten Einsatz von Vertretern verschiedener Heil-und Pflegeberufe umfassende Betreuung zu gewähren und damit noch vorhandene Heilungs-, Aktivierungs- und Linderungsspielräume auszuschöpfen. ${ }^{17}$ Auch stationäre Pflege muß mithin im Leistungsspektrum einer Pflegesicherung vorgesehen werden, zumal sie regelmäßig mit einer extremen Kostenbelastung verbunden ist.

\footnotetext{
${ }^{16} \mathrm{vgl}$. Infratest (1992), S. 13

${ }^{17}$ Vgl. dazu R. Thiede (1990), S. 119. Thiede behandelt dort (S. 117ff.) auch sehr ausführlich und mit interdisziplinären Literaturhinweisen die Frage, ob auf stationäre Unterbringung gänzlich verzichtet werden sollte bzw. kann. Im Ergebnis wird die Frage auch dort verneint.
} 
Zwischen beiden Polen ist die ambulante Hilfe bei körperbezogenen Verrichtungen angesiedelt, die selbst wiederum eine Vielzahl unterschiedlicher Ausprägungen annehmen kann. Je nach Art der Behinderung und in Abhängigkeit vom Vorhandensein und der Einsatzbereitschaft weiterer Haushaltsmitglieder, wird insbesondere die Frequenz der regelmäßig erforderlichen Hausbesuche stark streuen. Unterschiedlich groß kann darüber hinaus der Bedarf an kurzfristigen Interventionen in Krisenfällen, an "Kontrollbesuchen" und Alarmbereitschaft sein. Ferner ist zu prüfen, ob über die bloße Versorgung hinaus auch therapeutische Elemente (z.B. Krankengymnastik oder Gehtraining) erwünscht und angezeigt sind.

Liegen also schon in der objektiven körperlichen und sonstigen Situation der Hilfebedürftigen große Unterschiede, denen ein effektives Sicherungssystem durch entsprechend differenzierte Leistungsangebote Rechnung tragen muß, so sind bei jeweils gegebener persönlicher Lage auch die Präferenzen des Hilfsempfängers zu berücksichtigen. So wird etwa die Bereitschaft, in ein Heim umzuziehen, auch davon abhängen, wie der Betroffene im einzelnen Grenzfall seine Bedürfnisse nach Autonomie und Sicherheit ${ }^{18}$, nach vertrauter Umgebung und nach Gesellschaft gegeneinander gewichtet. ${ }^{19}$ Aber auch innerhalb der oben unterschiedenen Betreuungsrubriken werden Individuen in vergleichbarer Lage unterschiedliche Wunschvorstellungen entwickeln. So wird es der eine vorziehen, sein "Essen auf Rädern" zu erhalten, während die andere sich - auch unter Mühen - lieber mit dem behilft, was sie selbst noch kochen kann, sofern ihr jemand den Einkauf abnimmt.

Ein Sicherungssystem ist offensichtlich umso effektiver, je flexibler es dieser Vielfalt unterschiedlicher Bedarfslagen, die zudem von individuellen Bedürfnissen überformt sind, gerecht wird. Es ist ja gerade die Selbstbestimmung, unter deren in gewissem Umfang unvermeidlicher Einschränkung Pflegebedürftige leiden. Mithin wird die Schutzwirkung einer Sicherung von den Betroffenen daran gemessen werden, inwieweit sie noch vorhandene Kompentenz respektiert und noch nutzbare Gestaltungsmöglich-

\footnotetext{
${ }^{18}$ vgl. H. Brandt (1987a), S. 33

${ }^{19}$ Kommt Pflege durch Angehörige in Betracht, konfligieren ferner der Wunsch nach Familienanschluß und das Bestreben, "der Familie nicht zur Last" zu fallen. In einer Repräsentativumfrage im Jahre 1989 schlossen sich gut $70 \%$ der Meinung an, als Pflegebdürftiger sei man am besten bei den Verwandten untergebracht. Immerhin gut ein Viertel der Befragten optierten hingegen unter Hinweis auf den oben genannten Wunsch für das Pflegeheim (siehe J. Alber (1990), S. 214).
} 
keiten offenhält. Dies impliziert, daß nicht nur unzulängliche, sondern auch übermäßige Pflege eine klare Zielverletzung darstellt.

Es sollten also die Breite des Spektrums im ganzen und die Wahlmöglichkeiten des Einzelnen im Rahmen des als notwendig Begründbaren maximiert werden. Bezieht man diese Feststellung auf die eingangs aufgeworfene Frage der Leistungsform, so erscheinen frei verfügbare Geldleistungen auf den ersten Blick überlegen, da sie dem Empfänger prinzipiell ein Maximum an Entscheidungsfreiheit gewähren. ${ }^{20} \mathrm{Im}$ folgenden Abschnitt gilt es diese These zu überprüfen.

b) Geldleistung vs. Sachleistung, Konsumentensouveränität vs. Konsumentenschutz

Ein erster Einwand gegen die soeben formulierte Plausibilitätsannahme beruht auf der Vermutung mangelnder Transparenz am Pflegemarkt. So argumentiert etwa THIE$\mathrm{DE}^{21}$, daß "kaum anders" als im Bereich medizinischer Leistungen "unzureichende fachliche Kompetenz" und "Informationsmängel" auf seiten der Nachfrager dazu führten, daß deren Marktverhalten nicht ihre Bedürfnisse widerspiegele. "Nur im Wege normativer Setzung durch entsprechend kompetente Instanzen" lasse sich daher das Pflegeziel bestimmen.

Eine solche, stark meritorische Sicht ist in derart pauschaler Form nicht gerechtfertigt. Haltbar erscheint sie allenfalls dort, wo die Pflegeleistungen stark medizinisch geprägt sind. Über Möglichkeiten und Qualität rehabilitativer Maßnahmen etwa mag sich der Pflegebedürftige oftmals im unklaren sein. Im Kernbereich der Pflege selbst hingegen ist eher SCANLON zuzustimmen, der - in ausdrücklicher Abgrenzung von "most other health care settings" - befindet: "Disabled persons can generally determine their own needs and the quality of services received. "22 Vermutlich, so läßt sich im Anschluß an die Überlegungen zum Zusammenspiel von Bedarf und Bedürfnis hinzufügen, können sie dies sogar weit besser als "kompetente Instanzen" aller Art.

\footnotetext{
${ }^{20}$ So z.B. W. Frank (1987), S. 235 oder auch P. Oberender (1986), S. 34. Die bei letzterem als Beispiel für Konsumentensouveränität vorgetragene Entscheidung, ob ein Pflegebedürftiger "zusätzlich zwei Stunden Pflege oder lieber eine Flasche Wein zur Linderung seiner Beschwerden nachfragen will", mutet allerdings - sicher ungewollt - zynisch an und erscheint eher geeignet, die Souveränität des Konsumenten im Pflegefall ad absurdum zu führen.
}

${ }^{21}$ R. Thiede (1990), S. 110

22 W.J. Scanlon (1992), S. 44. Ähnlich auch L. Paringer (1983), S. 122. 
Andererseits gilt auch diese These erklärtermaßen nur "generally", das heißt hier: mit Einschränkungen. In erster Linie ist die große Gruppe geistig verwirrter alter Menschen als Ausnahme zu nennen, die zwar in den meisten Fällen durchaus ein Empfinden für pflegerische "Qualitätsunterschiede" haben dürften, denen es aber an der Fähigkeit gebricht, dies und ihre Bedürfnisse mit Aussicht auf Gehör zu artikulieren. Erst recht sind sie außerstande, ihren Präferenzen durch entsprechendes Agieren am Markt Geltung zu verschaffen.

Darin, und weniger in einem grundsätzlichen Mangel an Urteilsvermögen in bezug auf Pflegeleistungen, liegt offenbar das zentrale Problem der gerade von Ökonomen ${ }^{23}$ häufig zugunsten einer Geldleistungslösung ins Feld geführten Konsumentensouveränität. Von solchen Einschränkungen mehr oder weniger stark betroffen sind nämlich offenbar nicht allein alte Menschen mit geistigen Handicaps. Schließlich setzt ein nutzenmaximierender Einsatz von Pflegegeldern am Markt die Beschaffung und kritische Auswertung von möglichst umfassenden Informationen über Preis- und Leistungsverhältnisse voraus. ${ }^{24}$ Aber schon etwa zur "Regelung finanzieller Angelegenheiten" sind laut INFRATEST nur gut ein Drittel aller dort als "Personen mit regelmäßigem Pflegebedarf" Definierten selbst imstande. ${ }^{25}$ Aber selbst Angehörigen einer solchen aktiven Minderheit wird es mitunter schwerfallen, aus inadäquater Pflege resultierendes Leid von solchem zu trennen, das aus der Pflegebedürftigkeit selbst erwächst und unvermeidbar ist.

Es kommt hinzu, daß ein Transparenzmangel in der Tat darin liegt, daß die Qualität einer Pflegeleistung ebenso wie die vieler anderer Dienste sich in vollem Umfang erst nach dem Kauf erweist. Der dann evtl. fällige Umstieg auf andere Anbieter kann mit erheblichen Transaktions- und Umstellungskosten verbunden sein und ist zudem mit erneuter Qualitätsunsicherheit verbunden. Gerade stationäre Pflegeleistungen sind insofern keine "Erfahrungsgüter". ${ }^{26}$

\footnotetext{
23 "At the heart of the economist's love affair with cash transfers is the doctrine of absolute consumer sovereignity." L.C. Thurow (1974), S. 193.

${ }^{24}$ U.E. Reinhardt (1990), S. 109, kritisiert an einer Äußerung BAUMOLS dessen "remarkable faith in the ability of frail, elderly persons to function as vigilant rational health care shoppers".

${ }^{25}$ Siehe Infratest (1992), Anhang, Tabelle 7. Von denjenigen mit "unregelmäßigem Pflegebedarf" unterhalb von "mehrfach wöchentlich" sind in diesem Punkt $20 \%$ auf Hilfe angewiesen.

${ }^{26} \mathrm{vgl}$. DIW (1993), S. $419 f$.
} 
Wenn solche Intransparenz besteht und nicht etwa über den Aufbau verläßlicher "Reputation" behoben werden kann, wird der Marktmechanismus tendenziell dafür sorgen, daß Leistungen höherer Qualität trotz entsprechender Bedürfnisse und - aus dem Pflegegeld gespeister - Zahlungsbereitschaften nicht mehr angeboten werden. Dies wiederum kann in einem zweiten Schritt zu einer zu knappen Bemessung der Pflegegelder führen, die sich ja nach beobachtbaren Marktpreisen richten werden. Damit würde ein unzureichendes Qualitätsniveau durch Restriktionen auf seiten der Nachfrage weiter verfestigt.

Die bisher genannten Argumente, das sei ausdrücklich betont, laufen noch nicht eigentlich auf eine meritorische Begründung von Sachleistungen hinaus. Die Betroffenen, so wurde unterstellt, kennen ihre ("wahren") Bedürfnisse durchaus; in Zweifel steht allein ihre Fähigkeit, deren Befriedigung den Spielregeln des Marktes gemäß auch durchzusetzen.

Es ist aber auch vorstellbar, daß die von Pflegebedürftigkeit Betroffenen ihren Bedarf vielfach systematisch unterschätzen. Eine solche Tendenz könnte etwa darin begründet sein, daß man den altersbedingten Verlust an Selbständigkeit und Leistungsvermögen nicht wahrhaben will. Auch können solche "Verfallserscheinungen" eine resignative Haltung fördern, die dann ebenfalls der aktiven Verfolgung im Grunde vorhandener Bedürfnisse im Wege steht. Schließlich ist zu berücksichtigen, daß das Alter als solches und damit auch die altersspezifischen Bedürfnisse in einer am Leitbild von Leistung und Dynamik orientierten Gesellschaft nach verbreiteter Einschätzung kein hohes Ansehen genießen. So herrscht zumindest unter jüngeren Menschen die Vorstellung vor, daß ein Leben im Alter nur insoweit wünschenswert sei, wie es von körperlichen und geistigen Einschränkungen frei bleibe. Andernfalls, so die gängige Meinung, wolle man lieber beizeiten sterben. Sofern alte Menschen solche Haltungen in Form eines "Minderwertigkeitsgefühls" verinnerlichen, ließe sich auch damit zugunsten einer Meritorisierung von Pflegeleistungen argumentieren.

Je größer das Ausmaß, indem die aufgeführten Momente eine "Zweckentfremdung" von Pflegegeldern bewirken, desto ausgeprägter ist auch die Gefahr, daß mangels wirksamer Nachfrage eine hinreichend dichte Angebotsstruktur im Bereich der ambulanten Pflegedienste nicht zustande kommt. Dies läßt sich etwa am Beispiel einer ständigen Rufbereitschaft illustrieren, die in einem eher dünn besiedelten Raum nur dann lohnend betrieben werden kann, wenn eine hinreichend große Anzahl Pflegebe- 
dürftiger daran partizipiert. Ist dies nicht der Fall, so betrifft die so entstehende Lücke im Betreuungssystem auch diejenigen, die für solche Dienste zu zahlen bereit wären.

Eine ambivalente Rolle spielen in bezug auf die Frage der Leistungsform die Angehörigen des Pflegebedürftigen bzw. auch sonstige Personen, die ihm nahestehen. Wenn sie als ideale Treuhänder des bedürftigen Familienmitglieds agieren, lassen sich beinahe $^{27}$ alle oben genannten Einwände gegen die Gewährung von Leistungen in Geldform zerstreuen. Grundsätzlich können Angehörige (in bezug auf die erste Gruppe von Argumenten) den Pflegebedürftigen im Verhältnis zu den Leistungsanbietern so vertreten, daß dessen eingeschränkte "Marktfähigkeit" nicht mehr ins Gewicht fällt und die Meritorisierung quasi selbst insoweit vornehmen, wie es ihnen gelingt, dem Pflegebedürftigen seine verschütteten Ansprüche bewußt zu machen.

Ebenso können Angehörige - neben den Leistungsanbietern - aber auch zu denen gehören, die sich die eingeschränkte Souveränität des Pflegebedürftigen in Verfolgung eigener Interessen zunutze machen. Schließlich ist davon auszugehen, daß ihnen die nicht verbrauchten Teile des Pflegegeldes vielfach entweder direkt oder als späteres Erbe zufließen werden. Zugespitzt tritt diese Fallunterscheidung in der Frage auf, ob es ratsam ist, Angehörige für selbstgeleistete Pflege zu entlohnen. Eine Entscheidung für die Geldform impliziert diese Möglichkeit als Resultat einer entsprechenden Übereinkunft zwischen dem Pflegegeldempfänger und seinen privaten Betreuern. Im Falle intakter familiärer Beziehungen und auf Basis einer beiderseits freien Entscheidung wird ein solches Arrangement vielfach eine gute Lösung sein.

Andererseits sind Mißbräuche infolge einer überwiegend egoistischen Motivation der Angehörigen keineswegs auszuschließen. Realistischerweise ist nicht davon auszugehen, daß der Bedürftige seinen Verwandten das Pflegegeld zugunsten des Ankaufs professioneller Dienste entzieht, wenn ihm die dafür gebotene Gegenleistung unzureichend erscheint. Zu groß und vielschichtig sind insbesondere in einem gemeinsamen Haushalt, aber nicht nur dort, die Drohpotentiale der Pflegepersonen.

Aber auch außerhalb von worst-case-Szenarien lassen sich Bedenken gegen die Bezahlung von Betreuungsdiensten innerhalb der Familie formulieren. Da sie nämlich

\footnotetext{
${ }^{27}$ Das Problem fehlender Produkterfahrung ist auch durch die Angehörigen nicht ohne weiteres lösbar. In gewissem Maß gegensteuern können sie indes auch hier, indem sie die Kosten des Anbieterwechsels, etwa beim Umzug in ein anderes Pflegeheim, tragen helfen.
} 
kaum "marktgerecht" erfolgen kann, stellt sie eine Verwischung der Grenze zwischen freiwilliger und erwerbsmäßiger Tätigkeit dar und kann dazu führen, daß Pflegepersonen ihre eigene Motivationslage unklar wird. ${ }^{28}$

Aus der Sicht des Effektivitätskriteriums wird sich eine klare und restlos befriedigende Entscheidung zugunsten der einen oder anderen Leistungsform nicht fällen lassen. Auch eine Mischlösung beider Formen je nach Leistungsart vermag im Lichte der aufgeführten Argumente nicht recht zu überzeugen. ${ }^{29}$ Eher schon die Einräumung der Möglichkeit für den einzelnen, für eine der beiden Formen zu optieren. Ein Teil der gegen die Gewährung von Geldleistungen aufgeführten Bedenken gilt dann allerdings immer noch - nunmehr statt auf die Marktentscheidungen auf die Ausübung des Optionsrechtes selbst bezogen.

Einerseits erscheint die Geldlösung nicht nur aus allgemeinen wirtschaftstheoretischen Erwägungen, sondern auch angesichts der spezifischen, vor allem subjektiven Vielfalt der Bedürfnisse nach Pflegeleistungen zunächst als adäquat. Andererseits sind gegen diese Gestaltungsvariante gewichtige Einwände vorgetragen worden. Ein Sachleistungssystem $^{30}$ könnte daher als überlegen eingestuft werden, wenn es gelingt, die mit ihm verbundenen Nachteile in engen Grenzen zu halten. Anzustreben wäre die Erfüllung der folgenden Bedingungen:

- $\quad$ Die überhaupt angebotenen bzw. erstattungsfähigen Leistungen müßten ein breites Spektrum umfassen.

- Diese Palette sollte unkonventionelle Lösungen im Einzelfall ebenso ermöglichen oder ermutigen wie generelle Innovationen. ${ }^{31}$

\footnotetext{
${ }^{28}$ In Anwendung auf die modellhafte Beschreibung der familiären Entscheidungslage durch P. Zweifel et al. (1994), Kap. 4, würde diese Überlegung besagen, daß der "Nutzen aus altruistischer Handlung" nicht - wie dort impliziert - unabhängig von den finanziellen Anreizen ist, so daß beide Faktoren quasi addiert werden könnten.

${ }^{29}$ Siehe etwa R. Thiede (1990), S. 210, wo Sachleistungen im stationären, Geldzahlungen für den ambulanten Bereich vorgeschlagen werden.

${ }^{30}$ Zur Klarstellung: Die Gewährung der Leistungen in Sachform impliziert nicht, daß der Versicherungsträger oder gar "der Staat" deren Erstellung selbst vornimmt. Es wird hier vielmehr unterstellt, daß die Versicherung die Leistungen bei beliebig verfaßten Trägern "einkauft" und an die Bedürftigen "weiterreicht".

${ }^{31}$ G. Igl (1986), S. 199, traut solche Flexibilität einem Sachleistungssystem nicht zu und lehnt es deswegen $a b$.
} 
- $\quad$ Das System müßte rasch und unbürokratisch reagieren können, wenn sich die Verfassung oder die Wünsche des Pflegebedürftigen im Zeitverlauf ändern. ${ }^{32}$

- Auch innerhalb einer Pflegeart sollte für Anbietervielfalt gesorgt und im Rahmen des Möglichen ${ }^{33}$ sichergestellt werden, daß dem Pflegebedürftigen nicht nur einmalig, sondern fortgesetzt die Möglichkeit verbleibt, zwischen konkurrierenden Anbietern zu wählen. Dies schüfe für die Leistungserbringer zugleich einen Qualitätsanreiz (im Unterschied zur bei Geldleistungen drohenden reinen Preiskonkurrenz).

- Da auch in diesem Fall auf die Wirksamkeit der Sanktionsfunktion des Nachfragerverhaltens allein nicht gebaut werden kann, wären zusätzlich Qualitätsstandards zu setzen und zu überwachen. ${ }^{34}$

\subsubsection{Leistungsqualität}

Der Aspekt der Qualität von Pflegeleistungen ist in den bisherigen Erörterungen bereits mehrfach angeklungen. Wenn er hier dennoch eigens aufgeführt wird, so geschieht das nicht in der Absicht, detaillierte Kriterien dafür zu entwickeln, wann Pflege als "ausreichend", "angemessen" oder "hochwertig" anzusehen ist. Diese Aufgabe muß den Fachleuten für Gerontologie, Sozialmedizin und Pflegekunde vorbehalten bleiben. Vielmehr geht es zunächst allein darum, die Bedeutung der Leistungsqualität als wichtiger Zielkomponente hervorzuheben, zumal dies in der ökonomisch geprägten Pflegedebatte vielfach unterbleibt. Zudem soll versucht werden, einige grobe Maßstäbe aufzufinden, mittels derer Qualitätsniveaus auch für sozialmedizinische Laien faßbar werden.

Ein Qualitätsanspruch ist direkt ableitbar aus dem eingangs formulierten generellen Ziel der Versicherung, für den Pflegefall finanziell und versorgungsmäßig vorzusorgen. Maßgeblich für die erwünschte Schutzwirkung ist damit neben dem Ausmaß der finanziellen Absicherung und dem Katalog der für den Versicherungsfall versprochenen

\footnotetext{
32 vgl. R. Thiede (1990), S. 210

${ }^{33}$ Bei der Heimpflege ist dies - wie gesagt - nur eingeschränkt praktikabel.

${ }^{34}$ Dies ist stets schwierig und kostspielig und wäre andererseits im Grundsatz auch im Rahmen der Geldlösung vorstellbar. Dort allerdings würde die Durchsetzung von Qualitätsnormen als Eingriff in die Vertragsfreiheit zwischen Anbietern und Bedürftigen wohl auf größere Widerstände stoßen. Werden hingegen Sachleistungen gewährt, so fungiert der Versicherungsträger selbst als Vertragspartner und kann gegen stärker standardisierte Tarife bestimmte Leistungsniveaus direkt einfordern.
} 
Leistungsarten und -formen ${ }^{35}$ auch die Beschaffenheit der Leistungsinhalte im einzelnen. Es läßt sich sogar die These vertreten, daß letzterer Aspekt von den Betroffenen bzw. von den Gesicherten als potentiell Betroffenen als der wesentlichste angesehen wird. Dies soll zunächst für den Sektor der stationären Versorgung genauer ausgeführt werden.

Von der Option, nötigenfalls einen Heimpflegeplatz in Anspruch nehmen zu können, wird nur dann ein Optionsnutzen ausgehen, wenn die stationäre Unterbringung nicht unter allen Umständen als ein großes Übel angesehen wird. Denn während der ebenfalls unerwünschte - Gang in ein Krankenhaus nur als Mittel zum Zweck einer möglichst baldigen Heilung dient, hat das Pflegeheim den Charakter einer Lebensumwelt, die der Bewohner nur noch selten und in vielen Fällen nur noch vorübergehend verlassen wird. Als ein Übel aber, das der Realisierung attraktiver Nutzenniveaus kaum Raum bietet, werden Pflegeheime vielfach empfunden. ${ }^{36}$ Nun ist diese Abneigung sicherlich teilweise unaufhebbar, sofern sie sich z.B. auf den Verlust einer vertrauten vorherigen Umgebung bezieht.

Andererseits ist mit gleicher Gewißheit davon auszugehen, daß die Qualität der Heime, wie sie von den Versicherten wahrgenommen oder eingeschätzt wird, von erheblichem Einfluß ist. Dafür spricht auch das Ergebnis einer Umfrage unter älteren Menschen aus dem Jahre 1975, bei der die Forderung nach mehr und besseren Alten- und Pflegeheimen zu den am häufigsten genannten Wünschen an die Altenpolitik gehörte. ${ }^{37}$ Würde die Institutionalisierung als solche rundweg abgelehnt, hätte eine solche Forderung keinen Sinn.

Was aber ist ein "gutes" Pflegeheim, woran bemißt sich seine Qualität? Abstrakt formuliert läßt sich sagen, daß die Pflege umso zielgerechter ist, je wirksamer sie die unerwünschten Folgen zum einen der Pflegebedürftigkeit selbst, zum andern der institutionalisierten Unterbringung in Grenzen hält. Diese zu minimierenden Folgen sind neben dem eher medizinischen Aspekt einer Beeinträchtigung des körperlichen Wohlbefindens insbesondere Einbußen an der Fähigkeit, ein selbstbestimmtes Leben

\footnotetext{
${ }^{35}$ Die im vorigen Abschnitterörterte Differenziertheit und flexible Verfügbarkeit eines solchen Angebots kann selbst als übergeordnetes Qualitätsmerkmal angesprochen werden.

${ }^{36}$ siehe etwa E.-M. Dennebaum/W. Rückert (1987), S. 163f.

${ }^{37}$ K.G. Tismer u.a. (1975), S. 76
} 
zu führen. Solche Restriktionen ergeben sich sowohl aus der psycho-physischen Lage der Heimbewohner als auch aus der Wohnform. Die Lockerung oder Aufhebung der Beschränkungen kann auf drei Ebenen ansetzen: ${ }^{38}$

- Das anspruchsvollste Ziel bestünde darin, die Pflegebedürftigkeit durch rehabilitative Programme so weit herabzusetzen, daß der Verbleib im Heim nicht länger erforderlich ist. ${ }^{39}$

- Unterhalb dieser Ebene zielt eine "aktivierende Pflege" ${ }^{40}$ darauf ab, in bezug auf einzelne Fähigkeiten schon eingetretene Kompetenzverluste rückgängig zu machen.

- Wenn auch solche Fortschritte nicht erzielt werden können, geht es darum, die noch vorhandenen Handlungsmöglichkeiten des Bedürftigen zu identifizieren und ihm die zu deren selbstbestimmter Entfaltung nötige Hilfestellung in richtiger Dosierung und flexibler Weise zu gewähren. ${ }^{41}$ Durch Pflege dieser Art können weitere Kompetenzverluste, etwa eine drohende Bettlägerigkeit, vermieden werden.

Wenn nicht einmal der zuletzt genannte Anspruch erfüllt wird, ist bereits die Grenze zur "gefährlichen Pflege" überschritten, zu deren Erscheinungsformen RÜCKERT neben der "vermeidbare(n) Bettlägerigkeit" unter anderem auch "unangemessene Essenszeiten, zu lange Nächte (und) Beruhigungsmittelmißbrauch" zählt. ${ }^{42} \mathrm{Die}$ Vermeidung solcher Gepflogenheiten stellt hinsichtlich der Qualität stationärer Pflege ein Minimum, nicht aber eine Selbstverständlichkeit ${ }^{43}$ dar.

\footnotetext{
${ }^{38}$ S. Gößling (1987), S. 187, bestimmt in ähnlicher Weise das Ziel "gerontologisch fundierte(r) Rehabilitation" die "Störungen, Schäden, Abbauerscheinungen soweit wie möglich wieder rückgängig machen, sonst aber eine Verschlimmenung verhindern und immer das Zurechtkommen mit der gegebenen Situation herbeiführen" wolle.

${ }^{39}$ Die Notwendigkeitvon Rehabilitationsmaßnahmen betont auch die Bundesregierung (vgl. BundestagsDrucksache 10/2784, S. 18).

${ }^{40}$ siehe H. Brandt (1987), S. 123

${ }^{41}$ vgl. etwa bei T. Klie (1987), S. 54, die Kritik an üblichen Zwangsmaßnahmen, die dort zwar nicht ausschließlich, aber auch auf mangelnde finanzielle Ausstattung der Heime zurückgeführt werden.

${ }^{42}$ W. Rückert (1987a), S. 113

${ }^{43}$ Für die Bundesrepublik siehe etwa H. Brandt (1987), S. 124f. bzw. die dort zitierte Literatur.
} 
Es liegt auf der Hand, daß die Erfüllung der genannten Kriterien in erster Linie eine Frage der Personalausstattung von Pflegeheimen ist. Nicht allein erfordern die aufgeführten rehabilitativen, aktivierenden und Beistandsleistungen selbst erheblichen Zeitaufwand; vielmehr setzen sie die aufmerksame und reflektierte Beobachtung der Patienten voraus, für die ebenfalls zeitlicher Spielraum vorhanden sein muß. Schließlich ist zu beachten, daß der Wunsch, die Bedürfnisse der Einzelnen möglichst weitgehend zu respektieren, der Rationalisierung von "Betriebsabläufen" in der Pflege enge Grenzen setzt. $^{44}$

Entscheidende Voraussetzung für hochwertige und bedarfsgerechte Heimpflege ist somit zunächst die Zahl der Arbeitsminuten, die im statistischen Durchschnitt pro Tag und Patient für die personenbezogene Pflege und Betreuung eingesetzt werden können. Diese Größe ist bei gegebener tariflicher Arbeitszeit und einem gegebenen Quantum "bewohnerferner Aufgaben" eine Funktion der Pflegepersonalstärke pro Bewohner. Auch wenn für eine präzise Qualitätsmessung im Einzelfall zu prüfen wäre, ob "die verfügbare Zeit tatsächlich den Bewohnerinnen und Bewohnern zugutekommt"45, erscheint es für die hier verfolgten Zwecke angebracht, die Ausstattung mit Pflegepersonal als zentralen Gütemaßstab festzuhalten.

Bei der Festlegung entsprechender numerischer Richtwerte, die hier nicht unternommen wird, wäre allerdings auch die durchschnittliche "Schwere" bzw. Betreuungsintensität der Pflegefälle in Rechnung zu stellen. Die Leistungsfähigkeit eines Sicherungssystems unter dem Aspekt der Heimqualiät bemißt sich somit auch danach, ob steigenden Anforderungen pro Fall, wie sie etwa aus bestimmten Formen medizinischen

\footnotetext{
${ }^{4}$ Zur Illustration kann dabei etwa die in Pflegeheimen vielfach beobachtbare Tendenz dienen, mit einer relativ starken Frühschicht die meisten Aufgaben in den Morgenstunden geballt zu erledigen, um für den Rest des Tages sowie insbesondere am Wochenende die Schichtbesetzung und damit die Betreuungsangebote auf ein Minimum zu reduzieren. Dies mag betriebswirtschaftlich sinnvoll erscheinen, schon weil das Personal in der Regel den werktäglichen Frühdienst vorzieht und für andere Dienstpläne höher entlohnt werden müßte. Die Entfaltungsspielräume für die Hilfsbedürftigen werden durch ein solches Verfahren aber in bedenklicher Weise eingeschränkt (siehe zu letzterem W. Rückert (1987a), S. 114).
}

${ }^{45}$ ebd. S. 117 
Fortschritts oder auch aus einer Verbesserung ambulanter Dienste ${ }^{46}$ erwachsen können, in geeigneter Weise durch verbesserte Personalschlüssel begegnet wird.

Von augenfälliger Bedeutung ist ferner der Anteil des pflegerisch oder funktionsspezifisch qualifizierten Personals. ${ }^{47}$ Dabei steht zunächst außer Frage, daß die Behandlungspflege in bezug auf Krankheiten ebenso wie weite Teilbereiche der rehabilitativen und der aktivierenden Pflege überhaupt nur von geschulten Pflegekräften vorgenommen bzw. angeleitet und überwacht werden können. Darüber hinaus ist aber wichtig zu betonen, daß das Personal im Pflegesektor auch bei technisch anspruchslosen Verrichtungen vielfach mit normabweichendem psychisch-geistigem Verhalten der Bewohner konfrontiert wird. Geriatrisches Wissen um die Hintergründe solcher Phänomene kann entscheidend zu der Fähigkeit beitragen, adäquat mit ihnen umzugehen. ${ }^{48}$

In Hinblick auf den baulichen Standard stationärer Einrichtungen ist es insbesondere wichtig, in welchem Umfang Einzel- oder Zweibettzimmer vorhanden sind. Die Unterbringung in größeren Einheiten läuft dem Wunsch der Pflegebedürftigen nach Wahrung einer privaten Sphäre zuwider. ${ }^{49}$

46 Ein solcher Zusammenhang besteht dann, wenn in zunehmendem Umfang auch schwerer Pflegebedürftige daheim betreut werden können und infolgedessen nur noch in besonders schwierigen Fällen eine Heimeinweisung erfolgt (vgl. etwa Arbeitsgemeinschaft der Spitzenverbände der freien Wohlfahrtspflege des Landes Nordrhein-Westfalen (1986), S. 1).

${ }^{47}$ Ein besonders krasses Beispiel für die Mißachtung dieses Kriteriums lieferte der Präsident des Bundesverbandes des Deutschen Groß- und Einzelhandels mit seinem Vorschlag, es sollten "statt einer umlagefinanzierten Pflegeversicherung (..) Sozialhilfeempfänger im Pflegedienst eingesetzt werden" (siehe Handelsblatt vom 19. April 1993: "Gesetz zur Bekämpfung der Sozialkriminalität verlangt"). Mag man diese Äußerung eines sachfremden Interessenvertreters auch als Kuriosität einstufen, so ist die darin auf die Spitze getriebene Tendenz in der Bundesrepublik durchaus von Relevanz: Ebenfalls im Frühjahr 1993 wurde das Vorhaben der Bundesregierung bekannt, im Heimbereich eine verstärkte Delegation betreuender Tätigkeit an Hilfskräfte zu ermöglichen und die Vorschrift aufzuheben, wonach auch nachts auf Pflegestationen eine Fachkraft anwesend sein muß (siehe SZ vom 8. Mai 1993: "Heime sollen Fachpflege nicht Hilfskräften übertragen").

${ }^{48}$ Diese Feststellung beruht auf eigenen Erfahrungen des Verfassers aus seiner Tätigkeit im Pflegebereich. E.-M. Dennebaum und W. Rückert (1987), S. 173, treffen sie analog in bezug auf pflegende Angehörige.

${ }^{49}$ Eine entsprechende Forderung ist auch in der Debatte des Bundestags um die Pflegeversicherung erhoben worden (siehe Das Parlament (1993), S. 7). 
Die für den stationären ${ }^{50}$ Bereich entwickelten Anhaltspunkte zur Erfassung von Pflegequalität als wesentlicher Zielgröße lassen sich auf die ambulante Pflege - mutatis mutandis - übertragen. Die Wirkung des Personalbestandes auf die Leistungsgüte ist hier noch direkter, da die ambulante Pflegekraft ihre Nettoarbeitszeit unter den ihr zugewiesenen Klienten überschneidungsfrei aufteilt und die Gesamtleistung sich innerhalb des so bestimmten Zeitrahmens abspielt. Es werden mithin in aller Regel verbindliche Normen für die maximale Besuchsfrequenz und -dauer pro "Fall" bestehen. Ersterer Grenzwert ist mitentscheidend dafür, in welchen Fällen der meist unerwünschte Übergang in die stationäre Pflege vermieden werden kann. Von der vorgesehenen Besuchsdauer hängt ab, inwieweit rehabilitativ und aktivierend gearbeitet werden und auch ein eventueller Kontaktbedarf befriedigt werden kann. ${ }^{51}$ Neben dem Niveau dieser Grenzwerte wird die verfügbare Reservekapazität von vorrangiger Bedeutung sein. Sie entscheidet darüber, in welchem Umfang auf Schwankungen im individuellen Bedarf Rücksicht genommen werden kann. ${ }^{52}$

\subsection{Spezifikationen und Änderungen am Zielkatalog bei Einstufung der Pflege- leistungen als Grundbedarfsgüter.}

Im Zuge der Vorbemerkungen ist unter Berufung insbesondere auf THUROW die Möglichkeit erwähnt worden, der Befriedigung eines Pflegebedarfs als Komponente der Gesundheitsversorgung den Status eines menschlichen Grundrechts zu verleihen. Ähnlich argumentiert auch DANIELS, wenn er die RAWLSsche Theorie der Gerechtigkeit auf den Bereich der Gesundheitsfürsorge auszudehnen und anzuwenden sucht: Er definiert Krankheiten als objektiv faßbare "deviations from the natural functional organization of a typical member of a species" ${ }^{53}$ und erblickt darin eine zufällig, also ungerecht verteilte Einschränkung von Wahlmöglichkeiten. Die Forderung nach einer Korrektur dieser Ungleichheit bzw. ihrer finanziellen Folgen ist demnach ein Gerechtigkeitsgebot im Sinne RAWLS': Unter dem "Schleier der Unwissenheit" über ihre eigene gesundheitliche Konstitution würden sich die Menschen für einen

\footnotetext{
${ }^{s 0}$ Siehe ergänzend zur (möglichen) Bedeutung teilstationärer Dienste S. Gößling (1987).

s1 vgl. Dennebaum/Rückert (1987), S. 173

\$2 vgl. Bundestags-Drucksache 10/2784, S. 18: "Ambulante Dienste müssen im Notfall absolut zuverlässig und ausreichend zur Verfügung stehen."

${ }^{53}$ N. Daniels (1981), S. 155
} 
Ausgleich gesundheitlicher Nachteile aussprechen. ${ }^{54}$ Erst nach einer solchen Herstellung gleicher Startchancen kommt die leistungsgerechte Verteilung von Einkommen und Wahlmöglichkeiten über den Marktmechanismus zum Zuge.

Laut DANIELS ist die außermarktliche Korrektur der Verteilung von Gesundheit auch dann angezeigt, wenn eine vollständige Wiedererlangung der körperlichen Funktionsfähigkeit unmöglich ist; demgemäß bezieht er die Altenpflege ausdrücklich und als gleichwertig in sein Konzept ein. ${ }^{55}$ Ein solcher Ansatz impliziert die Forderung, daß die Verteilung benötigter Pflegegüter gänzlich außerhalb des Marktgeschehens allein nach Bedarfsgesichtspunkten zu erfolgen hätte. Das Sicherungssystem wäre dann so zu gestalten, daß die Versorgung mit solchen Gütern in jeweils hinreichendem Umfang unabhängig von der individuellen Einkommens- und Vermögensposition gewährleistet wäre. ${ }^{56}$ Bei Gültigkeit einer solchen Zielvorstellung ${ }^{57}$ lassen sich die oben unter 3.2 erörterten Effektivitätskriterien in einigen Punkten konkreter fassen:

ad 1. (Reichweite): Die in der allgemeinen Betrachtung nur schwach begründete Forderung nach Einbeziehung der Gesamtbevölkerung in das Schutzsystem gilt nunmehr in dezidierter Form. Bei Existenz unterschiedlicher Sicherungsformen wäre zu erwarten, $\mathrm{da} ß$ sich Zugangsprivilegien für bestimmte Gruppen herausbilden.

ad 2. (Leistungsgrenzen): Für die unter 2a) abgehandelte Frage der Abgrenzung leistungsauslösender Tatbestände ergeben sich im Kern keine Veränderungen. Der Leistungskatalog des Sicherungssystems hätte alles zu umfassen, woran ein nach gesellschaftlicher Übereinkunft "zwingender" und in der Hilflosigkeit begründeter Bedarf besteht. Eine Selbstbeteiligung, wie im Punkt 2b) diskutiert ist auf Basis der hier verfolgten Zielsetzung im Grundsatz abzulehnen, da es begüterten Personen leichter fällt, eine solche zu tragen. Eine einkommensunabhängige Versorgungssicherheit ist nicht mehr uneingeschränkt gegeben. Ein solcher, im hier angesprochenen Sinn

\footnotetext{
${ }^{54}$ Rawls selbst hat diesen Aspekt damit ausgeblendet, daß er für seine Betrachtung "normal active and fully cooperating members of society" unterstellt (zitiert nach N. Daniels (1981), S. 164).

ss siehe ebd., Seiten 168 und 178

${ }^{56}$ Schwierig ist es dabei, die Grenzen des Grundbedürfnisses abzustecken. Selbstverständlich verlangt die hier referierte Position nicht eine Nivellierung der gesamten Lebensbedingungen aller Pflegebedürftigen.

${ }^{57}$ Es sei nochmals betont, daß diese Variante der Zielbestimmung als eine mögliche Denkrichtung durchgespielt wird; nicht mehr und nicht weniger.
} 
unerwünschter Effekt kann zumindest teilweise vermieden werden, wenn die Bemessung des Selbstbehalts auf die individuelle Zahlungsfähigkeit Rücksicht nimmt. Prinzipiell unbedenklich bleibt die oben abgegrenzte "unechte" Selbstbeteiligung, die sich allein nach der mit Pflegebedürftigkeit evtl. verbundenen individuellen Ersparnis in der Lebensführung bemißt.

ad 3. (Leistungsform): Der Anspruch gleichen Zugangs aller zu Pflegediensten verlangt zwingend die Einrichtung eines Sachleistungsverfahrens. ${ }^{58}$ Bei Auszahlung von Pflegegeldern bleiben die Wohlstandsdiskrepanzen zwischen den Bedürftigen (auf absolut höherem Niveau) erhalten. Da nicht ausgeschlossen werden kann, daß die Transferleistung entweder (teilweise) anderweitig verwendet oder umgekehrt aus eigenen Mitteln zum Kauf von Pflegeleistungen aufgestockt wird, werden die unterschiedlichen Zahlungsfähigkeiten bei gleicher Intensität des Bedürfnisses zu unterschiedlichen Zahlungsbereitschaften führen. Selbst wenn genügend Kapazitäten bereitstehen, um eine Versorgung aller Bedürftigen zu gewährleisten, werden sich vermutlich auch im Kernbereich der Pflege ${ }^{59}$ unterschiedliche Güteklassen herausbilden; die Wahlmöglichkeiten der besser Situierten bei der Befriedigung ihres Bedarfs sind dann c.p. größer als die der "Armen".

Dies muß freilich dann noch nicht als Verletzung des Gleichheitspostulats angesehen werden, wenn sich diese erweiterten Optionen allein auf "Luxus-Pflege" beziehen, auf die ein Grundrecht nicht besteht. ${ }^{60}$ Aber selbst wenn die Geldleistungen so bemessen und an die jeweilige Preisentwicklung angepaßt werden, daß sie - ohne Selbstbeteiligung - zum Erwerb aller jeweils als erforderlich angesehenen Leistungen ausreichen, ist ein "gleicher Zugang" im strengen Sinn insoweit nicht gegeben, als der Anreiz zur Zweckentfremdung von Pflegegeld bei geringem sonstigem Einkommen c.p. größer ist als bei hohem. Diese Überlegung zeigt, daß der hier vorgetragene Ansatz stark meritorische Züge trägt.

\footnotetext{
58 vgl. L.C. Thurow (1974)

${ }^{59}$ Im Bereich von Nebenleistungen (etwa der besonders bevorzugten Lage eines Pflegeheims) können Qualitätsunterschiede als unbedenklich gelten.

${ }^{60}$ Nochmals: Die Grenze zwischen Bedarf und Luxus ist höchst unklar und kann konkret nur politischgesellschaftlich erfolgen. Prinzipiell aber gehört zum unabweisbaren Bedarf im Sinne der hier verfolgten Argumentation all das, was im Rahmen des Sinnvollen und Möglichen dazu dient, den Nachteil des Pflegefalls gegenüber dem "normalen", davon unbelasteten Leben auszugleichen.
} 
Im Extremfall muß die Wahrung des Grundrechts selbst gegen die geäußerten Präferenzen seiner Inhaber durchgesetzt werden. ${ }^{61}$ Anders gesagt: Das Ziel, den von Krankheit und ähnlichen Nachteilen Betroffenen durch Heilung und Betreuung möglichst "normale" Wahlmöglichkeiten zu eröffnen, kann in seiner Durchsetzung auch mit der Einschränkung von Wahlfreiheit an anderer Stelle verbunden sein.

Diesem etwas wunden Punkt entspricht auch die von DANIELS vorgenommene, implizit hierarchiche Trennung zwischen (objektiven) "needs" und "desires". ${ }^{62}$ Eine solche Unterscheidung wird nicht nur mit Zuordnungsschwierigkeiten im Einzelfall zu kämpfen haben, sondern ist auch im Grundsdatz kritisierbar. DANIELS räumt demgemäß ein, keine "Letztbegründung" für sein Konzept liefern zu können. ${ }^{63}$ Hilfsweise beruft er sich daher auch auf die allgemeine moralische Praxis, die eine Differenzierung zwischen medizinisch begründetem Bedarf und sonstigen Wünschen vornimmt. ${ }^{64}$ Analog dazu geht es auch THUROW nicht darum, eine Sonderstellung medizinischer Leistungen aus moralischen Gründen zu deklarieren, sondern ethisch motivierte Vorstellungen aufzufinden, mit denen sich die weithin beobachtbare Aussonderung der Gesundheitsversorgung aus dem Marktgeschehen erklären läßt. ${ }^{65}$

\footnotetext{
${ }^{61}$ Es sei allerdings an das oben angeführte Argument erinnert, daß mitunter Grund zu der Befürchtung besteht, daß die Zweckentfremdung von anderen gegen die Wünsche des Empfängers selbst durchgesetzt wird.

${ }^{62}$ siehe N. Daniels (1981), S. 152

${ }^{63}$ Dies giltselbstverständlich auch für das konkurrierende Konzept der grundsätzlichen Markttauglichkeit von Gesundheitsgütern bzw. Krankenversicherungen.

64 "If I appeal to my friend's duty of beneficence in requesting $\$ 100$, I will most likely get a quite different reaction if I tell him I need the money to get a root-canal than if I tell him I need the money to go to the Brooklyn neighborhood of my childhood to smell pickles in a barrel." Daniels (1981), S. 151

${ }^{65}$ L.C. Thurow (1977), S. 192
} 


\section{Effizienzkriterien}

\subsection{Einleitung}

Nachdem das Ziel einer Absicherung gegen die Folgen des Pflegefalls in seinen verschiedenen Komponenten bestimmt worden ist, muß nunmehr geklärt werden, unter welchen Bedingungen ein System zur Erfüllung dieser Vorgaben als effizient gelten kann, indem es vermeidbare Wohlfahrtsverluste vermeidet. Eine solche Prüfung betrifft zum einen die Finanzierung, zum andern das Leistungswesen. In der ersten dieser beiden Sphären lassen sich wiederum zwei wesentliche Kriterien benennen:

Im Hinblick auf die Finanzierung ist zunächst zu fordern, daß sie auch auf lange Sicht verläßlich ist. Die Erfüllung der Schutzziele muß auch für diejenigen Teilnehmer am Verfahren gewährleistet sein, bei denen der eventuelle Eintritt des Pflegefalls erst in fernerer Zukunft zu erwarten ist. Andernfalls werden sie die ihnen abgeforderten Finanzierungsbeiträge mit schädlichen Folgen für das System zu verweigern trachten ${ }^{1}$ bzw. als Wohlfahrtseinbuße erfahren.

So gesehen ${ }^{2}$ stellt das Verläßlichkeitspostulat bereits eine Ausprägung des Äquivalenzgedankens dar, der auch dem zweiten Aspekt der Finanzierungseffizienz zugrundeliegt. Die Höhe der einzelnen Beiträge soll an der jeweiligen Leistungserwartung ausgerichtet sein. Im Idealfall wird damit erreicht, daß die Einführung der Versicherung eine Pareto-Verbesserung darstellt: Wenn ein jeder nach Maßgabe dessen zu zahlen hat, was er an Gegenleistung erwarten $\mathrm{kann}^{3}$, erleidet niemand eine Verschlechterung seiner Nutzenposition. Anhand dieser Maßstäbe wird zunächst den Fragen der Finanzie-

\footnotetext{
${ }^{1}$ Etwa so argumentiert Thiede (1990), S. 144f., wenn er die "Akzeptanz" der Pflegeversicherung als Effizienzkriterium bespricht. Der dort behauptete enge Zusammenhang zwischen Akzeptanz und Beitragsmoral ist nicht unplausibel, aber theoretisch dennoch zweifelhaft. Ein Free-Rider-Verhalten ist ökonomisch auch für solche Individuen reizvoll, die dem System als solchem positiv gegenüberstehen.

${ }^{2}$ Alternativ könnte die Verläßlichkeit auch als eine den Aspekten des Leistungsinhalts übergeordnete Zielkomponenteangesehen werden. Wegen ihres Bezugs zur Finanzierungsfrage, die im vorigen Kapitel noch keine Rolle gespielt hat, erscheint es aber zweckmäßiger, sie in den Effizienzzusammenhang zu stellen. Gleichwohl ist die ungenügende Erfüllung dieses Kriteriums letztlich auch zielschädlich.

${ }^{3}$ Unerheblich ist dabei, daß die Versicherung in einer ex-post-Betrachtung für all all diejenigen einen Vermögensnachteil bewirkt, denen der Schadensfall erspart bleibt. Vgl. dazu etwa Wolfgang Müller (1988), S.141, der klarstellt, daß die eigentliche Leistung der Versicherung nicht in der Zahlung im Schadensfall, sondern in deren bedingter Zusage liegt.
} 
rungsquelle, der Beitragsbemessung und des Finanzierungsverfahrens nachgegangen (Teil 4.2).

Auf der Leistungsseite konzentriert sich die Betrachtung auf den "Moral hazard" im Sinne der möglichen effizienzschädlichen Folgen von Verhaltensänderungen, die eine Pflegeversicherung auf seiten der Versicherten bewirkt. Da diese Untersuchung breiten Raum einnimmt, unterbleibt die Ausarbeitung eines Kriteriums in bezug auf den Wettbewerb zwischen den Anbietern von Pflegeleistungen bzw. Versicherungsschutz, obwohl auch dieser Aspekt für die Effizienz von Sicherungssystemen fraglos von Bedeutung ist. Auf seine Behandlung an dieser Stelle kann dennoch verzichtet werden, da die entsprechende Diskussion - anders als die um den Moral hazard - im Fall eines Sachleistungssystems wenig pflegespezifisch wäre, sondern weitgehend identisch mit derjenigen, die in Deutschland über den "Kassenwettbewerb" in der GKV geführt wird. Insofern kann auf die einschlägige Literatur ${ }^{4}$ und im übrigen darauf verwiesen werden, daß im folgenden Kapitel ${ }^{5}$ ein Überblick über die entsprechende Reformdebatte in den Niederlanden gegeben wird.

Dort wird sich zeigen, daß das Ergebnis der in den Niederlanden eingeleiteten Veränderungsprozesse in Richtung auf mehr Wettbewerb im Gesundheitswesen im allgemeinen und bzgl. der Pflege im besonderen derzeit völlig offen ist, so daß eine Bewertung der niederländischen Pflegeversicherung in Hinsicht auf diesen Aspekt ohnehin kaum möglich bzw. sinnvoll wäre.

\subsection{Effizienz in Hinblick auf die Finanzierung der Pflegesicherung}

\subsubsection{Das Kriterium der Verläßlichkeit und seine Folgen für die Wahl der Finan- zierungsquelle}

Trivialerweise kann die Existenz einer Institution zum Schutz vor den Folgen der Pflegebedürftigkeit nur insoweit einen versicherungstypischen Nutzen stiften, wie die in Aussicht gestellten Leistungen auch wirklich als gesichert empfunden werden. Wenn dies nur eingeschränkt der Fall ist, droht die zwangsweise Erhebung von Finanzierungsbeiträgen einer Schlechterstellung der Versicherten gleichzukommen und somit

\footnotetext{
${ }^{4}$ siehe beispielsweise K. Jacobs (1993) oder C. Felkner/P. Stein (1992)

${ }^{5}$ siehe Abschnitt 5.5.1
} 
effizienzschädlich zu wirken. Der für die Leistungserwartung relevante Zeithorizont reicht dabei bis zum spätestmöglichen Eintrittszeitpunkt des individuellen Pflegerisikos. Die diesbezüglichen Erwartungen werden sich - abgesehen von subjektiven Einschätzungsmustern - anhand der über die zukünftige Entwicklung verfügbaren Informationen sowie aufgrund der Erfahrungen bilden, die in Hinblick auf die Stabilitätsmerkmale des betreffenden Systems bereits gesammelt werden konnten. Je unvollständiger die ersteren sind, desto maßgeblicher werden die letzteren.

Somit läßt sich sagen, daß jede Verschlechterung der Leistungskonditionen nicht nur direkt zu einer verminderten Zielerreichung im oben umrissenen Sinn führt, sondern darüber hinaus die Erwartungen beschädigt, deren Erhaltung auch selbst als Teil des Sicherungsziels betrachtet werden kann. Weitere Einschränkungen des Leistungsversprechens werden dann als Befürchtungen antizipiert. Die Formen, in denen derartige Leistungsminderungen auftreten können, lassen sich entlang der in Kapitel 3 aufgeführten Zieldimensionen unterscheiden, wobei der erste Punkt, nämlich Änderungen im Zuschnitt des gesicherten Personenkreises, als unwahrscheinlich ausgeschlossen bleibt:

- $\quad$ Einschränkungen beim Leistungszugang: Eine Verengung des Kreises der Leistungsempfänger muß nicht unbedingt über entsprechende Veränderungen in der Definition der Anspruchsvoraussetzungen erfolgen, sondern stellt sich möglicherweise auch dann ein, wenn die Leistungskapazitäten - etwa die Zahl der Heimpflegeplätze - im Verhältnis zum jeweils definierten Bedarf zu knapp gehalten werden. Wenn dies nicht zu einer durchweg restriktiveren Anerkennungspraxis mit der Folge eines Ausschlusses leicht Pflegebedürftiger führt, können auch unsystematisch verteilte Leistungslücken und/oder Wartelisten entstehen.

- Abbau des finanziellen Schutzes im Sinne erhöhter Selbstbeteiligung: Auch hier sind zwei Fälle zu unterscheiden. Existieren numerisch fixierte Regeln zum Selbstbehalt, so können diese verändert werden. Ergibt sich die Selbstbeteiligung hingegen residual aus monetär bestimmten Höchstgrenzen der Versicherungsleistung ${ }^{6}$, so führt bereits der Verzicht auf die Anpassung dieser Grenzen an steigende Preise für Pflegeleistungen zu einer allmählichen Aushöhlung der Schutzwirkung.

\footnotetext{
${ }^{6}$ Dies gilt für das deutsche Pflegeversicherungsgesetz, siehe Kapitel 7.
} 
- $\quad$ Einschränkungen im Leistungskatalog: Solche Verschlechterungen sind dann möglich, wenn das System Sachleistungen gewährt. Auch hier läßt sich eine "passive" Variante denken, wenn nämlich innovative Leistungen, die in Reaktion auf neuartige Bedarfsprofile oder aufgrund geriatrischer Erkenntnisfortschritte aufkommen und bereits als fortschrittlich im Sinne der Pflegeziele erwiesen sind, nicht in den Leistungskatalog aufgenommen werden. Auch dies stellt eine relative Leistungseinschränkung dar, wenn die Schutzzusage auf Pflegeleistungen nach dem jeweiligen "Stand medizinisch-pflegerischer Erkenntnisse"7 lautet.

- Verminderung von Pflegequalität: Worin eine solche bestehen kann, ergibt sich aus den Ausführungen zum Qualitätsaspekt. Es ist dort auch schon darauf hingewiesen worden, unter welchen Bedingungen die Beibehaltung eines gegebenen Leistungsstandards eine Verbesserung des Personalschlüssels erfordert. In solchen Fällen kann also auch hinsichtlich der Qualität ein effektiver Leistungsabbau als Folge bloßer Unterlassungen eintreten.

Es stellt sich nun die Frage, inwieweit sich aus dem Verläßlichkeitsgebot im Sinne der Vermeidung solcher Einschnitte konkrete Anforderungen an die Gestaltung des Sicherungssystems ableiten lassen. Zu denken ist dabei zum einen an eine entsprechende Formulierung des Leistungsversprechens, wie sie sich direkt aus den skizzierten Aushöhlungsgefahren ergibt. Ebenso wichtig ist aber die juristische Natur der Ansprüche und ihr institutioneller Hintergrund. Auch die umfassendste Leistungszusage ist dann prekär, wenn sie im Grundsatz jederzeit revidiert werden kann. Unter diesem Aspekt soll nunmehr eine erste Kritik der Finanzierungsverfahren erfolgen, die für eine Absicherung des Pflegerisikos in Betracht kommen.

Grundsätzlich kann die Finanzierung von Pflegeleistungen, soweit sie im Sinne des Sicherungsziels nicht von den Betroffenen selbst bestritten werden soll, aus drei Quellen erfolgen: aus allgemeinen Steuermitteln, aus Sozialversicherungsbeiträgen oder aus Versicherungsprämien. Die letzten beiden Varianten seien dabei definitorisch so unterschieden, daß die Beiträge in Relation zum Einkommen bemessen werden, während die Prämien davon unabhängig sind. Damit bleiben beide Typen grundsätzlich offen für verschiedene Tarifgestaltungen im einzelnen.

\footnotetext{
${ }^{7}$ Auch diese Formulierung entstammt dem deutschen PflegeVG (§ 69).
} 
Zunächst sei hier auf die Möglichkeit der Steuerfinanzierung eingegangen. Unter dem Titel "Leistungsgesetz" ist dieser Weg zeitweilig von verschiedenen Seiten vorgeschlagen worden. Sowohl die GRÜNEN im Bundestag, als auch das Land Rheinland-Pfalz sowie die Deutsche Angestellten Gewerkschaft im Verein mit Ärzteverbänden haben bis 1990 entsprechende Konzepte vorgelegt. ${ }^{8}$ Von Seiten der politischen Linken ist dabei insbesondere die insgesamt "gerechtere", weil an der Leistungsfähigkeit der einzelnen orientierte Lastenverteilung als Vorzug herausgestrichen worden, der mit einem solchen Konzept verbunden sei. ${ }^{9}$

Als Erklärung dafür, daß dieser Lösungsweg mittlerweile an Anziehungskraft verloren zu haben scheint und in der Debatte um verschiedenartige Versicherungslösungen weitgehend untergegangen ist ${ }^{10}$, wird auf die angespannte Lage der öffentlichen Haushalte insbesondere infolge der deutschen Vereinigung verwiesen. ${ }^{11}$ Dies leuchtet auf den ersten Blick nicht recht ein, da ja die alternativen Vorschläge ebenfalls mit zusätzlichen Belastungen für die privaten Haushalte verbunden sind, die prinzipiell ohne weiteres durch eine Steuererhöhung im Umfang des pflegebedingten Mittelbedarfs substituiert werden könnten.

Sinn hat ein solches Argument daher nur in Verbindung mit der Implikation, daß pflegespezifische Lasten in Form von Beiträgen oder Prämien c.p. leichter hinnehmbar seien als zusätzliche Steuern. Ob dies zutrifft, ist zum einen eine empirische Frage an die "Steuerwiderstandsforschung" ${ }^{12}$, der an dieser Stelle nicht nachgegangen wird. Entscheidend ist hier vielmehr der wesentliche Grund für die Annahme eines solchen qualitativen Unterschiedes: Im Fall von Steuern besteht kein Anspruch auf spezifische staatliche Gegenleistung. ${ }^{13}$ Alle staatlichen Ausgaben sind im Grundsatz disponibel;

\footnotetext{
${ }^{8}$ Eine detaillierte Synopse findet sich bei D. Seiler (1991), S. 351.

${ }^{9}$ Siehe etwa den Beitrag des Abgeordneten Gysi in: Das Parlament (1993), S. 7: "Es gibt nur eine wirklich gerechte Lösung. Das ist die Steuerfinanzierung". Ähnlich war die Haltung linker Parteien in den Niederlanden in der dortigen Debatte um die Pflegeversicherung (siehe Kap. 5.1).

${ }^{10}$ Gelegentlich tauchen entsprechende Vorschläge indes noch auf. Siehe etwa R. Eisen (1992), S. 240 oder die Äußerungen des DAG-Funktionärs Quartier im Handelsblatt vom 23.9.1991 unter dem Titel "Leistungsgesetz als billiger und gerechter bezeichnet."

${ }^{11}$ vgl. D. Seiler (1991), S. 350

${ }^{12}$ siehe dazu K. Mackscheidt (1985), der seine These von der größeren "Verträglichkeit" von Sozialversicherungsbeiträgen gegenüber Steuern allerdings vorwiegend auf Plausibiliätserwägungen stützt.

${ }^{13}$ siehe Abgabenordnung, $\$ 3$ (1)
} 
sie können und sollen im politisch determinierten Prozeß der Haushaltsaufstellung gegeneinander abgewogen und verschoben werden. Auch für den Pflegebereich gilt damit in jedem Haushaltsjahr aufs neue: "Der Finanzierer [und dies ist der Staat! S.W.] bestimmt Umfang, Art und Qualität der Leistungen."14

Was EISEN so als Vorteil eines Leistungsgesetzes im Sinne der Kostenkontrolle geltend macht, führt im Rahmen des hier verfolgten Ansatzes zum Ausschluß der steuerfinanzierten Variante von der weiteren Betrachtung: Wenn der Staat in prinzipiell freier Entscheidung und im Lichte der jeweiligen Haushaltslage über die Leistungsgewährung verfügt, so besteht von vornherein keine auf Dauer verbindliche Leistungszusage und damit auch kein echter Schutz. Versicherungsbeiträge hingegen sind - auch im Fall der Sozialversicherung - "grundsätzlich aus der laufenden Auseinandersetzung um Prioritäten in den öffentlichen Haushalten herausgehalten" und die daraus finanzierten Aufgaben deshalb "weniger in der Gefahr, zurückgedrängt, vernachlässigt, nicht mehr erfüllt zu werden." ${ }^{15}$ Insbesondere wäre davon auszugehen, daß eine solche Lösung sich bzgl. des Leistungsumfangs in Richtung einer gesonderten Mindestsicherung entwickeln würde ${ }^{16}$, die analog zur allgemeinen Sozialhilfe erst dann eintritt, wenn die eigenen Mittel des Betroffenen erschöpft sind.

Angesichts der Vielzahl und Wichtigkeit alternativer Verwendungszwecke für Steuergelder ist es auf Dauer politisch kaum durchsetzbar, Pflegehilfe auch den "Reichen" zu gewähren. Deswegen sind haushaltsfinanzierte Sozialleistungen, die ohne jede Prüfung der finanziellen Bedürftigkeit gewährt werden, auch recht selten und werden diese Voraussage sei im Lichte aktueller Diskussionen etwa um das deutsche Kindergeld gewagt - in Zukunft noch seltener sein. ${ }^{17}$ Eine Pflegesicherung "nur für Arme" genügt dem hier verfolgten und begründeten Anspruch einer auf das Risiko Pflegefall

\footnotetext{
${ }^{14}$ R. Eisen (1992), S. 241

${ }^{15}$ D. Schäfer (1982), S. 333

${ }^{16}$ Wenn sie nicht schon von vornherein darauf angelegt wäre, wie dies z.B. beim Vorschlag Quartiers (siehe Fußnote 10) der Fall ist.

${ }^{17}$ Für K.-D. Henke (1990), S. 15, ist es selbstverständlich, daß "im Rahmen sog. Leistungsgesetze Bedürftigkeitsprüfungen erforderlich (werden), die nur schwer vom Odium der Sozialhilfe zu befreien sein werden".
} 
bezogenen Schutzvorrichtung nicht. Ein aus Steuern finanziertes Leistungsgesetz ist damit schon im Ansatz weder zielgerecht, noch effizient. ${ }^{18}$

\subsubsection{Das Äquivalenzkriterium und seine Folgen für die Bewertung des einkommensabhängigen Beitrags}

\subsubsection{Der Grundgedanke: Mindestsicherung und Beitragsäquivalenz}

Die Betrachtung beschränkt sich nunmehr auf die Versicherungslösungen und damit zunächst auf die Frage, ob unter Effizienzgesichtspunkten ein Zusammenhang zwischen dem Einkommen der Versicherten und den auf sie entfallenden Beiträgen bzw. Prämien wünschenswert ist oder nicht. Im Sinne der oben vorgenommenen Definition bleibt dabei noch offen, ob neben dem Einkommen oder an dessen Statt andere Faktoren die Höhe des jeweiligen Beitrags beeinflussen.

Vorab gilt es nunmehr das Effizienzziel im hier relevanten Sinn zu bestimmen. Dies kann wiederum mithilfe des Pareto-Kriteriums geschehen: Eine Regelung zum Schutz vor den Folgen der Pflegebedürftigkeit sollte möglichst so beschaffen sein, daß sie im Vergleich zur Ausgangslage zumindest einige Individuen besser und niemanden schlechter stellt. Eine Lösung, welche diese Bedingung erfüllt, ist solchen Alternativen vorzuziehen, von denen dies nicht behauptet werden kann. ${ }^{19}$ Weiter oben ist dargelegt worden, daß und aus welchen Gründen eine von spezifischen Staatseingriffen im

\footnotetext{
${ }^{18}$ Eine empirische Ausfüllung dieser generellen Feststellung ist hier nicht am Platze. Es würde sich ohne weiteres belegen lassen, daß gerade gesetzlich geregelte Sozialleistungen im Falle von Haushaltsengpässen regelmäßig als Träger von Sparpotential zur Debatte stehen. Dann aber müßte freilich mit in Betracht gezogen werden, daß auch Sozialversicherungen gegen haushaltspolitisch motivierte Zugriffe des Staates nicht vollständig immun sind, so daß sich der grundsätzliche Unterschied auf praktischer Ebene verwischt. Er bleibt aber darin bestehen, daß Staatszugriffe auf die Beitragsmittel der Sozialversicherung immer nur indirekt erfolgen und - jedenfalls nach deutschem Recht - in der auch für gezahlte Beiträge geltenden Eigentumsgarantie ihre Grenze finden (vgl. R. Eisen (1992), S. 238 und die dort genannten Urteile des BVerfG.) Wo diese Grenze genau verläuft, ist allerdings umstritten. Dies hat sich z.B. im Jahre 1993 in der Diskussion um Kürzungen der Lohnersatzleistung bei Arbeitslosigkeit gezeigt (vgl. C. Ullmann (1993)).

${ }^{19} \mathrm{~J}$. Hackmann (1978) bestreitet die Zulässigkeit ebendieser Empfehlung, da sich eine so als unterlegen aussortierte Lōsung bei Bekanntwerden der sozialen Wohlfahrtsfunktion doch als die bessere herausstellen könne. Dieses Argument erscheint mir nicht stichhaltig, da das Pareto-Kriterium seine Berechtigung, ja seine Existenz erst aus der Tatsache bezieht, daß die soziale Wohlfahrtsfunktion nach herrschender Meinung eben nicht ermittelt werden kann.
} 
Bereich der Pflegesicherung freie Situation Spielräume für derartige Wohlfahrtsverbesserungen birgt: Eine Nachfrage nach Sicherheit, deren Vorhandensein aufgrund einer generellen Risikoabneigung unterstellt werden kann, stößt bei der Umsetzung in Marktnachfrage und bei deren Befriedigung auf Hindernisse.

Eine idealtypische Versicherung wird dem Pareto-Kriterium im Grundsatz jedenfalls dann genügen, wenn das Äquivalenzprinzip gilt, d.h. wenn der "nach risikotheoretischen Grundsätzen kalkulierte Erwartungswert der Versicherungsleistung gleich (...) dem Erwartungswert der Beitragszahlung ist. ${ }^{120}$ Ein bereits im zweiten Kapitel besprochener Hinderungsgrund verdient dabei aber wiederum besondere Beachtung: die Existenz einer wie auch immer ausgestalteten staatlichen Mindestsicherung, auf die zur Befriedigung unabweisbarer Grundbedürfnisse zurückgegriffen werden kann, wenn und sofern die jeweils eigenen Mittel dazu nicht ausreichen. Daß eine solche Vorrichtung besteht, kann zumindest für Industriegesellschaften ohne genauere Prüfung theoretischer Begründungsmuster und historischer Zusammenhänge ${ }^{21}$ als fraglos gegeben betrachtet werden. Sie stellt ein fundamentales zivilisatorisches Element staatlich verfaßter Gesellschaften dar. Ebenfalls unstrittig ist, daß Pflegebedürfnisse grundsätzlich - also ohne Ansehen eventueller Abgrenzungsschwierigkeiten - aufgrund ihres existenziellen Charakters in den Bereich gehören, den die Mindestsicherung notfalls abdecken muß.

Es ist gezeigt worden, daß die Gewißheit, solchen Schutz im Bedarfsfall in Anspruch nehmen zu können, die Marktnachfrage nach Pflegeversicherungen hemmt. Hieraus kann freilich nicht gefolgert werden, daß sie das entsprechende Schutzbedürfnis befriedigt: Zwar ist unter allen Umständen eine (an Mindeststandards orientierte) Versorgung gewährleistet; die Mindestsicherung greift aber ihrer Natur nach erst dann, wenn die eigenen finanziellen Möglichkeiten - evtl. einschließlich von Unterhaltsansprüchen, die gegen Angehörige geltend gemacht werden können - restlos erschöpft sind. Einkommen und Vermögen bleiben somit ungeschützt.

\footnotetext{
${ }^{20}$ G. Heubeck (1982), S. 304

${ }^{21}$ Erste städtische Armenordnungen, die eine Gewährung öffentlicher Unterstützung nach entsprechender Prüfung der Bedürftigkeit vorsahen, gehen im Bereich des heutigen Deutschland auf das Jahr 1522 zurück und sind damit weit älter als jegliche andere Form von Sozialpolitik (vgl. J. Frerich (1990), S. 115).
} 
Damit aber verschiebt sich gegenüber einer Situation ohne Mindestsicherung der für den einzelnen Versicherungsnehmer mit der Pflegeversicherung verbundene Schutzeffekt: Der sonst entscheidende "Erwartungsschaden" als Produkt aus der Wahrscheinlichkeit und den durchschnittlich anzunehmenden Kosten des Pflegefalls muß korrigiert werden um den Betrag, den der Betroffene aus Mitteln der Mindestsicherung ohnehin erwarten kann. Dieser Korrekturfaktor aber verhält sich umgekehrt proportional zum Einkommen bzw. Vermögen: Je mehr jemand an Eigenmitteln zu verlieren hat, desto weniger (bzw. desto später im Verlauf der Bedürftigkeit) profitiert er von der Mindestsicherung.

Diese Feststellung hat unter der Zielvorgabe einer mit der Versicherung erstrebten Pareto-Verbesserung Implikationen für die Beitragsgestaltung. Da die Gefahr, pflegebedürftig zu werden, aus individueller Sicht ein finanzielles Großrisiko darstellt, kann den Einzelnen ein Interesse daran unterstellt werden, dieses Risiko auszuschalten. Entsprechen sich in idealer Weise die Prämienzahlungen und die Leistungserwartung, herrscht also individuelle Beitragsäquivalenz, so erfolgt durch den Abschluß der Versicherung eine Besserstellung. ${ }^{22}$ Unterscheiden sich Beiträge und Leistungen aufgrund von Verwaltungskosten und/oder Gewinnen der Versicherung, so gilt dies nur noch unter der Voraussetzung, daß die Risikoaversion hinreichend groß ist. Aber auch dann ist eine Besserstellung aller durch die Versicherung umso wahrscheinlicher, je genauer die Beitragsbemessung dem Äquivalenzprinzip folgt.

Die Leistungserwartung, an der sich die Beiträge demnach ausrichten sollten, ist der Erwartungswert des Schadens abzüglich des Erwartungswertes für die Transferzahlungen aus der Mindestsicherung. Da die letzteren durch das Einkommen bestimmt werden, ist eine Einkommensabhängigkeit der Beiträge aus allokativen Gründen zu befürworten. ${ }^{23}$ Dies soll im folgenden spezifiziert werden.

\subsubsection{Die Einkommensabhängigkeit des Beitrags - eine Spezifikation}

Im folgenden soll die allokative Begründung für die Einkommensabhängigkeit des Beitrags in ihren Konsequenzen näher bestimmt werden. Zunächst ist die Schadenshöhe

\footnotetext{
22 vgl. M Nell (1992) S. 45f.

${ }^{23}$ W. Strassl (1988), insbes. S. 232ff., entfaltet diesen Gedanken ausführlich für die gesetzliche Krankenversicherung.
} 
im Einzelfall bei gegebenen Pflegepreisen abhängig von der Dauer und dem über die Dauer im Durchschnitt gegebenen Ausmaß der Pflegebedürftigkeit. Diese Aussage birgt die wichtige Implikation eines "konstanten Versorgungsniveaus" in dem Sinne, $\mathrm{da} ß$ sich einer bestimmten Pflegebedürftigkeit bestimmte Pflegeleistungen quasi objektiv zuordnen ließen. Ausgeblendet ist damit nicht allein die Problematik des Moral hazard $^{24}$, sondern auch die mögliche Abhängigkeit der Leistungsstandards vom Finanzierungssystem. Wenn also mit der Einführung einer Pflegeversicherung auch eine generelle Anhebung der Versorgungsdichte und/oder der Qualität von Pflege intendiert ist, so bleibt dies für die in diesem Teilkapitel verfolgte Argumentation außer Betracht. Der Sinn der Versicherung verkürzt sich damit auf den Aspekt der Einkommenssicherung im Pflegefall.

Da sich die Untersuchung auf die Frage konzentriert, ob eine Einkommensabhängigkeit des Beitrags zu befürworten ist, bleiben andere für das Risiko relevante Merkmale weitgehend ausgeklammert. Es kann somit nicht der Anspruch erhoben werden, daß eine insgesamt optimale Beitragsgestaltung abgeleitet würde. Zur Rechtfertigung und Abrundung dieses selektiven Vorgehens werden wesentliche vernachlässigte Faktoren aber im Vorwege kurz inspiziert:

An erster Stelle ist das Geschlecht zu nennen, das die Schadenserwartung in bezug auf den Pflegefall stark beeinflußt. ${ }^{25}$ Es ist eine "eigenständige verteilungspolitische Entscheidung" 26 , ob dieser Faktor bei der Beitragsbemessung ignoriert werden soll, was - für sich betrachtet - selbstverständlich einer Umverteilung zulasten der Männer gleichkommt. Weiterhin spielt das bereits erreichte Lebensalter eine große Rolle, wenn die Versicherung zu einem bestimmten Zeitpunkt für alle eingeführt wird. Hierauf wird weiter unten eingegangen.

Weitere mögliche Risikofaktoren sind in ihrer Relevanz zweifelhaft: Zum einen ist denkbar, daß das jeweils vorhandene und einsatzbereite private Pflegepotential einen

\footnotetext{
${ }^{24}$ Sie wird im Abschnitt 4.3 gesondert behandelt.

${ }^{25}$ Lt. Infratest (1992), Tabelle 1, sind von den in Privathaushalten in Deutschland (West) lebenden älteren Personen mit regelmäßigem Pflegebedarf $70 \%$ weiblich. Die Bewohnerschaft der Pflegeheime (ohne unter 65jährige) besteht nach W. Krug/G. Reh (1992), S. 29, sogar zu über $83 \%$ aus Frauen. Nach P. Zweifel et al. (1994), S. 66, liegt dies - zumindest in der Schweiz - ausschließlich an der für Frauen deutlich höheren Lebenserwartung.
}

${ }^{26}$ G. Rolf, P.B. Spahn, G. Wagner (1988), S. 27 
Einfluß auf die bei der Versicherung anfallenden Kosten haben kann. Bei diesem Faktor handelt es sich aber um eine sehr diffuse Größe, die vom Versicherer nicht zuverlässig erfaßt werden kann und deren Entwicklung auch für den Versicherten selbst über längere Zeiträume nicht absehbar ist. Die von THIEDE vorgeschlagene Approximation über Familienstand und Kinderzahl erscheint - zumal unter heutigen Bedingungen gestiegener Flexibilität, Vielfalt und Unverbindlichkeit familiärer und anderer Beziehungen inadäquat: ${ }^{27}$ Weder sind (enge) Verwandte per se als potentielle Pflegepersonen anzusehen, noch müssen zur Pflege bereite nahestehende Menschen Verwandte sein. Zudem ist der Familienstand veränderlich.

Weiterhin ist nicht auszuschließen, daß sich auch andere Gruppen erhöhten Risikos identifizieren lassen. $\mathrm{Zu}$ denken ist dabei aber wohl allenfalls an solche Personen, die an bestimmten chronischen Krankheiten bereits leiden. ${ }^{28}$ Für die Krankenversicherung häufig diskutierte Faktoren der Lebensführung (Genußmittelkonsum, Übergewicht) kommen kaum in Betracht, da ihr Einfluß auf das Pflegerisiko uneindeutig ist.

Um nun den über die Mindestsicherung vermittelten Einfluß des Einkommens auf den tatsächlichen Erwartungswert der Pflegeversicherungsleistung klar herausarbeiten zu können, sind einige vereinfachende Annahmen zu treffen. Erstens wird die mögliche Existenz von Vermögen nicht eigens beachtet, obwohl auch ein solches im unversicherten Pflegefall hingegeben werden müßte, bevor die Mindestsicherung in Anspruch genommen werden kann. Diese Vereinfachung erscheint vertretbar, da der Versicherungsnehmer zum Zeitpunkt seines Beitritts in der Regel kaum absehen kann,

\footnotetext{
${ }^{27}$ vgl. R. Thiede (1990), S. 200ff. Der Autor hebt zunächst (S. 186ff.) ausführlich die kaum überschaubare Komplexität des Merkmals "Umfang der personellen häuslichen Pflegeressourcen" hervor, um es sodann für die Beitragsstaffelung unter Hinweis auf die andernfalls eintretenden Erfassungskosten und -probleme auf die beiden genannten Indikatoren zu verkürzen. Die Fragwürdigkeit dieses Vorgehens wird schon daran deutlich, wie unpassend hinterher die Benennung des Kriteriums erscheint: "Häusliche Ressourcen" werden nach Personen bemessen, deren faktischer Wohnort unberücksichtigt bleibt.

${ }^{28}$ Die These von P. Zweifel et al. (1994), S. 67, "die Wahrscheinlichkeit, ein Pflegefall zu werden, hängt vor allem vom Gesundheitszustand ab", ist in hohem Maße irreführend. Dort ist lediglich geprüft worden, ob sich die heutigen Heimbewohner von den übrigen Mitgliedern der Stichprobe in Hinblick auf ihren Gesundheitszustand vor vier Jahren unterscheiden. Gezeigt wird folglich allenfalls, daß dem Eintritt der Pflegebedürftigkeit oftmals eine Phase schwacher Gesundheit vorausgeht. Daß von den "vor vier Jahren Kranken" viele schon gestorben sein dürften, bleibt ebenso unerfaßt wie die Tatsache, daß von den "vor vier Jahren Gesunden" manche in dreißig Jahren noch leben - und zwar im Pflegeheim.
} 
ob und in welcher Höhe er im Alter ein solches Vermögen aufgebaut haben wird. Schätzen kann er es allenfalls aufgrund seines Einkommens und einer Prognose seiner Sparneigung. Auch diese aber unterliegt im Lebenslauf dem Einfluß verschiedenartiger äußerer und subjektiver Faktoren und ist daher nur sehr eingeschränkt im voraus kalkulierbar. Da auch Erwartungen bezüglich exogener Vermögenszugänge wie Erbschaften über lange Zeiträume mit großer Unsicherheit behaftet sind, kann der erwartete Vermögensumfang im wesentlichen als eine Funktion des Einkommens aufgefaßt und daher vernachlässigt werden.

Zum zweiten sei zunächst unterstellt, daß die Pflegebedürftigkeit im Fall ihres Eintritts pro Periode Kosten in einer für alle Betroffenen einheitlichen Höhe $(\mathrm{P})$ verursacht. Die Folgen einer Aufhebung dieser - nicht realitätsgerechten - Annahme werden noch erörtert.

Vergröbernd, aber akzeptabel ist schließlich die Unterstellung, daß das verfügbare Alterseinkommen als die typischerweise bei Eintritt des Pflegefalls relevante Größe in einer festen und einheitlichen Relation (r) zu dem Erwerbseinkommen steht, das ein Empfänger im Durchschnitt erzielt hat. Im Rahmen dieser Annahmen läßt sich der von der Pflegeversicherung ausgehende Vorteil gegenüber einer versicherungslosen Ausgangslage mit Mindestsicherung zunächst anhand zweier Grenzfälle beschreiben ${ }^{29}$ :

Im Fall $A$ ist das Aktiveinkommen ${ }^{30}$ mit $M / r$ gerade so hoch, daß die Ruhestandsbezüge dem allgemeinen Existenzminimum $\mathrm{M}$ entsprechen. ${ }^{31}$ Tritt der Pflegefall ein, so sind - ohne Versicherung - die gesamten Pflegekosten von der Sozialhilfe zu bestreiten. Existiert eine Versicherung, so wird der Sozialhilfetransfer durch eine Versicherungsleistung in gleicher Höhe ersetzt; der Zugewinn für den Versicherten ist gleich Null.

\footnotetext{
${ }^{29}$ Die Darstellung entspricht in etwa der bei S.Winters (1993), S. $301 \mathrm{f}$.

${ }^{30}$ Der Übersichtlichkeit halber wird hier die Beitragszahlung durch (nicht pflegebedürftige) Rentner nicht behandelt, obwohl auch diese beitragspflichtig sein werden und sollten (siehe Abschnitt 4.2.3). Der Sache nach wäre eine entsprechende Ergänzung leicht möglich; ihr Fehlen berührt das hier angestellte Kalkül im übrigen nicht.

${ }^{31}$ Das Existenzminimum gilt sowohl bei Pflegebedürftigkeit als auch ohne sie und ist daher nicht Teil der Versicherungsleistung. Auch iim Fall der Heimpflege hat der Betroffene sinnvollerweise diejenigen Kosten zu tragen, die ihm auch ohne Pflegebedürftigkeit entstünden.
} 
Im Fall B beträgt das Erwerbseinkommen $(\mathrm{P}+\mathrm{M}) / \mathrm{r}$, das Alterseinkommen mithin $\mathrm{P}+\mathrm{M}$. Sein Empfänger kann im Pflegefall ohne Versicherung die Kosten gerade selbst tragen; ein Mindestsicherungs-Anspruch entsteht nicht. Es tritt also durch die Pflegebedürftigkeit eine Einkommenseinbuße in Höhe von P ein, die bei Existenz einer Versicherung vermieden wird. Einen Vorteil in gleicher Höhe haben Bezieher noch höherer Einkommen.

Zwischen den durch A und B markierten Grenzen steigt der durch die Pflegeversicherung gegenüber der bloßen Mindestsicherung erzielte Vorteil linear an: Jede zusätzliche Geldeinheit an (Alters-)Einkommen ist in diesem Bereich gleichermaßen vom Verzehr durch Pflegekosten bedroht, solange keine Versicherung existiert. Sollen nun die Beiträge zur Pflegeversicherung im Sinne des Äquivalenzprinzips der individuellen Leistungserwartung entsprechen, so müssen sie einen konstanten Anteil des Einkommens zwischen den genannten Grenzen ausmachen. Die Grenzeinkommen $\mathrm{M} / \mathrm{r}$ und $\mathrm{M}+\mathrm{P} / \mathrm{r}$ stellen folglich die untere und die obere Beitragsbemessungsgrenze dar: Bis $\mathrm{M} / \mathrm{r}$ ist die von der Versicherung zusätzlich erwartete Leistung Null, oberhalb von $\mathrm{M}+\mathrm{P} / \mathrm{r}$ steigt sie nicht mehr an, da der über $\mathrm{M}+\mathrm{P}$ hinausgehende Teil des entsprechenden Alterseinkommens vom Pflegefall unberührt bleibt.

Einkommensschwankungen brauchen dabei übrigens nicht beachtet zu werden. Das Alterseinkommen richtet sich annahmegemäß nach dem durchschnittlichen Erwerbseinkommen, für die Beiträge gilt dies in der Summe aufgrund des linearen Tarifs ebenso. Zu gewissen Verzerrungen kommt es nur dann, wenn das Einkommen zeitweilig die Beitragsbemessungsgrenzen durchbricht.

Zu dieser Basisversion des Ergebnisses sind einige Kommentare in bezug auf die ihm zugrundeliegenden Prämissen nötig: Zunächst geht die Äquivalenzrechnung freilich nur dann auf, wenn alle Versicherten schon mit Beginn ihrer Erwerbstätigkeit der Versicherung beitreten. Andernfalls findet eine Umverteilung zugunsten derjenigen statt, die erst in höherem Alter mit den Beitragszahlungen beginnen.

Dies ist dann der Fall, wenn die Versicherung zu einem beliebigen Zeitpunkt erst eingeführt wird. Wer in diesem Moment schon im Erwerbsleben steht, müßte also für das bereits erzielte und bislang beitragsfreie Einkommen Beiträge "nachzahlen". Das Pareto-Kriterium wird durch einen Verzicht auf die Berücksichtigung des Alters 
bei der Beitragsbemessung aber dann nicht verletzt, wenn das Umlageverfahren ${ }^{32}$ gilt: Die mit ihm verbundene Begünstigung der bei Einführung Älteren führt erst dann zu einer entsprechenden Benachteiligung anderer, wenn die umlagefinanzierte Versicherung abgeschafft wird, im Idealfall mithin nie. Falls doch, hat die Generation den Schaden, die kurz vor Schluß noch Beiträge zu zahlen hatte, denen keine Leistungen mehr gegenüberstehen.

Gravierendere Folgen für die die Ausgestaltung der Beiträge in bezug auf Einkommen hat es, wenn die Annahme der in allen Fällen einheitlichen Pflegekosten aufgegeben wird. $\mathrm{Da} ß$ es sich dabei um eine recht gängige Vereinfachung handel ${ }^{33}$, ändert nichts an der Tatsache, daß das Pflegerisiko in Wirklichkeit weniger schlicht strukturiert ist. Je nach Ausmaß der Bedürftigkeit werden auch die Kosten der Pflege und damit die Versicherungsleistungen unterschiedlich hoch sein. Es wäre also sachgerecht, jeder Pflegestufeeine eigene Eintrittswahrscheinlichkeit zuzuordnen. Die Konsequenz dieser Modifikation sei an einem einfachen Beispiel illustriert:

Es zerfalle der insgesamt mit einer Wahrscheinlichkeit p eintretende Pflegefall in die Varianten "leichte" und "schwere Pflegebedürftigkeit", deren Wahrscheinlichkeit jeweils $\mathrm{p} / 2$ betrage. Die vollen Pflegekosten P entstehen nur im schweren Fall, im leichteren beträgt der Schaden $\mathrm{P} / 2$. Es ändert sich dadurch nichts an den oben festgelegten Beitragsbemessungsgrenzen und auch nichts am Versicherungseffekt im unteren Bereich des beitragspflichtigen Einkommens. Dies gilt aber nur bis zu einer neu einzuführenden Grenze bei $(M+P / 2) / r$, der das Alterseinkommen $M+P / 2$ entspricht. Für den diese Schwelle übersteigenden Teil des Alterseinkommens ist es nur noch halb so wahrscheinlich wie im Standardfall, daß es aufgrund von Pflegebedürftigkeit verlorengeht, wenn keine Versicherung besteht. Analog ändert sich der Erwartungswert der Versicherungsleistungen und es müßte zur Wahrung des Äquivalenzprinzips auf diese Einkommensteile ein um die Hälfte niedrigerer Beitragssatz angewandt werden. Die durchgehend lineare Beziehung geht verloren.

Ein Versuch, diese Überlegung bei der Beitragsgestaltung konsequent zu beherzigen, würde darauf hinauslaufen müssen, daß es ebensoviele Beitragssätze wie Pflegestufen

\footnotetext{
${ }^{32}$ Zur Klarstellung: Abgesehen von diesem gedanklichen Schlenker ist die Wahl des Finanzierungsverfahrens für die in diesem Abschnitt verfolgte Argumentation unerheblich. Umverteilungsvorgänge zwischen Generationen sind hier ausgeblendet; sie kommen im Abschnitt 4.2.3 zur Sprache.

${ }^{33}$ siehe beispielsweise W. Buchholz/W. Wiegard (1992), S. 442
} 
bzw. Versicherungsleistungshöhen gäbe. Jedem dieser Beitragssätze würde ein bestimmter Teil des Einkommens unterworfen, wobei die Grenzbelastung mit steigendem Einkommen fiele ${ }^{34}$. Abgesehen von den praktischen Schwierigkeiten bei der korrekten Bestimmung eines solchen Tarifs wäre bei monatlicher Festsetzung und Einziehung der Beiträge die Folge, daß die gesamte Beitragslast nicht mehr allein von der Summe der Einkünfte, sondern auch von deren zeitlicher Verteilung abhinge. Strenggenommen müßte man diesen Effekt durch gesonderte $\mathrm{Zu}$ - oder Abschläge bei den Prämien jeweils nachträglich eliminieren. Nur so könnte sichergestellt werden, daß nach Abschluß der aktiven Zeit die insgesamt geleisteten Beiträge mit der Leistungserwartung übereinstimmen.

Bis hierher kann festgehalten werden: Da eine realitätsgerechte Darstellung der Referenzlage ohne spezifische Pflegeversicherung die Wirkung der staatlichen Mindestsicherung einschließen muß, hängt der daran gemessene Schutzeffekt der Versicherung vom Einkommen ab: Je höher dieses, desto geringer ist die potentielle Bedeutung der Mindestsicherung und desto stärker kommt der Versicherungsschutz effektiv zum Tragen. Im Sinne des Pareto-Kriteriums, also ohne Rücksicht auf spezifische Verteilungsziele, sind die Beiträge an der effektiven Schutzwirkung auszurichten und daher ihrerseits an das Einkommen zu koppeln.

Allerdings differiert der Schutzeffekt nicht über die gesamte Einkommensskala, sondern nur zwischen zwei Grenzwerten, die sich anhand des Existenzminimums bzw. des Maximums der Pflegekosten pro Zeiteinheit bestimmen lassen. Damit sind zwei Bemessungsgrenzen für die Einkommensabhängigkeit des Versicherungsbeitrags gegeben. Der geeignete Verlauf des Beitragstarifs zwischen ihnen hängt von der inneren Struktur des Pflegerisikos in Hinblick auf die Kosten und Wahrscheinlichkeiten der einzelnen Bedürftigkeitsstufen ab. Nur wenn dem Pflegerisiko vereinfachend ein standardisierter Kostensatz zugerechnet wird, ergibt sich ein zum beitragspflichtigen Einkommen proportionaler Verlauf als die beste Lösung.

\footnotetext{
${ }^{34}$ Dies kurz gesagt deswegen, weil die Wahrscheinlichkeit einer höheren Pflegestufe zwangsläufig geringer ist als die Wahrscheinlichkeit, überhaupt pflegebedürftig zu werden. Untere Einkommensteile sind von jedem Pflegefall bedroht, obere nur vom schweren.
} 


\subsubsection{Ergänzende Überlegungen zur Frage eines Staatszuschusses}

Die bisherige einfache Darstellung ist in zwei Punkten ergänzungsbedürftig: Ausgeblendet war zum einen die Finanzierungsseite der Mindestsicherung, die für ein vollständiges Pareto-Kalkül ebenfalls in Rechnung gestellt werden muß. Zum anderen ist das Problem zu lösen, daß die auf dem hier beschrittenen Weg be

stimmten Beiträge nicht zur Deckung der gesamten Pflegekosten ausreichen. Zwischen beiden Punkten besteht ein enger Zusammenhang.

Eine Diskrepanz zwischen Beitrag und Leistungsvolumen entsteht zunächst in Höhe der Verwaltungskosten (und ggfs. des Gewinns) einer Versicherung. Diesem Posten ist in einer Gesamtbetrachtung aber der Verwaltungsaufwand gegenüberzustellen, der mit Einführung der Pflegeversicherung bei den Trägern der Mindestsicherung entfällt. Wenn der Saldo beider Größen Null beträgt ${ }^{35}$, handelt es sich bei diesem Punkt um ein reines Verteilungsproblem.

Eine absolute Übereinstimmung zwischen Beitrag und Leistungserwartung scheitert aber vor allem an der oben erläuterten Differenz zwischen dem jeweiligen Gesamtschaden und der effektiven Versicherungsleistung. Während die Versicherung die gesamten Pflegekosten übernehmen soll, beziehen sich die nach dem vorgeschlagenen Verfahren ermittelten Beiträge nur auf den Teil davon, der nicht andernfalls von der Mindestsicherung getragen würde. Wenn also das gesamte Leistungsvolumen aus Beitragsmitteln bestritten werden soll, müssen die Beiträge höher liegen als die jeweiligen Erwartungswerte für die effektive Versicherungsleistung. Welcher Verteilungsschlüssel sollte bei der Schließung dieser Lücke zu Anwendung kommen?

Entscheidend ist in dieser Frage die Finanzierungsinzidenz der Mindestsicherung. Entlastend wirkt die Pflegeversicherung im hier aufgespannten Rahmen nämlich auch auf diejenigen, die ohne eine solche Einrichtung über Steuerzahlungen zum staatlich finanzierten Teil der Pflegelasten (einschließlich der erwähnten Verwaltungskosten) beizutragen hätten. Ließe sich diese Entlastung für jeden Versicherten korrekt quan-

\footnotetext{
${ }^{35}$ Dies ist freilich eine recht willkürliche Annahme. Als grobe Näherung erscheint sie aber insofern haltbar, als bei der Mindestsicherung zwar eine geringere Zahl von Fällen zu verwalten ist, der Aufwand pro Fall aufgrund der erforderlichen umfassenden Bedürftigkeitsprüfung aber deutlich größer sein dürfte.
} 
tifizieren und seinem wie oben ermittelten "Normal-Beitrag" zuschlagen, so wäre die Finanzierungslücke geschlossen und die Wohlfahrtsverbesserung gewährleistet.

Dies aber wird nicht gelingen. Abgesehen von der praktischen Unvorstellbarkeit einer solchen höchst aufwendigen und intransparenten doppelten Beitragsrechnung ist zu bedenken, daß sich die Lasten der Mindestsicherung unter Beachtung des Nonaffektations-Prinzips nicht einer einzelnen Steuer zurechnen lassen. Vielmehr müßte theoretisch der Gesamtbeitrag jedes einzelnen zu den Staatseinnahmen ermittelt werden, was eine genaue Kenntnis von Konsumgewohnheiten und Lebensumständen voraussetzt. Jede dort oder in den Einnahmestrukturen des Staates eintretende Veränderung müßte nachvollzogen und zudem berücksichtigt werden, daß der Staat einen erheblichen Teil seiner Ausgaben auf Basis von Krediten tätigt.

Angesichts der offenkundigen Absurdität eines solchen Vorgehens bietet sich ein unproblematischer und mit der angestrebten Wohlfahrtsverbesserung ebenfalls kompatibler Ausweg an: ein Zuschuß des Staates an die Versicherung im Umfang der eingesparten Mindestsicherungs-Transfers und der Verwaltungskosten, die auf ihre Gewährung entfielen. Bei unveränderter Steuerlast im Vergleich zur Referenzlage könnten die Beiträge so auf dem Niveau der tatsächlichen Leistungserwartung gehalten werden.

Leider aber kollidiert diese aus der hier eingenommenen Perspektive so sinnfällige Lösung mit den Überlegungen, die weiter oben zur Ablehnung einer vollständig steuerfinanzierten Pflegesicherung geführt haben. Auch eine nur partielle und nach zumindest theoretisch - klaren Regeln bestimmte finanzielle Beteiligung des Staates am Leistungsgeschehen schmälert im Kern die Verläßlichkeit des Schutzversprechens. ${ }^{36}$

Es muß daher festgestellt werden, daß sich eine detaillierte und praktikable Handlungsanweisung für die Finanzierungsseite aus der Verfolgung des Pareto-

\footnotetext{
${ }^{36}$ Siehe etwa F. Pirkl (1986), S. 10: "Wer also bei seiner Konzeption auf staatliche Zuschüsse setzt, muß sich darüber im klaren sein, daß er damit erhebliche, vom jeweiligen Verlauf des Steueraufkommens abhängige Risiken einbaut." Hier spricht wohlgemerkt ein selbst in der Exekutive tätiger Politiker. Die allenthalben konstatierte "Krise der Staatsfinanzen" hat sich seit dem Zeitpunkt dieser Äußerung noch erheblich verschärft.
} 
Kriteriums als allokativer Zielvorgabe letztlich nicht ableiten läßt. Sofern allerdings dem Steuersystem als Ganzem eine nichtregressive Belastungswirkung zugeschrieben werden kann, was schon aufgrund der zumeist zentralen Rolle der progressiven Einkommensteuer plausibel erscheint, so unterstreicht die Einbeziehung des zuletzt diskutierten Aspekts in qualitativer Hinsicht die Forderung nach einer positiven Abhängigkeit der Beiträge vom Einkommen. Unklar bleibt hingegen die genaue Form des dabei zu wählenden Beitragstarifs. Zum Trost sei der enttäuschte Leser darauf hingewiesen, daß ein Beitrag, der im Einzelfall die Leistungserwartung zuzüglich ersparter Steuerzahlung übersteigt, keineswegs zwingend mit einer Schlechterstellung des derart Belasteten im Vergleich zur Ausgangslage ohne Versicherung verbunden ist. Eine Verletzung des Pareto-Kriteriums in solchen Fällen tritt nur dann ein, wenn die jeweilige Risikoaversion nicht stark genug ausgeprägt ist, um die Abweichung vom Äquivalenzbeitrag aufzuwiegen. ${ }^{37}$

\subsubsection{Die Wahl des Finanzierungsmodus im Lichte beider Kriterien}

Die Frage des Finanzierungsmodus hat in der Debatte um die Pflegesicherung in der Bundesrepublik breiten Raum eingenommen. Sie hat eine gewisse ideologische Aufladung auch dadurch erfahren, daß sie vielfach nicht unabhängig von anderen Gestaltungsparametern etwa in bezug auf die Trägerschaft (öffentlich vs. privat) oder die Beitragsbemessung behandelt worden ist. In der Regel wird dabei das Umlageverfahren in Entsprechung zur deutschen Sozialversicherungs-Tradition mit der (para)staatlichen Trägerschaft und einkommensabhängigen Beiträgen assoziiert, ein kapitalfundiertes Verfahren umgekehrt als typisches Element einer Marktlösung mit "risikogerechten" Prämien bewertet. ${ }^{38}$ Auch wenn diese Zuordnung mit den Merkmalskombinationen der wesentlichsten Lösungsvorschläge übereinstimmt, ist es für eine theoretische Analyse geboten, die Frage des Finanzierungsmodus separat zu behandeln. Von den oben aufgeführten Typen abweichende Kombinationen sind prinzipiell durchaus denkbar: So können auch die im vorigen Abschnitt als wünschenswert abgeleiteten einkommensabhängigen Beiträge durchaus der Kapitalansammlung dienen.

\footnotetext{
${ }^{37}$ vgl. H.-W. Sinn (1977), S. 529

${ }^{38}$ Dies zeigt und kritisiert z.B. W. Schmähl (1992), S. 29, v.a Fußnote 14.
} 
Idealtypisch kennzeichnen lassen sich die einander gegenüberstehenden Konzepte wie folgt: Sieht das Umlageverfahren einen "Ausgleich von Einnahmen und Ausgaben [der Versicherung, S.W.] innerhalb eines eng begrenzten, in der Regel ein Jahr umfassenden Zeitraums ${ }^{139}$ vor, so erfolgt bei kapitalfundierten Verfahren ${ }^{40}$ zunächst ein Ansparprozeß, nach dessen Abschluß die Leistungsgewährung aus den angesammelten Beitragsmitteln (und den darauf entfallenden Zinserträgen) beginnt.

Die in Deutschland geführte Kontroverse zwischen Anhängern beider Optionen war stark von der Frage geprägt, wie in denjenigen Pflegefällen Abhilfe geschaffen werden kann, die in der Anlaufphase eines Kapitaldeckungsverfahrens eintreten oder gegenwärtig bereits eingetreten sind. In Hinblick darauf muß ein Verfahren ineffektiv erscheinen, das seine volle Wirkung erst nach Jahrzehnten zu entfalten verspricht. Dieser Aspekt steht hier aber ebensowenig zur Debatte wie die heillos umstrittenen möglichen Nebenwirkungen eines Kapitalansammlungsverfahrens auf das wirtschaftliche Wachstum. ${ }^{41}$ Statt dessen soll im Anschluß an die Überlegungen der vorigen Abschnitte geprüft werden, inwieweit die dort aufgestellten Kriterien Argumente für oder gegen beide Finanzierungswege zu liefern vermögen. Zentral ist für diese Diskussion die - der aktuellen Wirklichkeit aller Industrieländer entsprechende Annahme schwankender Generationsgrößen, oder genauer: eines deutlich und anhaltend steigenden Bevölkerungsanteils alter Menschen.

Die Erfüllung des Pareto-Kriteriums mit Hilfe einkommensabhängiger Beiträge ist nämlich unter den Bedingungen des Umlageverfahrens sowie bei gegebenen Pflegekosten und altersspezifischen Pflegerisiken nur dann ohne weiteres gewährleistet, wenn sich die Bevölkerung stationär entwickelt, also jeder Altersjahrgang gleich stark besetzt ist. Andernfalls kommt es zu Abweichungen des tatsächlichen Beitrags vom

\footnotetext{
${ }^{39}$ ebenda, S. 25

${ }^{40}$ Zur Unterscheidung verschiedener Varianten kapitalgedeckter Verfahren, bei denen der Kapitalstock unterschiedliche Ausmaße annimmt, siehe etwa G. Heubeck (1982), S. $294 \mathrm{ff}$.

${ }^{41}$ So erklärt zum Beispiel der Wissenschaftliche Beirat beim BMF (1990), S. 13 die mit dem Kapitaldeckungsverfahren seiner Ansicht nach verbundene "Erhöhung der gesamtwirtschaftlichen Sparleistung" für "sehr erwünscht". W. Schmähl (1992), S. 51 hingegen äußert unter Hinweis auf die in der Ansparphase eintretende Doppelbelastung, die ihrerseits ersparnismindernd wirken könne, "erhebliche Zweifel daran, ob es tatsächlich zu einer (gesamtwirtschaftlich relevanten) erhöhten Vermögensbildung kommt". Ähnlich argumentieren auch H. Ribhegge (1990) und G. Wagner (1991), S. 45, der überdies auf die Ungewißheit abstellt, "ob eine (evtl.,S.W.) erhöhte Sparsumme auch tatsächlich in Wachstum umgesetzt wird."
} 
äquivalenten, wobei solche Generationen benachteiligt werden, die im Vergleich zu den vorangegangenen zahlenmäßig schwach sind. Dieser Sachverhalt ist aus der Diskussion um die Umlagefinanzierung der Rentenversicherung bekannt und von BREYER $^{42}$ in ausführlicher Form auf den Bereich der Pflegesicherung übertragen worden.

Allerdings ist auf einen wesentlichen Unterschied zwischen beiden Bereichen hinzuweisen, den BREYER ignoriert: Anders als im Fall der Altersversorgung stehen sich bei der Pflegeversicherung nicht zwei Generationen gegenüber, von denen eine die gesamte Beitragslast trägt, während die andere ausschließlich (und geschlossen) Leistungen in Anspruch nimmt. Abgesehen von der Tatsache, daß Pflegebedürftigkeit gelegentlich auch in jungen Jahren auftritt, liegt der Unterschied darin, daß die (nicht pflegebedürftigen) Alten weiterhin Beiträge entrichten. ${ }^{43}$ Inwieweit dies die intergenerative Beitragsverzerrung mildert, läßt sich an einem sehr schematischen Zahlenbeispiel zeigen:

Als Folie sei eine Rentenversicherung betrachtet, bei der eine Generation A die Alterseinkünfte einer Generation $\mathrm{R}$ finanziert, während ihre eigenen Renten von der Generation B aufzubringen sind. B und A sind gleich groß. Als konstant unterstellt seien dabei das Lohn- und das Rentenniveau sowie die Erwerbsquote. Sind A und $\mathrm{R}$ gleich stark, so werden die Beitragszahler A insgesamt unter diesen Umständen ebensoviel zurückerhalten, wie sie aufgewandt haben. Umfaßt ihre Generation hingegen nur halb soviele Mitglieder wie die der Rentner, so müssen sie genau doppelt so viel einzahlen, wie sie an Leistungen selbst erhalten werden. Entsprechend verdoppelt sich in diesem Fall auch der Beitragssatz.

In der Pflegeversicherung stellt sich ein analoger Fall wie folgt dar: In der Referenzlage stehen 100 Angehörigen der "jungen" Generation ebensoviele "Alte" gegenüber. Das Pro-Kopf-Einkommen beträgt für die Jungen 2, für die Alten eine Geldeinheit. Die Pflegewahrscheinlichkeit sei für die ersteren Null, für die letzteren zehn Prozent. Die Pflegekosten pro Fall belaufen sich auf $3 \mathrm{GE}$, in der Summe also auf $30 \mathrm{GE}$. Werden sie zur Gänze über vollständig einkommensproportionale Beiträge aufgebracht, so

\footnotetext{
${ }^{42}$ F. Breyer (1991)

${ }^{43}$ Breyer trifft hingegen sehr beiläufig die Annahme, daß Beiträge "nur von Aktiven erhoben werden" (ebenda, S. 89). Inzwischen hat er in Reaktion auf eine entsprechende Kritik von R. Eisen (1994), S. 68 zugestanden, daß dies nicht korrekt ist (siehe F. Breyer (1994), S. 73).
} 
erfordert dies einen Beitragssatz von $10 \%$, der wiederum das Äquivalenzniveau markiert.

Wird nun ebenso wie oben in bezug auf die Rentenversicherung angenommen, die junge Generation sehe sich einer doppelt so großen Gruppe von Alten gegenüber, so verdoppelt sich freilich auch die gesamte Pflegelast. Da aber die zusätzlichen Alten mit ihrem Standardeinkommen von jeweils einer Einheit auch beitragspflichtig sind ${ }^{44}$, kommt es nicht wie im Fall der Rente zu einer Verdoppelung des Beitragssatzes. Dieser ergibt sich nun vielmehr als $60 \mathrm{GE} / 400 \mathrm{GE}=15 \% .{ }^{45}$

Damit ist gezeigt, daß die Lastenverschiebung zwischen den Generationen, wie sie das Umlageverfahren bei generationsspezifischen Risiken im Falle schwankender Jahrgangsstärken mit sich bringt, bei der Pflegeversicherung schwächer ausgeprägt ist als bei der Rentenversicherung, wo die Zugehörigkeit zu einer bestimmten Altersgruppe praktisch identisch ist mit dem Eintritt des "Risikos". Vollends aufgehoben ist der Effekt gleichwohl nicht. Somit besteht durchaus die Gefahr, daß (weniger risikoscheue) Angehörige einer relativ schwach besetzten Generation ihre Beitragsbelastung in dem Sinn als "überhöht" empfinden, daß sie einen versicherungslosen Zustand vorziehen würden. In solchen Fällen wäre das Pareto-Kriterium verletzt.

Um das Ausmaß dieser Effizienzstörung einschätzen zu können, bedarf es allerdings einer Berücksichtigung derjenigen externen Effekte, die mit der Versicherung für Angehörige anderer als der jeweils versicherten Generation selbst verbunden sein können. Es ist naheliegend, die Existenz solcher Effekte zu vermuten, da bei politischen Forderungen nach Errichtung eines Systems zur Sicherung der Pflege stets auch eine Verbesserung der Lage pflegender Angehöriger als Ziel genannt wird. ${ }^{46}$ Bei ihnen aber handelt es sich zu einem großen Teil um Kinder und Schwiegerkinder der

\footnotetext{
${ }^{44}$ Im System der deutschen Sozialversicherung ist es freilich so, daß mit der Hälfte dieser Beiträge die Rentenversicherung belastet wird (vgl. B. Felderer (1992), S. 17).

${ }^{45}$ In diesem einfachen Beispiel ist der zahlenmäßigen Durchsichtigkeit halber impliziert, daß auch die Pflegebedürftigen selbst weiterhin Beiträge entrichten. Tun sie es nicht, so ergibt sich bei Verdoppelung der "alten" Generation ein Beitragssatz von rd. 15,8 statt 10,3\%, was einer Steigerung von rd. 52,5 statt $50 \%$ gleichkommt. Die Kernaussage bleibt mithin unberührt.
}

${ }^{46}$ siehe etwa Bundesministerium für Arbeit und Sozialordnung (1992), S. 8f. 
Betroffenen ${ }^{47}$, mithin typischerweise um Angehörige der Generation, die im erwerbsfähigen Alter steht und die Hauptlast der Umlage trägt.

Zwar wurde weiter oben Wert auf die Feststellung gelegt, daß die Übernahme der Pflege durch die eigenen Kinder keineswegs unbesehen als Normalfall betrachtet werden darf, so daß die Existenz von Kindern als Quasi-Versicherung gelten könnte. Gleichwohl wird die häusliche Pflege aus Sicht der Kinder in den meisten Fällen als eine denkbare Variante antizipiert, über deren Realisierung nach Eintritt des Pflegefalls im Lichte der dann herrschenden Umstände (Grad der Bedürftigkeit, Verhältnis zum Pflegebedürftigen, eigene soziale, ökonomische und gesundheitliche Lage, Qualität der Alternativen zur Hauspflege etc.) entschieden wird.

Der mögliche Eintritt einer Situation, in der sie sich zur Übernahme der Pflegeverantwortung moralisch genötigt sähen, stellt also für die Kinder selbst eine Art "Risiko" dar, für das ein Sicherungsbedürfnis angenommen werden kann. Dieses ist insoweit gedeckt, wie der den Eltern zuteil werdende Versicherungsschutz unterstützende ambulante Leistungen für den Fall der grundsätzlich innerfamiliär organisierten Pflege einschließt.

Zugunsten von Kindern, die zur Übernahme der Pflege nicht bereit oder imstande sind, tritt ein weiterer externer Effekt dann auf, wenn der (potentiell) Pflegebedürftige ihnen ein Erbe zu hinterlassen verspricht. Ohne Versicherung wäre es vom - oftmals restlosen - Verzehr durch die Pflegekosten bedroht. ${ }^{48}$ Ein dritter Effekt tritt dann hinzu, wenn Angehörige vor Eintritt der Mindestsicherung unterhaltsrechtlich gezwungen werden können, sich an Pflegekosten zu beteiligen. Auch dann profitieren Kinder davon, daß ihre Eltern pflegeversichert sind.

\footnotetext{
${ }^{47}$ siehe U. Schneekloth/P. Potthoff(1993), S. 129: Bei den über 80jährigen Pflegebedürftigen außerhalb von Heimen fungieren in Deutschland in zwei von drei Fällen die Tochter, die Schwiegertochter oder gelegentlich auch - der Sohn als Hauptpflegekraft.

${ }^{48}$ Zugespitzt formuliert M.V. Pauly (1990), S. 166: "The main benefit of more extensive LTC (long term care, S.W.) insurance (...) would be the benefit obtained by risk-averse heirs from reducing the risk attached to the inheritance they will receive." - Zwar ist anzunehmen, daß die Beiträge zur Pflegeversicherung den Aufbau der Erbmasse hemmen. Aber aufgrund ihrer Risikoscheu sind die modellhaften Erben diesen Preis zu zahlen bereit. Daß es sich bei diesem Aspekt um den "main benefit" handele, behauptet Pauly aufgrund von zweifelhaften Annahmen, die in Abschnitt 4.3.3.4 zur Sprache kommen.
} 
Eine korrekte "Internalisierung" solcher Effekte im Zuge einer Beteiligung der Kinder an den elterlichen Versicherungsbeiträgen wäre praktisch außerordentlich schwierig. Bei gleichbleibenden Generationsstärken ist sie aber auch nicht erforderlich, da der von einer Generation zu entrichtende Beitragsanteil zugunsten der Eltern der Summe nach dem entspräche, was sie von ihren Kindern wiederum einfordern könnten.

Anders verhält es sich im Fall von Generationen schwankender Größe: Je schmaler eine Kindergeneration im Vergleich zu der ihrer Eltern, desto größer ist für sie das Ausmaß der externen Effekte. Je geringer die Kinderzahl pro Elternteil, desto größer die Wahrscheinlichkeit, eine evtuelle Hauspflege selbst bzw. allein ausüben zu müssen, desto größer auch unter sonst gleichbleibenden Bedingungen das individuelle Erbteil bzw. die Unterhaltspflicht.

Es zeigt sich also, daß der intergenerative Belastungsverlauf im Umlageverfahren dem Schwankungsmuster im Ausmaß der externen Effekte entspricht. Ein Versuch, diese Effekte zu quantifizieren, würde allerdings im Gestrüpp der nötigen Fallunterscheidungen steckenbleiben. Ohne weiteres lassen sich Gruppen konstruieren, für die die aufgeführten Zusammenhänge irrelevant $\operatorname{sind}^{49}$ und die deswegen im Endeffekt doch aus dem Muster der Pareto-Verbesserung herausfallen können. Gleichwohl mindert die implizite Berücksichtigung externer Effekte im Umlageverfahren das mögliche Ausmaß einer solchen Verletzung des Kriteriums bei wechselnden Größenverhältnissen zwischen den Generationen.

Abschließend bleibt - als Nachtrag zur Diskussion um die Beitragsgestaltung - die Frage zu klären, inwiefern die externen Effekte die dort dargelegte Vorteilhaftigkeit einkommensabhängiger Beiträge zu stören geeignet sind. Am einfachsten ist sie in bezug auf die innerfamiliären Unterhaltsleistungen zu klären. Diese sind selbst einkommensabhängig, der "Wert" ihrer Vermeidung fügt sich daher in das Beitragsmuster ein. Tendenziell wird dies auch für die häuslichen Pflegehilfen gelten, da die Zahlungsbereitschaft für solche Dienste und damit der Nutzen ihrer Bereitstellung durch die Versicherung im allgemeinen mit dem Einkommen ansteigt. Dies gilt vor allem dann, wenn die Möglichkeit der Einkommenserzielung gerade davon abhängt, ob eine professionelle Unterstützung bei der häuslichen Pflege erfolgt. Als mögliche Störgröße bleibt somit nur der Aspekt des Erbes übrig. Für sich genommen rechtfertigt

\footnotetext{
${ }^{49}$ Man denke etwa an Vollwaisen.
} 
er die Einkommensabhängigkeit der Beiträge nur dann, wenn sich eine positive Korrelation zwischen dem Vermögen der Eltern und dem Einkommen der Kinder feststellen ließe.

Ob diese Überlegung ein wirklich schlagendes Argument für das Umlageverfahren und damit gegen das Kapitaldeckungsverfahren abgibt, mag angesichts der fehlenden Meßbarkeit des externen Effekts dahingestellt bleiben. Immerhin aber entzieht sie der Gegenthese den Boden, derzufolge das Kapitaldeckungsverfahren gerade unter dem Aspekt der Beitragsäquivalenz über die Generationen hinweg vorzuziehen sei. Außerdem kann von hier aus erneut das Kriterium der Verläßlichkeit in den Blick genommen werden.

Die demographische "Anfälligkeit" des Umlageverfahrens wird nämlich nicht nur als Störung der Beitragsäquivalenz oder als Quelle unerwünschter Verteilungswirkungen ${ }^{50}$ diskutiert. Vielmehr müsse in deren Folge damit gerechnet werden, daß "die langfristige Tragfähigkeit des Generationenvertrags (...) gefährdet" sei. ${ }^{51}$ Eine per Umlage organisierte Absicherung gegen das Pflegerisiko sei daher "schon bald zum Scheitern verurteilt"..$^{52}$

Die oben angestellten Überlegungen zu den externen Effekten der Pflegeversicherung sind geeignet, derlei Befürchtungen zu entkräften, und zwar unabhängig von den generellen Fragen, ob die heraufbeschworene Abschaffung einer Sozialversicherung überhaupt juristisch möglich und politisch durchsetzbar wäre und inwieweit ihr eine "Solidarität" zwischen den Generationen entgegensteht. Die "junge" Generation nämlich, von der ein solcher Schritt ausgehen müßte ${ }^{53}$, wäre von den Folgen in erheblichem Maße selbst betroffen. Da diese potentielle Rückwirkung wie gezeigt umso stärker ausfällt, je stärker das Übergewicht der "Alten" und je größer damit die Beitragsbelastung und der von ihr ausgehende "Kündigungsanreiz" sind, erscheint die

\footnotetext{
${ }^{\text {so }}$ so F. Breyer (1991), S. 100

${ }^{51}$ So wird die Auffassung des Wiss. Beirates beim BMF wiedergegeben in den BMF-Finanznachrichten Nr.33/93 vom 30. April 1993.

${ }^{52}$ Frankfurter Institut (1991), S. 6

${ }^{53}$ Nebenbei ist zu bedenken, daß Versicherte in mittleren Altersgruppen bei der Entscheidung für oder gegen eine Aufkündigung der Versicherung auch die von ihnen bereits gezahlten Beiträge ins Kalkül ziehen. Schon deswegen und in Hinblick auf die Stärke der alten Generation selbst ist kaum anzunehmen, daß eine Mehrheit gegen die Versicherung zustande käme.
} 
Sorge um die dauerhafte Existenzfähigkeit der umlagefinanzierten Versicherung unbegründet. Damit ist freilich nicht bestritten, daß es (auch) im Rahmen eines umlagefinanzierten Systems zu gewissen Leistungseinschränkungen kommen kann. Deren Art und Ausmaß wird am Beispiel der Niederlande zu evaluieren sein, wo der Beitragsdruck in der Tat über Jahrzehnte hinweg gestiegen ist.

Aus der - umstrittenen ${ }^{54}$ - "Immunität" des Kapitaldeckungsverfahrens gegenüber demographischen Spannungen kann also keine Überlegenheit in Hinblick auf die Bestandssicherheit der Pflegesicherung abgeleitet werden. Statt dessen ist ein anderes von der Bevölkerungsentwicklung unabhängiges - Problem zu nennen, das die Verläßlichkeit der Leistungsstandards unter den Bedingungen des Kapitaldeckungsverfahrens in Frage stellt:

Für ein kapitalfundiertes Verfahren nämlich muß zur korrekten Festsetzung der Prämien bereits Jahrzehnte vor dem Zeitpunkt der Leistungsgewährung das Mittelvolumen fixiert werden, das pro Kopf einer Alterskohorte zu Pflegezwecken zur Verfügung stehen soll. Damit es dem späteren Bedarf im Sinne der Zielvorgabe entspricht, sind also langfristige Prognosen sowohl über die zu erwartende Fallzahl als auch über die pro Fall benötigten Leistungsmengen und -formen sowie die spezifische Preisentwicklung erforderlich.

Auf die tatsächliche Entwicklung dieser Größen wirkt eine Fülle von Faktoren ein. So wird die Fallzahl etwa durch die Entwicklung von Mortalität und Morbidität, die Preisbewegung unter anderem maßgeblich durch die sektorspezifische Lohnentwicklung bestimmt. Umfang und Profil des Leistungsbedarfs pro Fall hängen von geriatrischen Erkenntnissen und wiederum von der zukünftigen Lebenserwartung bzw. davon ab, wie sich deren Entwicklung zu derjenigen des durchschnittlichen Gesundheitszustands pro Altersgruppe verhält. Schon die Frage, ob der Anteil der "gesunden Jahre" aboder zunimmt, wenn die Lebenserwartung ansteigt, ist nach wie vor ungeklärt..$^{55}$

\footnotetext{
${ }^{54}$ Zur Kritik siehe etwa H. Ribhegge (1990), S. 363ff., der unter anderem darauf hinweist, daß es bei Bevölkerungsrückgang zu einer allmählichen Entwertung des Deckungskapitals über sinkende reale Zinsen kommen kann.

ss Einen Überblick über die sehr unterschiedlichen Ergebnisse empirischer Studien dazu geben J.-M. Robine/E. Cambois (1994).
} 
Was die derart komplex bedingte Gesamtentwicklung des zur Bedarfsdeckung erforderlichen Leistungsumfangs betrifft, so ist mithin "zu befürchten, daß [es] angesichts der Vielzahl der Einflußgrößen (...) nicht möglich ist, die künftige Entwicklung ausreichend zu antizipieren". ${ }^{56}$ In dem Maße aber, wie die Festsetzung der Prämien im Kapitaldeckungsverfahren auf zu niedrigen Bedarfsprognosen beruht und nachträgliche Beitragserhöhungen nicht durchgesetzt werden können oder sollen, kommt es zwangsläufig ${ }^{57} \mathrm{zu}$ Leistungsminderungen in einer oder mehreren der beschriebenen Spielarten. ${ }^{58}$ Insofern ist das Kapitaldeckungsverfahren unter dem Gesichtspunkt der Verläßlichkeit als problematisch einzustufen.

\subsection{Effizienz in Hinblick auf die Gefahr des Moral hazard}

\subsubsection{Einleitung}

Die Existenz einer Versicherung erzeugt auf seiten der Versicherungsnehmer regelmäßig Verhaltensänderungen. Abstrakt formuliert stellt der Beitritt zur Versicherung eine "Verbesserung der Informationslage" 59 dar, auf deren Basis der Versicherte agiert. Im Grundsatz ist ein solcher Effekt keineswegs wohlfahrtsschädlich, sondern erwünscht: Wenn aufgrund der Schutzzusagen das Verhalten dahingehend modifiziert wird, daß es weniger stark auf die Vermeidung oder Begrenzung des versicherten Schadens ausgerichtet ist ${ }^{60}$, so erzeugt dies einen Wohlfahrtsgewinn. Dies gilt jedenfalls dann, wenn die in diesem Sinne gestiegene Risikobereitschaft individuell und präzise beobachtet und bei der Beitragsgestaltung berücksichtigt werden kann und wird. Da sich der risikoscheue Versicherungsnehmer so verhalten wird, daß sich sein Nutzen erhöht, während der Versicherer aufgrund seiner Reaktionsmöglichkeiten letztlich

\footnotetext{
${ }^{56}$ W. Schmähl (1992), S. 52

${ }^{57}$ Dies gilt für ein konsequent beibehaltenes Kapitaldeckungsverfahren. Praktisch vorstellbar ist freilich auch, daß ein anfänglich so verfaßtes System in ein gemischtes Verfahren mit zunehmender Bedeutung von Umlagen übergeht. G. Wagner (1991), S. 44, verweist auf eine solche Entwicklung bei der privaten Krankenversicherung, wo ebenso wie im Pflegebereich "das notwendige Sparvolumen nicht prognostizierbar" sei.

${ }^{58}$ So argumentiert auch W. Albers (1993), S. 3.

59 Wolfgang Müller (1988), S. 140

${ }^{60}$ Wie M. Nell (1992), S. 90ff., zeigt, ist es in bestimmten Fällen auch möglich, daß die Bemühungen, den Eintritt des Schadens zu verhindern, mit wachsendem Versicherungsschutz zunehmen! An der allokativen Wertung ändert sich aber auch dann nichts.
} 
"allen Entscheidungen des VN gegenüber indifferent ist" ${ }^{61}$, erfüllt ein solcher Idealfall die Pareto-Bedingung und ist daher "allokativ erwünscht". ${ }^{62}$ SINN spricht sich dafür aus, den nach allgemeinem Verständnis negativ besetzten Begriff des "Moral hazard" nicht für diesen Effekt zu verwenden. ${ }^{63}$

Entscheidend und problematisch ist dabei indes die Bedingung der vollständigen Informationssymmetrie zwischen den Vertragspartnern. In der Wirklichkeit wird die Erfassung und beitragsmäßige Berücksichtigung des gesamten jeweils risikorelevanten Verhaltens durch den Versicherer nur in Grenzen überhaupt möglich und zudem mit beträchtlichen Kosten verbunden sein. So sieht sich der Versicherer allein mit dem Schadensergebnis konfrontiert, ohne zwischen der zufalls- und der verhaltensbedingten Komponente seines Zustandekommens differenzieren zu können. Wird daher eine auf Gruppen bezogene oder gänzlich undifferenzierte Durchschnittstarifierung gewählt, werden die Bemühungen zur Schadensvermeidung oder -begrenzung zu stark bzw. sogar vollständig zurückgedrängt. Ein solches Übermaß an "Sorglosigkeit" stellt den typischen Fall des "Moral hazard" dar. Seine Folge ist ein Anstieg der Versicherungsprämien, der das Potential der Versicherung, die Wohlfahrt zu steigern, schmälert oder schlimmstenfalls zunichte macht.

Ist also der Informationsrückstand des Versicherers unüberwindlich oder wird aus anderen Gründen von einer Differenzierung der Prämien abgesehen, so kann eine Selbstbehaltsregelung - die freilich auf Kosten der Schutzwirkung geht - dafür sorgen, daß es dennoch attraktiv bleibt, sich um die Verhütung des Schadens zu bemühen. Eine völlige Heilung der mit dem Moral hazard verbundenen Wohlfahrtseinbußen wird auf diesem Weg allerdings nicht gelingen ${ }^{64}$ Das gleiche gilt für eine progressive Staffelung der Beiträge in Abhängigkeit vom gewählten Deckungsgrad. ${ }^{65}$

\footnotetext{
${ }^{61}$ H.-W. Sinn (1977), S. 522. "VN" heißt Versicherungsnehmer.

${ }^{62}$ vgl. M. Nell (1992), S. 95

${ }^{63}$ Siehe H.-W. Sinn (1977), S. 521. Ebenso plädiert Nell (1992, S. 105ff.), der gleichzeitig eine Reihe verbreiteter Definitionen des moral hazard aufführt, die keine Unterscheidung zwischen allokativ sinnvollen und unerwünschten Verhaltensänderungen vornehmen.
}

${ }^{64} \mathrm{vgl}$. Sinn (1977), S. 526f.

${ }^{65}$ vgl. Nell (1992), S. 223 
Die bisherige Darstellung des "Moral hazard" bezog sich zunächst auf Verhaltensweisen, die ihren Platz vor der Wirkung des Zufalls haben, der letztlich darüber entscheidet, ob ein Schaden eintritt oder nicht. Andere Spielarten des moral hazard beziehen sich auf das Verhalten nach Eintritt des Schadensfalls. Solcher Moral hazard "ex post" 66 wird dann und nur dann relevant, wenn die Schadenshöhe und damit die fällige Versicherungsleistung nicht eindeutig bestimmbar ist. Er ist mithin die Folge von Unklarheiten bei der Spezifikation und Feststellung des Schadensereignisses und damit auch der Auszahlungsfunktion, wie sie sich in bestimmten Versicherungssparten schwer vermeiden lassen. Bei der Risiko-Lebensversicherung etwa stellt sich ein solches Problem naturgemäß nicht: Der Schadensfall "Tod" ist eindeutig definiert und wird mit einer fixierten Auszahlung verknüpft. ${ }^{67}$ Auch die Feststellung des Schadensereignisses bereitet hier keine Schwierigkeiten.

Eher ist der Moral hazard ex post etwa für die Krankenversicherung oder auch für die Kfz-Haftpflichtversicherung ${ }^{68}$ denkbar, soweit unterstellt werden kann, daß in beiden Fällen je verschieden aufwendige Wege zur Behebung eines "Schadens" beschritten und vom Versicherungsnehmer gewählt werden können. Solange die Versicherung in allen Fällen die Kosten trägt, besteht dann kein Anreiz, bei dieser Entscheidung das Preisverhältnis zwischen verschiedenen Optionen zu beachten bzw. die Leistungsmenge auf das jeweils Notwendige zu beschränken.

In der Debatte um die Pflegeversicherung in der Bundesrepublik ist auf die vermutete Gefahr derartiger Phänomene großes Gewicht gelegt worden: "Die ökonomische Qualität einer Pflegeversicherung wird (..) stark davon beeinflußt, ob und in welchem $\mathrm{Maß}$ es gelingt, den (..) Moral hazard-Effekt einzudämmen". ${ }^{69}$ Regelmäßig wird dabei "als empirischer Beleg" für die große Relevanz dieses Effekts "die Entwicklung in den Niederlanden angeführt". ${ }^{70}$ Inwieweit die dortige Erfahrung als Beleg in diesem Sinne wirklich taugt, wird noch kritisch zu prüfen sein. Ein eindeutiger Befund im

\footnotetext{
${ }^{66}$ vgl. F. Breyer/P. Zweifel (1992), S. 172

${ }^{68}$ siehe H.-W. Sinn (1977), S. $532 \mathrm{ff}$.
${ }^{69}$ A. Prinz (1987), S. 113
${ }^{70}$ C. Offe/W. Schmähl (1992), S. 7 (Fußnote 4)

${ }^{67}$ Sobald diese Bedingung etwa in der Weise durchbrochen ist, daß bei Unfalltod eine höhere Versicherungssumme gezahlt wird, kann ein moral hazard ex post nicht mehr restlos ausgeschlossen werden. Er bestünde darin, den eingetretenen Tod als Unfallfolge erscheinen zu lassen. 
Sinne eines "Nachweises" oder einer "Widerlegung" des Moral hazard wird sich aus der bisherigen niederländischen Praxis allein freilich nicht gewinnen lassen. Daher und auch zur Vorbereitung empirischer Analysen ist es zunächst angezeigt, modellhaft bzw. mit Hilfe von Plausibiltätsüberlegungen der Frage nachzugehen, welche der grundsätzlich denkbaren Typen von Moral hazard im Kontext der Pflege überhaupt als relevant erscheinen. Weiterhin wird versucht, zu einer Einschätzung über das jeweilige Ausmaß solcher Relevanz zu gelangen.

Bei der Anwendung der oben angestellten allgemeinen Überlegungen auf den Fall der Pflegeversicherung ist zunächst zu betonen, daß nicht jeder Anstieg der Anzahl "versorgter" Fälle oder des insgesamt konsumierten Leistungsvolumens infolge $\mathrm{e}^{71}$ der Einführung einer Versicherung von vornherein als Moral hazard interpretiert werden darf. ${ }^{72}$ Vielmehr können solche Zuwächse z.B. auch darauf zurückzuführen sein, daß ein zuvor vorhandenes, überwiegend auf den Leistungen der Mindestsicherung basierendes Angebot qualitativ oder quantitativ unzureichend war ${ }^{73}$ oder Betroffene auf die Deckung eines zweifelsfrei vorhandenen Bedarfs verzichtet haben, um der Verarmung und/oder der vielfach als erniedrigend empfundenen Einstufung als "Sozialfall" zu entgehen. Generell gilt - das sei hier nochmals mit Nachdruck betont: Die Referenzlage zur Messung des "Moral hazard" ist nicht der versicherungslose Zustand, sondern derjenige einer Idealversicherung im oben erläuterten Sinn der vollständigen Beobachtbarkeit des Versichertenverhaltens. Dieser aber ist im Vergleich zur Ausgangslage ohne Versicherung regelmäßig mit einem allokativ unbedenklichen Anstieg der Schadenssumme verbunden.

Andererseits gilt auch für die Pflegeversicherung, daß das relevante Verhalten der Versicherten nicht ohne weiteres zum Zweck einer entsprechenden Beitragsgestaltung erfaßt werden kann. Wenn Möglichkeiten zur Beeinflussung der Pflegewahrscheinlichkeit im Sinne von Moral hazard ex ante gegeben sind, werden sie in der Sphäre der persönlichen Lebensführung angesiedelt sein, die für den Versicherer kaum zugänglich ist und erst recht nicht fortlaufend überwacht werden kann. In Hinblick

\footnotetext{
${ }^{71}$ Nebenbei bemerkt: Ein Leistungsanstieg nach Einführung der Versicherung kann selbstverständlich auch durch äußere, etwa demographische Faktoren mitbedingt sein, die ihre Wirkung auch ohne Versicherung entfaltet hätten.

${ }^{n}$ Dies tut implizit z.B. Dirk Meyer (1989), S. 303.

${ }^{73}$ F. Schulz-Nieswandt (1989), S. 181, spricht in bezug auf die Situation in der Bundesrepublik vom "Angebotsrationierungseffekt der dominierenden Sozialhilfefinanzierung".
} 
auf den ex post-Typus des Moral hazard sind die entscheidenden Fragen, wie präzise der bestimmte Leistungen auslösende Tatbestand der Pflegebedürftigkeit formuliert und wie zuverlässig sein Vorliegen ermittelt werden kann.

Im einzelnen kommen die folgenden Verhaltensweisen als mögliche Ausprägungen des Moral hazard in bezug auf eine Pflegeversicherung in Betracht: ${ }^{74}$

a) die fahrlässige Herbeiführung des Pflegefalls

b) die Vortäuschung des Pflegefalls

c) die Inanspruchnahme unnötig vieler Leistungen

d) die Inanspruchnahme unnötig aufwendiger Leistungsarten

e) die Inanspruchnahme unnötig teurer Leistungen bei gegebener Art und Menge

Bei a) und b) handelt es sich um Verhaltensweisen vor dem (bzw. ohne) Pflegefall, die übrigen Spielarten sind ex post. Urheber des Moral hazard können neben den Versicherten selbst aufgrund der sozialen Komponente des Pflegefalls sowie der mitunter eingeschränkten Autonomie der Pflegebedürftigen evtl. auch deren Angehörige sein. ${ }^{75}$ Zunächst aber seien die fünf Varianten für den Fall durchgespielt, daß der Moral hazard vom Versicherungsnehmer in eigener Person ausgeht.

\subsubsection{Moral hazard seitens der Versicherten selbst}

ad a): Dieser "klassische Fall" von Moral hazard kann, zumindest in bezug auf den medizinischen Kern der Pflegebedürftigkeit, ausgeschlossen werden. Für ein solches Verhalten wäre es nämlich "erforderlich, daß die Faktoren, die zu Pflegebedürftigkeit führen, in ihrem Einfluß und Zusammenwirken dem Pflegebedürftigen bekannt wären". ${ }^{76}$ Es ist schon mehrfach auf das Doppelgesicht des Zusammenhangs zwischen

\footnotetext{
${ }^{74}$ Weitgehend ausgeblendet bleibt dabei die Wirkung der Versicherung auf die Leistungsanbieter, die M. Nell (1992), S. 144ff., unter der Bezeichnung "externes moralisches Risiko" ausführlich behandelt. Am Ende dieses Kapitels wird auf diesen Aspekt noch kurz eingegangen.

75 vgl. Dirk Meyer (1989), S. 303f.

${ }^{76}$ A. Prinz (1987), S. 108. Es erstaunt, daß Prinz selbst nur wenige Seiten zuvor eine "Lebensweise, die das Pflegerisiko erhöht" (S. 101) als Möglichkeit ins Spiel gebracht hat.
} 
Lebensweise und Pflegerisiko ${ }^{77}$ hingewiesen worden: Ein "gesundes Leben" erhöht kurzfristig natürlich die Chancen, ohne fremde Hilfe auszukommen, steigert gleichzeitig aber die Lebenserwartung. Wer jedoch ein hohes Alter erreicht, ist dann einem stark erhöhten Pflegerisiko ausgesetzt. ${ }^{78}$

Weiterhin gilt es für diese Variante des Moral hazard grundsätzlich zu beachten, daß der Pflegefall im allgemeinen und seine stationäre Variante im besonderen auch bei Versicherung gemeinhin als hochgradig unerwünscht angesehen wird. ${ }^{79}$ Dieser Tatbestand wirkt als "Natürliche Selbstbeteiligung" mit der Folge, daß fahrlässiges Verhalten, wenn überhaupt möglich, höchstens bei Vorliegen kräftiger Anreize attraktiv sein könnte.

$\mathrm{Zu}$ überlegen ist allenfalls, ob die nichtmedizinischen Aspekte der Bedürftigkeit ${ }^{80}$, also etwa die Wohnform oder die Anwesenheit pflegefähiger und -williger Bezugspersonen, einem vorausschauenden Verhaltenseinfluß unterliegen. So könnten etwa der rechtzeitige Bezug einer behindertengerechten Wohnung oder gar die besonders sorgfältige Pflege familiärer Beziehungen im Prinzip insoweit als "Vermeidungsaktivitäten" in bezug auf den Pflegefall angesehen werden, wie sie bei gegebener medizinischer Bedürftigkeitslage die Kosten der Pflege gering zu halten versprechen. In beiden Fällen ist der Zusammenhang zum Pflegefall aber recht vage und das Verhalten zu stark von anderweitigen Motiven bestimmt, als daß hier ein nennenswerter Einfluß der Versicherung anzunehmen wäre.

ad b): In einem engen Sinn verstanden, ist dies ein Fall von Versicherungsbetrug, dem in der Praxis ebenfalls keine große Bedeutung beizumessen sein dürfte. ${ }^{81} \mathrm{Da}$

\footnotetext{
${ }^{77}$ P. Zweifel et al. (1994), S. 66, behaupten einen - leidlich signifikanten - Einfluß von Übergewicht auf das Pflegerisiko. Ermittelt haben sie aber offenbar nur, daß Heimbewohner im Durchschnitt übergewichtiger sind als andere Menschen. Ob ein solcher Rückschluß zulässig ist, erscheint mir sehr fraglich.

${ }^{78} \mathrm{Da}$ auch das Krankheitsrisiko im hohen Alter stark ansteigt, läßt sich so eventuell auch für die Krankenversicherung argumentieren (vgl. M. Nell (1992), S. 139ff.). Die Forderung nach gestaffelten Beiträgen je nach Lebensführung läßt sich dann in der gängigen Form nicht aufrecht erhalten.

${ }^{79}$ vgl. W. Buchholz/W. Wiegard (1992), S. 452

${ }^{80}$ siehe E.-M. Dennebaum/W. Rückert (1987), S. $175 f$.

${ }^{81}$ H.-W. Sinn (1977), S. 531 ist überdies der Ansicht, diese Variante sei "vom Allokationsstandpunkt neutral zu beurteilen". Dem ist zu widersprechen: Wenn es im Umfang der Vortäuschungen zu Prämienerhöhungen kommen muß, die den Abschluß der Versicherung für ehrliche Leute unattraktiv werden
} 
Pflegeleistungen in aller Regel keinen positiven Nutzen stiften, wenn kein Bedarf daran besteht, wird sie sich auch niemand erschleichen wollen. Anders ist dies im Bereich von Sachleistungen allenfalls in Hinblick auf hauswirtschaftliche Dienste. Theoretisch vorstellbar ist zudem, daß ein großzügig bemessenes Sachleistungspaket Güter enthält, die trotz fehlender Pflegebedürftigkeit willkommen sind, da sie ohnehin konsumiert worden wären (etwa Wohnung und Essen bei Heimunterbringung). Dann würde sich der finanzielle Spielraum durch Vortäuschung des Pflegefalls vergrößern. Da dies aber nur bei der zumeist unerwünschten Heimpflege auftreten kann, ist der Fall eher abwegig. ${ }^{82}$ Zudem wäre ein solches Leistungspaket, das vom Eintritt des Versicherungsfalles unabhängige Bedürfnisse abdeckt, falsch konstruiert.

Ein klarer Anreiz zur hier diskutierten Form des Moral hazard besteht mithin nur dann, wenn die Versicherung ungebundene Geldleistungen gewährt. Freilich wird es - abgesehen von Gewissensgründen, die einem solche Verhalten entgegenstehen können - nicht jedermanns Sache sein, sich gerade auf diesem, doch eher aufwendigen und peinlichen Weg in den unberechtigten Genuß von Leistungen zu bringen.

Im übrigen verlagert sich die Betrachtung für diesen Fall auf die Frage, ob die Täuschung gelingen kann. Obliegt die Feststellung des Pflegefalls einer hinreichend kompetenten und unabhängigen Instanz, ist kaum davon auszugehen, daß dies in großem Umfang der Fall der sein wird. Dennoch bleibt hinsichtlich eines gewissen "Restrisikos" festzuhalten, daß die "Geldleistung in jedem Fall anfälliger für Mißbrauch [ist] als die Sachleistung". ${ }^{83}$

lassen, entgeht ihnen dadurch der mittels einer betrugsfreien Versicherung erzielbare Wohlfahrtsgewinn. ${ }^{82}$ Ins Pflegeheim umziehen, um die Miete zu sparen. Daß derart realitätsfremde Verhaltensspielarten überhaupt erwähnt werden, ist dem Bemühen geschuldet, gerade in der Anreizdiskussion keinen erdenklichen Aspekt auszulassen. Dieses Bestreben wiederum hängt damit zusammen, daß der Glaube an die Wirksamkeit monetärer incentives in allen Lebenslagen unter Ökonomen extrem stark ausgeprägt ist. Ein Beispiel im vorliegenden Zusammenhang liefert R. Vaubel (1983), S. 163, wenn er in einer Anfang der 80er Jahre von der Bundesregierung beschlossenen Anhebung des "Taschengeldes" für Heimbewohner um 18\% (absolut dürfte es sich allenfalls um einen Zuschlag von knapp 30 DM gehandelt haben) einen "Anreiz, sich ins Heim zu begeben", erblickt.

${ }^{83}$ So Eckart FIEDLER in einer Gesprächsrunde am 22.03.1991, siehe Bayerische Rück (1991), S. 29. 
Anders zu bewerten sind Personen, bei denen eine gewisse Beeinträchtigung gegeben ist, die aber nach herrschenden Maßstäben noch keinen wirklichen Pflegebedarf begründet. Diese Konstellation ist als Grenzfall dem nächsten Punkt zuzuordnen.

ad c) und d): In bezug auf diese Varianten ist es zunächst von entscheidender Bedeutung, ob von einem objektiv feststellbaren und in Ansprüche auf spezifische Leistungen übersetzbaren Bedarf ausgegangen wird, der sozialmedizinisch und/oder sozialpolitisch zu definieren wäre. Soweit dem so ist, kann von "Moral hazard" im Sinne eines Effizienzproblems keine Rede sein. ${ }^{84}$ Die Wahrnehmung solcher Ansprüche ist als unabdingbares Moment der Effektivität auch dann stets unbedenklich, wenn sie ohne Versicherung unterblieben wäre. Das Problem reduziert sich dann darauf, daß eine solche Bedarfsfeststellung im Sinne der Vorgaben in hinreichend leistungsspezifischer Form auch erfolgt und möglichen Täuschungsversuchen (die sich ja auch auf die Schwere der Bedürftigkeit beziehen könnten) vorgebaut wird.

Grundsätzlich erscheint eine solche Bedarfsfeststellung im Pflegebereich der Sache nach möglich. Kann es etwa im Gesundheitswesen vielfach zweifelhaft sein, ob bestimmte Therapie-Elemente oder präventiv-diagnostische Maßnahmen ${ }^{85}$ wirklich sinnvoll und nötig sind, so bestimmt sich ein Pflegebedarf nach Art und Umfang vergleichsweise eindeutig nach denjenigen alltagsnotwendigen Verrichtungen, die der Betroffene selbst nicht mehr vornehmen kann. Dennoch wird es auch hier gewisse Grauzonen und Ermessensräume geben, in deren Grenzen ein übermäßiger Leistungskonsum im Sinne von Moral hazard möglich ist.

Insoweit ist wiederum zu prüfen, unter welchen Umständen ein exzessives Ausschöpfen derartiger Spielräume im Interesse des Pflegebedürftigen liegt. Analog zu den Ausführungen unter $b$ ) ist festzustellen, daß Pflegeleistungen keinen bedarfsunabhängigen Nutzen stiften. Das gilt auch in der Mengenbetrachtung für diejenigen Leistungen, die den tatsächlichen Bedarf übersteigen und ebenso für die Hierarchie der Leistungsformen: Es ist in aller Regel nicht davon auszugehen, daß ein Bedürftiger durch die Wahl der in seinem Fall nicht zwingend erforderlichen stationären Unterbringung als

\footnotetext{
${ }^{84}$ Die bei F. Breyer/P. Zweifel (1992), S. 172 genannte Bedingung für den moral hazard ex post, $\mathrm{da} ß$ "teurere und billigere Behandlungsstrategien zur Verfügung stehen", ist dann nicht erfüllt.

${ }^{85}$ Für Beispiele vgl. etwa H.-H. Abholz (1993).
} 
der zumeist teureren Variante sein Nutzenniveau zu erhöhen vermag. ${ }^{86}$ Wiederum ist ein Anreiz zum Moral hazard also im wesentlichen auf die Fälle beschränkt, in denen Geldleistungen in Aussicht stehen.

Diese Reduktion des Problems ist allerdings nur dann ohne weiteres möglich, wenn funktionstüchtige Alternativen unterhalb der Heimschwelle existieren und aus Konsumentensicht nicht erheblich kostspieliger sind als die stationäre Pflege. Andernfalls droht ein Phänomen, das Lynn PARINGER mit der etwas sperrigen Bezeichnung "market basket moral hazard" versehen hat und wie folgt definiert: "People are encouraged to consume services that they may not need in order to acquire some that they do need". ${ }^{87}$ Die unnötig teure und mutmaßlich auch für die Betroffenen suboptimale "Paketlösung" Pflegeheim wird gewählt, weil benötigte Teilleistungen gar nicht oder nicht im Rahmen der Versicherung angeboten werden. Die Differenziertheit des Leistungsspektrums einer Pflegeversicherung im Bereich ambulanter und teilstationärer Dienste, wie sie als wichtiges Moment einer zielgerechten Gestaltung bereits gefordert wurde, erweist sich mithin gleichzeitig als Effizienzgebot.

Bemerkenswert ist dabei auch, daß PARINGERs Befund ${ }^{88}$ sich konkret auf die USA und damit auf eine versicherungslose Situation bezieht, in der - ebenso wie in Deutschland - ein Großteil der Heimkosten mangels Zahlungsfähigkeit der Pflegebedürftigen zulasten der Mindestsicherung (hier: Medicaid) anfällt. Mit der Bereitstellung nichtstationärer Leistungen kann die Pflegeversicherung in diesem Punkt also nicht nur die Entstehung von Moral hazard verhindern, sondern einen aufgrund der bloßen Mindestsicherung bereits bestehenden Fehlanreiz abstellen.

Noch in einem weiteren Fall ist es vorstellbar, daß Sachleistungen in der Form stationärer Pflege ohne unmittelbare Notwendigkeit nachgefragt werden: Ist das Angebot an Pflegeplätzen in dem Sinne knapp, daß nicht in allen Fällen akuten Bedarfs die Möglichkeit einer (sofortigen) Unterbringung besteht, so kann es sinnvoll sein, schon

\footnotetext{
${ }^{86}$ Siehe F. Schulz-Nieswandt (1989), S. 181 und die dort angegebene Literatur.

${ }^{87}$ L. Paringer (1983), S. 141

${ }^{88}$ Zum Beleg verweist die Autorin auf Untersuchungen, denenzufolge amerikanische Pflegeheime in erheblichem Umfang "fehlbelegt" sind (vgl. ebenda, S. 125 und die dort genannte Literatur). Laut K.H. Schönbach (1985), S. 40f., gilt dies auch für die Bundesrepublik. Hans Brandt (1987 a), S. 45, ist in diesem Punkt empirisch anderer Ansicht, betont aber ebenfalls, daß die Notwendigkeit der Heimpflege von der Verfügbarkeit und Leistungsfähigkeit ambulanter Dienste abhängt.
} 
bei beginnender Pflegebedürftigkeit vorsorglich einen solchen Platz einzunehmen, wenn sich die Gelegenheit dazu bietet. ${ }^{89}$ Indem ein solches Verhalten c.p. die Knappheit an Pflegebetten verschärft, kann auf diese Weise sogar eine Art "Teufelskreis" in Gang kommen. Ebenso wie der zuvor genannte Punkt gehört dieser Effekt nur im weiteren Sinn zur Problematik des Moral hazard, da er nicht durch die Existenz einer Versicherung als solche ausgelöst wird. Entscheidend ist vielmehr das zusätzliche Vorliegen bestimmter Angebotsmängel.

ad e): Wird eine dem Bedarf nach unstrittige Pflegeleistung im Rahmen eines Sachleistungskonzepts zur Gänze von der Versicherung bezahlt, so ist ihr Preis für den Bedürftigen naturgemäß ohne Belang. Es ist allerdings nicht anzunehmen, daß er sich einen besonders teuren Anbieter ohne weiteres gezielt aussuchen kann. Sein Wahlrecht wird vielmehr auf solche Leistungserbringer beschränkt sein, die in einen mit dem Versicherungsträger ausgehandelten Preis- und Leistungsstandard eingebunden sind. Effizienzfragen in Zusammenhang mit einem solchen Verhandlungsproze ${ }^{90}$ gehören nicht zum Komplex des Moral hazard im hier behandelten Sinn.

In Geldform fixierte Leistungen sind übrigens auch in diesem Punkt nur auf den ersten Blick unproblematisch: Zwar belassen sie die "effizienzsichernde Kontrolle durch die Nachfrager"91 beim Leistungskonsumenten selbst; ob dieser zur Ausübung einer solchen Funktion aber besser imstande ist als die Versicherung, ist zweifelhaft.

Die bisherigen Überlegungen haben gezeigt, daß die Existenz einer Pflegeversicherung auf seiten der Versicherten selbst nur bedingt die Gefahr birgt, Fehlanreize im Sinne eines Moral hazard auszulösen. Dies gilt insbesondere für Sachleistungen, Geldleistungen sind in dieser Hinsicht problematischer. Neben dem Anreiz sind aber die Möglichkeiten zum Moral hazard recht eingeschränkt: Ist es ex ante praktisch unmöglich, das Schadensrisiko gezielt zu beeinflussen, so ist das Verhalten der Versi-

\footnotetext{
${ }^{89}$ vgl. N.J.H.W. van Weert (1991), S. 185

${ }^{90}$ Siehe dazu M. Nell (1992), S. 159ff.: Zwar würden "auch bei einer Preisbildung über Verhandlungen (...) tendenziell die relativen Knappheiten berücksichtigt", eine Unterlegenheit dieses Verfahrens gegenüber der Marktpreisbildung ergebe sich aber aus dem "vergleichsweise sehr hohen Zeitbedarf". Nell hat dabei den Bereich der medizinischen Leistungen im Auge. Da Pflegeleistungen weniger stark differenziert und weniger "rationalisierungsfähig" sind als z.B. ärztliche, erscheinen die operativen Mängel der Verhandlungspreisbildung dort weniger gravierend.
}

${ }^{91}$ Dirk Meyer (1989), S. 304 
cherten ex post insoweit steuerbar, wie die Bedarfsfeststellung und mit ihr die Leistungsgewährung nach objektiven Maßstäben vorgenommen werden kann. Wichtig ist mithin ein Indikationsverfahren, das in den Händen einer Instanz liegt, die weder ein eigenes Interesse an einer Leistungsausweitung hat, noch als unkritischer Agent der Anspruchsberechtigten fungiert. ${ }^{92}$

\subsubsection{Moral hazard seitens der Angehörigen Pflegeversicherter}

\subsubsection{1 $\quad$ Einleitung}

Im folgenden ist zu prüfen, inwieweit der bisherige Befund revidiert werden muß, wenn auch die Möglichkeit des Moral hazard durch Angehörige Bedürftiger in Betracht gezogen wird. Die Untersuchung soll wieder anhand der fünf genannten Varianten erfolgen, von denen allerdings die letzte keiner neuerlichen Behandlung bedarf.

ad a): Die Herbeiführung des - medizinisch verstandenen - Pflegefalls ist allenfalls als schwerkrimineller $\mathrm{Akt}^{93}$ vorstellbar und braucht hier nicht erörtert zu werden. Eine evtl. "Herbeiführung" im weiteren Sinn der nichtmedizinischen Aspekte ist in die Behandlung der folgenden Punkte einbegriffen.

ad b): Eine von den Angehörigen unternommene Vortäuschung des Pflegefalls bedarf, um Erfolg haben zu können, der Mitwirkung des Betroffenen selbst. Diese wird er verweigern, wenn das Manöver darauf abzielt, ihn als unliebsamen Mitbewohner loszuwerden. Somit ist ein Anreiz wie oben nur bei Aussicht auf Geldleistungen gegeben. Eine Haushaltshilfe, die auch ohne wirkliche Beeinträchtigung als nützlich empfunden werden könnte, wird nicht gewährt werden, wenn ihr möglicher Empfänger mit "Gesunden" zusammenlebt. Ist dies nicht der Fall, besteht seitens der Angehörigen auch kein Interesse.

\footnotetext{
${ }^{2}$ Im Gesundheitswesen ist diese Bedingung typischerweise nicht erfüllt, da der Arzt den Bedarf feststellt oder zumindest spezifiziert, den er selbst zu decken berufen ist (vgl. F. Breyer/P. Zweifel (1992), S. 225ff.). Hierin wird eine zentrale Ursache für die (möglicherweise) übermäßige Inanspruchnahme medizinischer Leistungen gesehen. Eine Lösung in Analogie zur hier für die Pflege vorgeschlagenen scheitert an der ungleich größeren Fallzahl im Vergleich zum Leistungsvolumen.

93 L.H. und C. Schreiber (1993), S. 148, nennen ein ungefähr passendes Beispiel: "Ein altes, zuckerkrankes Ehepaar wurde immer dann falsch und unregelmäßig von den Kindern ernährt, wenn diese Urlaubsreisen antreten (...) wollten. " Ein Krankenhausaufenthalt der Eltern war die planmäßige Folge.
} 
ad c): Für die Beurteilung des nur bei den ambulanten Leistungen denkbaren übermäßigen Konsums der Menge nach ist wiederum die bereits diskutierte Abgrenzung des Moral hazard von sozialpolitisch erwünschten und damit auch allokativ unbedenklichen Effekten zentral. Eine Entlastung der privat Pflegenden ist schon deswegen Teil des Zielkatalogs, weil sie eine hinreichende Qualität der häuslichen Pflege sicherstellen hilft. Ferner ist sie auch selbst als "externer Effekt" der Versicherung zugunsten der Pflegekräfte ausdrücklich erwünscht. Somit gilt auch hier, daß die verstärkte Inanspruchmahme ambulanter Dienste als Folge der Versicherung nicht per se als Effizienzproblem zu kennzeichnen ist. Wie oben dargelegt, kann sie sogar umgekehrt als Zeichen verbesserter Effizienz gelten, sofern eine Substitution von Heimpflege stattfindet. Innerhalb des Bereichs ambulanter Dienste wird die Möglichkeit eines Moral hazard daher nicht weiter betrachtet.

Gleichwohl stellt sich grundsätzlich wieder die Frage, wie weit genau eine solche Entlastung gehen darf, ohne den Tatbestand des Moral hazard zu erfüllen. Sie kulminiert im Fall der völligen Abwälzung der Pflegelast durch die Heimeinweisung, die denn auch teils unter dem Schlagwort der "Abschiebung" in der Bundesrepublik am heftigsten diskutiert worden ist. Dieser Aspekt eines möglichen Moral hazard wird daher im folgenden ausführlicher erörtert.

\subsubsection{Die vermutete "Abschiebung" ins Pflegeheim als Kernproblem}

Vorab ist zu klären, unter welcher Voraussetzung sich ein Moral hazard-Problem an diesem Punkt überhaupt stellt: Es muß im Spektrum der auftretenden Fälle einen Bereich geben, in dem eine reale Möglichkeit besteht, zwischen der stationären Lösung und einem auf das Engagement von Angehörigen gestützten Verbleib des Pflegebedürftigen in der häuslichen Umgebung zu wählen. Nach "oben" begrenzt wird dieser kritische Bereich auf einer gedachten Skala der Fallschwere durch den Punkt, an dem das private Umfeld mit der Bewältigung des Pflegefalls objektiv überfordert ist. Die "untere" Grenze hingegen ist durch die jeweils gültigen Versicherungsbedingungen hinsichtlich des Eintritts in ein Pflegeheim bestimmt. Ferner ist wiederum von Belang, wie gut das Vorliegen dieser Bedingungen im Einzelfall überprüft werden kann und wird.

Über die quantitative Bedeutung des so definierten Fallsegments können folglich keine allgemeinen Aussagen getroffen werden. Wichtig ist aber die Feststellung, daß eine 
Option in Hinblick auf die Pflegeform bei weitem nicht in allen Fällen gegeben und das Ausmaß ihrer Relevanz durch die konkrete Ausgestaltung einer Pflegeversicherung grundsätzlich beeinflußbar ist. Daß es aber unter allen Umständen überhaupt Fälle gibt, in denen Angehörige über die Pflegeform entscheiden können, erscheint realistisch und ist im folgenden durchweg unterstellt.

Angehörige - so die hier näher zu untersuchende Vorstellung - können dann bei Existenz einer Versicherung einen kostenlosen Entlastungseffekt erzielen, wenn sie eine Heimeinweisung vorzeitig, das heißt schon dann herbeiführen, wenn sie durchaus noch selbst zur Bewältigung der Pflege imstande wären. ${ }^{94}$ Die Gefahr bzw. die Vermeidung eines solchen Effekts gilt als wesentliches Kriterium nicht nur für die Effizienz einer Versicherungslösung, sondern auch für deren Effektivität: Heimpflege, so lautet das Argument, kann nur in schweren Notfällen als bedarfsgerecht angesehen werden, ansonsten läuft sie den typischerweise anzunehmenden Präferenzen zugunsten einer privaten und vertrauten Wohnform zuwider. Da ein solches Bedürfnis als elementar einzustufen ist, ist seine Mißachtung "inhuman". "In der gesamten sozialpolitischen Diskussion um das Risiko Pflegebedürftigkeit und seine Absicherung gibt es kaum einen Punkt, der in solch hohem Maße allgemeine Zustimmung (...) findet wie die Forderung nach einem Vorrang für häusliche Pflege. "195

So plausibel und gängig eine derartige Argumentation ist, so unklar bleibt ihre Fundierung und damit auch die Reichweite und die Implikationen ihrer Gültigkeit. Zu klären gilt es zunächst, inwieweit von einer generellen Verpflichtung der Angehörigen zur eigenhändigen Übernahme von Pflegediensten ausgegangen werden kann. Besteht eine solche Pflicht, so sind die Angehörigen praktisch als mitversichert zu betrachten: Die Tatsache, daß das potentiell pflegebedürftige Familienmitglied den Schutz einer Pflegesicherung genießt, eröffnet ihnen selbst die Möglichkeit, sich ihrer Pflegepflicht unter Umständen zu entledigen. Falls aber eine solche Pflicht nicht feststellbar und im übrigen sichergestellt ist, daß die benötigte Pflege letztlich auch ohne explizite Pflegeversicherungerfolgt ${ }^{96}$, ist der Behauptung vom "familiären" Moral hazard in diesem Sinn die Basis entzogen. Der mit der Einführung einer Pflegeversicherung für den stationären Fall verbundene zusätzliche Schutz der Angehörigen

\footnotetext{
${ }^{94}$ vgl. z.B. Frankfurter Institut (1991), S. 4

${ }^{95}$ R. Thiede (1990), S. 180

${ }^{96}$ nämlich im Wege der Mindestsicherung
} 
reduziert sich dann darauf, daß sie mit geringerer Wahrscheinlichkeit zur Zahlung von Unterhalt herangezogen oder in ihrer Eigenschaft als mögliche Erben begünstigt werden. Welche Bedeutung der Moral hazard in diesem begrenzten Zusammenhang haben kann, wird weiter unten zu prüfen sein.

\subsubsection{Zur juristischen Frage der familialen Pflegepflicht}

Die folgenden Überlegungen beschränken sich auf juristische Aspekte des Eltern-KindVerhältnisses, da es hinsichtlich der Fragestellung überragende Bedeutung hat: Ist bei weniger direkten Verwandtschaftsbeziehungen ohnehin nicht von der Existenz einer Pflegepflicht auszugehen, so ist bei zusammenlebenden Eheleuten zu unterstellen, $\mathrm{da} ß$ die Einweisung eines Partners in ein Pflegeheim nur in wirklichen Notfällen in Betracht gezogen wird. Diese Annahme wird durch Untersuchungen bestätigt. ${ }^{97}$ Als Beleg kann ferner gelten, daß in der Bundesrepublik nur 7\% aller Heimbewohner verheiratet sind ${ }^{98}$, während im Bereich der häuslichen Betreuung Bedürftiger der Ehepartner (bzw. vor allem die Ehepartnerin) am häufigsten als Hauptpflegeperson fungiert. ${ }^{99}$

"Eine Regel (..), derzufolge Eltern von ihren Kindern Unterhalt in Gestalt von Naturalpflege beanspruchen könnten, enthält das BGB nicht. ${ }^{100} \mathrm{Da}$ das Unterhaltsrecht ausdrücklich auf Geldleistungen abstellt und andere Formen nur für bestimmte Fälle vorsieht, in denen der Unterhaltspflichtige selbst dies wünscht, ( $\$ 1612$ BGB), wäre eine Pflegepflicht allenfalls aus $§ 1618 \mathrm{a}$ ableitbar, der folgenden Wortlaut hat: "Eltern und Kinder sind einander Beistand und Rücksicht schuldig." In einer ausführlichen Analyse dieser Bestimmung und ihrer Reichweite gelangt KNÖPFEL unter Verweis auf den Grundsatz der Verhältnismäßigkeit zu der Einschätzung, daß

${ }^{97}$ siehe H. Braun (1987), S. 24
${ }^{98}$ W. Krug/G. Reh (1992), S. 31
${ }^{99}$ Von allen in Privathaushalten lebenden Pflegebedürftigen (einschließlich Kinder und junge Menschen)
werden $37 \%$ hauptsächlich vom Lebenspartner versorgt. Dieser ist in zwei Dritteln der Fälle weiblich.
Der Anteil der Töchter und Schwiegertöchter unter den Hauptpflegepersonen beträgt $35 \%$, während
Söhne nur in drei von Hundert Fällen genannt werden (siehe U. Schneekloth/P. Potthoff (1993), S. 128).

100 A. Hänlein (1992), S. 113 
"die dauernde Aufnahme gebrechlicher Eltern in den Hausstand des Kindes" die dort angelegte Verpflichtung übersteigt. ${ }^{101}$

Anders als etwa eine "kurzfristige Betreuung in Notfällen"102 gehöre Dauerpflege nämlich nicht zu den Hilfeleistungen, die nach "allgemeiner Übung" unter Angehörigen erwartet werden könnten. An diesem allgemein Üblichen aber habe sich die Auslegung des Paragraphen auszurichten, dessen Funktion nicht darin bestehe, spezielle Pflichten zu generieren, sondern gesellschaftlich Anerkanntes nachzuvollziehen und zu bekräftigen. Damit korrespondiert, "daß allein aus § 1618a ein klagbarer Anspruch [ohnehin, S.W.] nicht herzuleiten ist". ${ }^{103}$ "Erzwungener Beistand" nämlich, und dies gilt im Pflegefall sicher in besonderem Maße, würde die familiären Beziehungen, die die Bestimmung zu festigen bezweckt, "im ganzen mehr belasten als fördern". ${ }^{104}$

Dieser für die Bundesrepublik gültige Befund läßt sich offenbar auf andere industrialisierte Gesellschaften problemlos übertragen, da lediglich in der Schweiz eine dem genannten Paragraphen vergleichbare, nirgends jedoch eine weitergehende Vorschrift existiert. ${ }^{105}$ HÄNLEIN verweist auf schwächer ausgebildete Unterhaltspflichten in Skandinavien, England und den Niederlanden. Speziell in Dänemark fehle eine Unterhaltspflicht der Kinder zugunsten ihrer Eltern zur Gänze, "so daß eine Heranziehung für Pflegeaufwand insbesondere alter Menschen nicht möglich ist". ${ }^{106}$

Mithin ist festzuhalten: Die Vorrangstellung häuslicher Pflege kann nach herrschender Rechtslage nicht so verstanden werden, daß nahe Angehörige grundsätzlich im Rahmen ihrer Möglichkeiten als Pflegekräfte bereitstünden. Vielmehr kommt eine Lösung des Pflegeproblems innerhalb der Familie ohnehin nur dann in Betracht, wenn die Kinder des Bedürftigen oder andere Verwandte sich freiwillig zur Übernahme der Verantwortung bereitfinden. Folglich bedürfen Angehörige auch nicht der Existenz einer Pflegeversicherung, um sich der mit eigenhändiger Pflege verbundenen Belastung zu entziehen.

\footnotetext{
${ }^{101}$ G. Knöpfel (1985), S. 562

102 ebd., S. 564

${ }^{103}$ ebd., S. 560

${ }^{104}$ ebd., S. 561

${ }^{105}$ siehe ebd., S. 554

${ }^{106}$ A. Hänlein (1992), S. 4
} 
Allenfalls im Umfang ihrer Unterhaltspflicht nach Maßgabe des eigenen Einkommens oder hinsichtlich ihres Erbinteresses erwächst den Angehörigen aus der Versicherung ein Vorteil, der im Sinne von Moral hazard prinzipiell ausnutzbar wäre. Die mögliche Bedeutung dieses Aspekts soll im folgenden näher betrachtet werden. Zunächst aber sei kurz auf die Frage eingegangen, wie sich die aus juristischer Sicht soeben verneinte Pflegepflicht in ökonomischer Perspektive darstellt.

\subsubsection{Zur theoretischen Spannung zwischen Nutzenkalkül und Familienidyll}

Gerade in ökonomischen Beiträgen zur Pflegedebatte wird die Existenz einer vorrangigen Pflegepflicht der Angehörigen suggeriert und zur Ableitung von Moral hazardGefahren verwandt. ${ }^{107}$ Auch abgesehen davon, daß er sich juristisch nicht stützen läßt, ist ein solcher Ansatz nicht unproblematisch:

In der Welt der ökonomischen Theorie herrscht das Denkmuster der individuellen Nutzenmaximierung vor. Ein aktives Interesse am Wohlergehen anderer wird zwar unter dem Titel Altruismus als mögliches Phänomen zur Kenntnis genommen und üblicherweise als "höhere Form des Egoismus"108 - in die Modellwelt integriert. Keineswegs aber hat es den gleichen konstitutiven und quasi natürlichen Rang wie die "selfishness", deren moralische Unbedenklichkeit im übrigen seit Adam Smith für Ökonomen grundsätzlich außer Frage steht. ${ }^{109}$

Die Erwartung, Angehörige hätten im Pflegefall nach Kräften durch persönlichen Arbeitseinsatz (und nicht etwa nur mit affektiven, am Markt grundsätzlich nicht erhältlichen Leistungen) unentgeltlich einzuspringen, paßt zunächst schlecht in ein solches Konzept und kollidiert im übrigen auch mit der Idee der Arbeitsteiligkeit als

\footnotetext{
${ }^{107}$ So spricht beispielsweise Dirk Meyer (1989), S. 303f. von "Verwandte[n], die schon bei geringer persönlicher Belastung die Verantwortung auf das Kollektiv abwälzen." Es ist nicht ganz klar, welche Fälle Meyer hier vor Augen hat. Wenn Angehörige sich lediglich von ambulanten Diensten unterstützen lassen, kann kaum von abgewälzter Verantwortung die Rede sein; meint er damit hingegen die Heimeinweisung, so wird sich diese in Fällen "geringer Belastung" kaum erwirken lassen.

${ }^{108}$ P. Zweifel et al. (1994), S. 51

109 vgl. Gary S. Becker (1981), S. 12: "Unfortunately, however, we do not have an analysis of the consequences of altruism (...) that is as the analysis of selfishness in market transactions developped during the last two centuries."
} 
Voraussetzung des ökonomischen Fortschritts. Technisch betrachtet wird ein pflegender Angehöriger, der diese Tätigkeit weder frei gewählt noch gelernt hat, mit suboptimaler Produktivität arbeiten. Zudem werden bei der Heimpflege, selbst wenn sie nicht quasiindustriell verrichtet wird, in Teilbereichen Skaleneffekte wirksam. Dies gilt etwa für Pflegebedürftige, die aus Sicherheitsgründen beaufsichtigt werden müssen.

Insoweit ist das gängige Argument, bei familiengestützter häuslicher Pflege seien neben dem Vorzug in menschlicher Hinsicht auch die "Kosten niedriger als bei einer Unterbringung im Heim"110, zumindest in genereller Form unzutreffend. ${ }^{11}$ Es gilt allenfalls in einer Betrachtung, die sich auf die in der Sozialproduktrechnung ausgewiesenen Kosten beschränkt. ${ }^{112}$ Müßten die privaten Pflegekräfte marktgerecht entlohnt werden, so würden die Kosten pro Fall die der stationären Unterbringung weit übersteigen. Von daher ist der Vorrang häuslicher Pflege, die als Referenzsituation zur Herleitung des Moral hazard dient, selbst nicht wirklich ökonomisch begründbar, sondern hat den Status einer exogenen Wertvorgabe. Sie läuft darauf hinaus, daß die Familienpflege als "Produkt" besonderer Qualität eingestuft wird, das durch professionelle Dienste nicht in gleichwertiger Form erzeugt werden kann.

Nun kann aber keineswegs unbesehen vorausgesetzt werden, da - auf den eigenen Nutzen bedachte - Angehörige dieses Produkt oder auch bloß eine "technisch" angemessene Pflege auch wirklich bereitstellen. Es obliegt dem Staat, die Einhaltung derartiger Spielregeln mit Hilfe von Sanktionsdrohungen durchzusetzen und zu überwachen. Dies aber ist im Fall der familiären Pflege praktisch nur sehr eingeschränkt möglich. ${ }^{113}$ Wird dem Pflegebedürftigen die im Sinne des Sicherungsziels

\footnotetext{
${ }^{110}$ Wissenschaftlicher Beirat beim BMF (1990), S. 4. Bemerkenswert ist an dieser Textstelle auch die implizite Gleichsetzung von familiärer Betreuung und "menschliche(r) Zuneigung" (siehe ebd.) Sie schließt eine emotionale Qualität der Pflegebeziehung im Heim ebenso aus wie deren Fehlen im häuslichen Zusammenhang.

${ }^{111}$ Auch W. Buchholz/W. Wiegard (1992), S. 453, sind hier skeptisch.

112 vgl. dazu auch L. Paringer (1983), S. 127

113 "Die Ermessung der Leistung in der Familie ist schwer. Sowohl Umfang wie Qualität der Leistungen sind kaum kontrollierbar" (Bundesminister für Jugend, Familie, Frauen und Gesundheit (1986), S. 176). Die dort vorgeschlagene Verpflichtung der Angehörigen zur Teilnahme an Beratungsgesprächen und Qualifikationsmaßnahmen könnte wohl nur bedingt Abhilfe schaffen.
} 
erforderliche angemessene Betreuung von den Angehörigen versagt, so wird dies nur in extremen Einzelfällen bemerkt, nachgewiesen und sanktioniert werden können. ${ }^{114}$

Mithin kann der Staat die Familienpflege als vorrangigen Wert nicht einfach kraft seiner demokratisch legitimierten Definitionsmacht setzen, sondern muß als Voraussetzung eine gewisse Bereitschaft der Betroffenen unterstellen, ihrer Verantwortung auch freiwillig gerecht $\mathrm{zu}$ werden. Dem entspricht der juristische Befund, daß eine Erzwingung privater Pflegeleistungen gesetzlich nicht vorgesehen ist. Inwieweit davon abgesehen das Vertrauen in die Familie als Pflegeinstanz gerechtfertigt ist ${ }^{115}$, soll hier nicht diskutiert werden. Wichtig ist allein festzuhalten, daß diese aus staatlicher Sicht notwendige Unterstellung das Vorhandensein anderer als nur finanzieller Handlungsmotive impliziert.

Auf dieser Basis kann dann aber auch nicht ohne weiteres davon ausgegangen werden, daß die Angehörigen in der Pflegefrage in der modellüblichen Weise und vielleicht sogar hochelastisch auf finanzielle Anreize reagieren. Wenn also Ökonomen einerseits die besondere Qualität der Pflege durch die Familie betonen und andererseits die Gefahr von Moral hazard als Folge einer Pflegeversicherung beschwören, so tut sich zwar kein Widerspruch, aber doch zumindest ein Spannungsverhältnis auf zwischen widerstreitenden Verhaltensannahmen, die gleichzeitig verwendet werden.

Ein direkter Widerspruch liegt insofern nicht vor, als sich finanzielle und "altruistische" Motive im Prinzip als addierbar denken lassen. ${ }^{116}$ Dann aber stellt sich in Hinblick auf die Abschätzung realer Moral hazard-Gefahren nicht nur die Frage der Gewichtung, sondern es ist auch zweifelhaft, ob der "Nutzen aus der altruistischen Handlung "117 tatsächlich von der finanziellen Komponente unabhängig ist. Schließlich ist die Handlung nicht mehr ohne weiteres altruistisch, wenn sie dem Akteur auch finanzielle Vorteile verschafft. Auch dort, wo die Existenz anderer als finanzieller Motive bei

\footnotetext{
114 "...werden nur wenige Fälle angezeigt, weil sich betagte Menschen schämen, auf ihr Mißgeschick, auf ihre unmenschlichen, oftmals nahen Angehörigen hinweisen zu müssen" (L.H. Schreiber/C. Schreiber (1993), S. 147).

${ }^{115}$ Sicher nicht in allen Fällen. Für einen Überblick über den empirischen Kenntnisstand in bezug auf die Mißhandlung Pflegebedürftiger durch Angehörige siehe z.B. M. Dieck (1987).

${ }^{116}$ So gehen P. Zweifel et al. (1994), S. 53, vor. Auch die Modellskizze im hier folgenden Abschnitt arbeitet mit dieser Unterstellung.

117 ebenda
} 
der Modellbildung berücksichtigt wird, bleibt also fraglich, ob dies in einer der Wirklichkeit angemessenen Form geschieht.

Ein markantes Beispiel für die hier problematisierte Sicht liefert PAULY mit seiner Figur des "strategischen Erblassers": ${ }^{118}$ Einerseits will dieser unter allen Umständen von seinen Angehörigen statt von Dritten gepflegt werden, andererseits glaubt er sie nur dadurch zum erwünschten Verhalten bewegen zu können, daß er unversichert bleibt. Nur der drohende Verzehr des Erbes durch die Pflegekosten werde die Verwandten davon abhalten, sich des bedürftigen Angehörigen zu entledigen. Das in diesem Kalkül zutage tretende Mißtrauen verträgt sich nur schlecht mit dem Zutrauen, das erst den unbedingten Wunsch nach Familienpflege plausibel macht. ${ }^{119}$

Kurz und plakativ formuliert lautet die hier verfolgte Gegenthese wie folgt: Ethisch zur Hauspflege motivierte Angehörige werden eine Heimeinweisung auch dann nicht betreiben, wenn ihnen dadurch dank einer Versicherung keine Kosten entstehen; von denjenigen hingegen, für die ein solcher finanzieller Anreiz ausschlaggebend ist, kann eine bedarfsgerechte (oder gar liebevolle und deshalb der Betreuung im Heim überlegene) Pflege vielfach ohnehin nicht erwartet werden.

\subsubsection{Das Entscheidungskalkül}

Auf dem Hintergrund der bisherigen Überlegungen soll nunmehr versucht werden, die Relevanz des Moral hazard seitens der Angehörigen anhand einer schematischen Darstellung ihres Entscheidungskalküls auszuloten:

In einer Ausgangssituation ohne explizite Pflegesicherung sieht sich die Familie insoweit mit Kosten einer Heimeinweisung konfrontiert, wie

entweder der Bedürftige selbst die Pflegekosten trägt und dabei das potentielle Erbe der Angehörigen verzehrt oder

\footnotetext{
${ }^{118}$ siehe M.V. Pauly (1990), S. $162 \mathrm{ff}$.

${ }^{119}$ Mögen diese widerstreitenden Einstellungen subjektiv immerhin dann vereinbar erscheinen, wenn die Aussicht, in ein Pflegeheim ziehen zu müssen, stark negativ besetzt ist, so erscheint die marginaltheoretische Anwendung des PAULY-Ansatzes durch P. Zweifel und W. Strüwe (1993), S. 2, noch weniger plausibel: Dort nämlich ist impliziert, daß der Pflegebedürftige auch unterhalb der Heimschwelle jegliche externe Unterstützung seiner Angehörigen bei der Pflege mißbilligt.
} 
- $\quad$ eine Mindestsicherung im Sinne der Sozialhilfe eintritt, deren Kosten bei den Angehörigen nach Maßgabe ihres Einkommens eingefordert werden. ${ }^{120}$

Im ersten Fall handelt es sich also um den Entgang eines für die Zukunft erhofften, letztlich ungewissen ${ }^{121}$ Einkommens, im zweiten ist immerhin sichergestellt, daß die eingeforderte Kostenbeteiligung keine existenzbedrohende Höhe erreicht. ${ }^{122}$

Wird unter diesen Umständen von einer Heimeinweisung abgesehen, so läßt sich folgern, daß ihr Träger die mit der häuslichen Pflege verbundene Belastung (LP) niedriger bewertet als den Nutzen des fallweise skizzierten finanziellen Vorteils (V). Wenn die Pflegeperson alternativ eine Erwerbstätigkeit aufnehmen könnte ${ }^{123}$, ist ein solches Ergebnis zunächst kaum zu erwarten. Insbesondere hinsichtlich der Arbeitszeit einschließlich mehr oder weniger pausenloser Rufbereitschaft ${ }^{124}$ ist die Betreuung eines schwer Pflegebedürftigen ${ }^{125}$ einer jeden regulären Berufstätigkeit (deren Belastungswirkung mit LE gekennzeichnet sei) klar unterlegen. ${ }^{126}$ Gleichzeitig wird das Erwerbseinkommen und damit sein Nutzen (E) in der Regel höher sein als der an das Sozialamt abzuführende Heimkostenbeitrag bzw. der - um einen Unsi-

\footnotetext{
${ }^{120}$ Siehe ausführlich zum Komplex der hier geltenden juristischen Bestimmungen die Studie von A. Hänlein (1992).

${ }^{121}$ Neben einer evtl. drohenden Enterbung besteht Ungewißheit darüber, ob man die Erblasser überleben wird sowie hinsichtlich der Gefahr, daß die Heimeinweisung in einem späteren Stadium unvermeidlich wird.

12 siehe im einzelnen A. Hänlein (1992), Kapitel 5

${ }^{123}$ Diese Annahme mag "in der Zeit hoher Arbeitslosigkeit, insbesondere von Frauen" nicht immer gerechtfertigt sein (Bundesministerium für Jugend, Familie Frauen und Gesundheit (1986) S. 155). Eine solche Argumentation verkennt aber, daß Arbeitslose sich für die Wiederaufnahme einer Erwerbstätigkeit jederzeit bereitzuhalten haben. Diese Bereitschaft ist mit einer einmal eingegangenen Pflegeverantwortung im allgemeinen unvereinbar.
}

124 "Ein hoher Anteil der Helfenden ist sowohl am Tage als auch in der Nacht voll beansprucht" (ebenda). "Hauptpflegepersonen von Pflegebedürftigen stehen zu $79 \%$ (...) praktisch täglich rund um die Uhr zur Verfügung. $91 \%$ der Hauptpflegepersonen (...) fühlen sich dann auch eher stark oder sogar sehr stark belastet" (Infratest (1993), S. 8).

${ }^{125} \mathrm{Da}$ ein schwerer Pflegefall im medizinischen Sinn vorliegt, darf unterstellt werden, da andernfalls eine Heimeinweisung nicht erwirkt werden könnte.

126 Über $40 \%$ der innerfamiliär tätigen Pflegekräfte führen eigene gesundheitliche Beeinträchtigungen auf die damit verbundenen Belastungen zurück (vgl. Braun 1987), S. 18f.). 
cherheitsfaktor bereinigte - Gegenwartswert des erhofften Erbteils, soweit es für die Pflege verbraucht zu werden droht. Es wird also in der Regel gelten:127

(1) LP $>$ LE und $E>V$ oder zumindest

(1a) $L P+E>L E+V$ bzw. V - LP $<$ E - LE,

d.h. der Nettonutzen, den eine Erwerbsarbeit verspricht, übersteigt den der Pflegetätigkeit. ${ }^{128}$ Folglich kann die Entscheidung gegen das Pflegeheim, die unter der Bedingung

(2) V - LP > E - LE

zustandekommt, nur darauf beruhen, daß entweder die privaten "Kosten" LP durch nachlässige oder eingeschränkte Pflege unter das normale Niveau gesenkt werden oder der "altruistische" Wunsch in das Kalkül eingeht, dem bedürftigen Angehörigen die Einweisung zu ersparen. Der von seiner Erfüllung ausgehende Nutzen ${ }^{129}$ sei mit $\mathrm{X}$ bezeichnet. Die Bedingung für die Wahl der häuslichen Pflege lautet dann:

(2a) $\mathrm{V}-\mathrm{LP}+\mathrm{X}>\mathrm{E}-$ LE mit $\mathrm{X}>0$.

${ }^{127}$ Dieses mit Absicht sehr anspruchslos gehaltene Modell kennt keine Zeitdimension. Bei einem längerfristigen Kalkül wäre vielfach zu berücksichtigen, daß die i.d.R. weibliche Pflegeperson mit der Entscheidung zu einem Zeitpunkt konfrontiert wird, "in dem sie nach Beendigung ihrer Mutterpflichten nun die letzte Chance hat, wieder in ihren Beruf zurückzukehren" (BMJFFG (1986), S. 92). Damit sind also durch die Entscheidung für die Hauspflege u.U. auch die Erwerbschancen für die Zeit nach Abschluß der Pflegetätigkeit beeinträchtigt.

${ }^{128}$ Das dürfte typischerweise auch in dem oben nicht berücksichtigten Fall zutreffen, daß der Pflegebedürftige die Pflegeperson aus seinem Alterseinkommen quasi entlohnt. Liegt schon das gesamte Einkommen im normalen Rentenfall deutlich unter dem Niveau der Erwerbseinkommen, so sind davon zunächst die verbleibenden Lebenshaltungskosten sowie evtl. auch die Sachkosten der Pflege in Abzug zu bringen. Der dann noch verfügbare Rest wird zumeist gering sein.

${ }^{129}$ Zur oftmals entscheidenden Rolle der "Dankbarkeit" als "psychischer Gratifikation" siehe H. Braun (1987), S. 25. 
Kommt es nun zur Einführung einer Versicherung gegen die Pflegeheimkosten und damit zum Wegfall ${ }^{130}$ von $\mathrm{V}$, so wird dies

- $\quad$ im Fall des "gedrückten" LP bei $X=0$ den Übergang zur Heimpflege bewirken. Das ist im Sinne des Sicherungsziels auch erwünscht, da ja eine adäquate Versorgung durch die Angehörigen gerade nicht gewährleistet war.

- $\quad$ im zweiten Fall nur dann den gleichen Effekt haben, wenn die altruistische Motivation, die ja - wie oben skizziert - im allgemeinen eine Bereitschaft zu finanziellen Opfern einschließt, gerade so bemessen ist, daß sie des finanziellen Verstärkers einer bei Heimpflege drohenden Kostenbeteiligung bedarf. ${ }^{131}$ Es müßte dann neben (2a) auch gelten, daß

(3) $X-L P<E-L E$.

Allerdings wird auch die Größe LP durch die Einführung der Pflegeversicherung tangiert, wenn deren Leistungskatalog auch ambulante Hilfen bei der häuslichen Pflege enthält. Die dadurch bewirkte Minderung der Pflegelast wirkt der durch den Wegfall von $\mathrm{V}$ eintretenden Verschiebung der Entscheidungsbedingungen entgegen. ${ }^{132}$ Wie sich die Ausmaße beider gegenläufigen Veränderungen zueinander verhalten, wird von Fall zu Fall variieren. Somit ist auch ein versicherungsinduziertes Umkippen der Entscheidung in beide Richtungen möglich:

- $\quad$ Familien, die bei geringer Pflegemotivation einen starken finanziellen Anreiz zur Übernahme der Pflege hatten, werden sich eventuell trotz verbesserter häuslicher Pflegebedingungen nunmehr für die stationäre Lösung entscheiden.

- Hat hingegen bei umgekehrter Motivationsstruktur die Schwere der Pflegelast den Ausschlag bei der Entscheidung für das Heim gegeben, so können die mit der Versicherung verbundenen Veränderungen der Parameter den Übergang zur häuslichen Pflege bewirken. Dies ist insbesondere auch dann zu erwarten, wenn die Entlastung durch ambulante Dienste die Übernahme einer Erwerbsarbeit durch die Pflegeperson ermöglicht.

\footnotetext{
${ }^{130}$ Dies impliziert, gleichsam als "Worst case"-Annahme, daß keine echte Selbstbeteiligung an den Heimkosten eingefordert wird.

${ }^{131}$ Erinnert sei an die im vorigen Abschnitt aufgeworfene Frage, ob der finanzielle Vorteil überhaupt als "Verstärker" wirkt.

${ }^{132}$ Diesen Zusammenhang betont auch G. Igl (1986), S. $199 \mathrm{f}$.
} 
Bis hierher ist gezeigt worden, daß eine als Moral hazard einzustufende Reaktion der Angehörigen in bezug auf die Entscheidung über den Einzug ins Pflegeheim nur für ganz spezielle Konstellationen zu erwarten ist. Gleichzeitig löst die Pflegeversicherung auch Effekte aus, die für sich betrachtet eine Entscheidung gegen die stationäre Pflege begünstigen. Das liegt daran, daß die Angehörigen auch ohne Versicherung nicht von der vollen Last der Heimpflegekosten betroffen sind. Der Saldo beider Wirkungen kann nicht ohne weiteres vorhergesagt werden. Je stärker eine Pflegeversicherung auf die Entlastung pflegender Angehöriger ausgerichtet wird, desto weniger berechtigt erscheint die Vermutung, daß ihre Einführung einen Anstieg der Heimquote auslösen wird.

Wenn sie dies dennoch tut, so sind dabei vermutlich auch Fälle enthalten, in denen die Entscheidung der Angehörigen für das Pflegeheim auch den Pflegebedürftigen selbst zugute kommt, da sie vorher nicht adäquat versorgt worden sind. Unter diesen Umständen liegt kein Moral hazard vor. Vielmehr handelt es sich hier um die Ausschaltung eines Fehlanreizes, der im versicherungslosen Zustand dann wirksam werden kann, wenn die Pflegebedürftigen in ihrer Entscheidungsfähigkeit eingeschränkt und die Angehörigen rücksichtslos sind.

Weiter verkleinern läßt sich das "Restrisiko" des Moral hazard schließlich dadurch, daß für die Größe V, also den bei Eintritt der Versicherung fortfallenden finanziellen Vorteil der häuslichen Pflege, innerhalb der Versicherung partiell Ersatz geschaffen wird. Dies könnte zum einen durch eine echte Selbstbeteiligung der Leistungsempfänger an den Heimkosten geschehen. Eine solche muß freilich sehr deutlich unter den Gesamtkosten liegen, um die Versicherung nicht ad absurdum zu führen. Ergänzend käme daher auch eine - wie immer bemessene - Entlohnung der privat Pflegenden in Betracht, sofern nicht befürchtet werden muß, daß sie deren altruistischen Antrieb untergräbt.

Die Gewährung von Geldleistungen an die Angehörigen birgt aber - wie oben besprochen - ihrerseits spezielle Anreize zum Moral hazard, indem es lohnend wird, Pflegebedürftigkeit zu simulieren oder ihren Schweregrad zu überzeichnen. Zudem würde auch in dieser Situation die für den versicherungsfreien Zustand beschriebene Gefahr der Vernachlässigung wehrloser Pflegebedürftiger bestehen und würde im Fall 
von Familien mit dringlichem Geldbedarf sogar verstärkt. ${ }^{133}$ Damit droht bei einer solchen Gestaltung eine partielle Effektivitätseinbuße; das Sicherungsziel würde nur eingeschränkt erreicht.

Wird eine solche Lösung dennoch gewählt, so erwächst aus dieser Gefahr ein weiteres Argument für die Bereitstellung professioneller Dienste zur Unterstützung der häuslichen Pflege: Zum einen gewährleistet die ambulante Betreuung durch professionelle Kräfte je nach Ausmaß selbst eine gewisse Mindestqualität, die vom Einsatz der Angehörigen unabhängig ist; zum andern kann sie eine Kontrollfunktion haben, indem eine eventuelle Vernachlässigung eher festgestellt und Abhilfe geschaffen werden kann. ${ }^{134}$

Abschließend sei klargestellt, daß die in diesem Abschnitt gewählte schematische Formulierung der Entscheidungssituation Angehöriger bestenfalls eine grobe und selektive Annäherung an reale Verhaltensweisen Betroffener sein kann. In Wirklichkeit ist die Entscheidung über die Pflegeform, sofern sie von den Angehörigen getroffen oder beeinflußt wird, nicht einfach ein Saldieren monetärer bzw. ohne weiteres auf der Nutzenebene mit Geldbeträgen kompatibler Größen. Soziologischen Untersuchungen $^{135}$ zufolge ist der Entscheidungsprozeß in Wirklichkeit ein sehr vielschichtiges psychosoziales Geschehen, bei dem gerade finanzielle Anreize die geringste Rolle zu spielen scheinen. ${ }^{136}$ Diese Ebene kann freilich hier nicht bearbeitet und soll daher auch nicht gegen die ökonomisch orientierten Ansätze ausgespielt werden. Dennoch ist es wichtig, sich ihrer realen Bedeutung bewußt zu sein.

\subsubsection{Zusammenfassung}

Zusammenfassend ist festzustellen: Der Begriff des Moral hazard bedarf in Anwendung auf die Pflegeversicherung einer sorgfältigen Abgrenzung, die anhand der Schutzziele

\footnotetext{
${ }^{133} \mathrm{vgl}$. DIW (1993), S. 422

${ }^{134}$ Diese Idee hat offenbar Eingang in die Gestaltung der deutschen Pflegeversicherung gefunden (Vgl. Kapitel 7).

${ }^{135}$ vgl. etwa H. Braun (1987), S. 24ff. bzw. die dort referierte Studie

${ }^{136}$ Auch dies hat vermutlich G. Igl (1986), S. 196f., im Auge, wenn er in anderem Zusammenhang feststellt: "Die Verhältnisse auf dem 'Markt' an sozialen Dienstleistungen liegen so einfach nicht, als daß man - behaftet mit simplen Vorstellungen von Nachfrage und Angebot - auf den Eintritt bestimmter Steuerungseffekte hoffen könnte."
} 
vorzunehmen ist: Nicht jede Verhaltensänderung, die durch die Einführung der Versicherung induziert wird und eine Verbrauchszunahme bei den entsprechenden Leistungen bewirkt, kann als unerwünschter Moral hazard im Sinne eines Effizienzproblems gewertet werden. Vielmehr ist gerade die Behebung von Versorgungsmängeln und -lücken, die mit dem Fehlen einer Versicherung zusammenhängen, neben dem Aspekt der finanziellen Absicherung ein zentrales Ziel der sozialpolitischen Maßnahme.

Verfehlt wäre es andererseits auch, die Anreizproblematik mit diesem Argument von vornherein niederzuschlagen. Es kann im Grundsatz durchaus nicht ausgeschlossen werden, daß die Versicherung unter bestimmten Umständen einen Leistungsverzehr anregt, der das im Sinne der Zielvorgabe Nötige nach Umfang und/oder Kosten übersteigt. Ein solches Phänomen, das sei hier ergänzend angemerkt, ist nicht allein effizienzschädlich, sondern bedroht im übrigen pragmatisch betrachtet indirekt auch die Zielwirkung der Pflegeversicherung: Schon weil die Beiträge als Zwangsabgaben auf der politischen Ebene stets indirekt mit den Steueransprüchen des Staates konkurrieren, unterliegt ihr Umfang einem ständigen Druck nach unten.

Sind aber insofern die Mittel in der Summe begrenzt, so geht ihre Fehlnutzung tendenziell zu Lasten von berechtigten Leistungsansprüchen. Und mehr noch: In dem $\mathrm{Maß}$, wie sich bei den Versicherten der Eindruck durchsetzt, daß die von ihnen aufzubringenden Beiträge einerseits verschwendet oder zweckentfremdet werden und/oder andererseits dort fehlen, wo sie wirklich benötigt werden, verliert sich die Vorteilhaftigkeit der Versicherung im Pareto-Sinn.

Immer mehr Beitragszahler werden aufhören, in der Existenz der Pflegesicherung per saldo eine Verbesserung ihrer Lage zu erblicken. In letzter Konsequenz müßte und würde dies zu deren Abschaffung führen. Auch darum war es erforderlich, differenziert zu prüfen, wo, wie, unter welchen Bedingungen und in welchem Ausmaß bei der Pflegeversicherung mit Moral hazard-Effekten zu rechnen ist. Der Befund aber ist überwiegend negativ:

Bei zweckmäßiger Gestaltung dürften von einer Pflegeversicherung keine nennenswerten Anreize zum Moral hazard für die (potentiell) Pflegebedürftigen selbst ausgehen. Plausibler begründbar erscheint zunächst die Vorstellung, daß eine Ausbeutung der Versicherung durch die Angehörigen erfolgt, die sich auf diese Weise ihrer "Pflicht" 
entziehen, die Pflege selbst in die Hand zu nehmen. $\mathrm{Zu}$ denken ist dabei vor allem an den Fall einer "unnötigen" Heimeinweisung. Offenkundig basiert aber diese Vorstellung auf der Prämisse, daß eine entsprechende Verpflichtung, Pflege im Rahmen des Möglichen selbst zu leisten, überhaupt besteht. Aus juristischer Sicht jedoch erweist sich eine solche Ausgangsannahme im typischen Fall der Pflege durch erwachsene Kinder als unhaltbar.

Ein von einer Pflegeversicherung ausgehender Moral hazard-Effekt kann sich also nur auf die konkreten finanziellen Folgen beziehen, die die Pflegebedürftigkeit eines Elternteils für die Kinder hat und von denen sie durch die Existenz einer Versicherung befreit würden. Diese Kostenbelastung im versicherungslosen Zustand ist aber in aller Regel nicht so groß, daß sie den Verzicht auf eine Heimeinweisung "lohnend" erscheinen ließe. Folglich darf im allgemeinen davon ausgegangen werden, daß bereits ohne Versicherung Familienpflege nur dann geleistet wird, wenn die Pflegenden sich für das Wohl des bedürftigen Angehörigen verantwortlich fühlen.

Ein solches nichtmonetäres Motiv wird oftmals auch dann ausschlaggebend bleiben, wenn mit Einführung einer Versicherung die mit der häuslichen Pflege verbundene Ersparnis entfällt. Zusätzlich wird die relative Attraktivität der Pflege im privaten Umfeld erhöht, wenn sie dank der Versicherung professionelle Unterstützung erfährt. Wer hingegen allein um des finanziellen Vorteils willen nur notdürftig gepflegt hat, wird beim Übergang in die versicherte Lage dazu bewogen, eine Heimeinweisung zu erwirken. Darin aber liegt kein Effizienzverlust, sondern ein erwünschter Beitrag zur Realisation des Schutzziels.

Ergänzend ist in diesem Zusammenhang darauf hinzuweisen, daß sich in modernen Industriegesellschaften in vielfältiger Weise objektive Verhältnisse und kollektive Orientierungen herausgebildet haben, die einer privaten Übernahme dauerhafter pflegerischer Aufgaben in vielen Fällen praktisch unüberwindlich im Wege stehen. Die sinkende Verbreitung und Stabilität ehelicher Bindung und die verminderte durchschnittliche Kinderzahl zählen ebenso dazu wie zunehmende Integration von Frauen in den Erwerbsprozeß und die steigende Mobilität in bezug auf den Wohnort. ${ }^{137}$ In diesen Fällen scheiden Angehörige als Träger einer informellen "Pflegeversicherung" und damit als potentielle Urheber von Moral hazard ohnehin aus.

${ }^{137}$ vgl. K.-D. Henke (1990), S. 4 
Die Vorstellung eines Normalfalles, in dem gerade auch bei fortgeschrittener Pflegebedürftigkeit die Familie regelmäßig als Alternative zum Pflegeheim zur Verfügung steht, ist überholt; eine an ihr orientierte Strategie zur Vermeidung vermeintlicher Anreizprobleme im Sinne des Moral hazard birgt die Gefahr inakzeptabler Sicherungslücken. ${ }^{138}$ Der Wirklichkeit heutiger Industriegesellschaften ist es angemessen, den Schutz vor den Folgen der Pflegebedürftigkeit als individuellen Anspruch an ein kollektives System zu gestalten ${ }^{139}$, anstatt Betroffene vorrangig an ihre Angehörigen zu verweisen. Eine solche Grundlinie schließt in keiner Weise aus, daß private Möglichkeiten und Bereitschaften zur Betreuung Pflegebedürftiger dort genutzt und gefördert werden, wo sie vorhanden sind. Die unterstützende Ausschöpfung solcher Potentiale stellt vielmehr ein entscheidendes Moment einer "guten" Versicherungslösung dar, und zwar gleichermaßen unter Zielaspekten wie auch in Hinblick auf die Effizienz.

Unberücksichtigt geblieben ist die "externe" Spielart des Moral hazard, soweit sie die Anbieter von Pflegeleistungen betrifft. Sie tritt dann auf, wenn die Versicherung so gestaltet ist, daß der Mechanismus der Preisbildung am Markt gestört wird. ${ }^{140}$ Bei einer pauschalen Geldleistung wird dies nicht oder am wenigsten der Fall sein, in einem Sachleistungssystem versagt die normale Marktpreisbildung vollends. Somit führt die Debatte zum einen zum Problem der Leistungsform zurück, das bereits unter dem Blickwinkel der Effektivität behandelt worden ist. Dort ist darauf hingewiesen worden, daß Pflegebedürftige am Markt oftmals nur eingeschränkt agieren können. Damit ist fraglich, ob die Marktpreisbildung im Fall der Pflege effizient funktioniert.

Als Alternative wird es im Fall eines Sachleistungssystems zwingend zur Bildung von Verhandlungspreisen kommen. Deren "eigentliche Unterlegenheit (...) liegt in dem

\footnotetext{
${ }^{138}$ Dies gilt etwa für das von R. Thiede (1990), S.178ff., entwickelte Konzept, in dem Beiträge und Geldleistungen der Pflegeversicherung sich danach richten, ob der Versicherte verheiratet ist und wieviele Kinder er hat. Dieses vorwiegend mit Überlegungen zur Anreizstruktur begründete Modell würde wohl in beträchtlichem und zunehmendem Umfang Sozialhilfefälle eintreten lassen, wenn nämlich Kinder zwar existieren und damit leistungsmindernd wirken, zur Übernahme der Pflege aber nicht bereit oder in der Lage sind.

139 "Alle Systeme (...) zielen gerade darauf ab, dem einzelnen im Alter unabhängig von der Versorgung durch eigene Kinder Sicherheit zu garantieren" (F. Hase (1992), S. 527). HASE, der diese Feststellung für die Altersvorsorge trifft, erblickt darin den "entscheidenden evolutionären Sprung (...), den die Alterssicherung mit dem Übergang zur modernen Gesellschaft vollzogen hat."

${ }^{140}$ siehe M. Nell (1992), S. $224 \mathrm{ff}$.
} 
vergleichsweise sehr hohen Zeitbedarf" ${ }^{141}$, wie NELL in bezug auf das sehr vielfältige und veränderliche Leistungsgefüge der Krankenversicherung veranschaulicht. Es ist anzunehmen, daß dieser Nachteil im übersichtlicheren "Pflegemarkt" weniger stark ins Gewicht fällt.

Somit relativiert sich die Überlegenheit der Preisbildung am Markt im vorliegenden Zusammenhang von beiden Seiten und es wird uneindeutig, ob die Gefahr eines externen Moral hazard überhaupt besteht. Ihre Vernachlässigung erscheint daher vertretbar.

Im übrigen ist pragmatisch anzumerken, daß diese Form des Moral hazard im Fall der Heimpflege stärker als die anderen Varianten auch schon ohne Versicherung auftreten kann. Übernimmt die Mindestsicherung wie in der Bundesrepublik regelmäßig die "Spitze" der Pflegekosten, so müssen die Pflegesätze ohnehin zwischen den Trägern der Heime und denen der Sozialhilfe ausgehandelt werden. So gesehen ist der externe Moral hazard unabhängig von seinem Gewicht kein Problem, das mit der Einführung einer Pflegeversicherung verbunden wäre.

Allerdings kann ein externer moral hazard nicht nur bei der Preisbildung, sondern auch in bezug auf die Mengen auftreten. Dieser Aspekt ist aus dem Gesundheitswesen wohlbekannt, wo der Arzt prinzipiell in der Lage ist, die Nachfrage nach seinen Leistungen selbst auszuweiten. Bei der Pflege ist dem aber relativ leicht vorzubeugen, indem die Feststellung des Pflegebedarfs und seines Ausmaßes nicht den Anbietern, sondern einer unabhängigen Instanz übertragen wird.

${ }^{141}$ M. Nell (1992), S. 160 


\section{Die gesetzliche Pflegeversicherung in den Niederlanden}

\subsection{Die Entstehungsgeschichte des AWBZ und seine ursprüngliche Form}

Die datierbare Geschichte der Pflegeversicherung in den Niederlanden beginnt am 24. August 1962. An diesem Tag wendet sich der Minister für Soziales und Volksgesundheit an den Sozialökonomischen Rat (SER) mit der Bitte um eine Stellungnahme zur Einführung einer Versicherung gegen "schwere gesundheitliche Risiken". Im Fehlen einer Regelung für diesen Bereich "sehr schwerer Lasten, die (...) jeden betreffen können und von niemandem ohne Hilfe des Staates oder von Dritten getragen werden können"1, erblickt er eine Lücke, die zur Abrundung des Krankenversicherungssystems zu schließen sei. Zwar ist der Pflegebereich, um den es dabei in erster Linie geht, vom Leistungskatalog der für Arbeitnehmer obligatorischen gesetzlichen Krankenversicherung nicht gänzlich ausgeklammert; die Erstattung entsprechender Kosten beschränkt sich jedoch auf 50\% und kann innerhalb eines Dreijahres-Zeitraums nur für ein Jahr in Anspruch genommen werden. ${ }^{2}$ Ferner ist auch die Deckung von Krankenhauskosten im Rahmen des herkömmlichen Krankenversicherungsgesetzes (ziektefondswet, ZFW) auf ein Jahr begrenzt.

Überdies umfaßt die GKV bei weitem nicht die gesamte Bevölkerung. Private Pflegeversicherungen werden nicht angeboten und "es erscheint bislang unmöglich, auf privatem Wege zu einer Lösung zu gelangen". ${ }^{3}$ Statt dessen schwebt dem Minister eine Sozialversicherung unter Einschluß der Gesamtbevölkerung vor, die auf einkommensproportionalen Prämien beruhen soll. Derartige Volksversicherungen existieren in den Niederlanden damals bereits für die Bereiche Rente und Hinterbliebene, während die staatlich geregelte Sicherung im Fall von Invalidität und Krankheit arbeitnehmerbezogen organisiert ist.

Sowohl der SER als auch die übrigen Beratungsgremien, die - wie im niederländischen Gesetzgebungsverfahren üblich - in der Folgezeit konsultiert werden, schließen sich der Initiative des Ministers im wesentlichen an. Auch in der übrigen politischen und

\footnotetext{
${ }^{1}$ G.M.J. Veldkamp et al. (1966), S. 1. Hinweis: Sämtliche Zitate aus Quellen in niederländischer Sprache sind vom Verfasser selbst ins Deutsche übertragen worden.

${ }^{2}$ vgl. ebenda, S. 36

${ }^{3}$ ebenda, S. 1
} 
gesellschaftlichen Landschaft scheint das Projekt durchweg positiv aufgenommen worden zu sein. Dies zeigt sich etwa in der parlamentarischen Debatte, auf die unten näher eingegangen wird.

In der Begründung des Gesetzentwurfs, der 1967 vorliegt, wird besonders dessen Bedeutung als Beitrag zur Verwirklichung von Chancengleichheit hervorgehoben, in der die Autoren angesichts wachsenden gesellschaftlichen Wohlstands den "kulturellen Auftrag dieser Zeit" ${ }^{4}$ erblicken. In den diesbezüglichen Ausführungen an gleicher Stelle lassen sich drei Aspekte trennen, unter denen die Pflegeversicherung dem gesellschaftlichen Oberziel dienen soll, nämlich

- $\quad$ erstens im Sinne einer einheitlichen Qualität und Verfügbarkeit verschiedenartiger gesundheitlicher Leistungen. Es wird konstatiert, daß die vom Gesetz betroffenen (pflegerischen) Bereiche - gemessen an der Entwicklung des Gesundheitswesens insgesamt - in Rückstand geraten seien. Diese These bezieht sich offenbar sowohl auf die Qualität als auch auf die Menge solcher Angebote.

- $\quad$ zweitens im Sinne des Anspruchs eines jeden auf größtmögliche Chancen zur Teilnahme am gesellschaftlichen Leben, der auf Basis einer verbesserten Versorgung eingelöst werden könne. Diese Vorstellung verweist bereits auf die große Bedeutung des Rehabilitationsziels bei der Bestimmung der Leistungen, die die Versicherung gewähren soll.

- drittens im Sinne des vorigen Punktes mit Blick auf die Angehörigen Pflegebedürftiger. Auch sie seien in ihren Entfaltungsmöglichkeiten durch die finanzielle und soziale Last der Pflege massiv beeinträchtigt.

In der Parlamentsdebatte über den Gesetzentwurf ${ }^{5}$ wird ein so motivierter Handlungsbedarf von keiner Seite des breiten Parteienspektrums in Abrede gestellt. Eher schon wird in Zweifel gezogen, daß die Volksversicherung auf Prämienbasis der richtige Weg zur Lösung des Problems sei. In der ministeriellen Begründung war hierzu angeführt worden, daß "der Solidaritätscharakter des Vorhabens so am deutlichsten (...) zum Ausdruck" käme. ${ }^{6}$ Ferner sei es wünschenswert, den Pflegebereich auf

${ }^{4}$ G.M.J. Veldkamp et al. (1966), S. 8

$s$ Tweede Kamer (1967)

${ }^{6}$ G.M.J. Veldkamp et al. (1966), S. 8 
diesem Wege an die Grundstruktur der bestehenden GKV anzuschließen, die sich "grosso modo" - bewährt habe.

Verschiedene Abgeordnete sprechen sich hingegen dafür aus, die Versorgung im Pflegefall als originäre Staatsaufgabe einzustufen und demgemäß aus Steuermitteln $\mathrm{zu}$ bestreiten. ${ }^{7}$ Diese Voten werden allerdings mit wenig Entschiedenheit vorgetragen und nur vage - etwa mit verwaltungspraktischen Argumenten - begründet. Vertreter kleinerer linker Parteien wenden sich aus Verteilungsgründen gegen die Beitragsfinanzierung. ${ }^{8}$

Auffällig ist die Bereitschaft der Sprecher, solche und andere Einwände angesichts der allseits empfundenen Dringlichkeit des Gesetzes zurückzustellen. Dies gilt auch für einige kritische Punkte des Entwurfs, die sich als bedeutsam für die weitere Entwicklung der Pflegeversicherung erweisen werden:

- $\quad$ Die Beschränkung des Leistungsbereichs auf stationäre ("intramurale") Einrichtungen wird sowohl im Parlament als auch vom Minister als problematisch erkannt. Dabei wird neben Gerechtigkeitserwägungen auch der hiervon ausgehende Fehlanreiz zugunsten der Heimunterbringung herausgestellt. ${ }^{9}$ Die Einbeziehung ambulanter Dienste wird von vornherein ins Auge gefaßt und unterbleibt einstweilen nur, um nicht das Gesetz in der Einführungsphase zu überfrachten bzw. sein Inkrafttreten zu verzögern. ${ }^{10}$ Der Gesetzestext ermöglicht die Ausweitung des Leistungskatalogs auf dem Verwaltungswege. ${ }^{11}$

- In Artikel 6.2 ist - als Kann-Bestimmung - die Erhebung eines Kostenbeitrags beim leistungsberechtigten Versicherten vorgesehen. Zur Begründung dieser Klausel dient der Hinweis, daß bei Unterbringung in einer Pflegeeinrichtung ein Teil der üblichen Lebenshaltungskosten eingespart und zudem in vielen

\footnotetext{
S. 271 bzw. 274. (S. 280).

9 vgl. Tweede Kamer (1967), S. 277 (Abg. de VREEZE)

${ }^{10} \mathrm{vgl}$. Veldkamp et al. (1966), S. 14

" siehe Tweede Kamer (1966), Artikel 6.3
}

${ }^{7}$ Vgl. die Redebeiträge der Abgeordneten RIETKERK und van MAENEN in Tweede Kamer (1967),

${ }^{8}$ Siehe ebenda die Stellungnahmen der Abgeordneten van der LEK (S. 282) und WOLFF 
Fällen vom Betroffenen auf dessen Erben übergehen würde. ${ }^{12}$ Für die im Gesetz offengelassene Gestaltung des Eigenbeitrags werden neben einem Fixum auch variable Zahlungen in Betracht gezogen, die entweder vom Gesamteinkommen des Betroffenen oder von den staatlichen Transfers abhängen könnten, die er bezieht. Im Parlament werden Zweifel daran laut, daß eine solche Regelung mit dem Sozialversicherungs-Gedanken vereinbar ist. ${ }^{13}$ Von dieser Frage wird sich zeigen, daß sie eine Konfliktlinie markiert, die noch heute von höchster Virulenz ist. Zunächst aber wird entschieden, den Selbstbehalt auf eine eher geringfügige Pauschale zu beschränken.

- $\quad$ Hinsichtlich des Rahmens, in dem die Kosten der geplanten Versicherung zu sehen sind, wird seitens des Ministers wie auch in der Parlamentsdebatte bereits damals die Auffassung vertreten, daß "die gesamte Prämienlast der Sozialversicherung jetzt ihre Grenze erreicht" habe. ${ }^{14}$ Auf dem Hintergrund dieser Einschätzung plant die Regierung, sich an der Finanzierung des neuen Versicherungszweiges mit einem Zuschuß aus Steuermitteln zu beteiligen, dessen Höhe sich nach den Kosten richten soll, die der Staat bei Fortbestand der versicherungslosen Lage vor allem in Form von Sozialhilfe zu tragen hätte. Da dies im ersten Jahr rund zwei Drittel der Gesamtkosten betrifft, wird ein Beitragssatz von nur rd. 0,5\% zur Finanzierung des letzten Drittels für 1968 ins Auge gefaßt. ${ }^{15}$ Gleichzeitig wird aber bereits angekündigt, daß der Staatsanteil später für das AWBZ entfallen und statt dessen im Kindergeldbereich gewährt werden soll, der seinerzeit ebenfalls Teil der Sozialversicherung war. ${ }^{16}$ Nehme man die Auswirkung dieser Operation vorweg, so sei zur vollständigen Finanzierung aus Prämien ein Satz "in der Größenordnung von $1,6 \%$ " erforderlich.

In der Tat ist der Zuschußanteil aus dem Staatshaushalt in den Folgejahren rasch abgebaut worden. ${ }^{17}$ (Schon von daher ist es übrigens hochgradig irreführend, zum

\footnotetext{
${ }^{12} \mathrm{vgl}$. Veldkamp et al. (1966), S. 26

${ }^{13}$ siehe Tweede Kamer (1967), S. 266 (Abg. van LEEUWEN)

${ }^{14}$ Veldkamp et al. (1966), S. 22

15 ebenda

${ }^{16}$ siehe auch Kikkert et al. (1966), S. 5

${ }^{17}$ vgl. L. Andriessen (1984), S. 383
} 
Nachweis einer "Beitragsexplosion" auf den anfänglichen Satz zu rekurrieren, wie dies in der deutschen Diskussion gelegentlich geschieht. ${ }^{18}$ )

Weiterhin ergibt sich aus dem ersten (und indirekt auch aus dem zweiten) der genannten Teilziele zur angestrebten "Chancengleichheit", daß ein (aufholender) Ausbau der Kapazitäten bei gleichzeitig verbessertem Leistungsniveau, der zu deutlichen Kostensteigerungen führen muß, ausdrücklich angestrebt worden ist. Bereits im Zuge des Gesetzgebungsprozesses hat das Sozialministerium eine diesbezügliche Schätzung des Mehrbedarfs vorgelegt, der sich aus dem damaligen Fehlbestand an Pflegebetten ergebe. ${ }^{19}$ Bei der Bewertung der tatsächlichen Kostenentwicklung gilt es dies zu berücksichtigen.

Das "Algemene Wet Bijzondere Ziektekosten" (AWBZ) wurde im Oktober 1967 mit großer Mehrheit verabschiedet und ist ab dem 1. Januar 1968 stufenweise in Kraft getreten.

\subsection{Eine kleine Chronik wesentlicher Veränderungen im System der Pflege- versicherung bis Anfang der neunziger Jahre}

Hier soll auf fünf Punkte eingegangen werden, an denen im Lauf der Jahre nach 1968 einschneidende Veränderungen im AWBZ bzw. den auf dieses Gesetz bezogenen Ausführungsbestimmungen eingetreten sind, die den hier untersuchten Bereich der Pflegeversicherung berühren. Vollständigkeit ist dabei nicht angestrebt; vielmehr werden in chronologischer Folge diejenigen Reformschritte herausgearbeitet, die im Sinne des Untersuchungsziels relevant erscheinen. Während die ersten vier Punkte vorwiegend die Leistungsseite betreffen, kommt im letzten die finanzierungsseitige Neuordnung der Volksversicherungen insgesamt zur Sprache. Die jüngsten Neuerungen

\footnotetext{
${ }^{18}$ Damit wird auch ignoriert, daß sich der Zuständigkeitsbereich der Versicherung auch außerhalb des Pflegewesens erheblich ausgeweitet hat. Besonders unredlich ist es, wenn der im Jahre 1968 letztlich gültige Satz von $0,4 \%$ verwendet wird, um ohne jede Erläuterung behaupten zu können, die Beitragslast sei inzwischen "um mehr als das Dreizehnfache gestiegen" (Frankfurter Institut(1991), S. 2; im gleichen Sinn auch H.C. Uleer (1991), S.54). Der genannte Satz erklärt sich nämlich auch daraus, daß die meisten Leistungen erst ab April 1968 gewährt wurden, während die Beitragspflicht schon mit Jahresbeginn in Kraft trat (siehe Tweede Kamer (1967), S. 261). Derlei Propaganda wird dem Publikum in der Bundesrepublik unter den Titeln "Argumente zur Wirtschaftspolitik" bzw. "Orientierungen" angeboten. ${ }^{19}$ siehe Kikkert et al. (1966), S. 5
} 
in Zusammenhang mit der Reform des niederländischen Gesundheitswesens werden erst am Schluß dieses Kapitels behandelt.

a) Die Aufnahme teilstationärer und ambulanter Dienste in den Leistungskatalog 1977 und 1980

Wie erwähnt wurde die Erweiterung des Leistungsspektrums der Volksversicherung über die Finanzierung von Pflegeheimplätzen hinaus bereits im Vorfeld der Einführung des AWBZ diskutiert und von den maßgeblichen Akteuren als sinnvoll betrachtet. Bis zur Realisierung entsprechender Pläne vergingen jedoch rd. 10 Jahre: Den ersten Schritt stellt dabei die Finanzierung der "Tagesbehandlung" in Pflegeheimen dar, die am 1. Juli 1977 aufgenommen wurde. ${ }^{20}$ Diese teilstationäre Betreuungsform ist schon angesichts des Transportaufwands - nur in einer begrenzten Zahl von Fällen sinnvoll und stellt daher bis heute nur ein recht kleines Segment im Spektrum der Pflegeangebote dar, dessen Bedeutung allerdings kontinuierlich zugenommen hat. 1989 entfielen rd. 4\% aller Pflegetage auf diesen Bereich ${ }^{21}, 1980$ lag sein Anteil erst halb so hoch.

Zum 1. Januar 1980 wurde auch die Finanzierung ambulanter Dienste auf das AWBZ übertragen. In erster Linie handelt es sich hier um das "Kruiswerk", eine landesweite, gemeinnützige Organisation in privater Trägerschaft, deren Arbeit zuvor überwiegend aus Mitteln der öffentlichen Hand bezahlt worden war. ${ }^{22}$ Ferner gehört seit 1989 mit der "Gezinshulp" auch ein vorwiegend hauswirtschaftliches Element zum Leistungsspektrum des AWBZ. ${ }^{23}$ Die Kosten beider ambulanter Zweige sind zusammen inzwischen halb so hoch wie die aller Pflegeheime. Von ihrer konzeptionellen Bedeutung im Sinne der angestrebten "Pflege nach Maß" wird noch die Rede sein.

\footnotetext{
${ }^{20}$ vgl. D. Poske (1984), S. 295

${ }^{21}$ W. Groenen/R. van der Wouden (1991), S. 111, Tab. 5

2 vgl. D. Poske (1984), S. 296

${ }^{23}$ vgl. Joachim Müller (1991), S. 24
} 
b) Die Einführung und teilweise Erhöhung des einkommensabhängigen Selbstbehalts 1983 und 1988

Zum 1.Januar 1983 wurde der Eigenbeitrag, der - nach mehrmaliger Anpassung bis dahin eine Höhe von 876 hfl pro Monat erreicht hatte ${ }^{24}$, mit dem Ziel reformiert, ein von der Regierung festgesetztes Einsparvolumen von 160 Mio hfl zu realisieren. ${ }^{25}$ Seitdem ist diese Selbstbeteiligung einkommensabhängig, was als Option bereits bei Einführung des AWBZ kontrovers diskutiert worden war (siehe oben). Der von der zuständigen Ministerin geplante Höchstbetrag von hfl $2700^{26}$ erwies sich als nicht durchsetzbar und wurde um die Hälfte reduziert. ${ }^{27}$ Es ist aufschlußreich, die hierüber geführte parlamentarische Debatte nachzuvollziehen:

Zentral ist dabei zum einen wiederum die Frage, ob eine derart massive Erhöhung des Selbstbehalts mit dem Versicherungsgedanken vereinbar ist oder ob damit "die Axt an die Wurzeln unserer sozialen Versicherungsstruktur gelegt wird". ${ }^{28}$ Die Befürworter der Erhöhung halten der letzteren Ansicht den oben erwähnten offenen Wortlaut des Gesetzes entgegen und argumentieren im übrigen, daß "die Notwendigkeit zur Minderung des Prämiendrucks so brennend" sei ${ }^{29}$, daß die in Art. 6 AWBZ vorgesehene Option des einkommensabhängigen Beitrags ausgeschöpft werden müsse. Außerdem sei das Leistungsfähigkeitsprinzip, wie es auf der Beitragsseite ohnehin angewandt wird, der Volksversicherung durchaus konform. ${ }^{30}$

Was zum anderen die inhaltliche Rechtfertigung des Selbstbehalts betrifft, so verschiebt sich der Akzent deutlich vom Motiv der oben erläuterten "Haushaltsersparnis" auf das Ziel, den Aufbau von Vermögen bei den Leistungsbeziehern generell zu unterbinden. Als untragbar wird solche Vermögensbildung vor allem dann angesehen, wenn das dazu verwandte Einkommen der Pflegebedürftigen in staatlichen bzw. sozialversicherungsrechtlichen Transfers besteht.

\footnotetext{
${ }^{24}$ L. Andriessen (1984), S. 373

25 vgl. Tweede Kamer (1983), Nr. 11, S. $5 f$.

${ }^{26}$ siehe Periodiek voor Sociale Verzekering (PS) (1982), S. 1047

${ }^{27}$ Tweede Kamer (1982), S. 401 und 445

${ }^{28}$ So die Abgeordnete Beckers-de Bruijn in Tweede Kamer (1982), S. 398

${ }^{29}$ ebenda, S. 403 (Abgeordnete Oomen-Ruijten)

${ }^{30}$ So der zuständige Minister de Graaf, ebenda, S. 412.
} 
Es liegt in der Konsequenz dieser Vorstellungen, denenzufolge niemand mehr Einkommen übrigbehalten soll, als er konsumtiv benötigt, daß zwischen verschiedenen Gruppen von Leistungsempfängern zu differenzieren sei. Maßstab einer solchen Kategorienbildung hätten die jeweiligen tatsächlichen Möglichkeiten der Betroffenen zur Teilnahme am öffentlichen Leben zu sein. ${ }^{31}$ Diese rigoros bedürftigkeitsbezogenen Überlegungen, die in der 83er-Regelung schließlich keinen Niederschlag finden, gipfeln im Vorschlag der rechtsliberalen VVD, selbst den Einkommensrest, der als "Taschengeld" apostrophiert wird, dann zu streichen, wenn Schwerstpflegebedürftigen aufgrund ihres Zustandes jede Möglichkeit abgesprochen werden könne, ihn zu verausgaben. ${ }^{32}$

1987/88 wiederholt sich der geschilderte Ablauf: Wie fünf Jahre zuvor steht ein vorgegebenes Sparziel (diesmal in Höhe von 50 Mio Gulden) am Anfang der Diskussion und wieder wird letztlich vergeblich der "Grundgedanke der Sozialversicherung" beschworen. ${ }^{33}$ Anders als 1982 wird dem Differenzierungskonzept diesmal aber insofern Rechnung getragen, als die Erhöhung des Maximalbeitrags auf hfl 2.200 nur für Patienten über 65 Jahre gilt. ${ }^{34}$ Die Einwände des Ziekenfondsraad, der darin eine ungerechtfertigte Diskriminierung erblick ${ }^{35}$, sind unbeachtet geblieben.

Gescheitert ist hingegen zu Beginn der 90er Jahre der Versuch der Regierung, die Selbstbeteiligung auch nach dem Vermögen zu bemessen. ${ }^{36}$ Eine derart grundlegende Änderung erwies sich im Parlament als nicht mehrheitsfähig. ${ }^{37}$

c) Die Budgetierung der Pflegeheime seit 1984

Bis 1983 war die Finanzierung der Kosten stationärer Pflege gekennzeichnet durch ein Erstattungsverfahren. Die Kosten konnten sich ohne systematische Kontrolle entwickeln, da sie den Einrichtungen im Nachhinein aus Beitragsmitteln vergütet

\footnotetext{
${ }^{31}$ Siehe den Antrag der Abgeordneten Oomen-Ruijten und Terpstra, ebenda, S. 403.

${ }^{32}$ siehe Tweede Kamer (1983), Nr. 13, S.4

${ }^{33}$ siehe etwa Ziekenfondsraad (1987), S. 2

${ }^{34}$ Staatscourant vom 29. Juni 1988

${ }^{35}$ Ziekenfondsraad (1987), S. 6

${ }^{36}$ siehe de Volkskrant vom 17. Juli 1992: Ook thuis verpleegde moet vermogen gaan aanspreken

${ }^{37}$ siehe J. Bruinsma (1993)
} 
wurden..$^{38} \mathrm{Da}$ die Versicherer nur als Durchgangsstation im Finanzierungsstrom zwischen der Zentralkasse und den Pflegeheimen fungierten, bestand ihrerseits kein starkes $^{39}$ Interesse, Druck auf die Pflegesätze auszuüben. Mag eine solche Open-endRegelung in der Phase des politisch gewollten raschen Aufbaus zeitgemäßer Pflegeangebote noch angemessen erschienen sein, so erstaunt es nicht, daß sie danach in Hinblick auf das öffentliche Interesse an Effizienz und Kostenbegrenzung als untauglich angesehen wurde.

Seit 1984 wird den Pflegeheimen ebenso wie den Krankenhäusern zentral ein Gesamtbudget zugewiesen, das auf den ebenfalls staatlich kontrollierten Platzzahlen einerseits und andererseits auf normierten Kostensätzen pro Pflegetag basiert. Im zweiten Schritt wird diese Globalvolumen durch ein zentralstaatliches Gremium auf die Ebene der einzelnen Einrichtungen heruntergebrochen. ${ }^{40}$ Individuellen Besonderheiten der Heime wird dabei durch eine Differenzierung der Tagessätze Rechnung getragen. ${ }^{41}$

Ein Anreiz zur Unterschreitung des Heimbudgets ist insofern gegeben, als eventuelle Restmittel den Reserven der Pflegeeinrichtung zugeführt werden dürfen..$^{42}$ Auf MakroEbene wird das vorab aufgestellte Budget allerdings regelmäßig überschritten ${ }^{43}$, was in erster Linie auf überplanmäßige tarifliche Lohnsteigerungen zurückzuführen ist. ${ }^{44}$ $\mathrm{Da}$ aber dieser Faktor nicht dem Einfluß der einzelnen Heimleitung unterliegt und ein kompensierender Abbau von Personal offenbar nicht erwünscht ist, wird das Budget ggfs. im Nachhinein um den Einfluß der Lohnentwicklung korrigiert. Ebenso wird in bezug auf die allgemeine Preisentwicklung verfahren. ${ }^{45}$ Da die Lohnkosten der

\footnotetext{
${ }^{38}$ vgl. W. Groenen/R. van der Wouden (1991), S. 118

${ }^{39}$ Eine gewisse implizite Kontrolle folgt freilich daraus, daß bei erkennbar überhöhten Preisen mit politischen Reaktionen gerechnet werden muß. Es empfiehlt sich für die Beteiligten nicht, eine Regelung ohne systematische Kostenkontrolle überzustrapazieren.

${ }^{40}$ siehe Ziekenfondsraad (1993), S. 42

${ }^{41}$ Zur damit und durch nachträgliche Korrekturen (siehe unten) bewirkten Streuung der effektiven Pflegesätze siehe etwa Nationaal Ziekenhuis Instituut (NZI) (1989), S. 12f.

${ }^{42}$ Groenen/van der Wouden (1991), S. 118

${ }^{43}$ vgl. Ziekenfondsraad (1993), S. 44

${ }^{44}$ Nach B.B. Bakker/C. A. de Kam (1992), S. 213f. wird die Lohnentwicklung bei der Planaufstellung "konsequent viel zu gering geschätzt, vermutlich weil die Betroffenen die Zahlen (...) als Untergrenze (des tatsächlich Durchsetzbaren, S.W.) ansehen."
}

${ }^{45}$ Ziekenfondsraad (1993), S. 42 
mit Abstand größte Kostenfaktor in der stationären Pflege sind, ist die Wirkung der Budgetierung auf den Gesamtkostenverlauf gering geblieben; in bezug auf die sonstigen Kosten allein ist sie gleichwohl deutlich erkennbar. ${ }^{46}$

d) Die Einrichtung von Indikationskommissionen 1989

Die nun zu behandelnde Änderung ist in ihren Folgen wohl weniger gewichtig als die vorgenannten, wirft aber Licht auf einige problematische Aspekte des Leistungsanspruchs. Vor 1989 lag die Entscheidung über das Vorliegen von Pflegebedürftigkeit im Sinne stationären Bedarfs beim Hausarzt und mußte vom zuständigen "Ausführungsorgan" des AWBZ (also der jeweiligen Krankenversicherung) lediglich bestätigt werden. Eine solche Indikation führte aber nicht automatisch zur Aufnahme in ein Heim; die Entscheidung darüber lag und liegt auch heute bei der Pflegeeinrichtung selbst.

In den 80er Jahren ist dieses Verfahren dadurch unbefriedigend geworden, daß das Angebot an Pflegeplätzen im Wege der staatlichen Genehmigungspraxis beim Bau neuer Kapazitäten konsequent knapp gehalten wurde. Die Folge sind Wartelisten zunehmender Länge, die von den einzelnen Pflegeheimen nach eigenem Ermessen gehandhabt werden. ${ }^{47}$ Die auf diesen Listen geführten Menschen haben von Rechts wegen kraft ihrer Indikation einen Anspruch auf Unterbringung und es ist erstaunlich, daß gerichtliche Klagen auf dessen Einlösung bislang nicht bekannt geworden sind..$^{48}$ Die neue Regelung ${ }^{49}$ verlagert die Indikationsstellung auf eine spezielle lokale Kommission, die mit Zuständigkeit für die Vergabe von Altenheimplätzen bereits bestand und zumindest einen Arzt und einen Sozialarbeiter umfaßt.

Das oben skizzierte Problem wird damit zwar nicht gelöst, aber doch insofern gemildert, als das Gremium mit den umliegenden Heimen zusammenarbeitet oder Kontakt hält und dabei über die Dringlichkeit der Pflegefälle urteilt. ${ }^{50}$ Sofern die

\footnotetext{
${ }^{46}$ siehe Groenen/van der Wouden (1991), S. 116 bzw. 112f: Zwischen 1984 und 1989 sind die Nichtlohnkosten pro Pflegeplatz real um rd. $9 \%$ gesunken. - Nach ersten Meldungen zeitigt die Budgetierung der deutschen Kliniken derzeit ähnliche Effekte.

${ }^{47}$ siehe hierzu W.P. Elberse (1989)

${ }^{48}$ Groenen/van der Wouden (1991), S. 119

${ }^{49}$ Für eine ausführliche Darstellung siehe Vereniging van Nederlandse Gemeenten (1988).

so vgl. ebenda, S. 15
} 
Heime ihre Aufnahmeentscheidungen nach dieser Einstufung richten, ergibt sich immerhin ein kohärentes Vergabesystem, wo zuvor eine intransparente Vielfalt unterschiedlicher Koordinationsniveaus und Kriterien geherrscht hat. ${ }^{51}$ Zusätzlich hat die Kommission die Aufgabe, ambulante Betreuungsmöglichkeiten aufzuzeigen und zu vermitteln, wenn zwar Hilfsbedürftigkeit konstatiert, eine Heimeinweisung aber nicht für zwingend befunden wird.

Insofern dient das Indikationsverfahren einer genaueren Abstimmung von Angebot und Bedarf an Pflege im Einzelfall: zum einen sollen die Ansprüche der Versicherten zur Geltung gebracht, zum anderen eine Fehlallokation knapper Pflegebetten vermieden werden.

e) Die Neuordnung der Prämienerhebung im Zuge der "Oort-Operation" 1990

Zum 1. Januar 1990 ist in den Niederlanden ein neues System der Einkommensbesteuerung in Kraft getreten, das u.a. durch die Integration der Volksversicherungsbeiträge in den Steuertarif gekennzeichnet ist. ${ }^{52}$ Der neue Tarif kennt drei Stufen, wobei sich die Belastung in der untersten aus den Volksversicherungsprämien und einem Steueranteil zusammensetzt. Der Höchstbetrag dieser Stufe ist gleichzeitig die Beitragsbemessungsgrenze, so daß das Aufkommen aus den beiden höheren Tarifstufen zur Gänze dem Fiskus zufließt.

Gegenüber der zuvor praktizierten separaten und streng proportionalen Beitragserhebung ändert sich das den Beiträgen zurechenbare Belastungsmuster recht einschneidend, da nunmehr auch für diese ein Freibetrag gilt. Dessen indirekter Progressivwirkung steht gegenüber, daß die Beitragsbemessungsgrenze deutlich gesunken ist und somit der Bereich regressiver Belastung früher beginnt. An dieser Stelle genügt die Feststellung, daß die für Sozialversicherungen üblicherweise charakteristische Proportionalität der Lasten in bezug auf das Einkommen für die niederländische Pflegeversicherung nurmehr sehr eingeschränkt gegeben ist.

Ein weiterer bedeutender Aspekt der Oort-Operation ist die mit ihr verbundene Verlagerung der Beitragszahllast vom Arbeitgeber auf die Lohnempfänger. Der Effekt

\footnotetext{
${ }^{51}$ siehe etwa Elberse (1989), S. 41

${ }^{52}$ Näheres hierzu bei M.C.F. Meijer et al. (1989) und in Periodiek voor Sociale Verzekering (1990).
} 
dieser Änderung ist durch einen im Detail sehr komplizierten "Umschichtungszuschlag" auf Löhne und Gehälter im Ansatz voll kompensiert worden. In der Folgezeit ergeben sich bei steigenden Prämien und gleichzeitiger Nichtanpassung oder Aufhebung des Zuschlags jedoch sehr wohl Wirkungen auf die Primärinzidenz. ${ }^{53}$

Auf dem Hintergrund der hier skizzierten Entwicklungsprozesse im und um das AWBZ kann nunmehr die heutige Gestalt der Pflegeversicherung im Überblick dargestellt werden.

\subsection{Die derzeitige Gesetzeslage im einzelnen}

Im folgenden wird dargestellt, wie die Absicherung des Pflegerisikos in den Niederlanden derzeit geregelt ist. Die Ausführungen stützen sich teilweise auf die jeweils aktuellsten Angaben, die mir bis Herbst 1993 zugänglich waren. Dabei ist zu beachten, daß die Gültigkeitsdauer der verwandten Daten im Fall von Einkommensgrenzen, Freibeträgen, Prämiensätzen etc. dadurch begrenzt ist, daß solche Werte jährlich bzw. halbjährlich angepaßt werden. Quantitativ gravierende oder das System betreffende Veränderungen sind hingegen ggfs. bis zum endgültigen Redaktionsschluß Ende 1994 berücksichtigt worden.

Einige Regelungsaspekte sind bereits in den vorangegangenen Abschnitten zur Sprache gekommen und werden hier nurmehr näher ausgeführt. Elemente, die in direktem Zusammenhang mit der Reform des niederländischen Krankenversicherungswesens stehen, werden in Abschnitt 5.5 gesondert behandelt.

a) Die gesetzliche Basis

Grundlage der Pflegeversicherung ist das Algemene Wet Bijzondere Ziektekosten (AWBZ). ${ }^{54}$ Unter den auf Basis dieses Gesetzes gewährten Versicherungsschutz fällt die gesamte niederländische Wohnbevölkerung sowie im Ausland ansässige Personen,

\footnotetext{
53 vgl. Commissie voor de Belastingsherziening (1991), S. 15

${ }^{54}$ Die zugrundeliegende Textfassung ist abgedruckt im Staatsblad van het Koninkrijk der Nederlanden (1990).
} 
die der niederländischen Lohnsteuerhoheit unterliegen. ${ }^{55}$ Der Leistungsbereich des AWBZ umfaßt stationäre, teilstationäre und ambulante Hilfen in Sachleistungsform und ist breit gefächert. Einige wichtige Elemente außerhalb des hier zu untersuchenden Sektors der Altenpflege sind: ${ }^{56}$

- im stationären ("intramuralen") Bereich:

- Einrichtungen für geistig behinderte Kinder und Jugendliche

- Einrichtungen für Seh- und Hörbehinderte

- Psychiatrische Krankenhäuser,

- im teilstationären ("semimuralen") Bereich:

- Tagesheime für behinderte Kinder

- Altentagesstätten

- Einrichtungen für "geschütztes Wohnen" Behinderter

- im ambulanten ("extramuralen") Bereich:

- Fürsorgeeinrichtungen für geistig Behinderte

- Nicht-klinische Psychiatrie

- Impfprogramme

- Nachgeburtliche Betreuung

Zum 1.1.92 hinzugekommen sind die gesamte Arzneimittelversorgung, die Vorsorgeuntersuchungen bei Schwangerschaft sowie einige medizinische Hilfsmittel. ${ }^{57}$ Der Altenpflegesektor als Untersuchungsbereich dieser Arbeit setzt sich zusammen aus

- den Pflegeheimen,

- der Tagespflege (semimural) sowie

- den ambulanten Diensten des Kruiswerk (häusliche Pflege) und der "Familienhilfe" (Unterstützung bei der Haushaltsführung).

\footnotetext{
${ }^{5 s}$ Art. 5 in Verbindung mit Art. 2

${ }^{56}$ Für die komplette Auflistung siehe Stichting Centraal Administratie Kantoor AWBZ (1993), S. 52.

${ }^{57}$ siehe Ziekenfondsraad (1992), S. 11
} 
b) Leistungsanspruch und Kapazitätskontrolle

Das Gesetz gewährt den Versicherten "Anspruch auf Leistungen zur Befriedigung ihrer Bedürfnisse nach medizinischer Behandlung, Verpflegung und Versorgung", die "zum Erhalt, zur Wiederherstellung oder zur Förderung der Arbeitsfähigkeit oder (..) zur Verbesserung der Lebensumstände" dienen. ${ }^{58}$ Art und Umfang der Leistungen sind im Einzelnen außerhalb des Gesetzes zu regeln; ein Kostenbeitrag des Versicherten kann zur Voraussetzung des Leistungsempfangs gemacht werden und "braucht nicht für alle Versicherten gleich zu sein". ${ }^{59}$ Die aufgrund des letztgenannten Vorbehalts getroffenen Regelungen werden weiter unten behandelt. Zunächst ist zu zeigen, wie sich der allgemeine Leistungsanspruch im Bereich der Altenpflege konkretisiert.

In bezug auf die stationäre Pflege ist dafür zunächst die im entsprechenden Ausführungsbeschluß enthaltene Bestimmung entscheidend, wonach eine Indikation zur Aufnahme in ein Pflegeheim vorliegt, "wenn und solange für den Versicherten die Verpflegung und medizinische Behandlung in einer Pflegeeinrichtung vernünftigerweise angezeigt sind" ${ }^{60}$ Diese auf den ersten Blick nicht gerade gehaltvolle Formulierung ist in der Rechtsprechung seit Anfang der 80er Jahre dahingehend ausgelegt worden, daß nicht allein medizinische, sondern auch soziale Umstände das Vorliegen einer Indikation begründen können bzw. bei der Entscheidung darüber berücksichtigt werden müssen. ${ }^{61}$

Das neuerdings zur Feststellung der Indikation vorgesehene Verfahren ist bereits im vorigen Teilkapitel erläutert worden. Hier sei nochmals betont, daß nicht in allen Indikationsfällen eine sofortige Aufnahme in ein Pflegeheim erfolgt. Formal obliegt die Platzvergabe letztlich der Einrichtung selbst; faktisch ist festzustellen, daß die Gesamtkapazität der Heime hinter der Anzahl indizierter Patienten zurückgeblieben ist. Kontrolliert wird das landesweite Angebot an Pflegeplätzen vom Staat, da die in der Regel von Stiftungen errichteten und betriebenen - Heime auf eine staatliche Lizenz angewiesen sind. ${ }^{62}$ Auch der Ausbau einer schon anerkannten Einrichtung

\footnotetext{
${ }^{58}$ Artikel 6.1

${ }^{59}$ Artikel 6.2

${ }^{60}$ Kluwer Sociale Verzekeringswetten (1989), S. 24

${ }^{61}$ ebenda, S. 40

${ }^{62}$ vgl. P.J.H. Laurs/G.B. Teuben (1990), S. 1797
}

130 
bedarf der Genehmigung durch das Gesundheitsministerium. Die Lizenzvergabe ist im Wet Ziekenhuisvoorzieningen (WZV) geregelt und richtet sich nach gewissen Kapazitätsnormen: pro 1000 Einwohner über 64 Jahren wird eine bestimmte Anzahl von Plätzen als hinreichend angenommen. ${ }^{63}$ Das zwischen dem individuellen Pflegeanspruch und der globalen Kapazitätspolitik bestehende Spannungsverhältnis markiert einen neuralgischen Punkt des Pflegesystems. Es ist der Ort gerade in letzter Zeit intensivierter Versuche, das Versorgungsziel in Einklang mit dem Wunsch nach Kostenbegrenzung zu bringen. ${ }^{64}$

Neben der Heimpflege gehört zum Leistungskatalog des AWBZ auch ein dichtes Netz inhaltlich differenzierter häuslicher Betreuungsangebote, die vorwiegend von älteren Versicherten in Anspruch genommen werden. ${ }^{65}$ Der Umfang ambulanter Pflege ist pro Einzelfall in der Regel auf drei Besuche täglich bei einer Gesamtdauer von höchstens 2,5 Stunden beschränkt ${ }^{66}$, dabei ist ständige Rufbereitschaft gewährleistet. ${ }^{67}$ Zusätzlich kann Hilfe im Haushalt gewährt werden.

Bei der Indikation für die Inanspruchnahme häuslicher Dienste ist zu trennen zwischen den vorwiegend hauswirtschaftlichen Leistungen der "Familienhilfe" (Gezinshulp) und den Pflegeangeboten der "Kruiswerk"-Vereine. In bezug auf letztere besteht ein formalisiertes Verfahren bislang nicht; die jeweils zuständige Bezirkspflegekraft entscheidet kraft ihrer fachlichen Kompetenz selbst über die Hilfsanträge. ${ }^{68}$ Im Bereich der Haushaltshilfe erfolgt hingegen eine explizite Bedarfsbeurteilung durch darauf spezialisierte Mitarbeiter. Dem Verfahren liegt ein Subsidiaritätsgedanke insofern zugrunde, als nicht die Fähigḳeiten des bedürftigen Individuums allein ausschlaggebend sind, sondern die in dessen Haushalt insgesamt verfügbaren Selbsthilfe-Ressourcen. ${ }^{69}$ Zudem wird versucht, das jeweilige Hilfspaket nach Umfang und Inhalt möglichst exakt auf den tatsächlichen Bedarf zuzuschneiden.

\footnotetext{
${ }^{63}$ Näheres bei Kluwer Zorg en Dienstverlening (1990), S. 20.

${ }^{64}$ siehe etwa ebenda, S. 10

6s vgl. Landelijke Vereniging voor Thuiszorg (LVT), (1991), S. 29

${ }^{66}$ Nationale Kruisvereniging (1989), S. 39

${ }^{67}$ LVT (1990), S. 6

${ }^{68}$ vgl. LVT (1990a), S.5

69 ebenda
} 
Im Juni 1990 sind die bislang unabhängigen Einrichtungen des Kruiswerk und der Gezinshulp auf der Ebene ihrer Dachverbände eine Fusion eingegangen. ${ }^{70}$ Infolge des Zusammenschlusses wird derzeit ein einheitliches Indikationsverfahren erarbeitet, das in seinen Grundzügen dem oben für die hauswirtschaftlichen Hilfen skizzierten entsprechen wird. ${ }^{71}$ Analog zum stationären Sektor zeigt sich auch in der ambulanten Pflege eine wachsende Lücke zwischen dem durch die Indikationspraxis bestimmten Sollvolumen und dem im jeweils vorgegebenen finanziellen Rahmen tatsächlich realisierbaren Leistungsumfang. ${ }^{72}$ Wiederum gerät also das Indikationsverfahren in das Dilemma, entweder seine Kriterien nach der Haushaltslage richten zu müssen oder irrelevant zu werden, indem es Bedarf bescheinigt, der nicht gedeckt werden kann. Eine "Lösung" wird offenbar darin gesehen, daß man zwar an dem Grundsatz festhält, die Bedarfsermittlung ohne Rücksicht auf Mittelknappheit vorzunehmen ${ }^{73}$, zusätzlich aber ein Dringlichkeitskriterium anwendet, um "Willkür und Fahrlässigkeit" bei der Verteilung der Mittel auf eine zu große Zahl anerkannt Bedürftiger zu vermeiden. ${ }^{74}$.

c) Der Eigenbeitrag 75

Der Eigenanteil der Betroffenen an den Pflegekosten nimmt im Pflegeversicherungssystem eine Sonderstellung ein, da er zum einen als Leistungsvoraussetzung fungiert, zum anderen einen nicht unwesentlichen Beitrag zur Finanzierung des Pflegewesens darstellt. Außerdem sind die hierzu aufgestellten Regelungen so komplex, daß sie hier in einem eigenen Abschnitt behandelt werden sollen.

Wie oben bereits erwähnt, ist der Eigenbeitrag im Fall stationärer Pflege abhängig vom Einkommen des Heimbewohners. Dies allerdings nur dann, wenn nicht ein Partner existiert, der den zuvor gemeinsamen Haushalt weiterführt. Im Zuge des Strebens nach Gleichberechtigung nichtkonventioneller Lebensformen ist die Voraussetzung entfallen, daß es sich dabei um den Ehepartner handeln muß. Wie Verheiratete werden

\footnotetext{
${ }^{20}$ siehe LVT (1991), S. 5

${ }^{71}$ vgl. Stichting Centrale Raad voor Gezinsverzorging/Nationale Kruisvereniging (1990), S. $15 f$

${ }^{2}$ Für Näheres hierzu siehe Kapitel 6.1.3

${ }^{73}$ vgl. Stichting Centrale Raad voor Gezinsverzorging/Nationale Kruisvereniging (1990), S. 15

${ }^{74}$ LVT (1990a), S. $18 \mathrm{f}$.

${ }^{75}$ Diese Bezeichnung lehnt sich an den niederländischen Originalbegriff "eigen bijdrage" an. Sie wird hier synonym zur "Selbstbeteiligung" benutzt.
} 
zwei Personen beliebigen Geschlechts behandelt, die einen gemeinsamen Haushalt führen, ohne miteinander verwandt zu sein.

In diesem Fall wird nur ein pauschaler Mindestbetrag von $180 \mathrm{hfl}$ im Monat erhoben. Diese Regelung hängt offensichtlich mit dem ursprünglichen Verständnis des Eigenbeitrags als Gegenwert der häuslichen Ersparnis zusammen. Diese reduziert sich bei Fortbestand des Haushalts in den Augen des Gesetzgebers offenbar auf den obigen Festbetrag, der als Ernährungskostensatz interpretiert wird. ${ }^{76}$ Überzeugend ist dieser Begründungszusammenhang freilich schon deswegen nicht, weil der strikte Bezug auf die Haushaltsersparnis mit der Einführung des variablen Eigenbeitrags ausdrücklich aufgegeben worden ist. ${ }^{77}$ Auch ist die Beschränkung auf eingesparte Nahrungskosten im Fall der Zusammenwohnenden nicht einsichtig. In Anerkennung dieser Unstimmigkeit hat der Raad van Beroep in Haarlem einer Klage von seiten Alleinstehender stattgegeben und die Regelung als unvereinbar mit dem Diskriminierungsverbot eingestuft. ${ }^{78}$ Eine Umsetzung dieses Urteils steht aus.

Für Alleinstehende sowie für - im oben erläuterten Sinn -"Verheiratete", die beide in einer Pflegeeinrichtung leben, errechnet sich der Selbstbehalt wie folgt:

- vom gesamten Nettoeinkommen wird ein fester Freibetrag in Höhe von 4.111 hfl pro Jahr abgezogen (Verheiratete: $6.857 \mathrm{hfl}$, Stand 1. Juli 1992).

- $\quad$ Übersteigt das beitragsfähige Nettoeinkommen den Betrag von $10.308 \mathrm{hfl}$ jährlich, was immer dann der Fall sein wird, wenn der Betreffende über die staatliche Mindestrente hinaus sonstige Einkünfte bezieht, so dürfen von diesem Überhang bis zu 840 hfl zusätzlich in Abzug gebracht werden. ${ }^{79}$ Vom Einkommen oberhalb dieser Grenze bleiben nochmals 12,5\% außer Betracht.

- Gesondert abgezogen werden $15 \%$ eines evtl. vorhandenen Arbeitseinkommens sowie auch gewisse Lasten, die mit einer in Aussicht genommenen"Rückkehr in die Gesellschaft" verbunden sind (etwa die Mietkosten bei Behalt der

\footnotetext{
${ }^{76}$ vgl. Informatiecentrum Zorg en Welzijn (IZW) (1992), S. 7

$n$ vgl. Tweede Kamer (1982), S. 403; siehe auch oben, Abschnitt 5.2

${ }^{78}$ IZW (1992), S. 7f.

${ }^{79} \mathrm{Zu}$ den - technischen - Gründen dieser Klausel siehe unten.
} 
früheren Wohnung). Letztere Bestimmung greift nur dann, wenn eine Entlassung aus dem Pflegeheim für wahrscheinlich gehalten wird. ${ }^{80}$

- Das nach diesen Kürzungen verbleibende Einkommen wird als Eigenbeitrag eingezogen, sofern es einen monatlichen Betrag von $2.200 \mathrm{hfl}$ nicht übersteigt. Für Heimbewohner unter 65 Jahren beträgt dieses Maximum $1.350 \mathrm{hfl}$. Paare von Heiminsassen brauchen diese Höchstbeiträge nur einmal zu zahlen.

Grundsätzlich tritt die Pflicht zur Entrichtung des einkommensabhängigen Beitrags sechs Monate nach der Aufnahme in ein Pflegeheim in Kraft; bis dahin ist nur der Pauschalbetrag von monatlich $180 \mathrm{hfl}$ fällig. Vom ersten Tag der stationären Behandlung an voll beitragspflichtig sind allerdings Patientengruppen, bei denen nicht mit einer Rückkehr aus dem Heim zu rechnen ist. Insbesondere wird dies bei Vorliegen eines psychogeriatrischen Krankheitsbildes unterstellt ${ }^{81}$.

Da die Ermittlung des Eigenbeitrages auf Basis des Einkommens im Vorjahr erfolgt, sieht eine zusätzliche Klausel vor, daß ein minimaler Einkommensrest etwa in Höhe des o.g. Basisabzugs auch im Bezugsjahr selbst unangetastet bleibt. Ggfs. wird der Selbstbehalt entsprechend gekürzt. ${ }^{82}$

Die Abbildungen 5.1 und 5.2 enthalten einige graphische Darstellungen zum Belastungsverlauf, der sich aus der beschriebenen Regelung ergibt. Das etwas bizarre Bild, das sich hinsichtlich der Grenz- und Durchschnittslast in Abbildung 5.2 zeigt, ist in erster Linie auf die schmale Zone oberhalb der Standardrente zurückzuführen, in der der Eigenbeitrag absolut konstant bleibt. Begründet wird die Existenz dieses Bereichs mit der dadurch erzielbaren Verwaltungsvereinfachung: Der für die Bezieher der staatlichen Einheitsrente gültige Standardbeitrag soll auch bei Vorliegen geringfügiger Nebeneinkünfte Anwendung finden können. ${ }^{83}$

\footnotetext{
${ }^{80}$ ebenda, S. 20

${ }^{81}$ ebenda, S. $9 f$.

82 ebenda, S. 13

${ }^{83}$ vgl. ebenda, S. 18
} 


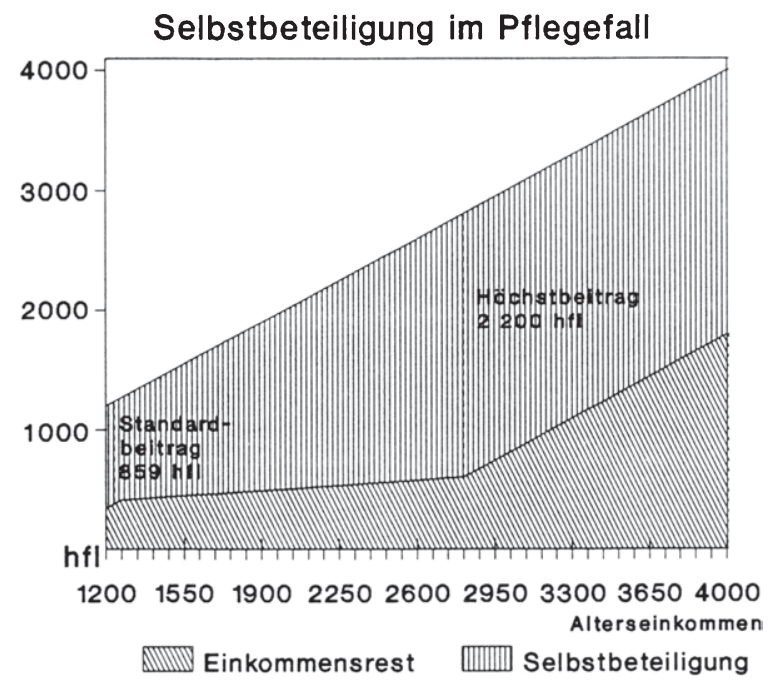

eigene Darstellung

Ansonsten ist die Beitragbemessung offenbar an der Vorstellung orientiert, den Einkommensrest auf das Nötige zu begrenzen, ohne jedoch eine völlige Nivellierung herbeizuführen. Die Regressivität der Belastung im Bereich hoher Einkommen steht zwar im Widerspruch zu diesem Konzept, kann aber nicht analog zu einem entsprechenden Steuertarif interpretiert werden. Vielmehr markiert der Höchstsatz die Grenze dessen, was als vereinbar mit dem Versicherungsgedanken sowie mit dem ursprünglichen Motiv der Haushaltsersparnis angesehen wird.

Im Durchschnitt liegt der von den Bewohnern aufgebrachte Kostenbeitrag im Jahre 1992 bei $848 \mathrm{hfl}$ im Monat ${ }^{84}$; in der Summe sind gut $11 \%$ der gesamten Heimkosten

\footnotetext{
${ }^{84}$ errechnet nach Stichting Centraal Administratie Kantoor AWBZ (1993) S. 50 f.
} 
dadurch gedeckt. Der Maximalsatz von $2.200 \mathrm{hfl}$ wird nur in knapp sechs von hundert Fällen entrichtet. ${ }^{85}$

Abb. 5.2

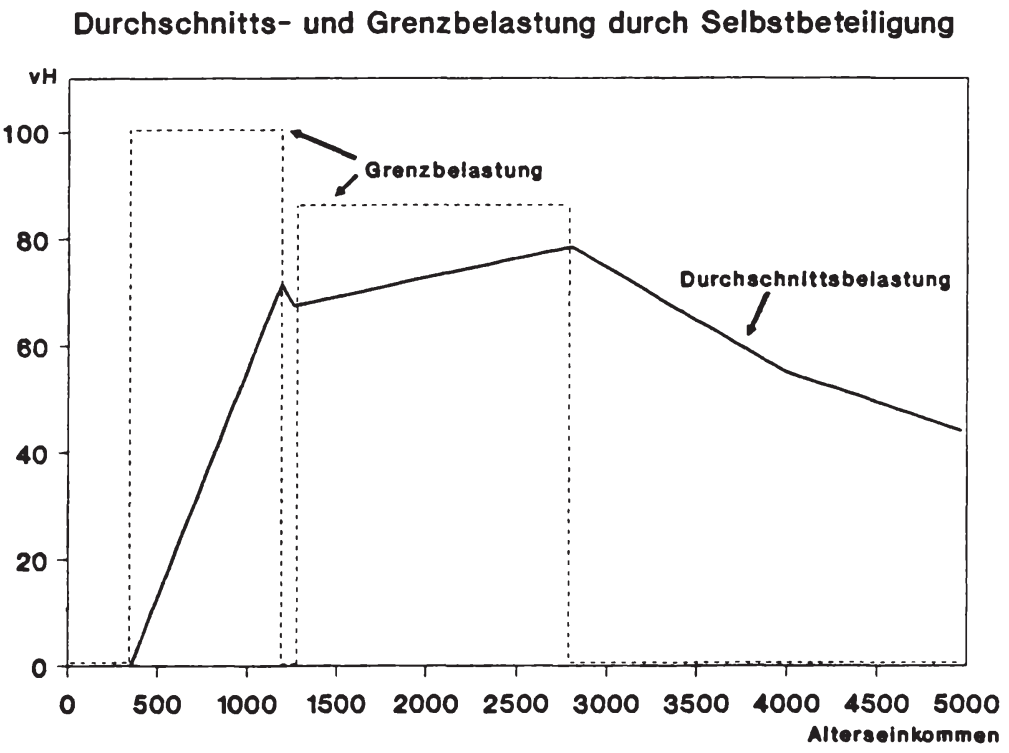

eigene Darstellung

Auch für die ambulanten Dienste hauswirtschaftlicher Art wird ein Eigenbeitrag erhoben, während die ambulante Pflege bislang kostenlos gewährt wird. Er beträgt

${ }^{85}$ ebenda, S. 47 
nun einheitlich 10,- hfl pro Stunde. Die monatliche Gesamtbelastung darf aber bestimmte Grenzen nicht überschreiten, die wiederum einkommensabhängig sind.$^{86}$

\subsection{Die Entwicklung der Leistungsmengen und -preise im Überblick}

\subsubsection{Stationärer Sektor}

Die Gesamtkosten der niederländischen Pflegeheime haben sich - in nominalen Größen zwischen 1972 und 1992 um rd. 478\% erhöht. ${ }^{87}$ Im folgenden gilt es diese Zahl, die auf den ersten Blick die verbreitete Einschätzung einer - mutmaßlich versicherungsbedingten - "Kostenexplosion" zu bestätigen scheint, in Hinblick auf ihre Bestimmungsfaktoren und die Geschichte ihres Zustandekommens zu untersuchen. Von einer Bewertung wird dabei vorläufig weitgehend abgesehen. Sie ergibt sich, auch unter Rückgriff auf Vergleiche mit der Bundesrepublik Deutschland, im Verlauf des sechsten Kapitels.

Zum Zweck einer ersten groben Aufteilung ist festzustellen, daß die reale, d.h. um den allgemeinen Verbraucherpreis-Index bereinigte Kostensteigerung im Zeitraum 1972 - $1989^{88}$ stärker auf die Mengenentwicklung als auf die der Pflegesätze zurückgeht. Letztere sind um 29\% gestiegen, während die Anzahl der Pflegetage um annähernd drei Viertel zugenommen hat. Im folgenden werden beide Komponenten nacheinander behandelt.

\section{a) Kapazitätsentwicklung}

Es ist bereits darauf hingewiesen worden, daß mit der Verabschiedung des AWBZ zunächst ein Ausbau der Pflegekapazitäten angestrebt wurde. Auf Basis von Bedarfsnor-

\footnotetext{
${ }^{86}$ Dies ist der gegenwärtige Stand, nachdem Bemühungen um eine einheitliche Neuregelung der Selbstbeteiligung im ambulanten Sektor vorerst gescheitert sind. Entnommen ist er einer unveröffentlichten Mitteilung des Ministerie van Welzijn, Volksgezondheid en Cultuur an den Ziekenfondsraad vom 8. März 1994.

${ }^{87}$ W. Groenen/R. van der Wouden (1991), S. 109, fortgeschrieben mittels Centraal Administratie Kantoor AWBZ (1993), S. 54f.

${ }^{88}$ Diesen Zeitraum deckt die umfassende Darstellung durch Groenen/van der Wouden (1991) ab. Die Verlängerung ihrer Datenreihen in die jüngere Vergangenheit ist zum Teil nicht bruchlos möglich und erfolgt in den nächsten Abschnitten zumeist separat.
} 
men für die Bettenzahl im Verhältnis zur Stärke der Gesamtbevölkerung wird die Sollkapazität seitens der Regierung zum Einführungszeitpunkt der Pflegeversicherung auf rd. 32.000 Plätze taxiert. ${ }^{89} \mathrm{Zu}$ Beginn des Jahres 1968 stehen 22.300 Betten zur Verfügung..$^{90}$ Auffällig ist dabei, daß bereits diese Zahl das Ergebnis einer rasanten Entwicklung darstellt, die sich in den Jahren vor Einführung abgespielt hat: Schon 1967, also noch unter alten Finanzierungsbedingungen, scheint sich der Bestand an Plätzen gegenüber 1963 verdoppelt zu haben. ${ }^{91}$

Im Jahre 1972 ist das für 1968 fixierte Bedarfsziel mit 33.600 Plätzen erstmals überschritten. ${ }^{92}$ Seitdem ist die Bettenzahl nochmals um gut $50 \%$ auf 51.682 im Jahre 1990 angestiegen, für 1992 wird sie mit knapp 52.200 angegeben. ${ }^{93}$ Knapp drei Viertel des Zuwachses bis 1990 entfallen auf den Zeitraum bis 1980, nach 1985 ist nur noch ein Zuwachs um insgesamt 5,4\% zu verzeichnen gewesen. Das anfangs als "bedarfsgerecht" festgesetzte Ziel an Betten pro 1000 Einwohner (aller Altersgruppen) ergäbe für 1990 einen Normwert von nur 37.300. ${ }^{94}$

Sinnvoller ist es allerdings, die stark veränderte Altersstruktur der Bevölkerung in Rechnung zu stellen. Daher soll hier probeweise eine Fortschreibung der Norm von 1968 unternommen werden, die anhand der Entwicklung derjenigen Altersgruppen erfolgt, die als Bewohner von Pflegeheimen vorrangig in Betracht kommen. Dies sind die über 65jährigen und darin vor allem die über 80jährigen, die mittlerweile $60 \%$ der Heimpopulation stellen. ${ }^{95}$ Bei Verwendung des Mittels aus den Wachstumsraten der 65-79jährigen einerseits und der noch Älteren andererseits als Anpassungsfaktor ergibt sich die in Tabelle 5.1 den jeweiligen Ist-Werten gegenübergestellte Sollentwicklung.

\footnotetext{
${ }^{89}$ Kikkert et al. (1966), S. 5

${ }^{90}$ P.J. Blommestijn (1990), S. 146

${ }^{91}$ Siehe ebenda, S. 137. Blommestijn weist allerdings auf Unsicherheiten in der Datenlage hin.

${ }^{92}$ W. Groenen/R. van der Wouden (1991), S. 111

${ }^{93}$ Tweede Kamer (1994), S. 95

${ }^{94}$ errechnet mit Hilfe von CBS (1991), S. 37

${ }^{95}$ Groenen/van der Wouden (1991), S. 111
} 
Zahl der Plätze in Pflegeheimen: Soll-Ist-Vergleich auf Basis einer Kennzahl für die Entwicklung der Altersbevölkerung

\begin{tabular}{||c|c|c|c|c||}
\hline \multirow{2}{*}{ Jahr } & \multirow{2}{*}{$\begin{array}{l}\text { 65-79jährige } \\
\text { in Tausend }\end{array}$} & \multirow{2}{*}{$\begin{array}{l}\text { üb. 80jährige } \\
\text { in Tausend }\end{array}$} & \multicolumn{2}{|c||}{ Bettenzahl } \\
\cline { 4 - 5 } & & & Soll & Ist \\
\hline 1968 & 1.036 & 212 & 32.000 & 22.300 \\
\hline 1980 & 1.303 & 312 & 43.680 & 46.600 \\
\hline 1985 & 1.359 & 371 & 48.747 & 49.500 \\
\hline 1990 & 1.478 & 428 & 54.645 & 51.600 \\
\hline
\end{tabular}

Quellen: Blommestijn (1990), S. 144; CBS (1991), S. 37 und Groenen/v.d. Wouden (1991), S. 111, eigene Berechnungen

Die Begründung oder Begründbarkeit der anfänglichen Norm kann hier nicht erörtert oder gar beurteilt werden. Vielmehr hat die obige Aufstellung einzig den Sinn zu zeigen, daß die Inanspruchnahme stationärer Pflege der ursprünglichen Zielvorstellung in adäquater Fortschreibung bis in die jüngste Zeit in etwa gefolgt ist. Dies unbeschadet anders definierter Normschlüssel, wie sie in der Zwischenzeit verwendet worden sind. ${ }^{96}$ BLOMMESTIJN ${ }^{97}$ kommt bei seiner detaillierten Analyse dieser Richtlinien für den Zeitraum bis $1985 \mathrm{zu}$ dem Ergebnis, daß sie erstens seit den 60er Jahren eine weitgehende Kontinuität wahren und zweitens durchweg eingehalten bzw. nur leicht überschritten worden sind. Diese These kann durch die oben angestellte Rechnung als gedeckt gelten.

Bei genauer Betrachtung der in Tabelle 5.1 nur grob skizzierten Entwicklung läßt sich eine - wenn auch unspektakuläre - Trendwende zu Beginn der 80er Jahre ablesen. War bis 1980 ein kontinuierlicher Anstieg der Ist/Soll-Relation zu verzeichnen, so geht sie seither zurück; bis 1990 hat sich erneut eine Ist-Abweichung nach unten ein-

\footnotetext{
96 siehe etwa Kluwer Zorg en Dienstverlening (1990), S. 3

${ }^{97}$ P.J. Blommestijn (1990), S. 137, 146 und 148
} 
gestellt; der stationäre Versorgungsgrad hat - bezogen auf beide relevanten Altersgruppen - abgenommen. ${ }^{98}$ Die Wende fällt in etwa zusammen mit der Erweiterung des Leistungskatalogs im AWBZ um die häusliche Pflege und markiert daher wohl einen Wechsel im Leitbild der Pflegepolitik: Wurde bis dahin vorrangig das Ziel verfolgt, eine als mangelhaft empfundene Versorgung von der stationären Angebotsseite her zu verbessern, tritt nunmehr die Idee in den Vordergrund, den Bedarf an Heimplätzen durch die Förderung ambulanter Alternativen zu dämpfen.

\section{Tabelle 5.2}

Wachstumsraten der Pflegeheimkapazität und der Bevölkerung über 79 Jahre in den Niederlanden (in Prozent)

\begin{tabular}{|c|c|c|c|c|}
\hline \multirow{2}{*}{ Zeitraum } & \multicolumn{2}{|c|}{ Zunahme Pflegebetten } & \multicolumn{2}{|c|}{ Zunahme Hochbetagte } \\
\hline & gesamt & p.a. & gesamt & p.a. \\
\hline 1980-1989 & 9,4 & 1,0 & 37 & 3,6 \\
\hline 1990-1994 & $12,3^{*}$ & 2,9 & -- & $(1,9)^{* *}$ \\
\hline \multicolumn{5}{|c|}{$\begin{array}{ll}\text { *) } & \text { teils geplant } \\
\text { **) } & \text { durchschnittl. jährliche Zunahme in den Jahren } 1990 \\
& \text { und } 1991\end{array}$} \\
\hline
\end{tabular}

Quellen: $\quad$ CBS (1993), S. 35, Groenen/v.d. Wouden (1991), S. 111, Tweede Kamer (1994), S. 13f., eigene Berechnungen

In jüngster Zeit allerdings scheint sich unter dem Eindruck von Wartelisten zunehmender Länge die Einschätzung durchzusetzen, daß die Heimpflege auf diesem Wege keineswegs unbegrenzt substituierbar ist. Ein beschleunigter Ausbau der Kapazitäten wird für erforderlich gehalten und derzeit vollzogen: In der Zeit von 1990 bis 1994 sollen insgesamt über 6.200 zusätzliche vollstationäre Plätze geschaffen werden, "um

${ }^{98} \mathrm{vgl}$. Groenen/van der Wouden (1991), S. 110 
Defizite abzuarbeiten". ${ }^{99}$ Gleichzeitig wird die Anzahl der Tagespflegeplätze nochmals kräftig aufgestockt. Der angestrebte vollstationäre Ausbau ist in diesem Zeitraum prozentual stärker als in der etwa doppelt so langen Periode von 1980 bis 1989. In bezug auf die demographische Lage tritt der Richtungswechsel noch deutlicher hervor, da die Anzahl der Hochbetagten als der wesentlichen Zielgruppe derzeit offenbar deutlich langsamer wächst als in den Jahren zuvor. Daher nimmt die Relation zwischen Heimplätzen und Altersbevölkerung im Augenblick offenbar erstmals seit den 70er Jahren wieder leicht zu. Tabelle 5.2 zeigt den Zusammenhang.

b) Kostenentwicklung

Die Entwicklung der Kosten pro Pflegetag zeigt ebenfalls beträchtliche Steigerungsraten. Wirklich brauchbare und interpretationsfähige Daten liegen über den Zeitraum ab 1972 vor, dennoch sei die Zeit davor mittels einiger nominaler Daten zur Orientierung zunächst grob umrissen:

In den Parlamentsunterlagen anläßlich der Verabschiedung des AWBZ nennt der Sozialminister für 1965 Pflegesätze in der Größenordnung von 25 hfl pro Tag. ${ }^{100}$ Sofern diese Zahl als kompatibel mit den bei $\mathrm{KESS}^{101}$ genannten gelten kann, ist bereits bis zum Einführungsjahr der Pflegeversicherung 1968 ein Anstieg um rd. 50\% auf gut $37 \mathrm{hfl}$ zu verzeichnen, dem bis 1972 eine Verdoppelung folgt.

Über den Hintergrund dieser raschen Steigerung läßt sich angesichts fehlender disaggregierter Daten nur vermuten, daß er in erster Linie in einer kräftigen Personalentwicklung besteht. ${ }^{102}$ Jedenfalls ist im Jahre 1972 mit 0,93 Arbeitsplätzen pro Patient ${ }^{103}$ bereits ein hoher Wert erreicht, der in den Folgejahren nur noch geringfügig ansteigen wird und mit den extrem niedrigen Kostensätzen der 60er Jahre nicht vereinbar erscheint.

\footnotetext{
99 Tweede Kamer (1994), S. 13f.

${ }^{100}$ siehe Kikkert et al. (1966), S. 5

101 W. Keß (1980), S. 382

102 ähnlich Keß (1980), S. 383

${ }^{103}$ Groenen/van der Wouden (1991), S. 112
} 
Zwischen 1972 und 1980 sind die nunmehr realen Kosten pro Tag nochmals um 30\% gestiegen, dann zunächst gesunken und nach 1985 wieder ganz langsam gewachsen. Der Knick zu Beginn der 80er Jahre ist zum Teil auf die erwähnte Budgetierung mit ihrem stark und nachhaltig dämpfenden Effekt auf die Sachkosten zurückzuführen. Im Jahre 1990 ist der bisherige reale Höchststand von 1980 erstmals überschritten und eine neue Phase von Kostensteigerungen von real zuletzt 1,5\% p.a. eingeleitet worden. ${ }^{104}$ Im Jahre 1992 beträgt der durchschnittliche Tagessatz im Pflegeheimen rd. 245 hfl. Abbildung 5.3 zeigt die Entwicklung des Pflegesatzes und seiner Komponenten im Überblick.

Abb. 5.3

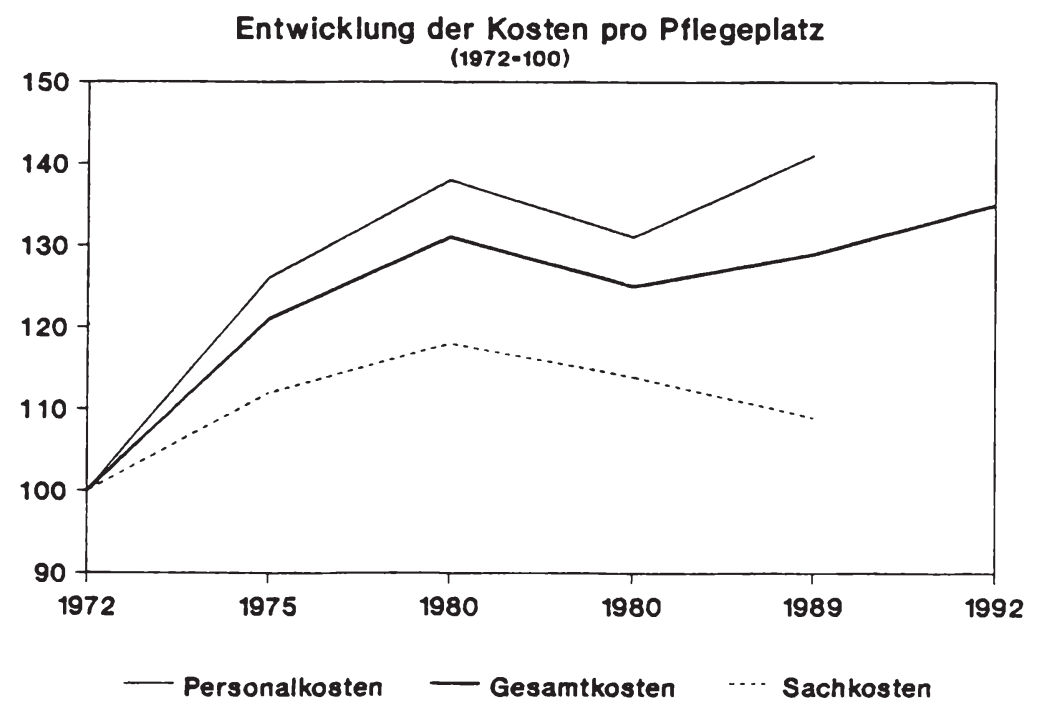

Eigene Darstellung anhand von Groenen/v.d.Wouden (1991) und Stichting Centraal Administratie Kantoor AWBZ (1993)

${ }^{104}$ errechnet auf Basis von Stichting Centraal Administratie Kantoor AWBZ (1991) und (1993), S. 38f. bzw. 54f. sowie mithilfe der Verbraucherpreis-Indizes bei CBS (1993), S. 338.

142 
Weitgehend bestimmt wird der Gesamtkostenverlauf von den Personalkosten, die gegenwärtig rd. 70\% ausmachen. Sie sind im Gesamtzeitraum deutlich rascher gestiegen als die übrigen Komponenten. Dies geht zum geringeren Teil auf die langsam zunehmende Anzahl Beschäftigter pro Pflegeplatz, zum größeren Teil darauf zurück, daß die durchschnittlichen Jahreslöhne stark gewachsen sind. Im Zeitraum 1975 bis 1980 war eine Abkoppelung dieser Größe von der allgemeinen Lohnentwicklung nach oben zu beobachten. Von Bedeutung dürfte dabei auch eine veränderte Zusammensetzung des Personals in Richtung auf höhere Qualifikationsniveaus gewesen sein. ${ }^{105}$ In den frühen 80er Jahren - also etwa zeitgleich mit dem Budgetierungseffekt - kehrte sich dieser Trend und damit auch die Entwicklung der realen Lohnkosten vorübergehend um, was mit einer Verlängerung der Lehrzeit für Pflegeberufe zusammenhing. Der Anteil niedrig entlohnter Auszubildender am Personalbestand ist dadurch angestiegen. ${ }^{106}$ Anders als die Sachkosten sind die Personalkosten pro Pflegeplatz aber schon ab Mitte der 80er Jahre auf einen Wachstumspfad zurückgekehrt.

\subsubsection{Ambulanter Sektor}

Da die ambulanten Dienste erst später als die stationären in das System der Pflegeversicherung aufgenommen worden und nach meinen Erkenntnissen insgesamt weniger vollständig statistisch erfaßt sind, können hier nur vergleichsweise kurze Reihen gebildet werden. Für das Kruiswerk, also die mit der häuslichen Pflege im engeren Sinn betraute Organisation, läßt sich die Entwicklung der Gesamtkosten einschließlich der Verwaltungskosten und der Kosten für Beratungs- und Präventionsprogramme nur für den Zeitraum 1984 - 1991 nachzeichnen (Tabelle 5.3), wobei allerdings die nicht zulasten der Versicherung abgerechneten Anteile enthalten sind, die im wesentlichen aus Mitgliedsbeiträgen stammen. Ihr Umfang ist mir nur für das Jahr 1987 bekannt und betrug damals $14,6 \% .{ }^{107}$

Es zeigt sich für den betrachteten Zeitraum ein recht gleichmäßiger Anstieg von nominal 5,1\% p.a. Etwa 1,2 Prozentpunkte davon gehen auf die Entwicklung des

\footnotetext{
${ }^{105}$ vgl. Groenen/van der Wouden (1991), S. 116

${ }^{106}$ So verstehe ich angesichts der dort verwandten Quelle die etwas unklaren Ausführungen bei Groenen/van der Wouden (1991), S. 113f.

${ }^{107}$ Errechnet aus Angaben bei Nationale Kruisvereniging (1989), S. 27.
} 
allgemeinen Verbraucherpreisindex zurück. ${ }^{108}$ Die Zerlegung des realen Anstiegs von demnach etwa 3,9\% im Durchschnitt pro Jahr in eine Mengen- und eine Preiskomponente kann nur in recht grober Näherung erfolgen, da

- die nicht auf die eigentliche Pflegeaktivität entfallenden Kosten für Beratung und Prävention nicht herausgerechnet werden können,

- $\quad$ keine Angaben darüber vorliegen, ob die statistisch erfaßten Klienten-Kontakte (Besuche) in allen Berichtsjahren den gleichen mittleren Zeitaufwand erfordert haben und zudem

- die Anzahl dieser Kontakte nicht absolut, sondern nur in Relation zur Bevölkerung angegeben wird. Der Versuch, die entsprechenden Quoten mit Bevölkerungsdaten zu multiplizieren, dürfte zu Ungenauigkeiten führen.

Tabelle 5.3

Gesamtkosten Kruiswerk (häusliche Pflege) 1984 bis 1991

\begin{tabular}{|c|c|}
\hline Jahr & Mio. hfl (laufende Preise) \\
\hline \hline 1984 & 713 \\
\hline 1985 & 754 \\
\hline 1986 & 806 \\
\hline 1987 & 841 \\
\hline 1988 & 880 \\
\hline 1989 & 909 \\
\hline 1990 & 970 \\
\hline 1991 & 1011 \\
\hline
\end{tabular}

Zusammengestellt aus: NKV (1989), S. 27, LVT (1993), S. 41

${ }^{108}$ ermittelt nach CBS (1993), S. 338 
Unter diesen Vorbehalten ergibt sich folgendes Bild vom Anstieg in der Anzahl an "Pflegekontakten": 109

$$
\begin{aligned}
& \text { 1985: 1,04 Mio Besuche } \\
& \text { 1988: 1,06 Mio Besuche } \\
& \text { 1990: 1,16 Mio Besuche } \\
& \text { 1991: 1,20 Mio Besuche }
\end{aligned}
$$

Die Steigerung von gut $15 \%$ liegt oberhalb der Zunahme der Bevölkerung ab 65 Jahren im gleichen Zeitraum $(+11,8 \%)$, aber unterhalb derjenigen der über 79jährigen als der bedeutendsten Zielgruppe ${ }^{110}(+18 \%)$. In dieser Betrachtung ${ }^{111}$ kann der Versorgungsgrad im erfaßten Zeitraum demnach als ungefähr konstant bezeichnet werden.

Auf Basis dieser Mengendaten läßt sich über die Preisentwicklung folgendes aussagen: Pro Besuch und in laufenden Preisen haben die Gesamtkosten des Kruiswerk zwischen 1985 und 1991 um 16,2\% zugenommen, die reale Steigerung beträgt rd. 7\% bzw. $1,15 \%$ pro Jahr. Der Grund dafür liegt auch hier offenkundig in steigenden Reallöhnen bei sehr hohem Personalkostenanteil und weitgehend fehlenden Rationalisierungsspielräumen. ${ }^{112}$

Für die hauswirtschaftlich orientierte Hilfe schließlich sind nur die Jahre 1988 - 1991 dokumentiert; damit ist der Zeitpunkt eingeschlossen, zu dem die Finanzierung im wesentlichen auf die Pflegeversicherung übergegangen ist (1. Januar 1989). Auch hier liegen nur Globalgrößen vor, die in diesem Fall neben den Eigenbeiträgen der Hilfeempfänger auch einen (kleineren) Posten enthalten, der zulasten der Invaliditätsversicherung abgerechnet wird. ${ }^{113}$ Nominal sind diese Kosten in drei Jahren um $15,7 \%$ gestiegen und haben ein Niveau von 1,943 Mrd Gulden erreicht. ${ }^{114}$ Etwa

\footnotetext{
${ }^{109}$ Errechnet aus Angaben bei NKV (1989), S. 14; LVT (1993), S. 39 und CBS(1991 und 1993), S. 37 bzw. 35 .

${ }^{110}$ Auf sie entfallen inzwischen über $45 \%$ aller Besuche (LVT (1993), S. 40).

${ }^{111}$ D.h. ohne Berücksichtigung der Verteilung der Besuche auf die Empfänger, siehe dazu Kap. 6.

${ }^{112}$ Zur Illustration: Die Arbeitskosten pro Industriearbeiterstunde sind in den Niederlanden zwischen 1985 und 1991 mit gut $18 \%$ etwa so stark gestiegen wie die Pflegekosten pro Besuch (siehe CBS (1993), S. 129).

113 siehe LVT (1993), S. 10

114 vgl. LVT (1993), S. 41
} 
die Hälfte des Anstiegs entspricht der allgemeinen Preisentwicklung, der reale Anstieg pro Jahr beträgt $2,4 \%$.

Das ist insofern auffällig, als den verfügbaren Daten zufolge keine Ausweitung des Einsatzvolumens in Stunden stattgefunden hat. ${ }^{115}$ Vermutlich sind die - bei niedrigem Qualifikationsniveau der Beschäftigten traditionell recht geringen - Lohnsätze hier etwas stärker angestiegen als beim Kruiswerk. Jedenfalls wird von Schwierigkeiten bei der Rekrutierung von Personal berichtet. ${ }^{116}$ Daneben könnte eine Rolle spielen, daß die Anzahl der betreuten Personen - bei entsprechendem Rückgang der Einsatzstunden pro Kopf - deutlich zugenommen hat. Es ist davon auszugehen, daß pro "Fall" in gewissem Umfang Fixkosten auftreten.

\subsection{Aktuelle Tendenzen und Perspektiven künftiger Entwicklung}

\subsubsection{Die Reform des Krankenversicherungswesens in den Niederlanden}

Das System der Krankenversicherung in den Niederlanden ist seit Ende der 80er Jahre Gegenstand von Bemühungen um eine tiefgreifende Reform in Richtung auf mehr Wettbewerb. ${ }^{117}$ Der Bereich der Pflege und deren Sicherung stehen dabei nicht eigens im Mittelpunkt der teils schon vollzogenen, teils geplanten Veränderungen, sind aber zum Teil (potentiell) mitbetroffen. Dies gilt sowohl für die Finanzierungsseite als auch für die Definition, Erstellung und Abrechnung der Leistungen. Da der Reformprozeß einerseits schon erkennbar eingesetzt hat, andererseits inzwischen in einem Gewirr politischer Auseinandersetzungen, akuter finanzieller Zwänge sowie in einer Fülle von Detail- und Übergangsproblemen zu versanden droht, ist eine äußerst unübersichtliche und instabile Lage entstanden. In den Niederlanden ist dafür der ebenso vornehme wie treffende Begriff der "semipermanenten Übergangsphase" geprägt worden. ${ }^{118}$

Nach dem Regierungswechsel im Jahre 1994 ist das Scheitern der bisherigen Ablaufplanung auch offiziell festgestellt worden. Da gleichzeitig eine Rückkehr zum Zustand

\footnotetext{
${ }^{115}$ Grob, weil auf Umwegen ermittelt aus Angaben ebenda, S. 11.

${ }^{116}$ siehe ebenda, S. 30

${ }^{117}$ Für einen deutschsprachigen Überblick über die Reform(pläne) nach damaligem Stand siehe J.Müller (1990). Einschlägige Beiträge auf Englisch sind W.P.M.M. van de Ven (1990) und OECD (1992), Kap. 7

${ }^{118}$ so der Ziekenfondsraad 1t. P.A.M. van den Broek (1993), S. 950
} 
vor Beginn der Reformbemühungen unvorstellbar ist, findet nunmehr offenbar eine Neubesinnung in bezug auf den Reformpfad statt, über deren Ergebnisse gegenwärtig (Anfang 1995) bestenfalls spekuliert werden könnte.

Die bisher ausgeklammerten (möglichen) Folgen der Reform für den Bereich der Pflege und der Pflegeversicherung werden nunmehr auf dem Hintergrund eines kurzen Überblicks über den Ursprung, das Konzept und den aktuellen Stand der Reform gesondert abgehandelt.

\subsubsection{Das Reformprojekt im Überblick: Gründe, Ziele, Konzepte}

Das herkömmliche System der Krankenversicherung in den Niederlanden steht neben dem für die Pflege und andere Bereiche zuständigen AWBZ und ist dem deutschen in seinen Grundzügen recht ähnlich: Ebenso wie dieses zerfällt es in einen gesetzlich organsierten und einen privaten Zweig, wobei über die Zuordnung des einzelnen Versicherten bei abhängig Beschäftigten die Höhe des Lohneinkommens entscheidet. Aufgrund einer niedrigeren Versicherungspflichtgrenze und der fehlenden Möglichkeit zur freiwilligen Mitgliedschaft in der $\mathrm{GKV}^{119}$ ist der privat versicherte Bevölkerungsanteil in den Niederlanden mit fast $40 \%^{120}$ allerdings weitaus höher als in der Bundesrepublik. Für Beamte besteht dort wie hier ein eigenes Versorgungswesen.

Bilden sich die Prämien im privaten Sektor grundsätzlich ${ }^{121}$ am Markt, so entrichten die gesetzlich Versicherten einen bestimmten Anteil ihres Erwerbseinkommens. Etwa zwei Drittel des Beitrags werden dabei vom Arbeitgeber abgeführt. ${ }^{122} \mathrm{Im}$ Unterschied zur Bundesrepublik Deutschland ist der Beitragssatz landesweit einheitlich, was bis vor kurzem einen vollständigen Ausgabenausgleich zwischen den Kassen bedingte. Aus ökonomischer Sicht kann mithin von einer faktischen Einheitskasse gesprochen

\footnotetext{
119 J. Müller (1990), S. 198

${ }^{120}$ W.P.M.M. van der Ven (1990), S. 635

${ }^{121}$ Das heißt hier: mit Einschränkungen. Den privaten Versicherern ist zur Vermeidung untragbarer Prämien für Gruppen mit erhöhtem Schadensrisiko zur Vorschrift gemacht worden, einen speziellen Standardtarif anzubieten, wie dies neuerdings auch in der Bundesrepublik für privat versicherte Rentner vorgesehen ist. Die dabei auftretende Deckungslückewird durch Prämienaufschläge zulasten der übrigen Privatversicherten geschlossen. Siehe zu diesem Verfahren und seinen Problemen etwa F.T Schut/R.C.J.A. van Vliet (1992).
}

122 Stand 1991; siehe Tweede Kamer (1991), S. 45 
werden, zu der sich die formal selbständigen Einzelkassen wie bloße Ausführungsorgane verhalten. ${ }^{123}$ Diese Struktur entspricht somit derjenigen der Pflegeversicherung mit dem Unterschied, daß dort auch private Kassen in gleicher Weise als Ausführungsagenturen fungieren.

Da ein Anreiz zu wirtschaftlichem Verhalten unter diesen Umständen nicht besteht, versucht der Staat, Mengen und Preise der medizinischen Leistungen durch ein dichtes Netz von Regulierungen unter Kontrolle zu halten. Die Wirkungsweise der einschlägigen Gesetze über die Bereitstellung von stationären Kapazitäten und über die Leistungstarife ist in bezug auf den Pflegesektor bereits skizziert worden. Beide Regelwerke gelten auch für solche Leistungsbereiche, die von der Krankenversicherung abgedeckt werden.

Die Reformbestrebungen antworteten auf ein wachsendes Unbehagen an diesem Gefüge, das als ineffizient, inflexibel und zudem als ungerecht empfunden wird. Neben unzureichenden Anreizen innerhalb des gesetzlichen Sektors wurden auch Koordinierungsprobleme zwischen diesem und der PKV sowie zwischen Kranken- und Pflegeversicherung als Gründe für eine Neuordnung geltend gemacht. Im Hinblick auf die Privatversicherungen wurden eine Tendenz zum Ausschluß ungünstiger Kunden und die inkonsistente Verteilung der Beitragslasten bemängelt, wie sie sich aus dem Nebeneinander der Systeme ergibt. ${ }^{124}$

Gesucht war ein neues geschlossenes Gesamtkonzept, das unter Wahrung des allgemeinen und gleichen Zugangs zu hochwertigen Gesundheitsleistungen wirtschaftliches Verhalten der Beteiligten fördern, Wahlmöglichkeiten erweitern und die Finanzierungslast gleichmäßig verteilen sollte. ${ }^{125}$ Auch eine erhöhte Zielgenauigkeit der Versorgung und ein spürbarer Abbau dirigistischer Elemente gehören zum Zielkatalog der Reform, die überdies eine verbesserte "Beherrschung" der Gesamtkosten ermöglichen soll. ${ }^{126}$

\footnotetext{
Wettlaufs um die großzügigsten Leistungen hätte annehmen können.

${ }^{124}$ vgl. van der Ven (1990), S. 634ff.

${ }^{125}$ vgl. Commissie Structuur en Financiering Gezondheidszorg (1987), S. 5ff.

${ }^{126}$ vgl. Ziekenfondsraad (1993), S. $80 f$.
}

${ }^{123}$ Es existierten zu Beginn der Reform 40 sogenannte "Ziekenfondsen", die durch regionale Abgrenzung nicht in Konkurrenz zueinander standen (vgl. van der Ven (1990), S. 635). Das ergibt sich fast zwingend aus den genannten Bedingungen, unter denen ein Wettbewerb allenfalls die Form eines ungehemmten 
Eine von der Regierung eingesetzte Kommission präsentierte erstmals 1987 Vorschläge zu einer Reorganisation, an deren Grundzügen sich das Reformprojekt bis heute orientiert. Als wesentlichste Elemente vorgesehen sind dort: ${ }^{127}$

- die Schaffung einer die gesamte Bevölkerung umfassenden "Basisversicherung" unter Aufhebung der systematischen Trennung zwischen öffentlichem und privatem Versicherungswesen. Bei uneingeschränkter ${ }^{128}$ Wahlfreiheit aller Versicherungsnehmer sollen Anbieter aus beiden bisher getrennten Sektoren in Wettbewerb miteinander treten.

- die Ersetzung des vollständigen Ausgabenausgleichs zwischen den gesetzlichen Kassen durch einen Risikostrukturausgleich zwischen allen Versicherern. Die zentral eingezogenen Beitragsmittel sollen in Gestalt sogenannter "Normzuwendungen" nach Maßgabe von Risikomerkmalen ihrer Kunden an die einzelnen Versicherer verteilt werden.

- eine für alle Versicherten strukturell einheitliche Verteilung der Finanzierungslast: Neben dem einkommensproportionalen Beitrag, der durch eine Bemessungsgrenze nach oben limitiert ist, wird eine - vergleichsweise geringe sogenannte "nominale Prämie" erhoben. Diese ist für alle Kunden einer Versicherung ohne Ansehen von Risikomerkmalen zunächst absolut gleich; variieren kann sie hingegen zwischen den Kassen sowie in Abhängigkeit davon, ob der einzelne Versicherte einen Teil des Risikos freiwillig selbst übernimmt. Daß eine solche Möglichkeit zur Wahl eines Tarifs mit Selbstbeteiligung besteht und wahrgenommen wird, ist im Sinne der angestrebten Ausweitung von Wahlfreiheit und Eigenverantwortung ausdrücklich erwünscht. ${ }^{129}$

Damit sich ein Wettbewerb zwischen den Krankenversicherungen vermittels der einkommensunabhängigen Prämie tatsächlich und in volkswirtschaftlich sinnvoller Weise entfalten kann, sollen die bislang durch Kartellstrukturen und staatliche Regulierung gekennzeichneten und daher kaum differenziert gestaltbaren Beziehungen zwischen den Kassen und den Anbietern medizinischer Leistungen neu bestimmt werden. Durch die Schaffung weitgehender Vertragsfreiheit zwischen Akteuren beider Seiten sollen

\footnotetext{
${ }^{127}$ referiert nach P.A.M. van den Broek (1993), S. 874ff.

128 Dies impliziert auf seiten der Versicherer eine Annahmepflicht gegenüber allen, die um Versicherungsschutz nachsuchen.
}

${ }^{129}$ vgl. F.M. Bakker et al. (1993), S. 1105 
marktgerechte Preise sichergestellt und ein Druck in Richtung auf innovatives Verhalten erzeugt werden. Dem letzteren Ziel dient auch die vorgesehene Ersetzung aller auf bestimmte Institutionen bezogenen Leistungsansprüche durch rein funktionale Umschreibungen. Gerade in Bereichen wie dem der Pflege soll so die Substitution teurer durch kostengünstige und dennoch bedarfsgerechte Betreuungsformen gefördert werden.

\subsubsection{Zum Stand und Stillstand der Reform}

Um den Übergang auf das neue System schrittweise zu vollziehen, sollten die Leistungsbereiche der Krankenversicherung nach und nach auf das AWBZ übertragen werden, das dazu als ungeteilte Volksversicherung prädestiniert erschien. Die dort bereits vorhandene Zentralkasse, in der die mit den Steuern erhobenen Beiträge gesammelt werden, sollte so in ihre neue umfassende Funktion hineinwachsen. Umzustellen wäre dann im oben beschriebenen Sinn das Verfahren, nach dem die Mittel von dort aus den einzelnen Versicherungen zufließen.

Nachdem der Arzneimittelbereich und einige kleinere Posten zum Jahresbeginn 1992 in der Tat auf das AWBZ übergegangen sind, ist der Prozeß des gleitenden Übergangs zum Stillstand gekommen. Die für Anfang 1993 ins Auge gefaßte Übertragung der hausärztlichen Leistungen ist nicht erfolgt. ${ }^{130}$ Nach einiger Zeit großer Unklarheit besagen neueste Meldungen, die zunächst verfolgte Reformstrategie sei nunmehr "vom Tisch"; die in das AWBZ übertragenen Leistungsbereiche sollen demnächst in die Krankenversicherung zurückkehren. ${ }^{131}$ Als Alternative zum Reformweg über das AWBZ ist vorgeschlagen worden, private und gesetzliche Kassen in ihren heutigen Grenzen zunächst fortbestehen zu lassen und separat umzugestalen. ${ }^{132}$ Die Vereinheitlichung des Finanzierungssystems würde nach dieser Vorstellung am Schluß des Verfahrens in einem Zug erfolgen.

Auf solche Varianten und ihre schwer durchschaubaren politischen Hintergründe braucht hier nicht näher eingegangen zu werden. Offenbar sind es nicht allein Detailfragen

\footnotetext{
${ }^{130}$ siehe N. de Boer (1992), S. 10

${ }^{131}$ siehe Trouw vom 13. August 1994: Eigen risico voor iedereen

${ }^{132}$ Siehe dazu W.P.M.M. van de Ven (1992). Als Schritt in dieser Richtung wird dort (S. 15) zum Beispiel angesehen, daß private Versicherer inzwischen bereits in großem Umfang verpflichtet sind, interessierte Kunden zu gesetzlich gedeckelten Prämien zu akzeptieren.
} 
des Übergangs zum neuen System, die den Fortgang der Reform hemmen. Vielmehr ist mittlerweile auch die Zielvorstellung selbst in manchen Punkten undeutlich geworden. Angesichts großer Verzögerungen und Unstimmigkeiten im Ablauf der Reformschritte ist die Diskussion um die Eckpunkte des Programms insgesamt neu aufgeflammt ${ }^{133}$ und das Vertrauen der Öffentlichkeit und der Akteure auf ein Gelingen der Reform wird brüchig. ${ }^{134}$ In letzter Instanz hat die vielbeklagte Stockung der anfangs mit viel Schwung betriebenen Umgestaltung wohl politische Gründe, die hier nicht adäquat durchleuchtet werden können. Gleichwohl erscheint es aufschlußreich, die gegenwärtigen Schwierigkeiten in der Sache kurz darzulegen.

Als Teilgebiete der Reformlandschaft lassen sich

- die Beziehungen der Versicherer zu den Leistungsanbietern in seiner Wirkung auf den Wettbewerb zwischen diesen (a),

- das (Konkurrenz-)Verhältnis zwischen den Versicherungen selbst (b) und

- die Rolle des Staates (c)

benennen. Die Problematik erwächst dabei gerade daraus, daß alle drei Aspekte in enger Wechselbeziehung stehen:

ad a): Wettbewerb zwischen Leistungsanbietern

Formal ist die hergebrachte Kontrahierungspflicht der Versicherer gegenüber den Leistungsanbietern 1992 entfallen. ${ }^{135}$ Grundsätzlich obliegt es seitdem den Versicherern, die Leistungsbedingungen und -tarife in eigener Verantwortung auszuhandeln, wobei allerdings Höchstpreise gesetzlich fixiert sind. Die Wirkung dieser an sich sehr bedeutsamen Veränderung ist bislang gering geblieben. Dies liegt teilweise daran, daß alte Verträge noch bis Ende 1993 in Kraft waren, so daß eine umfassende Evaluation der neuen Regelung noch nicht vorgenommen werden kann. ${ }^{136}$ Es zeigen sich aber auch dort, wo eine solche Bindung nicht besteht, Hindernisse auf dem Weg zu einem marktwirtschaftlichen Preisfindungsgeschehen.

\footnotetext{
${ }^{133}$ siehe den Überblick bei C.A. de Kam (1993), S. $74 \mathrm{ff}$.

${ }^{134} \mathrm{vgl}$. Ziekenfondsraad (1993), S. 101

${ }^{135}$ siehe Ziekenfondsraad (1993), S. 50

${ }^{136} \mathrm{vgl}$. ebenda, S. 51
} 
Zum einen treten die Leistungsanbieter traditionell nicht einzeln, sondern in Form von Interessenvertretungen auf, die teils national, teils regional organisiert sind. Gerade im Fall regionaler Verhandlungen erweist sich ihre Position als weitaus stärker als die von Versicherern, die in der einzelnen Region oft nur einen kleinen Versichertenbestand einbringen. ${ }^{137}$

Zum zweiten fehlt es offenbar an wirksamen Anreizen, die spezifischen Verträge tatsächlich abzuschließen. Da es nämlich nicht zulasten der Versorgungssicherheit für die Versicherten gehen soll, wenn eine Vereinbarung nicht zustande kommt, tritt in solchen Fällen eine Art staatlich überwachter Schiedsregelung in Kraft, durch die ein Standardpreis festgesetzt wird. Die ursprünglich vorgesehene Bestimmung, diesen Preis betont niedrig anzusetzen, wodurch das Interesse der Anbieter von Gesundheitsleistungen an Vertragsabschlüssen systematisch verstärkt worden wäre, hat sich im Parlament nicht durchsetzen lassen. ${ }^{138}$ Aber auch auf seiten der Versicherer ist das Interesse an der Erzielung günstiger Leistungspreise mitunter noch unterentwickelt. Der mutmaßliche Grund dafür ergibt sich aus Punkt (b).

Schließlich ist anzumerken, daß die stationären Einrichtungen nach wie vor weitgehend den alten, staatlich dominierten Regelungen unterliegen. Als Richtschnur dient hier weiterhin das in bezug auf die Pflegeheime erwähnte Budgetsystem, wodurch eine freier differenzierte Vertragsgestaltung weitgehend ausgeschlossen bleibt. ${ }^{139}$

Es ist angesichts der hier skizzierten Lage nachvollziehbar, daß mangelnde Fortschritte bei der Erzeugung echter Konkurrenz zwischen den Leistungsanbietern mitunter als zentraler "Engpaß" der Gesundheitsreform eingestuft werden. ${ }^{140}$

ad b): Wettbewerb zwischen Versicherern

Auch in Hinblick auf den angestrebten Kassenwettbewerb ist formal bereits ein entscheidender Schritt vollzogen worden: Seit Anfang 1993 erfolgt die Zuweisung der Beitragsmittel an die Versicherer nicht mehr anhand historischer Kosten, sondern

\footnotetext{
${ }^{137}$ vgl. ebenda, S. 68

${ }^{138}$ siehe ebenda, S. 71

${ }^{139} \mathrm{vgl}$. ebenda, S. 65

${ }^{140}$ vgl. etwa P.A.M. van den Broek, S. 953
} 
nach dem Altersprofil und der Geschlechtsrelation des jeweiligen Versichertenbestandes. Dies gilt für den gesamten Leistungsbereich der Krankenversicherung und einen Teil des AWBZ. ${ }^{141}$ Ebenfalls schon eingeführt sind die sogenannten "nominalen Prämien", die direkt vom Versicherten an seine Krankenkasse abgeführt werden. Über deren variable Höhe sollen die Versicherer miteinander konkurrieren.

Die erwünschte Wirkung hat sich indes auch auf diesem Feld nicht eingestellt. De facto nämlich wird die Anreizwirkung der im voraus zugemessenen Budgets durch nachträgliche Ausgleichsmechanismen weitgehend wieder aufgehoben: In einem ersten Schritt werden zu 75\% die Abweichungen der wirklich getätigten Ausgaben vom normierten Durchschnitt durch Zahlungen der Versicherungen untereinander eingeebnet. Sodann erfolgt ein "Nachschlag" aus der zentralen Beitragskasse, wenn die tatsächlichen Ausgaben eines Versicherers das vorher festgesetzte Budget überschritten haben. Hier beträgt die Quote $85 \%$ der Diskrepanz. Im Endeffekt verbleibt also nur ein kleiner Bruchteil der Normabweichung beim Versicherer selbst. Die Wirkung des Verfahrens ${ }^{142}$ illustriert das folgende Zahlenbeispiel:

\section{Versicherer A Versicherer B}

Budget

Schäden

1. Ausgleich, Durchschnitt Abweichung Zahlung $75 \%$ ( $A$ an $B$ ) Lasten im Resultat

2. Ausgleich Budgetabweichung Zahlung $85 \%$ vom staat Lasten im Endeffekt

$\begin{array}{rrr}100 & & 100 \\ 90 & & 130 \\ & 110 & \\ -20 & & +20 \\ +15 & & -15 \\ 105 & & 115\end{array}$

$\begin{array}{cc}5 & 15 \\ -4,25 & -12,75 \\ 100,75 & 102,25\end{array}$

(Un-)Wirtschaftliches Gebaren wird demnach nur in kleiner Münze belohnt (bestraft). Hintergrund dieser merkwürdig unentschlossenen Regelung ist zum einen die Diskussion um die korrekte Bemessung der normierten Zuwendungen. Die Beschränkung auf Alter und Geschlecht wird nicht nur von den betroffenen Versicherern als viel zu grob

\footnotetext{
${ }^{141}$ Der alte Kern des AWBZ und damit fast alle Pflegeleistungen sind einstweilen noch nicht betroffen (vgl. Ziekenfondsraad (1993), S. 40f.).

${ }^{142}$ siehe ausführlich F.M. Bakker et al. (1993), S. $1105 f$.
} 
empfunden. ${ }^{143}$ Vielmehr wird auch von wissenschaftlicher Seite die Befürchtung geäußert, daß die durch andere Risikomerkmale belasteten Versicherer versuchen würden, deren Träger im Sinne einer "adverse selection" aus ihrem Versichertenbestand fernzuhalten. Möglichkeiten dazu bestünden trotz Kontrahierungszwang etwa durch entsprechende Auswahl der Leistungspartner oder durch gezieltes Marketing. ${ }^{144}$ Der Wettbewerb würde somit in eine volkswirtschaftlich unsinnige und sozialpolitisch bedenkliche Richtung gelenkt.

Es gilt aber allenthalben als unsicher, ob eine möglichst weitgehende Verfeinerung der Kriterien zur Bemessung der Normzuwendungen überhaupt zum Erfolg führen kann. Eine mit dem Problem der Budegtierung der Versicherer befaßte Regierungskommission hat kürzlich dafür plädiert, die in ihren Augen fruchtlose und hinderliche Diskussion abzubrechen ${ }^{145}$ und als Verteilungsschlüssel allein den Anteil älterer Versicherter zu verwenden. Die Probleme sonstiger Risikogruppen seien besser im Wege eines verpflichtenden Pooling entsprechender Schadensfälle über alle Versicherungen $\mathrm{zu}$ lösen. ${ }^{146} \mathrm{Ob}$ dieser Weg tatsächlich weniger neue Probleme aufwirft $^{147}$, sei dahingestellt.

Der zweite wesentliche Grund dafür, daß eine echte Risikoübernahme durch die einzelnen Kassen einstweilen nicht durchsetzbar erscheint, liegt wiederum in deren geringen Handlungsspielräumen gegenüber den Leistungsanbietern (siehe a). Solange die Leistungspreise für die Versicherer ein Datum sind, können sie allenfalls im Verwaltungsbereich versuchen, Budgetüberschreitungen aufzufangen. ${ }^{148}$

\footnotetext{
${ }^{143}$ vgl. Ziekenfondsraad (1993), S. 43

${ }^{144}$ Siehe dazu ausführlich van der Ven/van Vliet (1992), S. 28ff. Zur Diskussion um die "Normzuwendungen" siehe auch R. van Duuren/B.H. Posthuma/F.A.M. Ruygt (1989).

${ }^{145}$ Toetsingscommissie Budgettering Verzekeraars (1993), S. 4f.

146 vgl. ebenda, S. 21

${ }^{147}$ Es wird nicht einfach sein, einen angemessenen Katalog solcher Extrarisiken zu vereinbaren und im Einzelfall auch zweifelsfrei anzuwenden. Sowohl ein unerwünschtes Zurückgleiten in eine faktische Einheitsversicherung als auch die Erschleichung von Wettbewerbsvorteilen durch einzelne Kassen können befürchtet werden. Zur Problematik von Pools siehe auch J. Wasem (1991), S. 388.

148 Dieser Punkt wird auch in Deutschland in Zusammenhang mit der hiesigen Gesundheitsreform diskutiert (Siehe etwa K. Jacobs (1993), S. 600f.).
} 
Ein Ergebnis der Halbherzigkeit, mit der die Erzeugung von Kassenwettbewerb bislang betrieben worden ist, ist die fast vollständige Gleichheit der "nominalen Prämien". ${ }^{149}$ Ebenso werden die gesetzlich inzwischen möglichen Policen mit Selbstbehalt bislang nicht angeboten, da der damit verbundene Prämienentgang für die Versicherer weitaus größer wäre als die Ersparnis, die ihnen nach dem zweistufigen Ausgleich effektiv verbleibt. Mit einer speziellen staatlichen Subvention soll der fehlende Anreiz nunmehr "künstlich" (und kostspielig) erzeugt werden. ${ }^{150}$

\section{ad c): Die Rolle des Staates}

Die bisherigen Darlegungen und der zuletzt genannte Vorgang zeigen bereits, daß auch der beabsichtigte Rückzug des Staates aus dem eigentlichen Marktgeschehen im Gesundheitswesen unter den derzeit gegebenen Umständen nicht gelingen kann. Die Wirren des Übergangs vom alten zum neuen System scheinen den Regulierungsbedarf mitunter sogar noch zu erhöhen.

Ein zusätzliches Problem wird dabei durch den Zielbegriff der "Kostenbeherrschung" markiert. Dieser ist nach Feststellung des Ziekenfondsraad bislang nicht klar definiert worden ${ }^{151}$, scheint aber das Bestreben auszudrücken, das Beitragsvolumen letztlich unter zentraler politischer Kontrolle zu halten. Die Praxis der Festlegung eines Makrobudgets ${ }^{152}$ entspricht dieser Vorstellung, steht aber im Gegensatz zu der Idee, die Verantwortung für die Entwicklung des Systems den Akteuren selbst zu übertragen. Es gibt keine Anzeichen dafür, daß eine Aufhebung der zentralen Kostendeckelung vorgesehen wäre. $\mathrm{Zu}$ fraglich ist offenbar der von den Neuregelungen erhoffte kostendämpfende Effekt. Zweifel daran werden zum einen mit dem eher geringen

\footnotetext{
${ }^{149}$ siehe Ziekenfondsraad (1993), S. $13 \mathrm{f}$.

${ }^{150}$ siehe F.M. Bakker et al. (1993)

${ }^{151}$ Ziekenfondsraad (1993), S. 83f.

152 Wie oben erwähnt, müssen Überschreitungen dieses Budgets in erster Instanz zwar hingenommen und mit Nachbewilligungen zum größten Teil alimentiert werden. Im jeweils folgenden Jahr werden sie aber durch Eingriffe in das Leistungswesen nach Möglichkeit wieder kassiert (Ziekenfondsraad (1993), S. 84).
} 
Umfang der "nominalen Prämie" begründet, die der Preiskonkurrenz zwischen den Kassen zu wenig Raum biete. ${ }^{153}$

Zum anderen wird mit der oligopolistischen Struktur der relevanten Märkte und mit den Präferenzen der Versicherten argumentiert, die erfahrungsgemäß für ein Höchstmaß an Schutz beinahe beliebig hohe Prämien in Kauf nähmen und wenig Experimentierfreude zeigten. ${ }^{154}$ Bislang jedenfalls sind kostendämpfende Wirkungen höchstens punktuell zu verzeichnen, was aber schon aufgrund der geringen Reformfortschritte nicht überraschen kann.

Zusammenfassend muß festgestellt werden, daß die an verschiedenen Punkten des Gefüges ansetzenden Veränderungen, die für ein Gelingen der Reform ineinandergreifen müßten, sich derzeit eher gegenseitig blockieren. Neben politischen Widerständen der zahlreichen betroffenen Gruppen und - in Zusammenhang damit - Unklarheiten in bezug auf die Ziele der Umgestaltung sind auch Steuerungsmängel feststellbar, die auf fehlende Einsicht in die relevanten Reaktionszusammenhänge zurückgehen. Insofern bleibt zu hoffen, daß der Reformverlauf zumindest einige Erfahrungswerte hervorbringt, die sich anderswo nutzen lassen.

\subsubsection{Auswirkungen im Bereich der Pflegeversicherung}

In bezug auf den Pflegesektor wird die für das Gesamtprojekt gültige Feststellung, daß allein "feststeht, daß nichts feststeht" ${ }^{155}$ noch verstärkt durch die Unsicherheit darüber, inwieweit gerade dieser Sektor von manchen der (eventuellen) neuen Regelungen ausgenommen bleibt. ${ }^{156}$ Der Ziekenfondsraad hat sich beispielsweise wiederholt dafür ausgesprochen, den "klassischen Care-Bereich" bei der Durchsetzung eines stärker marktorientierten Systems zumindest solange auszuklammern, bis der Fortbestand quantitativ ausreichender und qualitativ angemessener Versorgungsangebote durch geeignete flankierende Maßnahmen als gesichert gelten kann. ${ }^{157}$

\footnotetext{
${ }^{153}$ Vgl. J.G.A. van Mierlo (1991), S. 1165. Auch die Kommission zur Budgetierungsfrage plädiert daher für einen sehr kräftigen Ausbau dieser Prämienkomponente, der freilich starke Verwerfungen in der Nettoeinkommensverteilung zur Folge hätte (siehe Toetsingscommissie (1993), S. 29).

154 so C.A. de Kam (1990), S. $643 \mathrm{f}$.

15s van den Broek (1993), S. 951

156 siehe J.J. Poeisz/D. Veluwenkamp (1991), S. $10 \mathrm{f}$.

157 siehe Ziekenfondsraad (1991), S. 42f.
}

156 
Als Grund dafür wird dort angeführt, daß die Betroffenen "nur unzureichend in der Lage sind, ihre Interessen wahrzunehmen" und daher besonders geschützt werden müßten ${ }^{158}$, zumal sie für die Versicherer oftmals eine dauerhafte und große Belastung darstellten. Folglich könnten diese auf Strategien zur Ausgrenzung solcher Risiken sinnen. Diese Bedenken betreffen freilich nicht ausschließlich den Pflegefall und diesen nur insoweit, wie er beim einzelnen Versicherten im Voraus absehbar bzw. als wahrscheinlich erkennbar ist. Zumindest Alter und Geschlecht als wesentliche Merkmale des Pflegerisikos sollen im übrigen im Wege des Risikostrukturausgleichs "neutralisiert" werden, so daß idealerweise für die Versicherungen kein Anreiz besteht, etwa Frauen oder Ältere aufgrund ihres gesteigerten Pflegerisikos vom Beitritt abzuschrecken.

Aber auch wenn keine "adverse selection" stattfindet, besteht die Gefahr, daß Versicherungen Anbieter minderwertiger Pflege unter Vertrag nehmen, um Kosten zu sparen. Auch dies gilt freilich zunächst für alle Teilbereiche des Gesundheitswesens, so daß eine verstärkte staatliche Qualitätskontrolle ohnehin als notwendiges Element der Reform angesehen wird. ${ }^{159}$ In bezug auf die Pflegebedürftigen wird aber befürchtet, daß sie weniger als andere Konsumenten in der Lage seien, auch selbst über die Einhaltung von Leistungsstandard zu wachen und gegen deren mögliche Verletzung vorzugehen.

Ob derlei Befürchtungen dazu führen werden, Pflegeleistungen vom reformierten Finanzierungs- und Versorgungssystem auszunehmen, ist bislang nicht abschließend geklärt. So hat die Regierungskommission Bruins-Slot im Jahre 1993 in direktem Gegensatz zu den Bedenken des Ziekenfondsraad dafür plädiert, gerade den Pflegebereich zum Schauplatz eines Großversuchs zu machen, in dem die Neugestaltung der Beziehung zwischen Versicherern und Anbietern geprobt werden soll. ${ }^{160}$ Von den bislang ergriffenen Maßnahmen hinsichtlich der Budgetierung der Versicherer und der Schaffung von Vertragsfreiheit sind zumindest die Pflegeheime jedenfalls nicht betroffen. Bereits erfaßt ist hingegen auch die für die Pflege zuständige Volksversicherung von der genannten Spaltung des Beitrags durch Einführung einer pro

\footnotetext{
${ }^{158}$ siehe auch Eerste Kamer (1991), S. 7 (Abgeordnete Vriens-Auerbach) und S. 24 (Staatssekretär Simons)

${ }^{159}$ vgl. Ziekenfondsraad (1991)

${ }^{160}$ siehe Toetsingscommissie Budgettering Verzekeraars (1993), S. 28
} 
Versicherung konstanten Zusatzprämie neben dem einkommensabhängigen Beitrag. Die "Nominalprämie" wird seit 1992 erhoben und betrug 1993 im Durchschnitt 128 hfl pro Kopf und Jahr. ${ }^{161}$ Im Zuge der kürzlich erfolgten Abkehr vom ursprünglichen Umsetzungskonzept (siehe oben) soll sie aber nunmehr wieder abgeschafft werden. ${ }^{162}$

Es bleibt somit völlig offen, ob die in ihrer Durchsetzung insgesamt zweifelhafte Reorganisation des Gesundheitswesens auch im Bereich der Pflegedienste greifen und auf längere Sicht zu neuen und eventuell wirtschaftlicheren Leistungs- und/oder Kostenstrukturen führen wird. Etwas zögerlich beginnen die Anbieter sich vorsorglich auf veränderte Bedingungen einzustellen, die durch einen "Verlust an Sicherheit", aber auch durch erweiterte unternehmerische und pflegerische Möglichkeiten gekennzeichnet sein würden. ${ }^{163}$ Wann und wie diese Aussicht jedoch im Zusammenspiel gesetzlicher Veränderungen und tatsächlicher Reaktionen seitens der Versicherer (und ihrer Kunden) darauf konkrete Formen annehmen wird, ist auch für sie nicht absehbar. ${ }^{164}$

Eine nicht unwesentliche aktuelle Änderung im Bereich der Pflegeversicherung kann allerdings im Zusammenhang der generellen Reformbewegung gesehen werden. Es ist dies das nach einer Testphase nunmehr offenbar vor der flächendeckenden Einführung stehende Konzept des "klientenbezogenen Budgets" in der ambulanten Pflege. ${ }^{165}$ Es sieht vor, daß Hilfeempfänger zwischen dem üblichen Sachleistungsbezug und einem Geldbetrag wählen können, der in voller Höhen den Kosten der bewilligten Sachleistungen entspricht. Im Testgebiet haben knapp die Hälfte der Betroffenen von der neuen Möglichkeit gebraucht gemacht; die dabei gesammelten Erfahrungen werden als ermutigend bewertet.

\footnotetext{
${ }^{161}$ siehe J. Bruinsma (1993a); für 1994 wird dort mit rd. 140 hfl gerechnet, für Kinder gilt ein ermäßigter Satz.

${ }^{162}$ siehe Trouw vom 13.8.1994: Eigen risico voor iedereen

${ }^{163}$ Poeisz/Veluwenkamp (1991), S. 11

${ }^{164}$ vgl. ebenda, S. 21

${ }^{165}$ Zum folgenden siehe ausführlich T. Miltenburg et al. (1993).
} 


\subsection{2 "Substitution" als Antwort auf demographischen Druck}

Auch in den Niederlanden nehmen Anzahl und Anteil der älteren Menschen in der Gesamtbevölkerung in den nächsten Jahrzehnten deutlich zu (siehe Tabelle 5.4). Da dies nicht nur die über 65jährigen insgesamt betrifft, sondern zusätzlich innerhalb dieser Gruppe der Anteil der Hochbetagten steigt, ist von einer "doppelten Vergreisung" die Rede.

\section{Tabelle 5.4}

Alte Menschen in den Niederlanden - Prognose

\begin{tabular}{||c|c|c||}
\hline \multirow{2}{*}{ Jahr } & Anzahl in Tausend & Bevölkerungsanteil \\
\cline { 2 - 3 } & \multicolumn{2}{|c|}{ der über 65 jährigen } \\
\hline 1990 & 1.909 & $12,8 \%$ \\
\hline 2000 & 2.162 & $13,8 \%$ \\
\hline 2010 & 2.437 & $15,1 \%$ \\
\hline 2020 & 3.049 & $18,8 \%$ \\
\hline
\end{tabular}

Quelle: $\quad$ Tweede Kamer (1990), S. 13

Eine proportionale Ausweitung aller Pflegekapazitäten wäre absolut mit deutlichen Kostensteigerungen und - angesichts der Anteilsverschiebungen - mutmaßlich auch mit einem erhöhten Beitragssatz verbunden, da die Last zum größten Teil von der relativ schrumpfenden Bevölkerung im Erwerbsalter getragen werden muß. ${ }^{166}$ Schon bis zum Jahr 2000 würde der demographische Prozeß bei unveränderten Heimquoten einen Ausbau der stationären Kapazität um gut 20\% gegenüber 1987 auf mehr als 60.000 Plätze erfordern. ${ }^{167}$

\footnotetext{
${ }^{166}$ Zwingend ist das nicht, da eine erhöhte Erwerbsquote unter den Erwerbsfähigen und ein kräftiges Wachstum ihrer beitragspflichtigen Einkommen die Mehrbelastung kompensieren könnte. Zudem ist die Einkommensentwicklung bei den Älteren selbst zweifach von Belang, da sie sowohl auf das Beitragsaufkommen als auch auf den Umfang der Selbstbeteiligung einwirkt.
}

${ }^{167}$ A. Klaassen van den Berg Jeths (1991), S. 20 
Vor diesem Hintergrund stehen die seit einigen Jahren erkennbaren vielfältigen Bemühungen um ein verändertes Versorgungsprofil. Innerhalb des Pflegebereichs ${ }^{168}$ geht es in erster Linie darum, die stationäre Pflege zugunsten ambulanter Dienste in dem Maße zurückzudrängen, wie dies pflegerisch vertretbar und mit Kostenersparnis verbunden ist. Der Kommission zur Reform des Gesundheitswesens schwebt dabei eine Größenordnung von 25-35\% vor, um die die Anzahl der Pflegeplätze gegenüber der Fortschreibung des heutigen Gebrauchs langfristig gesenkt werden könne. ${ }^{169}$ Bis zum Jahr 2000 wird eine Kürzung um $20 \%$ für wünschenswert erachtet, was einem leichten absoluten Rückgang der Bettenzahl gegenüber 1987 gleichkäme. Im Gegenzug sieht dieses Szenario einen kräftigen Ausbau der ambulanten Dienste vor.

Bei diesen Werten handelt es sich eher um normative Vorgaben denn um geprüfte Optionen. Ob und wie der relative Rückbau der Heime unter Wahrung einer in allen Fällen ausreichenden Versorgung und angesichts von schon heute bestehenden Wartelisten tatsächlich vollzogen werden kann, ist unklar. Es mangelt an umfassenden Kenntnissen über Art und Stärke von Substituitionsbeziehungen zwischen den verschiedenen Pflegeformen. So haben sich zum Beispiel im Vergleich ausgesuchter Regionen mit unterschiedlichem Niveau ambulanter Versorgung in Hinblick auf die Nachfrage nach stationären Leistungen keine Unterschiede feststellen lassen. ${ }^{170}$ GOUDRIAAN berichtet von ähnlichen Resultaten verschiedener anderer empirischer Studien. ${ }^{171}$ Auch bei den verantwortlichen Politikern ist mittlerweile große Skepsis gegenüber einer raschen Realisierbarkeit der Umschichtungsprogramme eingekehrt. Der derzeit in Gegensatz zu den angesprochenen Visionen betriebene Ausbau des Pflegeheimsektors wird damit begründet, daß die stationäre Betreuung "ungeachtet aller Bemühungen, alte Menschen außerhalb von Einrichtungen zu unterstützen, für eine große Zahl dieser Älteren notwendig bleiben wird". ${ }^{172}$

\footnotetext{
${ }^{168}$ Ausgeklammert ist damit die ebenfalls erwünschte Verschiebung zwischen Krankenhäusern und Pflegeheimen zugunsten der letzteren (vgl. F.G.W. Goudriaan (1990), S. 21). Eine daraus resultierende Verteuerung der Pflegeversicherung würde durch Einsparungen bei der Krankenversicherung überkompensiert.

${ }^{169}$ vgl. F.G.W. Goudriaan (1990), S. 25

${ }^{170}$ siehe A.J. Stikker (1987), S. 62. Die Autorin vermutet, daß ihr Negativbefund auf die Schwierigkeit zurückgeht, anderweitige Einflußfaktoren zu eliminieren.

171 siehe Goudriaan (1990), S. $10 \mathrm{f}$.

${ }^{172}$ Tweede Kamer (1994), S. 91
} 
Andere Untersuchungen sprechen gleichwohl ebenso wie lokale Experimente dafür, daß ein beträchtliches Substitutionspotential vorhanden und realisierbar ist. Für die Provinz Zeeland wurde ermittelt, daß diejenigen Bewohner von Pflegeheimen zu geringeren Kosten auch daheim versorgt werden könnten, die weniger als drei Pflegestunden pro Tag beanspruchen. Dies sei bei etwa einem Drittel der Betroffenen der Fall. ${ }^{173} \mathrm{Zu}$ ähnlichen Resultaten gelangt der niederländische Rechnungshof, der eine kleine Gruppe von Personen untersucht hat, die nach erfolgter Indikation auf die Aufnahme in Pflegeheime warteten. In neun von 26 Fällen wäre eine - allerdings gegenüber der tatsächlich gewährten ausgeweitete - häusliche Betreuung als Alternative zur Einweisung in Betracht gekommen. ${ }^{174}$

Die praktische Umsetzung solcher Feststellungen ist modellhaft in einer niederländischen Kleinstadt unternommen worden. ${ }^{175}$ Durch konsequente organisatorische und finanzielle Zusammenführung aller Pflegeanbieter und eingehende Bedarfsanalyse in jedem einzelnen Fall ist es dort gelungen, die Heimpflegequote auf die Hälfte des Landesdurchschnitts zu senken. Die Kosten der verstärkten häuslichen Pflege betragen dort auch in schweren Fällen nur rd. ein Viertel der Heimpflegesätze. Auch wenn diese Resultate schon wegen der kleinen Fallzahl nicht als repräsentativ gelten können, so weisen sie doch die Richtung für Lösungswege, auf denen den Folgen der demographischen Veränderung ohne inakzeptable Kostensteigerungen oder Leistungseinschnitte begegnet werden kann. Gleichzeitig zeigen sie an, daß das herkömmliche System der Zuteilung von Pflegeleistungen Koordinationsmängel aufweist, auf die bei seiner Bewertung im folgenden Kapitel noch einzugehen ist.

Auch die Pflegeheime selbst bemühen sich, durch neue Angebote die als zu starr wahrgenommene Grenze zwischen häuslicher und stationärer Versorgung aufzulockern. Neben die schon etablierte Tagespflege treten dabei eine ganze Reihe von Teilzeitangeboten. Insbesondere die vorübergehende Aufnahme in Krisenfällen sowie zur Ermöglichung von Urlaub und Ruhepausen für die pflegenden Angehörigen sind auf rege Nachfrage gestoßen. ${ }^{176} \mathrm{Ob}$ solche Programme per saldo Kosten sparen, ist

\footnotetext{
${ }^{173}$ referiert bei Goudriaan (1990), S. 30

174 ebenda, S. 26

175 vgl. zum folgenden E.Hollander/J.H. Visser (1991)

${ }^{176}$ Siehe G. ten Haaft (1988). Als weitere Optionen werden dort die Intervall-Aufnahme (planmäßiger Wechsel zwischen Heim und häuslicher Umgebung im Jahresablauf), die Nachtaufnahme, die WochenendAufnahme und schließlich die regelmäßige Aufnahme für eine Reihe von Werktagen genannt.
} 
freilich nicht sicher, da die Entlastung auch von solchen Pflegefamilien in Anspruch genommen werden dürfte, die ohnehin keinen Übergang auf die vollstationäre Pflege in Erwägung ziehen. Nach dem Eindruck von Beteiligten ist aber von einem deutlichen Substitutionseffekt auszugehen. ${ }^{177}$

Abschließend sei zur Klarstellung in Hinblick auf die folgende Bewertung des Sicherungssystems angemerkt, daß die soeben skizzierten Substitutionsbemühungen weitgehend innerhalb des Spektrums der Versicherungsleistungen angesiedelt sind. Eine Auslagerung von Lasten in den Bereich der informell-privaten Pflege wird in großem Umfang nicht für realistisch bzw. vertretbar gehalten. Allenfalls könne es darum gehen, die Bedingungen für den Verbleib der Pflegebedürftigen im häuslichen Umfeld weiter zu verbessern, indem "professionelle Dienste als Ergänzung der informellen Pflege vorhanden sein müssen". ${ }^{178}$

Ebensowenig spielt in der Substitutionsdebatte die Vorstellung eine Rolle, wonach die mitunter unnötige Heimpflege als Folge versicherungsbedingter Fehlanreize anzusehen und durch deren Korrektur abzustellen sei. Diese Sicht wäre schon deswegen nicht plausibel, weil im Bereich der Altenwohnheime, für die ein Versicherungsschutz nicht besteht, noch weitaus größere Substitutionspotentiale vermutet werden. ${ }^{179} \mathrm{Zwar}$ wird in einer ebenfalls bei GOUDRIAAN referierten Untersuchung festgestellt, daß es bei niedrigen Einkommen "in einer Anzahl von Fällen billiger ist, intramural untergebracht zu sein, als selbständig wohnen zu bleiben". ${ }^{180}$ Dieser Befund betrifft aber Pflege- und Altenheime gleichermaßen und ist daher nicht der Pflegeversicherung, sondern der allgemeinen Mindestsicherung zuzuschreiben.

\footnotetext{
177 ebenda, S. 759

${ }^{178}$ Goudriaan (1990), S. $120 \mathrm{f}$.

${ }^{179}$ siehe A. Klaassen van den Berg Jeths (1991), S. 20

${ }^{180}$ Goudriaan (1990), S. 42
} 


\section{Zur Bewertung der gesetzlichen Pflegeversicherung in den Niederlanden}

Ziel des folgenden Kapitels ist es, zu einer Bewertung des niederländischen Konzepts der Pflegesicherung und deren bisheriger Funktionsgeschichte zu gelangen. Dies geschieht entlang der in den Kapiteln 3 und 4 aufgestellten Kriterien hinsichtlich der Zielwirkung und der Effizienz.

\subsection{Zur Bewertung des Systems unter Zielgesichtspunkten}

\subsubsection{Vorbemerkung in Hinblick auf erklärte Ziele}

Bevor die zu diesem Punkt aufgestellten allgemeinen Kriterien zur Anwendung kommen, erscheint es sinnvoll zu ermitteln, welche Absichten der niederländische Gesetzgeber selbst erklärtermaßen verfolgt hat. Einige Hinweise darauf sind bereits in der Darstellung zur Entstehungsgeschichte des betreffenden Gesetzes gegeben worden. Wie dort dient auch hier die ministerielle Begründung des Gesetzes als Schlüsseltext. Äußerungen der im Vorfeld zu Rate gezogenen Konsultativgremien werden in diesem Papier in Auszügen referiert.

Am Anfang steht die Einstufung der Pflegebedürftigkeit als allgemeines Lebensrisiko, dessen Absicherung weder auf privatem Wege zu bewerkstelligen noch im Leistungskatalog der bestehenden Sozialversicherung enthalten sei. Dieser Zustand wird als "unbefriedigend" bewertet, da er "nicht in Einklang mit den Grundsätzen gesellschaftlicher Solidarität und persönlicher Verantwortung " stehe. ${ }^{1}$ Abhilfe zu schaffen, sei daher "eine Sache von außerordentlich großer Dringlichkeit".

Auf Basis einer solchen Einschätzung des Ausgangszustands wird die Zielvorstellung der gesetzlichen Initiative umrissen. Dies geschieht teilweise nur implizit und an verschiedenen über den Text verstreuten Stellen. Folgende Leitmotive lassen sich dennoch identifizieren:

- $\quad$ ein quantitativer Ausbau des (stationären) Pflegesektors und die Anhebung seiner Versorgungsqualität auf ein angemessenes Niveau,

\footnotetext{
${ }^{1}$ G.M.J. Veldkamp et al. (1966), S. 8

${ }^{2}$ ebenda, S. 2
} 
- dabei insbesondere eine konsequente Verstärkung der Bemühungen um Rehabilitation, selbst wenn die dabei erzielbaren Erfolge vielfach "nur von geringem Umfang sein werden", ${ }^{3}$

- die Ablösung der bisher dominanten Finanzierung über die Mindestsicherung im Sinne eines Übergangs "von der Sphäre der Fürsorge in die Rechtssphäre", 4

- die Entlastung überforderter Pflegefamilien und

- $\quad$ die Schaffung einer dauerhaft verläßlichen Finanzierungsgrundlage.

Zum letzten Punkt wird der "Sozial-ökonomische Rat" mit der Einschätzung zitiert, daß bei Finanzierung aus Steuergeldern "die verfügbaren Mittel dadurch von der Haushaltspolitik des Staates abhängig werden könnten, was der Rat für unerwünscht hielte". 5 $\mathrm{Da}$ die zu schaffende Versicherung abgesehen vom erwähnten "Nachholbedarf" auch aufgrund der zunehmenden Zahl alter Menschen ein mutmaßlich steigendes Leistungsvolumen zu bewältigen haben wird, ist den Autoren der Gesetzesvorlage bewußt. ${ }^{6}$

Die Liste der Zielvorstellungen ist mit dem im Kap.3 erstellten Katalog weitgehend kompatibel. Zudem spielt auch die dort gesondert angeführte Vorstellung ${ }^{7}$ eine Rolle, wonach die Versorgung mit Pflegeleistungen aufgrund ihrer existenziellen Qualität allein nach Bedarfsgesichtspunkten und ohne Ansehen der individuellen Zahlungsfähigkeit zu erfolgen habe. Eine solche Forderung wird zwar nicht ausdrücklich erhoben, ist aber impliziert, wenn es heißt, daß auf Basis des Pflegegesetzes "jeder Niederländer, den ein solch schweres Schicksal trifft, Anspruch auf eine optimale sozialmedizinische Versorgung geltend machen kann, optimal in Bezug auf den Stand von Wissenschaft und Technik". ${ }^{8}$ Einer solchen Intention entspricht denn auch das gewählte Sachleistungskonzept, ohne daß allerdings ein solcher Zusammenhang im Text hergestellt würde. Für diese Entscheidung bezüglich der Leistungsform fehlt überhaupt jede Begründung; monetäre Transfers scheinen als Alternative während der Vorbereitung des Gesetzes nicht in Betracht gezogen worden zu sein.

\footnotetext{
${ }^{3}$ Veldkamp et al. (1966), S. 16

${ }^{4}$ ebenda, S. 2

5 ebenda, S. 6

${ }^{6}$ ebenda, S. 18

${ }^{7}$ siehe Abschnitt 3.3

${ }^{8}$ Veldkamp et al. (1966), S. 16, Hervorhebung hinzugefügt
} 
Auf dem Hintergrund dieser skizzenhaft geordneten Wiedergabe erklärter Ziele können nunmehr die einzelnen Punkte des Kriterienkatalogs in Hinblick auf die Effektivität der Absicherung abgearbeitet werden.

\subsubsection{Reichweite}

Dieser Aspekt läßt sich im Grundsatz rasch erledigen, da die Pflegeversicherung wie erwähnt laut Gesetzestext alle Personen umfaßt, die in den Niederlanden wohnen oder der niederländischen Einkommensteuer unterliegen. ${ }^{9}$ Diese Bestimmung sieht mithin weder eine Wartezeit ${ }^{10}$ noch die Entrichtung eigener Beiträge als Leistungsbedingung vor. Offensichtlich ist sie vorrangig darauf ausgerichtet, personale Sicherungslücken restlos zu vermeiden. Als Kriterium für die juristische Festlegung des Wohnortes dient der "Mittelpunkt des gesellschaftlichen Lebens", zu dessen Operationalisierung im Einzelfall wiederum eine Vielzahl von Merkmalen herangezogen werden kann, die in ihrer Gesamtheit zu werten sind. So wird etwa bei Ausländern, die Ansprüche geltend machen wollen, das Vorliegen einer Aufenthaltsgenehmigung weder als hinreichende, noch als notwendige Bedingung der Leistungsgewährung angesehen. ${ }^{11}$ Weitere Einzelheiten zur Auslegung der Abgrenzungsvorschrift brauchen hier nicht verfolgt $\mathrm{zu}$ werden.

Dem Kriterium der Vollständigkeit in Hinblick auf Kreis der Leistungsberechtigten genügt die in den Niederlanden vorfindliche Absicherung also ohne Abstriche. Die Bedingungslosigkeit des Anspruchs ist relevant und sachgerecht insbesondere in Hinblick auf (hier ansonsten nicht betrachtete) Fälle angeborener Pflegebedürftigkeit. ${ }^{12}$

\subsubsection{Leistungszugang}

Die tautologische Definition des Bedarfsfalls für die stationäre Pflege sowie ihre herrschende Auslegung sind bereits dargestellt worden. Im Zuge der Reform des niederländischen Gesundheitssystems haben die Akteure viel Wert darauf gelegt, derartige, auf Institutionen (hier: das Pflegeheim) bezogene Umschreibungen des Leistungs-

\footnotetext{
${ }^{9}$ siehe Art. 5.1 und Art. 2

${ }^{10} \mathrm{vgl}$. Informatiecentrum Zorg en Welzijn (IZW) (1992), S. 1

${ }^{11}$ ebenda, S. 2

${ }^{12}$ vgl. dazu R. Thiede (1990), S. 114
} 
anspruchs durch funktionale Bestimmungen zu ersetzen ${ }^{13}$, um die Flexibilität des Leistungswesens zu erhöhen. Für den als "Pflege und Versorgung" abgegrenzten Funktionsbereich sollte eine Anspruchsdefinition neuen Typs nach dem ursprünglichen Zeitplan bereits Anfang 1992 in Kraft treten. ${ }^{14}$ Dieser Termin ist jedoch dreimal verschoben worden; vor 1996 ist mit der anvisierten Novelliereung offenbar nicht mehr zu rechnen. ${ }^{15}$

Entscheidender als die Details der formalen Anspruchsdefinition ist aber das Verfahren ihrer Einlösung: die Indikation sowie deren Verbindung zum tatsächlichen Leistungsbezug. Es ist bereits angedeutet worden, daß die niederländische Pflegeversicherung in diesem Bereich erhebliche Probleme aufwirft. Ihnen soll hier nachgegangen werden.

\section{a) stationärer Sektor}

Vor Einführung des neuen Indikationsgremiums oblag die Entscheidung darüber, ob ein Bedarf im Sinne der obigen Definition vorliegt, im wesentlichen dem Hausarz $\mathrm{t}^{16}$ oder - je nach Art des Falles und nach regionalen Gepflogenheiten - verschiedenen Betreuungsinstanzen. ${ }^{17} \mathrm{Da}$ ihnen im allgemeinen kein Interesse an einer restriktiven Handhabung solcher Definitionsmacht unterstellt werden kann, ist zu vermuten, daß entsprechende Indikationen im Interesse der bedürftigen Patienten eher großzügig erteilt worden sind. Die Anzahl der Pflegeplätze hingegen ist aus Gründen der Kostenbegrenzung zunehmend knapp gehalten worden. Wie gezeigt, werden die hergebrachten Kapazitätsnormen, an denen sich die Lizensierungspraxis der Behörden orientiert, seit den 80er Jahren zunehmend unterschritten. Dadurch aber ist es für die Betroffenen schwieriger geworden, auf Basis ihres verbrieften Anspruchs auch tatsächlich einen Heimplatz zu erhalten.

In der Einteilung des niederländischen Heimwesens in somatische und psychogeriatrische Einrichtungen bzw. Pflegeplätze ist ein solcher Engpass vorrangig für die zuletzt genannte Fallkategorie feststellbar, deren Umfang auf der Bedarfsseite kon-

\footnotetext{
${ }^{13}$ vgl. etwa Nationale Raad voor de Volksgezondheid (1988), S. 4

${ }^{14}$ P.A.M. van den Broek (1993), S. 878

${ }^{15}$ ebenda, S. 950

${ }^{16} \mathrm{vgl}$. M. Kastelein et al. (1985), S. 32

${ }^{17}$ siehe P.J.N. Laurs/G.B. Teuben (1990), S. 1799 
tinuierlich zunimmt. In einer Erhebung für das Jahr 1987 werden dazu folgende Befunde ausgewiesen:

Für den somatischen Bereich betrug die mittlere Wartezeit zwischen Indikation und Aufnahme in ein Pflegeheim knapp sieben Wochen ${ }^{18}$; ein Wert, der sich vermutlich im Rahmen des friktionell Notwendigen bewegt, wenn ein hoher Auslastungsgrad der Einrichtungen gewährleistet werden soll. Psycho-geriatrische Patienten hingegen warten im Durchschnitt fast 25 Wochen, in einzelnen Provinzen sogar über 8 Monate lang auf einen freiwerdenden Platz. ${ }^{19}$ Unter Berücksichtigung der Tatsache, daß von den stationär untergebrachten Patienten dieser Kategorie jährlich mehr als ein Viertel stirbt $^{20}$, kann angenommen werden, daß eine beträchtliche Zahl von Aufnahmesuchenden das Ende der Wartezeit nicht mehr erlebt.

In einer Regionalstudie für den Raum Den $\mathrm{Haag}^{21}$ findet sich diese Annahme bestätigt: Es sind dort 1988 etwa 13\% der auf einen Platz im psycho-geriatrischen Pflegeheim Wartenden gestorben. Parallel zur Länge der Warteliste zeigt dieser Wert eine steigende Tendenz. In der hier untersuchten Provinz erfolgte die Verteilung der Plätze unter den Wartenden nicht durch das einzelne Heim, sondern über ein "Vermittlungbüro", das in besonders dringenden Fällen eine Plazierung an der chronologischen Warteliste vorbei vornimmt. Da auf diesem Wege zum Untersuchungszeitpunkt bereits mehr als ein Viertel der Plätze vergeben wurde, verlängert sich die Wartezeit in Fällen minderer Dringlichkeit. Im Durchschnitt wird sie für die Haager Psychogeriatrie mit 10,5 Monaten angegeben.

In anderen Regionen, wo ein derartiges koordiniertes Verfahren nicht praktiziert wurde, sind die Folgen der relativen Knappheit an Unterbringungsmöglichkeiten dadurch verschärft worden, daß die Vergabe der Plätze durch die einzelnen Heime eher zufällig und spontan als nach Dringlichkeit des Bedarfs erfolgte. Auch hat offenbar das Eigeninteresse der Einrichtungen mitunter dazu geführt, daß Personen mit ausgeprägt hohem

\footnotetext{
18 vgl. J.M. Spaan (1989), S. 40

19 Ebenda. Irritierenderweise wird dort auf die Möglichkeit hingewiesen, daß die Werte dadurch überzeichnet sein könnten, daß Patienten gleichzeitig auf mehreren Wartelisten plaziert sind. Wenn es sich um tatsächlich gemessene Wartezeiten handelt, dürfte dies keinen Einfluß haben.

${ }^{20}$ Errechnet aus Angaben ebenda, S. 53 und 14.

${ }^{21}$ siehe - für den gesamten Absatz - W.P. Elberse (1989), S. $42 f$.
} 
Pflegebedarf gerade nicht aufgenommen worden sind. ${ }^{22}$ Überdies schien "im Fall langer Wartezeiten (..) praktisch nie eine systematische Unterstützung oder Begleitung der betroffenen Patienten stattzufinden". ${ }^{23}$

Ein auch aus ökonomischer Sicht beachtliche Folgeerscheinung des Kapazitätsengpasses ist die Fehlbelegung von Krankenhausbetten durch Patienten mit Anspruch auf Pflegeplatz. 1990 wurden über 3300 offiziell deklarierte Fälle dieser Art gezählt, gegenüber 1988 eine Steigerung um über $50 \%{ }^{24}$

Entscheidend für die Frage, ob sich die Problematik der Wartelisten in jüngster Zeit und in naher Zukunft gegenüber den aufgeführten, nicht mehr ganz aktuellen Befunden verschärft oder abschwächt, sind zwei Entwicklungen: Auf der Angebotsseite die gegenwärtig verfolgte Kapazitätspolitik, hinsichtlich der wirksamen Nachfrage die Praxis des Bedarfsfeststellungsverfahrens. Zunächst sei der letztgenannte Faktor erörtert.

Inwieweit das bereits dargestellte neue und einheitliche, seit 1989 angewandte Indikationsverfahren die Aussicht der Betroffenen auf Einlösung ihres Pflegeanspruchs verbessern hilft, kann bislang noch nicht auf empirischen Wege beantwortet werden. Eine genauere Analyse des damit verfolgten Ansatzes zeigt aber, daß allenfalls eine bessere Abfederung, nicht jedoch eine Behebung der geschilderten Mängel erwartet werden kann.

Zunächst läßt sich feststellen, daß das Indikationsgremium offenbar nicht die Aufgabe hat, Angebotslücken oder auch -überhänge deutlich werden zu lassen und damit die Grundlage für eine ggfs. erforderliche Anpassung der Kapazitäten zu schaffen. Umgekehrt ist sie ausdrücklich gehalten, bei ihren Entscheidungen "den örtlich vorhandenen Einrichtungen Rechnung zu tragen". ${ }^{25}$ Diese Orientierung am Angebot ist zwar in pragmatischer Sicht sinnvoll, letztlich aber nicht vereinbar mit der Kernaufgabe,

\footnotetext{
${ }^{22}$ Siehe dazu J.B.V. Welten/C.A. ten Dam-van Lieshout (1987), S. 243: Für die Stadt Den Haag wird hier festgestellt, daß in schwereren Fällen längere Wartezeiten auftraten. " $\mathrm{Da} ß$ eher 'leichtere' Patienten aufgenommen wurden, kann von dem Wunsch herrühren, der Bewohnerschaft ein gemischtes Profil zu verleihen, oder auch aus dem Anliegen, das Pflegepersonal nicht zu stark zu belasten."

${ }^{23}$ M. Kastelein et al. (1985), S. 38

${ }^{24}$ Tweede Kamer (1992), S. 129

${ }^{25}$ Laurs/Teuben (1990), S. 1802
} 
die Bedürfnisse des Antragstellers unvoreingenommen zu bewerten. Eine sachfremde Relativierung der Leistungsansprüche ist nicht ausgeschlossen.

Im - derzeit aufgrund der zunehmend sparsamen Kapazitätssteuerung eher unrealistischen - Fall eines Überangebots an stationären Pflegeplätzen verschärft sich die Problematik dadurch, daß auch Vertreter der Einrichtungen selbst dem Gremium angehören dürfen. ${ }^{26}$ Es besteht dann die Gefahr, daß die vom Gesetzgeber ausdrücklich gewünschte Einbeziehung ambulanter Alternativen in den Entscheidungsprozeß faktisch zu kurz kommt.

Ob sich solche Befürchtungen bewahrheiten, hängt ebenso wie die Wirksamkeit der Kommissionen im angestrebten Sinn insgesamt von deren genauer Zusammensetzung $a b$, die der Gesetzgeber weitgehend offengelassen hat. Da auch die Verfahrensweise im einzelnen nicht standardisiert und die Berufungsmöglichkeit gegen einen abschlägigen Bescheid demzufolge faktisch beschränkt ist, wird eine "schlechte" (interessengeleitete, unzureichend kompetente, uninformierte, überlastete) Kommission auch schlecht funktionieren, ohne daß dem Einhalt geboten werden kann. Sind hingegen die personellen und finanziellen ${ }^{27}$ Voraussetzungen gegeben, kann der koordinierte und im Prinzip das gesamte Hilfsangebot umfassende Ansatz, der eine gewisse Verantwortung für die Bedürftigen und die Folgen der Indikationsentscheidung impliziert ${ }^{28}$, immerhin dazu führen, daß die jeweils gegebene Angebotsknappheit nach Bedarfsgesichtspunkten optimal verwaltet wird.

Kritisch anzumerken ist allerdings, daß die Kommission dabei auf die Mitwirkung der Pflegeheime selbst angewiesen ist. Deren Eigenmacht in Hinblick auf die Aufnahmeentscheidung bleibt nämlich auch im Rahmen des neuen Verfahrens unangetastet. ${ }^{29}$ Zwar soll die Kommission den Fortgang des Verfahrens nach Erteilung einer Indikation verfolgen und deren Umsetzung im Benehmen mit den Heimverwaltungen fördern; erwirken kann sie eine Aufnahme aber letztlich nicht. Dies in Verbindung mit dem nach ihrer Einschätzung bislang unzureichenden Leistungsangebot führt LAURS/-

\footnotetext{
${ }^{26}$ Sie verlieren allerdings ihr Stimmrecht in den Fällen, wo die eigene Einrichtung direkt betroffen ist (vgl. Vereniging van Nederlandse Gemeenten (VNG) (1988), S. 14). Ob diese Bestimmung ausreicht, um einen Einfluß von Eigeninteressen auszuschließen, erscheint zweifelhaft.

${ }^{27}$ Laurs/Teuben (1990), S. 1803, halten die für die Kommissionen bereitgestellten Mittel für gering.

${ }^{28}$ siehe im einzelnen VNG (1988), S. 14 und 19

${ }^{29}$ vgl. ebenda, S. 16
} 
TEUBEN zu dem harten Urteil, mit dem neuen Verfahren würden die Versorgungsprobleme lediglich "kaschiert und institutionalisiert". Das mit der Versicherung intendierte Recht auf Pflege bleibe "was es schon seit vielen Jahren ist, nämlich das Recht, auf eine Warteliste gesetzt zu werden" ${ }^{30}$.

Eine entspannende Wirkung scheint aber neuerdings von der Kapazitätspolitik auszugehen, die jüngste Trendwende in Richtung auf eine Hebung des Versorgungsgrades ist im Abschnitt 5.4 beschrieben worden. Auch wenn die Erweiterungsquote sich in ähnlicher Größenordnung bewegt wie das Ausmaß der in den 80er Jahren feststellbaren Wartelisten, wäre es angesichts der fortgesetzt wachsenden Nachfragebasis vorschnell, darin schon die völlige Aufhebung des Kapazitätsmangels zu erblicken. Gleichwohl ist das Ausbauprogramm in diesem Sinn ein bedeutender Schritt.

b) ambulanter Sektor

Die in diesem Bereich für das Ausmaß der gebotenen Leistungen relevanten Regelungen unterscheiden sich in einigen wesentlichen Punkten von denen des stationären Sektors, im Effekt aber zeigen sich hier ganz ähnliche Mängel und Reibungen. Zu unterscheiden ist innerhalb des Sektors stets zwischen den pflegerischen Diensten der "Kruiswerk"Vereine und der sogenannten "Familienhilfe", die primär auf Hilfestellung bei der Haushaltsführung ausgerichtet ist. Zunächst besteht eine allgemeine Beschränkung des Anspruchs auf die erstgenannte Hauspflege darin, daß regulär nicht mehr als drei Einsätze täglich mit einer Gesamtdauer von höchstens zweieinhalb Stunden gewährt werden. ${ }^{31}$ Es ist allerdings darüber hinaus eine ständige Rufbereitschaft geschaffen worden, auf die in Notfällen zugegriffen werden kann. ${ }^{32}$ Bei regelmäßig größerem Hilfsbedarf sind Betroffene gleichwohl auf Unterstützung durch Dritte bzw. den Zukauf von Leistungen privater Anbieter angewiesen, wenn eine Heimeinweisung vermieden werden soll.

Im übrigen sind auch im ambulanten Bereich die Indikationswege und deren Überlagerung durch Kapazitätsknappheiten entscheidend für die Möglichkeit, Hilfe in An-

\footnotetext{
${ }^{30}$ Laurs/Teuben (1990), S. 1803

${ }^{31}$ Nationale Kruisvereniging (1989), S. 35. Bei den in LVT (1990), S. 6 angegebenen dreieinhalb Stunden Höchstdauer handelt es sich offenbar um einen Irrtum.
}

${ }^{32}$ vgl. ebenda

170 
spruch zu nehmen. Im pflegerischen Zweig der ambulanten Dienste hat eine eigenständige Indikation traditionell nicht stattgefunden. Das Vergabeverfahren war in hohem $\mathrm{Maße}$ auf die jeweils zuständige Bezirkspflegerin konzentriert, die nach eigenem Gutdünken die Verteilung der jeweils vorhandenen Kapazitäten vornahm. ${ }^{33}$ Im Zuge der Zusammenführung des Kruiswerk mit der Familienhilfe wird derzeit ein formalisiertes Verfahren entwickelt, das für den gesamten ambulanten Bereich gültig sein soll. Auch wenn diese Regelung im einzelnen nach dem Stand der mir zugänglichen Papiere noch strittig ist ${ }^{34}$, zeichnet sich $a b$, daß sie weitgehend an das in der Familienhilfe bereits seit längerem praktizierte Konzept angelehnt sein wird. Hier entscheidet über das Vorliegen eines Bedarfs eine spezielle Instanz, die allerdings nicht wirklich unabhängig, sondern unter dem Dach der Leistungsanbieter angesiedelt ist.

Ihre Aufgabe ist die Ausfüllung der generellen Bestimmung, daß weder mehr noch weniger als "die notwendige Pflege, Versorgung etc. "35 bereitgestellt werden soll. Die Interpretation dieser Klausel ist gekennzeichnet vom Gedanken der Subsidiarität: "Das Unvermögen (...) des Nachfragers und seiner Hausgenossen, die ausgefallenen Funktionen (...) aus eigener Kraft angemessen zu regeln, markiert den Ausgangspunkt der Indikationsstellung. ${ }^{136}$ Es soll daher sorgfältig geprüft werden, inwieweit durch Rückgriff auf Ressourcen in der "Umgebung" des Klienten eine professionelle Versorgung überflüssig gemacht werden kann. Die Umgebung ist dabei allerdings wörtlich zu nehmen und umfaßt im wesentlichen die tatsächlich im Haushalt Anwesenden; Verwandte außer Haus werden also nicht in die Pflicht genommen. Wie die bei Anwendung dieses Ansatzes erforderlichen Zumutbarkeitsgrenzen gezogen werden, ist nicht ersichtlich und letztlich wohl eine Ermessensfrage. Betont wird jedoch, daß diesbezüglich ein Unterschied zwischen einem rein hauswirtschaftlichen Bedarf und echten Pflegeaufgaben gemacht wird. Von letzteren wird generell angenommen, daß sie privat schwerer zu bewältigen sind. ${ }^{37}$

\footnotetext{
${ }^{33}$ vgl. LVT (1990a), S. 5

${ }^{34}$ LVT (1990a) enthält einen Entwurf, der aber vom Pflegezweig der neuen Dachorganisation nicht akzeptiert worden ist (siehe LVT (1991), S. 13).

${ }^{35}$ LVT (1990a), S. 6, Hervorhebung im Original

${ }^{36}$ ebenda, S. 12, Hervorhebung hinzugefügt

${ }^{37} \mathrm{vgl}$. Stichting Centrale Raad voor Gezinsverzorging (CRG)/ Nationale Kruisvereniging (NKV)(1990), S. 14
} 
Wiederum ist die Anerkennung des Bedarfs nicht gleichzusetzen mit seiner Befriedigung. Vielmehr ist es möglich, "daß wegen Mangels an Kapazitäten die Hilfe, die dem Klienten zuteil wird, nicht vollständig oder nicht unverzüglich mit der notwendigen Versorgung übereinstimmt, wie sie mithilfe des Indikationsverfahrens festgestellt worden ist". ${ }^{38}$ Es wird daher wie im stationären Sektor für nötig gehalten, die Indikation um einen Dringlichkeitsvermerk zu ergänzen.

Es zeigt sich darin das schon bekannte Dilemma: Soll einerseits die Indikation erklärtermaßen allein nach objektiven und gleichbleibenden Bedarfsmaßstäben erfolgen $^{39}$, so wird andererseits davon unabhängig das verteilbare Volumen der Hauspflege jährlich von der Regierung festgesetzt. ${ }^{40}$ Die Kapazitätssteuerung erfolgt anders als im stationären Bereich nicht direkt mengenmäßig, sondern im Wege der Zuweisung von Mitteln, deren Verwendung den Organisationen der Leistungsanbieter anheimgestellt ist.

Wie groß die Kluft zwischen Bedarf und Deckung im Bereich der ambulanten Dienste ist, läßt sich nicht leicht feststellen bzw. bewerten. Ein Teil der Schwierigkeit resultiert aus dem problematischen Tatbestand, daß die Indikationen von den Anbietern selbst erteilt werden. Sie erstreben als gemeinnützige Körperschaften zwar keinen Gewinn, könnten aber dennoch geneigt sein, den Bedarf großzügig abzugrenzen, um damit Druck in Richtung auf Ausweitung ihrer Haushaltsmittel zu erzeugen. Belegen läßt sich gleichwohl, daß das Versorgungsniveau im Sinken begriffen ist: Die ambulanten Hilfsdienste sehen sich mit einer steigenden Zahl schwerer Fälle konfrontiert, die einem immer größeren Anteil der Kapazität binden und damit die Reichweite des Systems verringern.

So hat im pflegerischen Zweig die Anzahl der Besuche pro Klient und Jahr allein von 1985 bis 1990 um gut $68 \%$ zugenommen. Entsprechend ist der Bevölkerungsanteil, der die Dienste im Laufe eines Jahres überhaupt in Anspruch nimmt, im gleichen Zeitraum um mehr als ein Drittel gesunken. ${ }^{41}$ Bis 1992 verringerte er sich nochmals

\footnotetext{
${ }^{38}$ LVT (1990a), S. 9

${ }^{39}$ siehe Stichting CRG/NKV (1990), S. 15

${ }^{40}$ vgl. etwa o.V. (1992), S. 28

${ }^{41}$ errechnet aus NKV (1989), S. 14 und Tweede Kamer (1992), S. 151
} 
um $12 \% .^{42}$ Diese Entwicklung ist schon deswegen plausibel, weil die auf einen Heimplatz Wartenden in großem Umfang ersatzweise von ambulanten Hilfen Gebrauch machen dürften. Offensichtlich werden dadurch und wohl auch durch einen Trend zur ambulanten Pflege auf seiten der Nachfrager leichtere Fälle trotz anerkanntem Bedarf aus der ambulanten Pflege herausgedrängt.

In bezug auf die wachsenden alten Bevölkerungsgruppen ist die sinkende Reichweite der ambulanten Pflege - bei allerdings hohem Ausgangsniveau - ebenfalls deutlich erkennbar. Bei den 70-79jährigen nahmen 1992 noch 15\% (nach 22\% im Jahre 1985) solche Hilfen wenigstens einmal in Anspruch; unter den noch Älteren ist der entsprechende Anteil sogar von 56 auf $35 \%$ gefallen. ${ }^{43}$

Bei den Hilfen im Haushalt stellt sich die Situation umgekehrt dar: Eine deutliche Erhöhung der Klientenzahl wird hier mit einer sinkenden Stundenzahl pro Fall und Woche erkauft. ${ }^{44} \mathrm{Da}$ in diesem Segment bei Bedarf auch einfachere Pflegetätigkeiten enthalten sind, besteht offenbar eine gewisse Substitutionsbeziehung zwischen beiden ambulanten Zweigen: So wie die häusliche Pflege i.e.S. einen Teil der schwer Pflegebedürftigen mit Anspruch auf Heimplatz ersatzweise auffängt, landen dort verdrängte minder schwere Fälle zunehmend bei der "Familienhilfe".

Insgesamt hat sich die Knappheitslage in den letzten Jahren nach Darstellung von Beschäftigtenverbänden zusehends verschärft. In der Presse lassen sie verlauten, daß schon vor dem Wirksamwerden einer jüngst beschlossenen Mittelkürzung inzwischen $20 \%$ der induzierten Bedarfsträger zunächst ohne Hilfe bleiben. Die Wartezeiten beliefen sich mitunter auf "bis zu eineinhalb oder gar zwei Jahre". ${ }^{45}$ Um Abhilfe zu schaffen, sei ein Ausweitung des Mittelvolumens für den ambulanten Sektor insgesamt um mindestens ein Siebtel vonnöten

\footnotetext{
${ }^{42}$ Tweede Kamer (1994), S. 108

${ }^{43}$ NKV (1989), S. 14 und Tweede Kamer (1994), S. 108

44 siehe Tweede Kamer (1994), S. 107: Der Anstieg der Klientenzahl zwischen 1989 und 1992 beträgt bei den über 65jährigen knapp ein Viertel. Die durchschnittlich gewährte Stundenzahl pro Woche ist von 5,1 (1989) auf 4,3 (1993) gesunken.
}

${ }^{45}$ S. Baart (1993) 


\section{c) Schluß}

Für beide betrachteten Versorgungsebenen muß mithin festgestellt werden, daß das für die Versicherung konstitutive Leistungsversprechen nicht immer und vollständig eingelöst wird. Mag auch die Definition der Bedarfsfälle eine letztlich nicht allgemein faßbare und objektiv bewertbare Angelegenheit sein, so tritt doch die Diskrepanz zwischen Anspruch und Wirklichkeit durch die Trennung von Indikation und Leistungsgewährung recht offen zutage. War es zunächst offenbar nicht nur vom Bedarf, sondern auch vom Zufall abhängig, ob oder wann im Einzelfall tatsächlich Leistungen erbracht wurden, so laufen die jüngst vollzogenen oder eingeleiteten Verfahrensänderungen darauf hinaus, daß sich die Schwelle zum faktischen Leistungszugang allgemein erhöht: Nur in schwereren bzw. dringenden Fällen bleibt der Anspruch auf ambulante Hilfen zuverlässig gewahrt, nur bei sehr stark ausgeprägter Bedürftigkeit ist mit stationärer Pflege zu rechnen.

Ihren Grund hat diese Leistungsbeschränkung in der Deckelung des Gesamtvolumens durch die Regierung. Diese kontrolliert im Kontext ihrer Haushalts- und Wirtschaftspolitik das Beitragsvolumen und betreibt damit letztlich das, was in Zusammenhang mit der deutschen Pflegeversicherung als "einnahmeorientierte Ausgabenpolitik" bezeichnet worden ist. ${ }^{46}$ Für die politische Praxis ist indes anzunehmen, daß es nicht uneingeschränkt möglich ist, begründete Forderungen der Versicherten bzw. der Leistungsanbieter zu ignorieren. Die jüngsten Bemühungen um eine Entschärfung der Mangellage belegen dies. Radikale Einschnitte sind im übrigen auch deswegen nicht zu erwarten, weil kein direkter Zugriff auf Beitragsmittel zugunsten anderer Ausgabenzwecke besteht und weil ein dann drohender Zusammenbruch der Versicherung wiederum negative Folgen auch für den Staatshaushalt hätte. Ein gewisser Spielraum zur Beschneidung von Leistungsansprüchen auf Kosten des Versicherungsziels ist aber offenbar gegeben.

\subsubsection{Selbstbeteiligung}

Beschränkt ist der von der Pflegeversicherung gebotene Leistungsumfang durch die Selbstbeteiligung der Leistungsempfänger. Der absoluten Höhe nach relevant ist sie insbesondere im stationären Bereich. Der hier angewandte einkommensabhängige Tarif

\footnotetext{
${ }^{46}$ siehe etwa Presse- und Informationsamt der Bundesregierung (1994), S. 3
} 
ist bereits dargestellt und erläutert worden. ${ }^{47}$ Seine Anwendung läuft nach derzeitigem Stand darauf hinaus, daß

- $\quad$ das Einkommen fast vollständig abgeschöpft wird, soweit es einen Freibetrag weit unterhalb der Grundrente übersteigt und solange die Obergrenze des Selbstbehalts nicht erreicht ist,

- $\quad$ auch wohlhabende Pflegebedürftige weniger als die Hälfte der heute üblichen Pflegesätze selbst tragen,

- $\quad$ in keinem Fall die Mindestsicherung eintreten muß,

- $\quad$ das Vermögen der Leistungsempfänger unangetastet bleibt und daß

- diejenigen stationär Gepflegten weitgehend geschont werden, die einen Partner im vormals gemeinsamen Haushalt zurücklassen.

Im wesentlichen sind damit die zu diesem Punkt formulierten Kriterien in den Niederlanden erfüllt. Zumindest ist gewährleistet, daß jeder stationär Gepflegte in substantiellem Umfang von der Versicherung profitiert und daß eine durch die Pflegebedürftigkeit induzierte Armut nicht eintritt. Problematisch ist hingegen die Tatsache, daß ein Einkommensschutz faktisch nicht gewährt wird. Zwar enthält die Selbstbeteiligung auch das als unbedenklich und wünschenswert eingestufte Moment der Haushaltsersparnis, die bei Umzug in ein Pflegeheim realisiert wird ("unechte Selbstbeteiligung") und ist damit auch ursprünglich begründet worden. Bei einem Höchstsatz von $2.200 \mathrm{hfl}$ kann aber ohne weiteres davon ausgegangen werden, daß die Ersparnis in den meisten Fällen überschritten und somit in spürbarem Umfang eine echte Selbstbeteiligung eingefordert wird..$^{48}$ Dieser Mangel wird allerdings durch die Ausnahmeregeln vom Selbstbeteiligungstarif gemildert:

Bei der Aufstellung der Kriterien ist dargelegt worden, daß eine Selbstbeteiligung aufgrund ihres prämienmindernden Effekts dann als erwünscht gelten kann, wenn der Einkommensnutzen infolge der Pflegebedürftigkeit sinkt. Eine Vollabsicherung, die dafür sorgt, daß die Einkommensposition im Schadensfall intakt bleibt, ist unter dieser Voraussetzung nicht optimal. Es wurde dennoch für den Verzicht auf Selbstbeteiligung als Ideallösung plädiert. Dies mit dem Argument, daß ein "komplementäres" Verhältnis zwischen Gesundheitszustand und Einkommen keineswegs generell unterstellt werden

\footnotetext{
${ }^{47}$ siehe Kapitel 5.3

${ }^{48}$ vgl. Ziekenfondsraad (1987), S. 8
} 
kann und der einzelne auch nicht abschätzen kann, wie in dieser Hinsicht sein eigener Pflegefall gelagert sein wird.

Die Regelungen der niederländischen Pflegeversicherung lassen sich nun so interpretieren, daß man ebendie Fälle von der hohen Selbstbeteiligung auszunehmen versucht, in denen ein verringerter Verwendungsnutzen des Einkommens nicht angenommen werden kann. Dies gilt insbesondere für diejenigen Menschen, die bei Eintritt der Hilflosigkeit mit anderen zusammenleben. Ist das Einkommen zuvor gemeinschaftlich verausgabt worden, so wird durch den Weggang eines Beteiligten der Bedarf oftmals nur unwesentlich sinken und gleichzeitig evtl. um "Besuchskosten" und ähnliches erhöht. Per saldo wird das Einkommen, so wird daher offenbar unterstellt, noch im (annähernd) gleichen Umfang benötigt wie zuvor.

Anders im Fall alleinlebender Personen, die nachhaltig pflegebedürftig werden. Hier wird ein Wegfall wesentlicher Verwendungsmöglichkeiten für das Einkommen angenommen, so daß der Einkommensnutzen deutlich zurückgeht. ${ }^{49}$ Eine Selbstbeteiligung ist dann unschädlich. Aber auch hier wird noch differenziert: Bei Aussicht auf Rückkehr aus dem Pflegeheim erhöht sich der von der Selbstbeteiligung unberührte Freibetrag etwa um die Kosten, die aus der Beibehaltung der Privatwohnung erwachsen. ${ }^{50}$

Freilich sind die verwandten Kriterien relativ grob. Es ist sehr wohl vorstellbar, daß je nach Sachlage im Einzelfall auch das Einkommen einer nicht allein lebenden Person mit Eintritt der Pflegebedürftigkeit weitgehend "überflüssig" wird und daß umgekehrt auch mancher alleinstehende Empfänger sein Einkommen trotz Pflegebedürftigkeit noch nutzbringend $\mathrm{zu}$ verwenden weiß..$^{51}$ Als ungefähre Annäherung an den unterschiedlichen Nutzen des Einkommens im Pflegefall kann die differenzierte Gestaltung des Selbstbehalts dennoch angesehen werden.

\footnotetext{
${ }^{49}$ Wie an anderer Stelle erwähnt, wurde in der Debatte um die Erhöhung des Selbstbehalts mit der Vorstellung argumentiert, daß die betroffenen Pflegebedürftigen ihr Einkommen nurmehr zum Aufbau von Vermögen verwenden (könnten), das sie selbst nicht mehr verbrauchen würden.

so vgl. Informatiecentrum Zorg en Welzijn (1992), S. 20

${ }^{51}$ Wie erwähnt ist die massive Ungleichbehandlung anhand des Haushaltsstatus für juristisch unhaltbar erklärt worden.
} 
Im Sinne dieser Argumentation ist es allerdings problematisch, daß das heutige Niveau der Selbstbeteiligung erst als Resultat mehrerer drastischer Erhöhungen im Lauf der Zeit erreicht worden ist. Wer etwa zum Zeitpunkt einer solchen Erhöhung pflegebedürftig wird, kommt selbst nicht mehr in den Genuß der durch sie bewirkten Beitragsermäßigung. Vielmehr werden in solchen Fällen Anspruchsbestandteile gestrichen, für die die Betroffenen bereits gezahlt zu haben glaubten. ${ }^{52}$

Die Selbstbeteiligung im ambulanten Bereich bedarf in ihrer bisherigen Form im hier behandelten Zusammenhang kaum der gesonderten Erwähnung. Wird die häusliche Pflege praktisch kostenfrei gewährt, so bietet im hauswirtschaftlichen Bereich die Einkommensabhängigkeit des maximalen Eigenbeitrags eine Gewähr dafür, daß die Schutzziele im wesentlichen gewahrt bleiben. Mit etwa hfl 10 pro Stunde ist der Selbstbehalt dabei so festgesetzt, daß die Versicherung nach wie vor den größeren Teil der Kosten übernimmt.

Zusammenfassend kann angesichts der aufgeführten Merkmale die von der Selbstbeteiligung ausgehende Zielabweichung der Pflegeversicherung zum heutigen Zeitpunkt als gering angesehen werden. Die zuvor dargestellten Schwierigkeiten bei der tatsächlichen Erlangung dem Grunde nach zugestandener Dienste sind unter dem Aspekt der Forderung nach angemessenem Leistungsumfang das gravierendere Problem.

\subsubsection{Leistungsinhalte und Leistungsform}

Die niederländische Pflegeversicherung gewährt traditionell ${ }^{53}$ ausschließlich Sachleistungen. Bei der Erörterung des Kriterienkatalogs wurde kein generelles Urteil darüber gefällt, ob dieses Konzept einem teilweise oder vollständig auf Geldleistungen orientierten unter Effektivitätsgesichtspunkten vorzuziehen ist. Den Vorzügen des Naturalsystems hinsichtlich des Klientenschutzes steht die im Grundsatz größere Flexibilität von Geldleistungen im Sinne der Autonomie des Empfängers gegenüber. Die Diskussion dieser Vor- und Nachteile kann hier nicht mit empirischen Mitteln vertieft werden, da Erfahrungen mit monetären Transfers in den Niederlanden zu keinem Zeitpunkt gesammelt worden sind.

\footnotetext{
\$2 vgl. Ziekenfondsraad (1987), S. 6

${ }^{53} \mathrm{Zu}$ den Plänen für eine Auflockerung dieses Prinzips siehe Abschnitt 5.5.1.3.
} 
Nur rein spekulativ wäre in diesem Sinn auch die Frage zu beantworten, ob die eben geschilderte Knappheit der verfügbaren Leistungen im Effekt größer oder geringer wäre, wenn sich die Niederlande für Geldtransfers entschieden hätten. Unterstellt werden kann aber immerhin, daß sie unter dieser Voraussetzung eine andere Form angenommen hätte. Als notwendig angesehene Maßnahmen zur Kostendämpfung könnten direkt bei der Bemessung der Geldleistungen ansetzen, wodurch sich die restriktive Kontrolle der Angebotskapazitäten erübrigen würde. Entscheidend für den Erhalt angemessener Leistungen wäre dann vermutlich nicht mehr, wie oben geschildert, die besondere Dringlichkeit des Bedarfs (oder auch der glückliche Zufall), sondern eher die Fähigkeit der Klienten, das Pflegegeld aus eigenen Mitteln aufzustocken. Auch dadurch würde das Schutzziel verletzt.

Konkret geprüft werden kann hier nur, inwieweit das niederländische System die in Kapitel 3 genannten Bedingungen erfüllt, unter denen die Sachform aller Leistungen als zielgerecht und überlegen gelten kann. Diese lassen sich in den Merkmalen Angebotsbreite, Flexibilität, Innovationsfähigkeit und Wettbewerb zusammenfassen:

\section{a) Angebotsbreite}

Seit der Ausdehnung des Leistungsspektrums auf die Bereiche der ambulanten Pflege und der hauswirtschaftlichen Unterstützung deckt die Pflegeversicherung die gesamte Breite des möglichen Bedarfs ab. Insoweit wird der dargelegten Unterschiedlichkeit des von Fall zu Fall Benötigten und Gewollten Rechnung getragen. Hervorzuheben ist innerhalb der abgestuften Leistungen die Möglichkeit der "Tagesbehandlung" in Pflegeheimen, die in den Niederlanden relativ stark ausgebaut ist und eine für Grenzfälle zwischen ambulantem und stationärem Bedarf bedeutsame Option darstellt. Andererseits ergibt sich an dieser Nahtstelle zwischen den Pflegesektoren eine mögliche Lücke aus der erwähnten Beschränkung ambulanter Pflege auf zweieinhalb Stunden pro Tag. Dieses Limit kann im Einzelfall Unterversorgung oder auch das Gegenteil in Form unnötiger Heimeinweisungen bewirken. ${ }^{54}$

\footnotetext{
${ }^{54}$ Im übernächsten Anschnitt ist von ersten Versuchen die Rede, dieses Problem zu lösen.
} 
b) Flexibilität

Auch diese Forderung ist im Sinne der Möglichkeit, bei Zustandsveränderungen zwischen den Stufen des Pflegeangebots in beide Richtungen zu wechseln, grundsätzlich erfüllt. So wird etwa die Indikation zur stationären Unterbringung regelmäßig überprüft, um ggfs. eine adäquatere Lösung zu finden. Daß dies in der Praxis in einigem Umfang zum Erfolg führt, zeigt die Entlassungsbilanz der Heime: Im Jahre 1987 kehrten fast $27 \%$ der Inhaber von Heimplätzen im somatischen Bereich nach Hause zurück; im der Psychogeriatrie waren es allerdings weniger als vier Prozent. ${ }^{55}$ Besonders große Fluktuation herrscht - seiner Brückenfunktion gemäß - im Bereich der Tagesbehandlung: Jeder Platz wird im Laufe eines Jahres von durchschnittlich drei Patienten eingenommen. ${ }^{56}$ Von denjenigen, die eine solche Behandlung verlassen, gehen gut $40 \%$ in die vollstationäre Behandlung über, ein Fünftel kehrt in die häusliche Umgebung zurück. $^{57}$

Ein Hindernis für die Beweglichkeit des Leistungsprozesses entlang des Bedarfs stellen indes die beschriebenen Kapazitätsengpässe dar. Die Angaben dazu brauchen hier nicht wiederholt zu werden.

\section{c) Innovationsfähigkeit und Wettbewerb}

Die Entwicklung zusätzlicher Betreuungsangebote in Reaktion auf veränderte Bedarfslagen ist im niederländischen Pflegewesen zumindest ansatzweise durchaus zu beobachten. Ein Beispiel ist die Einrichtung der Rufbereitschaft durch die Träger der ambulanten Dienste. Sie ist von großer Bedeutung für Personen, die im allgemeinen nicht stark pflegebedürftig sind, deren Zustand aber schwankt bzw. gefährdet erscheint. Ebenso haben sich in letzter Zeit verstärkt verschiedenartige Angebote zur zeitweiligen Aufnahme ins Heim herausgebildet. ${ }^{58}$ Sie sind allerdings nicht überall vorhanden und zeigen somit auch die Grenzen der Innovationsfreude: Die Unterbringung derartiger Pflegeformen im Finanzierungssystem bereitet nach Auskunft der Heimbetreiber mit-

\footnotetext{
55 errechnet aus Angaben bei J.M. Spaan (1989), S. 53 und 14

s6 ebenda, S. 49

${ }^{57}$ N.J.H.W. van Weert (1991), S. 5

${ }^{58}$ siehe G. ten Haaft (1988). Genannt werden dort im einzelnen: die Aufnahme wegen Ferien privater Betreuer/innen, die regelmäßige Wochenend- bzw. Werktagsaufnahme, die Intervall-Aufnahme, die Krisenaufnahme und die Nachtaufnahme.
} 
unter Schwierigkeiten oder findet im Rahmen sehr zurückhaltend gewährter Sondermittel statt. ${ }^{59}$

Besondere Beachtung verdient schließlich der Bereich der "ergänzenden Hauspflege", durch die die oben erwähnte Lücke zwischen der maximal zugestandenen ambulanten Pflege und der (teil-)stationären Unterbringung geschlossen werden soll. Hier wird zunehmend die Möglichkeit eingeräumt, weitere Pflegeeinheiten in Anspruch zu nehmen, an deren Bereitstellung sich neben den üblichen ambulanten Trägern auch die Heime und sonstige Kliniken sowie private Anbieter beteiligen. ${ }^{60}$ Den mit der Ausführung des AWBZ betrauten Versicherungen wird aus Beitragsmitteln ein spezieller Etat für die Organisation solcher Modelle zur Verfügung gestellt. Die Reichweite der neuen Pflegeform ist nach den letzten mir zugänglichen Daten noch gering, aber wachsend: Die Anzahl der betreuten Patienten lag 1987 bei 900, im Jahr darauf bei 3000 Personen. Ein deutlicher Schwerpunkt liegt dabei bislang auf Patienten im Endstadium, denen so die Möglichkeit gegeben werden soll, zu Hause zu sterben. ${ }^{61}$

Der letzte Bereich ist auch deswegen bemerkenswert, weil dort anders als in den herkömmlichen Pflegezweigen eine Vielfalt von Anbietern tätig ist. Die Auswirkungen dieser Tatsache dürften im Sinne von erweiterten direkten Wahlmöglichkeiten der Klienten allerdings schon deswegen gering sein, weil es sich typischerweise um Schwerstkranke handelt. Eher bieten sich hier für die Versicherungsorgane Möglichkeiten, leistungsfähige und kostengünstige Anbieter auszuwählen.

Außerhalb der "ergänzenden Hauspflege" fällt ein Wettbewerb zwischen Leistungserbringern als mögliches Element einer Sicherstellung differenzierter Möglichkeiten zur Deckung unterschiedlichen Pflegebedarfs im ambulanten Sektor weitgehend aus, da die Erstellung von Versicherungsleistungen hier in den Händen einer Organisation liegt. Bei der Wahl eines Pflegeheims können individuelle Wünsche geltend gemacht werden; sofern aber die Nachfrager mit der beschriebenen Knappheit an Plätzen konfrontiert sind, ist das Wahlrecht faktisch entwertet. Ein Wechsel des Pflegeheims ist

\footnotetext{
${ }^{59}$ siehe ebenda, S. 758f.

${ }^{60}$ vgl. Tweede Kamer (1992), S. $153 \mathrm{f}$.

${ }^{61}$ vgl. Nationale Kruisvereniging (1989), S. 35
} 
ebenfalls grundsätzlich möglich, kommt aber eher selten vor: Bei einer Gesamtkapazität von rd. 50.000 Betten werden jährlich rd. 1.000 solcher Umzüge gezählt. ${ }^{62}$

Gerade für den Bereich der häuslichen Pflege, in dem die subjektiven und sachlichen Gestaltungsmöglichkeiten als relativ ausgeprägt unterstellt werden können, ist in den Niederlanden gelegentlich der Übergang auf ein System von Geldleistungen gefordert worden, um dem "Erhalt von Selbständigkeit und dem Selbstbestimmungrecht des Klienten" besser Rechnung tragen zu können. ${ }^{63}$ Vielversprechend ist ein solcher Gedanke auch angesichts der Tatsache, daß freie Anbieter bereits heute neben der offiziellen Organisation existieren, obwohl ihre Dienste im allgemeinen privat finanziert werden müssen. Offenbar fungieren sie nicht nur als Lückenbüßer zur Ergänzung des knappen Umfangs der Versicherungsleistungen, sondern werden mitunter auch aufgrund ihrer spezifischen Dienstleistungen der "Normalpflege" vorgezogen. ${ }^{64} \mathrm{Nach}$ Auskunft des Ziekenfondsraad vom März 1994 wird mit dem bei GOUDRIAAN/WENNINK diskutierten Budget-Verfahren, also dem Ersatz der Sachleistungen durch eine pauschale Geldleistung in entsprechender Höhe, inzwischen auf freiwilliger Basis experimentiert.

Zusammenfassend läßt sich sagen, daß sich das Leistungswesen der niederländischen Pflegeversicherung aufgrund der Breite und zunehmenden Differenziertheit des Leistungsspektrums insgesamt als funktionstüchtig und reaktionsfähig gezeigt hat. Es erscheint zweifelhaft, ob der auf diese Weise vollzogene Aufbau einer flächendeckenden und recht vielfältigen pflegerischen Infrastruktur auch mittels Geldleistungen gelungen wäre, von denen ja angenommen werden muß, daß ein Teil in zweckfremde Verwendungen abfließt.

Gleichwohl zeigen sich auf dem heute erreichten Stand auch die Grenzen der Flexibilität eines derartigen Systems. Probleme liegen nicht nur in der für Sachleistungen typischen Standardisierung von Angeboten, sondern auch in der administrierten Leistungsknappheit, die prinzipiell vorhandene Entscheidungsfreiräume beschneidet und sich daher auch auf der hier behandelten Zielebene schädlich auswirkt. Eine Lockerung der strikten

\footnotetext{
${ }^{62}$ J.M. Spaan (1989), S. $52 \mathrm{f}$

${ }^{63}$ F.G.W. Goudriaan/J. Wennink (1992), S. 4

${ }^{64}$ siehe näheres bei W. Reijmerink (1990), insbes. S. 80. Einer dort zitierten Untersuchung zufolge entscheiden sich immerhin $18 \%$ der Hilfsbedürftigen für eine "kommerzielle" Lösung anstelle der regulären Leistungen.
} 
Orientierung auf Sachleistungen birgt daher je nach Ausgestaltung zumindest in Teilbereichen Chancen auf eine Verbesserung der Effektivität.

Wesentlich für die Beurteilung des Sachleistungswesens ist auch die damit erreichte Qualität der Pflegedienste, die nunmehr zur Sprache kommt.

\subsubsection{Leistungsqualität}

a) stationärer Sektor

Als wesentlichster und am ehesten operationalisierbarer Indikator für die Pflegequalität ist bei der Aufstellung von Zielkriterien die Personalausstattung der Einrichtungen benannt worden. Hierüber liegen in den Niederlanden recht präzise, differenzierte und jährlich aktualisierte Statistiken vor. Aus ihnen geht hervor, daß im Jahr 1990 für jeweils 100 belegte Betten in Pflegeheimen Personal im Umfang von rd. 117 Vollzeitkräften zur Verfügung stand ${ }^{65}$; der Wert für 1989 betrug 116,6 und wird in Tabelle 6.1 nach Heimtypen und Art der Arbeitskräfte aufgeschlüsselt.

Die dort ausgewiesene Besetzung erscheint recht großzügig, auch wenn sich die Arbeitszeit der im Pflegeheim Tätigen natürlich so auf den pausenlosen Dauerbetrieb verteilt, daß zu jedem Zeitpunkt nur ein geringer Teil der Gesamtbelegschaft anwesend ist. Um den ersten Eindruck zu prüfen, seien zum Vergleich in der Bundesrepublik Deutschland gültige Personalschlüssel herangezogen.

Dabei ist allerdings vorauszuschicken, daß entsprechende Werte in der Bundesrepublik nicht zentral und regelmäßig erhoben werden und in Teilen auf Schätzungen basieren.

Ein neuerer Versuch in dieser Richtung ist von einer Arbeitsgruppe der Bundesarbeitsgemeinschaft der höheren Kommunalverbände unternommen worden und bezieht sich auf das Jahr 1990. ${ }^{66}$ Auf Basis von Angaben aus sieben westlichen Bundesländern wird dort eine Besetzung von 58,9 Vollzeitkräften pro 100 Betten im Pflegeheim errech-

\footnotetext{
${ }^{65}$ Ermittelt als gewogenes Mittel aus den Werten pro Kategorie in NZI (1990), S. 65ff. Teilzeitkräfte sind umgerechnet.
}

${ }^{66}$ W. Jopen (1990) 
net. ${ }^{67}$ Dies ist ziemlich genau die Hälfte des niederländischen Niveaus. Kleinere Vergleichbarkeitsmängel, die hier nicht behoben werden können ${ }^{68}$, dürften die enorme Diskrepanz allenfalls unwesentlich überzeichnen.

\section{Tabelle 6.1}

\section{Personal in Pflegeheimen pro 100 belegte Betten 1989}

\begin{tabular}{||l|c|c|c||}
\cline { 2 - 4 } \multicolumn{1}{l|}{} & Somatik & $\begin{array}{c}\text { Psychogeria- } \\
\text { trie }\end{array}$ & Kombinierte \\
\hline $\begin{array}{l}\text { Verwaltung/Haus- } \\
\text { wirtschaft }\end{array}$ & 28,2 & 24,6 & 27,5 \\
\hline $\begin{array}{l}\text { Pflegepersonal im } \\
\text { engen Sinn }\end{array}$ & 71,9 & 72,1 & 74,8 \\
\hline $\begin{array}{l}\text { Medizinisches und } \\
\text { paramedizinisches } \\
\text { Hilfspersonal }\end{array}$ & 9,1 & 8,1 & 9,0 \\
\hline $\begin{array}{l}\text { Medizinischer und } \\
\text { sozialwiss. Stab }\end{array}$ & 1,2 & 1,4 & 1,4 \\
\hline $\begin{array}{l}\text { Sonstige inkl. Prakti- } \\
\text { kanten }\end{array}$ & 5,9 & 5,9 & 6,0 \\
\hline S u m m e & 116,4 & 112,0 & 118,7 \\
\hline
\end{tabular}

Quelle: $\quad$ NZI (1991) Teil 1-3, jeweils S. 12

${ }^{67}$ ebenda, S. 355

68 So werden die Bewohner von deutschen Pflegeheimen vielfach von außerhalb (nämlich durch Hausärzte) medizinisch betreut, während in niederländischen Heimen regelmäßig auch Ärzte tätig sind. Da die betroffenen Personalgruppen in der dritten und vierten Zeile der Tabelle aber so abgegrenzt sind, daß sie auch Funktionen enthalten, die in deutschen Heimen ebenso vorkommen, wurde davon abgesehen, sie herauszurechnen. 
Wird die Betrachtung auf das direkt bewohnerbezogene Pflege- und Funktionspersonal konzentriert, fällt der Unterschied zwischen beiden Ländern noch etwas deutlicher aus. Hier wird für die Bundesrepublik ein Wert von 38,7 Kräften auf 100 Betten genannt. ${ }^{69}$ Das übrige Personal fällt in der deutschen Einteilung unter die Rubriken "Wirtschaftspersonal" sowie "Leitung und Verwaltung". Um einen Vergleichswert für die Niederlande zu erhalten, werden von den Werten in Tabelle 6.1 die erste und - sicherheitshalber auch die unklare - letzte Zeile abgezogen. Die verbleibende Pflegepersonalquote von 83,3 übersteigt die deutsche um $115 \%$.

Zunächst wirft die Größe des Unterschieds die Frage auf, ob sich dafür auf seiten der Bewohnerschaft von Pflegeheimen Erklärungen finden lassen. Ihr ist das Kuratorium Deutsche Altershilfe 1980 anläßlich einer Studienreise in die Niederlande nachgegangen, bei der - auf beiderseits niedrigerem Niveau - noch größere Unterschiede in der Personalausstattung pro Patient festgestellt worden waren. ${ }^{70}$ Es wurde geprüft, ob hinsichtlich der Altersstruktur oder der mittleren Fallschwere ebenfalls Diskrepanzen vorlagen, die einen unterschiedlichen Pflegebedarf hätten begründen können. Der Befund war in beiden Punkten negativ: Bei identischem Altersprofil ${ }^{11}$ waren die Beeinträchtigungen bei den niederländischen Heimbewohnern eher noch etwas geringer als in Westdeutschland. ${ }^{72}$

Jedenfalls hinsichtlich des Altersaufbaus gilt diese Feststellung auch heute noch. Die im Laufe der 80er Jahre eingetretene deutliche Verschiebung zugunsten Hochbetagter scheint in beiden Ländern parallel verlaufen zu sein. Wird das Durchschnittsalter von Pflegeheimbewohnern für 1990 in Deutschland (West) mit 80 Jahren angegeben $^{73}$, so beträgt es in den Niederlanden rd. 80,5 Jahre. ${ }^{74}$ Der Anteil der mindestens 80jährigen liegt in Deutschland mit 63\% sogar etwas höher als in den Niederlanden

\footnotetext{
${ }^{69}$ Ebenda. Siehe für aktuelle Daten auch J. Allemeyer (1994), S. 496f. Bei den dort aufgeführten Stellenschlüsseln wird die Schwelle von 50 Pflegekräften auf 100 Plätze in keinem Bundesland für keine Pflegestufe überschritten.

${ }^{70}$ siehe M. v.Rötel et al. (1980), S. 12f.

${ }^{71}$ Verglichen wurden die niederländischen Werte mangels bundesweiter Erhebungen mit denen des Bundeslandes Baden-Württemberg (siehe ebd., S. 5).

72 siehe ebenda, S. $6 \mathrm{f}$.

${ }^{73}$ W. Krug/G. Reh (1992), S. 27

${ }^{74}$ Errechnet aus Angaben bei SIG (1992), S. 65 und S. 80
} 
$(61,3 \%) .{ }^{75}$ Von daher gibt es keinen Anhaltspunkt dafür, daß deutsche Heimbewohner deutlich weniger hilfebedürftig sein sollten als niederländische. Für einen direkten Vergleich des Zustandsprofils fehlt die Datengrundlage.

Liegt der Grund für die divergierenden Personalschlüssel also nicht in den objektiven Anforderungen der jeweiligen Patienten, kommt außer echten Qualitätsunterschieden allenfalls noch die These in Betracht, daß im niederländischen Pflegesektor weitaus unproduktiver gearbeitet werde. Da aber - wie bereits ausgeführt - ein technischer Produktivitätsbegriff in der Pflege nicht anwendbar ist ${ }^{76}$ und keine größeren Rationalisierungspotentiale bestehen, müßte dies letztlich bedeuten, daß das niederländische Heimpersonal in großem Maßstab unterbeschäftigt ist. Im oben genannten Umfang ist das nicht plausibel. Zudem erscheint eine solche Vermutung kaum vereinbar damit, daß die niederländische Regierung dem Pflegepersonal eine hohe Arbeitsbelastung attestiert, die durch Neueinstellungen gemildert werden soll. ${ }^{77}$

Es kann mithin angenommen werden, daß im Vergleich zur Bundesrepublik in den Niederlanden generell weitaus sorgfältiger und umfassender gepflegt wird. ${ }^{78}$ Es sei nochmals betont, daß diese Feststellung nicht mit einer direkten inhaltlichen Qualitätsmessung verwechselt werden darf. ${ }^{79}$ Eine solche kann hier nicht vorgenommen werden. Aufführen lassen sich aber einige ausgewählte Indizien dafür, daß die relativ gute Personalausstattung mit Erfolgen im Bereich des "Output" korrespondiert. Beweiskräftig sind diese Anzeichen allerdings nicht:

\footnotetext{
${ }^{75}$ In denen 70er Jahren gehörten zu dieser Altersgruppe in beiden Ländern erst rd. $45 \%$ (siehe M. v.Rötel et al. (1980), S. 5).

${ }^{76}$ Zur Erläuterung: Es ist nicht sinnvoll, etwa den Zeitbedarf für bestimmte pflegerische Verrichtungen zu minimieren. Die Tatsache, daß sie behutsam und in Ruhe vorgenommen werden können, ist vielmehr selbst ein qualitatives Leistungsmerkmal. Ein Waschvorgang unter Zeitdruck ist selbst bei gleichem physischem Resultat als "Produkt" nicht identisch mit einem geruhsam vollzogenen.

$n$ siehe Tweede Kamer (1992), S. 127

${ }^{78}$ Ergänzend könnte hier auf das Qualifikationsniveau der Pflegekräfte eingegangen werden. Wegen der unübersichtlichen Datenlage und fehlenden Vergleichsmöglichkeiten wird darauf verzichtet. Nach überschlägigen Berechnungen handelt es sich bei den Pflegekräften in den Niederlanden zu über $80 \%$ um Personen mit einschlägigem Ausbildungsabschluß (siehe NZI (1991), Bände 1-3, insbes. jeweils Tab. 21).

${ }^{79}$ Für Ansätze dazu siehe etwa S. Görres (1993), S. 12, der betont, daß die Erfüllung personeller und sonstiger Ressourcenstandards "eine notwendige aber keine hinreichende Bedingung für eine qualitativ angemessene Versorgung" darstellt.
} 
1) Die erwähnte Studie des Kuratoriums Deutsche Altershilfe weist für die Mitte der 70er Jahre einen auffälligen Unterschied zwischen den Niederlanden und Deutschland beim Anteil der bettlägerigen Patienten aus. Konnte er in den Niederlanden zwischen 1964 und 1976 von 16 auf $5 \%$ gesenkt werden, wird er für die Bundesrepublik mit $28 \%$ angegeben. Dies wird als bemerkenswert vor allem deswegen eingestuft, da sich die Störungs- bzw. Fähigkeitsprofile der Heimbewohner ansonsten nur unwesentlich zwischen beiden Ländern unterscheiden. "Wahrscheinlich ist Bettlägerigkeit weniger ein objektives Merkmal der Pflegebedürftigkeit als vielmehr ein Indikator für (...) Pflegeverhalten". ${ }^{80}$

2) Bereits genannt wurden die auffallend hohen "Rückkehrquoten" insbesondere im somatischen Bereich niederländischer Pflegeheime. $\mathrm{Zu}$ würdigen sind sie auf dem Hintergrund der Tatsache, daß wegen der Knappheit an Plätzen und dem Verfahren ihrer Vergabe die durchschnittliche Fallschwere der Aufgenommenen im Lauf der Zeit deutlich zugenommen hat. ${ }^{81}$ Vergleichswerte für Deutschland liegen mir nicht vor; nach allgemeinem Eindruck gelten Pflegeheime hierzulande als typische "Endstation".

3) Hingewiesen sei zuletzt auf eine demographische Auffälligkeit, die mit einer überlegenen Pflegekultur in den Niederlanden zusammenhängen könnte. Die Restlebenserwartung der 80jährigen lag dort nach OECD-Angaben Ende der 80er Jahre um über 10 Prozent höher als in der alten Bundesrepublik. Die Niederlande rangieren damit an siebter Stelle im Vergleich der 23 OECD-Staaten, die Bundesrepublik an fünftletzter. ${ }^{82}$ Bemerkenswert ist nun, daß sich diese Diskrepanz erst nach 1970 bei genau gleicher Ausgangsbasis in beiden Ländern eingestellt hat. ${ }^{83}$ Die Gesamtlebenserwartung ist dagegen im gleichen Zeitraum in Deutschland wesentlich schneller angestiegen als in den Niederlanden, so daß das Phänomen einer spezifischen Erklärung in bezug auf die alten Menschen bedarf. Daß unterschiedliche Pflegeangebote und qualitäten seit Einführung der Pflegeversicherung in den Niederlanden dabei eine Rolle gespielt haben, ist angesichts des hohen Anteils von Pflegebedürftigen unter den

\footnotetext{
${ }^{80}$ M. v.Rötel et al. (1980), S. 6

${ }^{81}$ siehe NZI (1989), S. 10

${ }^{82}$ H. Oxley (1992), S. 66

${ }^{83}$ Die durchschnittlichen jährlichen Wachstumsraten finden sich ebenda, S. 72; auf das Niveau von 1970 wurde mit ihrer Hilfe zurückgerechnet.
} 
Hochbetagten immerhin eine naheliegende Vermutung. Um einen solchen Zusammenhang zu erhärten, bedürfte es freilich einer gesonderten Untersuchung.

Spricht also die Personalausstattung recht deutlich für ein hohes Qualitätsniveau der Pflegeheime, so erscheint die räumliche Situation - jedenfalls nach dem Stand von 1987 - weniger zufriedenstellend: Zwar hat über die Hälfte der Zimmer nicht mehr als zwei Betten, mehr als die Hälfte der Patienten aber lebt in Räumen mit vier und mehr Betten und immerhin 18\% der Plätze entfallen auf Sechsbettzimmer. ${ }^{84}$

b) ambulanter Sektor

Auf diesen Bereich kann mangels (Vergleichs-)Maßstäben nur beschränkt eingegangen werden. Wie bereits dargestellt ${ }^{85}$ steht er in letzter Zeit im Zeichen einer zunehmenden Spannung zwischen deutlich steigender Nachfrage und knapp gehaltenen Mitteln. Das gilt sowohl für die häusliche Pflege als auch für die hauswirtschaftlichen Hilfen. Es ist anzunehmen, daß dieser Zustand neben der Zugänglichkeit auch die Qualität der Dienste beeinträchtigt.

So berichtet der Dachverband der Anbieter zum Beispiel von einem Rückgang der präventiven Aktivitäten und von Frustration unter den Mitarbeiterinnen angesichts steigender Belastung mit Intensivpflege bei gleichzeitig verschlechterter relativer Einkommensposition. ${ }^{86}$ Die Rekrutierung neuen Personals werde dadurch erschwert. ${ }^{87}$ Gleichzeitig versuchen die Anbieter und ihre Organisationen, ihrer wachsenden Bedeutung bei der Dämpfung des Bedarfs an stationärer Pflege durch Erhöhung der pflegerischen und administrativen Flexibilität gerecht $\mathrm{zu}$ werden. ${ }^{88}$

Es gibt also Anzeichen dafür, daß der Versorgungsstandard im ambulanten Sektor von Verfallserscheinungen erfaßt oder bedroht ist. Ein Vergleich mit Leistungniveaus außerhalb der Niederlande, wie er für die stationäre Pflege im vorigen Abschnitt angestellt wurde, muß hier aufgrund fehlender Daten und Maßstäbe unterbleiben.

\footnotetext{
${ }^{84}$ J.M. Spaan (1989), S. 44

${ }^{85}$ siehe Kapitel 6.1.3.(b)

${ }^{86}$ vgl. Nationale Kruisvereniging (1989), S. 13 bzw. S. 36

${ }^{87}$ LVT (1993), S. 30

${ }^{88}$ siehe ebenda
} 


\subsection{Pflege als Grundbedürfnis - zur Bewertung anhand des modifizierten Zielkatalogs}

Im Anschluß an die Anwendung der einzelnen Zielkomponenten bleibt nun noch zu prüfen, inwieweit die niederländische Pflegeversicherung dem modifizierten Zielkatalog genügt, wie er sich aus dem Postulat eines für alle gleichen Zugangs zu benötigten Pflegeleistungen ergibt. Es ist darauf hingewiesen worden, daß dieser Aspekt bei der Einrichtung der Versicherung eine Rolle gespielt zu haben scheint. Auch in der Diskussion um die Umgestaltung der Krankenversicherung hat sich gezeigt, daß ein solcher Gleichheitsanspruch zumindest als Prinzip in den Niederlanden weitgehend konsensfähig ist.

Bei der Pflegeversicherung ist zumindest in Hinblick auf die stationäre Versorgung weitestgehend sichergestellt, daß die wirtschaftliche Situation des Einzelnen ohne Einfluß auf die Verläßlichkeit und den qualitativen Gehalt der Bedarfsdeckung bleibt. Ein privater Zukauf von Sonderleistungen durch Heimbewohner ist nicht möglich und insofern auch nicht erforderlich, als der Pflegestandard der Heime generell hoch ist und die Kostenerstattung durch die Versicherung sich jeweils nach dem gesamten Pflegepreis richtet. Die Bemessung des Selbstbehalts nach dem Einkommen verhindert systematisch, daß Ärmere aus Kostengründen unter sonst gleichen Umständen eher als Reiche darauf verzichten, Pflegebedarf geltend zu machen.

Abstriche vom egalitären Bild der pflegerischen Versorgung sind indes dann zu machen, wenn die Kapazitätslücken in Betracht gezogen werden. Diese sind offenbar (bislang) nicht so groß, als daß sich ein "zweiter Markt" für frei finanzierte Heimpflege herausgebildet hätte. Sehr wohl aber macht es für Bedürftige, die in häuslicher Umgebung auf die Aufnahme in ein Pflegeheim warten, einen Unterschied, ob sie finanziell in der Lage sind, ambulante Leistungen bei privaten Anbietern zur Ergänzung der seitens der Versicherung gewährten Dienste zu erwerben. Es sei daran erinnert, daß die ambulante Pflege (ohne die hauswirtschaftliche Komponente) als Versicherungsleistung im Regelfall auf maximal drei Besuche und zweieinhalb Stunden pro Tag begrenzt ist und je nach Auslastung der Dienste im Einzelfall faktisch noch strenger limitiert sein kann. Die inzwischen für bestimmte Fälle mögliche Aufhebung der Leistungsgrenze unter dem Titel der "intensiven Hauspflege" findet bisher nur in einem engen budgetären Gesamtrahmen statt. Von der weiteren Entwicklung in diesem Punkt und von der künftigen Kapazitätspolitik hängt es ab, in welchem Ausmaß sich entgegen 
dem noch immer gültigen Anspruch einer bedarfsgerechten Pflege für alle eine Versorgungsstruktur herausbilden wird, die nach Einkommensklassen gestaffelt ist.

\subsection{Zur Bewertung unter Effizienzgesichtspunkten}

Unter dieser Rubrik sind in Kap. 4 die Kriterien der Verläßlichkeit, der Finanzierungsäquivalenz und des moral hazard aufgestellt und erörtert worden. In dieser Reihenfolge werden sie nunmehr auf die niederländische Pflegeversicherung angewandt.

\subsubsection{Verläßlichkeit}

Unter diesem Punkt sind vier Formen von Leistungseinschränkungen unterschieden worden, die den ursprünglich in Aussicht gestellten Versicherungsschutz schmälern können:

- der erschwerte Leistungszugang,

- die verstärkte Selbstbeteiligung,

- die verminderte Leistungsqualität und

- das verkleinerte Leistungspektrum.

Aus der bisherigen Darstellung geht hervor, daß insbesondere die beiden ersten Varianten in der Geschichte der niederländischen Pflegeversicherung eine Rolle gespielt haben. Zunächst seien kurz die beiden übrigen abgehandelt.

Das Qualitätsniveau - indirekt gemessen am Personalbestand - liegt seit Beginn der 70er Jahre in etwa auf dem soeben dargestellten hohen Niveau. Zwischen 1972 und 1989 ist ein sehr langsamer Anstieg in der Zahl der Arbeitskräfte pro Pflegeplatz um insgesamt knapp $10 \%$ zu verzeichnen gewesen. ${ }^{89}$ Da die durchschnittliche Fallschwere in diesem Zeitraum spürbar zugenommen hat, läßt sich dies eventuell als schleichender Rückgang des Versorgungsniveaus interpretieren. Eine solche These ist aber entschieden zu schwach, um eine echte qualitative Minderung des Versicherungsschutzes zu begründen. Von Klagen über Qualitätsmängel im ambulanten Bereich war im vorigen Abschnitt bereits die Rede. Relativiert werden sie im hier angesprochenen Zusammenhang aber dadurch, daß dieser Sektor erst nachträglich und schrittweise in das Versicherungssystem integriert worden ist, so daß ein Vertrauensschutz in Hinblick

${ }^{89}$ W. Groenen/R. van der Wouden (1991), Tab. 6 
auf die Leistungszusage hier bislang weniger schwer wiegt als im stationären Bereich, für den bereits seit Jahrzehnten Beiträge entrichtet werden.

Damit ist bereits die Frage des Leistungsspektrums berührt, das im bisherigen Verlauf der Versicherungsgeschichte deutlich ausgebaut und in keinem Punkt beschnitten worden ist. Im Jahre 1993 war von der Idee zu hören, den erst vor wenigen Jahren eingeführten Anspruch auf Hilfe im Haushalt wieder aus dem Leistungskatalog zu streichen..$^{90}$ Dieses Vorhaben des größeren Partners der damaligen niederländischen Regierungskoalition, dessen Durchsetzung auch in Hinblick auf die erwünschte Entlastung der Heimpflege sehr prekär gewesen wäre, ist aber offenbar einstweilen vom Tisch.

Form und Ausmaß der faktischen Zugangsbeschränkungen sind oben ausführlich zur Sprache gekommen. Hier lassen sich die wohl bedeutendsten Verläßlichkeitsmängel der Pflegeversicherung ausmachen, auch wenn zumindest im stationären Sektor neuerdings gegengesteuert wird. Die Bereitschaft, den infolge der demographischen Entwicklung auftretenden Engpässen durch entsprechende Kapazitätsausweitung zu begegnen, war zumindest in den 80er Jahren nur eingeschränkt erkennbar. Dies gilt offenbar bis heute für den ambulanten Sektor, der grundsätzlich in der Lage wäre, die Konsequenzen einer Knappheit an Heimplätzen vergleichsweise kostengünstig abzufedern. Gerade unter bislang noch nicht akut von Pflegebedürftigkeit Bedrohten besteht also Grund zu einer gewissen Unsicherheit, ob sie im Bedarfsfall auch wirklich und rechtzeitig mit angemessener Versorgung rechnen können.

Differenziert zu werten sind schließlich die genannten Entwicklungen bei der Selbstbeteiligung. Einerseits stellt deren kräftige Ausweitung fraglos eine Einschränkung der Schutzwirkung dar. Andererseits ist sie im Gesetz über die Pflegeversicherung im Grundsatz von vornherein angelegt gewesen. Es läßt sich deshalb die Auffassung vertreten, daß ein umfassender Einkommensschutz nicht als Bestandteil der Leistungszusage anzusehen sei und daher auch nicht unter das Verläßlichkeitsgebot falle. ${ }^{91}$ Im übrigen ist $\mathrm{zu}$ betonen, daß die beiden anderen wesentlichen finanziellen Schutzkomponenten bislang intakt geblieben sind: Einen Schutz vor pflegebedingter

\footnotetext{
${ }^{90}$ siehe S. Baart (1993)

${ }^{91}$ Dies ist freilich eine etwas sophistische Argumentation.
} 
Verarmung gewährleistet die Einkommensabhängigkeit des Eigenbeitrags ebenso wie die Wahrung des Vermögens.

In Zusammenhang mit dem letzteren Punkt läßt sich die "prekäre Überlegenheit" einer Sozialversicherung gegenüber anders finanzierten Systemen unter dem Aspekt der Zuverlässigkeit exemplarisch zeigen. Die im Kriterienkapitel dazu vertretene Auffassung besagt, daß Versicherungsleistungen einerseits erkennbar und regelmäßig sicherer seien als andere, etwa steuerfinanzierte, daß diese Sicherheit andererseits keineswegs absolut ist:

Der für die Sozialversicherung zuständige Staatssekretär hat seit Beginn der 90er Jahre mehrere Anläufe unternommen, für die Selbstbeteiligung an Pflegeleistungen einen Zugriff auch auf das Vermögen der Empfänger zu ermöglichen. ${ }^{92}$ Er hat dabei nicht allein auf fiskalische Zwänge verwiesen, sondern die Notwendigkeit einer Gleichbehandlung von Pflegeheimbewohnern und denjenigen betont, die in - nicht auf Pflege ausgerichteten - Altenheimen leben. Diese nämlich erhalten einen (steuerfinanzierten) staatlichen Kostenzuschuß erst dann, wenn ein eventuelles Vermögen aufgezehrt ist.

Die Regierung ist mit dieser Argumentation nicht durchgedrungen. Die Gegner der Maßnahme haben dabei ausdrücklich auf den Versicherungsgedanken bezug genommen, der eine so einschneidende Veränderung der Leistungsbedingungen verbiete. ${ }^{93}$ Obwohl sich die Niederlande im Jahre 1993 in einer Rezessionsphase befanden, die eine Welle von Sparmaßnahmen im Sozialbereich ausgelöst oder begünstigt hat, ist der Vorstoß zur Selbstbeteiligung schließlich zurückgezogen worden..$^{94} \mathrm{Ob}$ die in diesem Punkt zutage getretene Resistenz der Versicherung auch in Zukunft Bestand haben wird, bleibt abzuwarten.

Von Bedeutung für die Verläßlichkeit der Schutzzusage ist schließlich auch die Akzeptanz der Pflegeversicherung. Unter Hinweis auf die externen Effekte zugunsten jüngerer Generationen war hierzu die These aufgestellt worden, daß die Abschaffung oder fortschreitende Aushöhlung einer einmal eingerichteten Pflegeversicherung kaum befürchtet werden müsse. In einer neueren Meinungsumfrage des "Social and Cultural

\footnotetext{
${ }^{2}$ siehe de Volkskrant vom 17. Juli 1992: Ook thuis-verpleegde moet bermogen gaan aanspreken

${ }^{93}$ So argumentiert etwa der Ziekenfondsraad (1989), S. 8

${ }^{94}$ Telefonische Auskunft des Ziekenfondsraad Ende September 1993. Siehe auch J. Bruinsma (1993).
} 
Planning Office" findet sich dies eindrucksvoll bestätigt: Über 70\% der Teilnehmer sehen die Altenpflege als eine überwiegend staatlich/kollektiv zu lösende Aufgabe an. ${ }^{95}$ Zwischen den Altersgruppen streut das Ergebnis ebensowenig wie in bezug auf die meisten sonstigen Merkmale. Selbst eine Gruppe von Befragten, die durch ihren Wunsch nach einem insgesamt geringeren Umfang öffentlicher Leistungen gekennzeichnet ist, zeigt in der Pflegefrage keine nennenswerte Abweichung vom Durchschnitt.

\subsubsection{Finanzierungsäquivalenz}

Den zur Beitragsgestaltung aufgestellten Forderungen gemäß wird in den Niederlanden nach dem AWBZ, das den Pflegesektor einschließt, ein vom Einkommen abhängiger Beitrag erhoben. ${ }^{96}$ Der Tarif ist proportional und kennt nach der Reform von 1990 neben der oberen auch eine untere Beitragsbemessungsgrenze, die dem allgemeinen einkommensteuerlichen Freibetrag entspricht. Von daher und aufgrund der Einbeziehung aller Einkommensempfänger und aller Einkommensarten entspricht das System von seiner Grundstruktur her dem als pareto-optimal dargestellten Muster zunächst genauer als etwa die deutsche Sozialversicherung.

Was die Proportionalität betrifft, so ist gezeigt worden, daß sie bei schematisch vereinfachter Betrachtung exakt die richtige Lösung darstellt, während bei Berücksichtigung der Mehrstufigkeit des Pflegerisikos eventuell andere Tarifformen vorzuziehen wären, deren Effekt eine relative Entlastung höherer Einkommen sein müßte. Dieses Resultat beruht kurz gesagt darauf, daß die Wahrscheinlichkeit, als stationärer Pflegefall die Versicherungshöchstleistung in Anspruch nehmen zu müssen, naturgemäß geringer ist als die, überhaupt Leistungen zu empfangen.

$\mathrm{Zu}$ beachten ist allerdings die in der Modellanalyse nicht vorgesehene variable Selbstbeteiligung. Wenn sie greift, was in den Niederlanden wie gesagt nur bei Pflegebedürftigen ohne Partner der Fall ist, ergibt sich ein andersartiger Verlauf des Versicherungseffekts in Abhängigkeit von der Einkommensposition im Alter. Abbildung

\footnotetext{
${ }^{95}$ P. Dekker/P. Ester (1993), S. 110. Für die alternativ zur Auswahl gestellten informellen und kommerziellen Lösungswege votierten 19 bzw. $10 \%$.

${ }^{9}$ Die kürzlich hinzugekommene kleine einkommensunabhängige Beitragskomponente, die bei der Darstellung der niederländischen Gesundheitsreform in Kapitel 5.5.1 erwähnt worden ist, bleibt hier außer Betracht, zumal sie - wie gesagt - demnächst wieder entfallen soll.
} 
6.1 zeigt dies skizzenhaft: Sowohl für den Fall ohne Selbstbeteiligung, als auch für die niederländische Lösung ist dort erkennbar, welcher Vorteil sich aus der Versicherung gegenüber der Referenzlage ohne Versicherung, aber mit Mindestsicherung ergibt.

\section{Abbildung 6.1}

Versicherungseffekt mit variabler Selbstbeteiligung bei einheitlichem Pflegesatz P

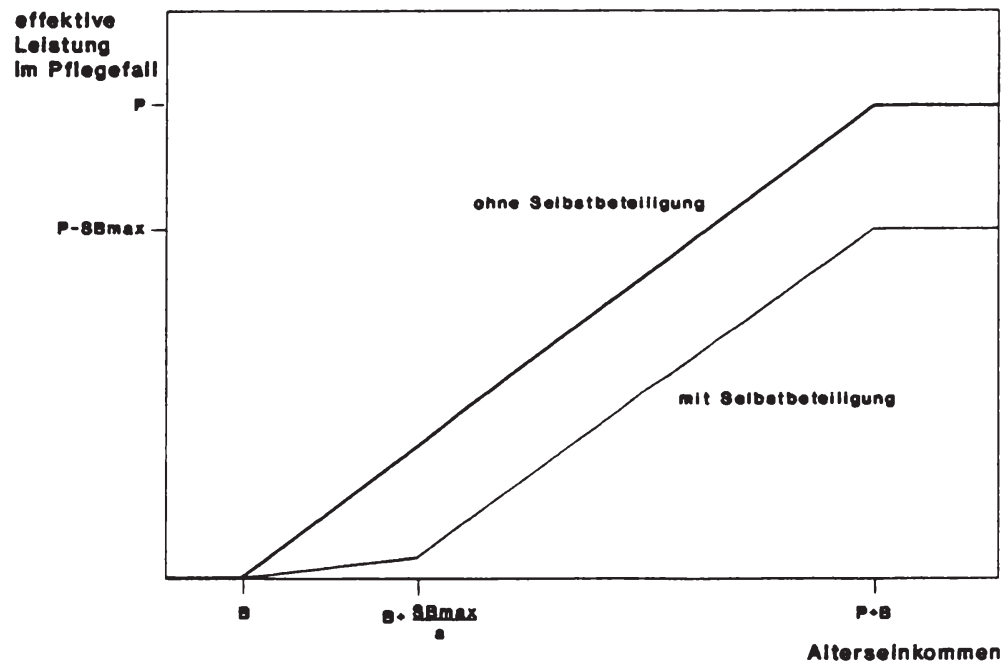

eigene Darstellung

Schon für den dort unterstellten einfacheren Fall eines einheitlichen Pflegekostensatzes zerfällt die Zone, in der dieser Vorteil zunimmt, nun in zwei Bereiche: Im ersten steigt der effektive Zugewinn gegenüber dem versicherungslosen Zustand nurmehr stark verlangsamt an. Spiegelbildlich zur steigenden Selbstbeteiligung sinkt die tatsächlich gewährte Leistung. Da im Bereich steigenden Selbstbehalts das zusätzliche Einkommen 
nicht wie im Fall der Mindestsicherung restlos konfisziert wird ${ }^{97}$, bleibt es allerdings dabei, daß per saldo der effektive Zugewinn aus der Versicherung mit dem Einkommen wächst. Ab dort, wo der maximale Eigenbeitrag erreicht wird (dies ist derzeit bei einem Alterseinkommen von rd. $2.800 \mathrm{hfl}$ der Fall) ${ }^{98}$, verläuft der Anstieg dann ebenso steil wie im Ausgangsfall ohne Selbstbeteiligung, bis die maximale effektive Versicherungsleistung erreicht wird. Diese bestimmt sich nunmehr als Differenz zwischen dem Pflegesatz und der größtmöglichen Selbtbeteiligung.

Von daher enthält der proportionale Beitragstarif gegenüber einer an der tatsächlichen Netto-Leistungserwartung orientierten und deswegen im Sinne einer Pareto-Verbesserung idealen Struktur ein Umverteilungselement zu Lasten der Bezieher von mittleren Einkommen, denen ein Alterseinkommen von weniger als $3.000 \mathrm{hfl}$ entspricht. ${ }^{99}$ Diese "Mittelschicht" aber ist in etwa die Gruppe, von der gezeigt worden ist, daß sie aufgrund der Mehrstufigkeit des Risikos relativ stark von der Pflegeversicherung profitiert.

Die variable Selbstbeteiligung und die präzisere Abbildung der Risikostruktur stellen also zwei gegenläufige Störungen des einfachen Modellfalls dar und heben sich der Tendenz nach auf. Inwieweit allerdings von einer tatsächlichen Kompensation die Rede sein kann, bleibt ungewiß. Um dies zu prüfen, wäre als erstes ein genaues Bild der wirklichen Risiko- und Kostenstruktur im Pflegebereich erforderlich, wie es sich allenfalls unter größtem empirischen Aufwand erstellen ließe. Im Endeffekt bleibt es somit bei der - zunehmend vorsichtiger zu formulierenden - Feststellung, daß die Proportionalität der Beitragslast einer eindeutigen Pareto-Verbesserung gegenüber der versicherungslosen Lage mehr oder weniger nahekommt. Problemlos aufrechterhalten werden kann zumindest die These, daß das praktizierte Verfahren unter dem Gesichtspunkt der angestrebten Pareto-Verbesserung einer einkommensunabhängigen Gestaltung überlegen ist. Eine solche käme der völligen Ausblendung des Einflusses gleich, den die Existenz der Mindestsicherung auf den effektiven Nutzen der

\footnotetext{
${ }^{97}$ sondern nach derzeit geltender Regel zu 87,5\%

${ }^{98}$ Bei einem Alterseinkommen von (maximal) 1.270 im Monat wird der "Standardbeitrag" von 859 hfl fällig. Da vom darüber hinausgehenden Einkommen 87,5\% eingezogen werden, ist der Höchstsatz der Selbstbeteiligung von $2.200 \mathrm{hfl}$ bei $1.270+(2.200-859): 0,875=2.803 \mathrm{hfl}$ erreicht.

${ }^{99}$ Bei kleinen Einkommen fällt eine echte Selbstbeteiligung nicht an, da der fällige Beitrag zu den Pflegekosten nicht höher ist als die häusliche Ersparnis.
} 
Versicherung hat und würde so eine systematische Benachteiligung ärmerer Beitragszahler hervorrufen.

Die Mühen der Ebene, auf der Modell und Wirklichkeit zusammentreffen, sind damit aber noch nicht ausgestanden. Vielmehr sind als nächstes die in den Niederlanden gültigen Beitragsbemessungsgrenzen daraufhin zu prüfen, ob sie mit den im Modell gewonnenen übereinstimmen. Präzise Ergebnisse sind leider auch hier nicht zu erwarten, da das staatliche Rentensystem in den Niederlanden nur eine vom vormaligen Einkommen unabhängige Grundrente gewährt. Daher ist das Verhältnis zwischen Altersund Aktiveinkommen - die Größe r - maßgeblich von der betrieblichen und privaten Altersvorsorge im Einzelfall bestimmt und dürfte stark streuen.

Dennoch läßt sich auf den ersten Blick feststellen, daß beide Grenzwerte gemessen an den Forderungen des Modells bei weitem zu niedrig liegen. Der Freibetrag lag 1990 bei $375 \mathrm{hfl}$ im Monat und damit weit unterhalb des Existenzminimums gilt. Er müßte aber im Umfang des relativen Unterschieds zwischen Alters- und Aktiveinkommen darüber liegen. Ebenso wäre die obere Beitragsbemessungsgrenze dem Modell zufolge oberhalb des stationären Pflegesatzes anzusiedeln, der bei rd. $7.350 \mathrm{hfl}$ pro Monat liegt. ${ }^{100}$ In Wahrheit liegt sie mit rd. $3.300 \mathrm{hfl}$ um mehr als die Hälfte darunter.

Angesichts dieser Diskrepanz erledigt sich gewissermaßen ein Teil der oben zum Tarifverlauf angestellten Überlegungen: Die aufgrund der Selbstbeteiligung markierte Knickstelle - ein Alterseinkommen von $2.800 \mathrm{hfl}$ - liegt offenbar bereits außerhalb der Proportionalzone des tatsächlichen Beitragstarifs, die bei einem beitragspflichtigen Einkommen von $3.300 \mathrm{hfl}$ endet. Somit bleibt der Beitrag bereits in der Zone absolut konstant, die durch den deutlichsten Anstieg des effektiven Versicherungsnutzens gekennzeichnet ist. Dadurch wird der Beitragssatz und damit die Belastung unterer Einkommensgruppen gegenüber einer pareto-optimalen Gestaltung im erläuterten Sinn stark in die Höhe getrieben.

Um diesen Befund richtig einordnen zu können, muß freilich an die Gesamtstruktur der einkommensbezogenen Abgabenbelastung in den Niederlanden erinnert werden. ${ }^{101}$

100 Stichting Centraal Administratie Kantoor AWBZ (1993), S. 55

${ }^{10 !}$ vgl. zum folgenden M.C.F. Meijer et al. (1989) und Periodiek voor Sociale Verzekering (1990) 
Wie schon in groben Zügen dargestellt, existieren drei Tarifstufen, von denen die unterste neben einem geringen Steuersatz alle Volksversicherungsprämien umfaßt. Für Einkommensteile, die die Grenze zur zweiten Stufe überschreiten, werden ausschließlich Steuern gezahlt. Die auf dieser und der dritten Stufe geltenden Sätze sind mit $50 \%$ bzw. $60 \%$ so hoch, daß ein progressiver Verlauf der Gesamtbelastung gewährleistet bleibt.

Es läßt sich mithin die Ansicht vertreten, daß die Pflegeversicherung in einem "ganzheitlichen" Umverteilungsprogramm quasi aufgehe und ein Untersuchungsansatz wie der oben verfolgte deswegen nicht adäquat sei. Für den Einführungszeitpunkt des integrierten Steuer- und Beitrags-Systems erscheint dies auch überzeugend, sofern angenommen werden kann, daß bei der Tarifgestaltung die steuerliche Komponente dazu benutzt worden ist, um bei gegebenen Sozialbeitragslasten ein politisch gewünschtes Tarifprofil der Gesamtbelastung zu erzeugen. Wäre unter diesen Vorzeichen eine höhere Beitragsbemessungsgrenze für die Pflegeversicherung gewählt worden, so hätte ihre Wirkung durch eine gegenläufige Variation der Steuersätze gerade kompensiert werden müssen und können. Im Effekt hätte sich nichts geändert.

Allerdings erscheint es zweifelhaft, ob dem Gesetzgeber ein derart streng zielbewußtes Vorgehen unterstellt werden kann. Im übrigen ist es generell problematisch, Steuern und Sozialversicherungsbeiträge in dieser Weise in einen Topf zu werfen. Dies auch deswegen, weil die hier durchgespielte Sicht für die Zeit nach dem Integrationsakt offenkundig unhaltbar ist: So ist zum Beispiel mit der Erweiterung des AWBZ um den Bereich der Arzneimittelversorgung die Prämie um eineinhalb Prozentpunkte gestiegen, ohne daß dies eine Korrektur der Steuersätze nach sich gezogen hätte. ${ }^{102}$ Dieser Vorgang zeigt, daß die Volksversicherungen eben nicht so fest in das Gesamtsystem eingebettet sind, als daß ihnen keine eigene Verteilungswirkung mehr zugesprochen werden könnte.

Insofern ändert die Nähe zum Steuersystem letztlich nichts an der Forderung, sich auch bei der Fixierung der Beitragsbemessungsgrenze an der Pareto-Bedingung zu

${ }^{102}$ vgl. o.V. (1992a), S. 6 
orientieren. Eine deutliche Anhebung der Grenze erscheint für den Bereich der Pflegeversicherung wünschenswert. ${ }^{103}$

Umsetzungsprobleme ergäben sich freilich auf institutioneller Ebene, da die für den Pflegesektor zuständige Versicherung auch verschiedene andere Schutzfunktionen abdeckt bzw. in Zukunft abdecken soll, aus deren Analyse sich vermutlich auch verschiedene Bemessungsgrenzen (und/oder Tarifstrukturen) ergäben. Solange es bei dieser organisatorischen Zusammenfassung bleibt, was aus Gründen der Kohärenz, Übersichtlichkeit und Verwaltungseffizienz des Systems im übrigen sinnvoll sein kann, dürfte eine modellgerechte Beitragsstruktur sich kaum ableiten und beherrschen lassen. Schon bei der vergleichsweise handlichen Untersuchung der Pflegefunktion allein sind die kaum überwindlichen Grenzen und Unschärfen eines solchen Unternehmens hinreichend deutlich geworden.

\subsubsection{Moral hazard}

\subsubsection{Vorüberlegungen}

Als weiterer Schwerpunkt der Effizienzuntersuchung ist bei der Erarbeitung der Kriterien der Komplex des "Moral hazard" herausgestellt worden. Im Mittelpunkt stand dabei die Befürchtung, daß das Wissen um den Schutz vor finanziellen Folgen die Entscheidung über den Eintritt ins Pflegeheim verzerrt. Allerdings hat die nähere Durchleuchtung dieser und anderer denkbarer Spielarten des Moral hazard zu der These geführt, daß effizienzschädliche Effekte in diesem Sinn als Folge der Einführung einer Pflegeversicherung allenfalls in geringem Umfang zu erwarten seien.

Nunmehr soll versucht werden, diese teilweise auf Plausibilitätserwägungen gestützte These soweit wie möglich anhand der Erfahrungen zu prüfen, die mit dem niederländischen Pflegesicherungskonzept gemacht worden sind. Eine präzise und direkte Messung der Moral hazard-Effekte ist allerdings deswegen unmöglich, weil es dazu

\footnotetext{
${ }^{103}$ Damit wären auch Spannungen im Tarifgefüge entschärft, die sich im Fall steigender Belastung der unteren Tarifstufe mit Volksversicherungsbeiträgen ergeben (vgl. ebenda). Eine mit diesem Problem befaßte Arbeitsgruppe des Finanzministeriums regt in der Tat als eine Lösungsvariante eine Ausdehnung der Beitragspflicht auf die zweite Stufe der Einkommensteuer an (siehe Ministerie van Financien (1993), S. 57), was in etwa einer Verdoppelung der Beitragsbemessungsgrenze gleichkäme (PS (1990), S. 17). Ob dieser Weg beschritten wird, ist allerdings völlig offen.
} 
eines idealen, Fehlanreize ausschließenden, aber versicherten Zustands als Referenzpunkt bedürfte. Es ist betont worden, daß die versicherungslose Lage nicht als Maßstab taugt. Nebenbei ist auch eine solche im strengen Sinn in der Realität nicht auffindbar, da die Mindestsicherung stets als rudimentäre Versicherung wirkt und deshalb ihrerseits nicht frei von Moral hazard-Gefahren ist.

Es ist daher kein theoretisch sauber fundiertes Verfahren, sondern eine grobe, pragmatisch motivierte ${ }^{104}$ Abschätzung, wenn die heutige Heimquote in den Niederlanden mit solchen Werten verglichen wird, die sich ohne gezielte Pflegeversicherung eingestellt haben.

Im übrigen ist ein solches Vorgehen freilich stets mit der Gefahr behaftet, daß die Vergleichswerte von ganz anderen Faktoren als der Sicherungslage einseitig beeinflußt und daher mehr oder weniger untauglich sind. Dies gilt insbesondere dann, wenn ein solcher Vergleich unter Rückgriff auf Zahlen erfolgt, die in den Niederlanden vor 1967 erhoben worden sind. Dieser Ansatz erfreut sich in der Rezeption der niederländischen Erfahrungen durch deutsche Autoren traditionell einiger Beliebtheit. Mit ihm läßt sich in der Tat eine explosionsartige Expansion der Heimpflege aufzeigen, die dann kurzerhand als unerwünschter Versicherungseffekt deklariert wird. ${ }^{105}$

Sinnvoll ist ein solcher Vergleich aber deswegen nicht, weil sich die damals herrschenden Angebots- und Nachfragebedingungen fundamental von den heutigen unterscheiden. Dies betrifft auf seiten des Bedarfs sowohl die sozialen Strukturen als auch das Alters- und Morbiditätsprofil der Bevölkerung, das sich mit dem medizinischen Fortschritt der letzten Jahrzehnte deutlich gewandelt hat. Dem deswegen in den 60er Jahren objektiv geringeren und überdies wohl noch kaum artikulierten Bedarf stand ein entsprechend schwach entwickeltes Angebot gegenüber: Explizit auf die Altenpflege ausgerichtete Heime fingen gerade erst an, sich in nennenswertem Umfang zu entwickeln. ${ }^{106}$

\footnotetext{
${ }^{104}$ Schließlich steht zumindest in der politischen Alltagsdebatte zumeist nicht der "moral hazard" im korrekt abgegrenzten Sinn im Vordergrund, sondern eher die Furcht vor nicht näher differenzierter Nachfrageausweitung als Folge einer Versicherung.

105 siehe beispielsweise W. Keß (1980)

106 Siehe zur Geschichte der Pflegeheime P.J. Blommestijn (1990), S. 132f.: Traditionell wurden gebrechliche alte Menschen, für die nicht (mehr) in der Familie gesorgt werden konnte, in "Armenhäusern, Irrenhäusern und Hospitälern " untergebracht. Das erste Heim für "andauernd kranke Erwachsene"
} 
Es ist daher anzunehmen, daß zumindest ein wesentlicher Teil des Auf- und Ausbaus stationärer Einrichtungen auch ohne die Einführung der Pflegeversicherung erfolgt wäre. Anhand zweier Indizien läßt sich diese Vermutung erhärten:

- Erstens war in den Niederlanden schon in den letzten versicherungslosen Jahren ein rascher Anstieg der Bettenzahl zu verzeichnen, der - in jährlichen Wachstumsraten gemessen - den der Folgejahre sogar übertraf.

- Zweitens hat in der Bundesrepublik Deutschland auch ohne Versicherung eine "vergleichbare Entwicklung stattgefunden"107, was die stationäre Pflegekapazität betrifft. ${ }^{108}$

Was somit als indirektes Prüfverfahren übrigbleibt, ist wohl nur ein Vergleich der niederländischen Daten mit denen anderer Länder ohne Pflegeversicherung. Dabei soll in erster Linie auf die Bundesrepublik (in den Grenzen vor 1990) bezug genommen werden, die aufgrund der soziokulturellen Nähe zu den Niederlanden und des ähnlichen wirtschaftlichen Entwicklungsstandes besonders geeignet erscheint. Zudem ist Deutschland im Unterschied zu beinahe allen übrigen westeuropäischen Ländern ${ }^{109}$ dadurch gekennzeichnet, daß es hier außer der unspezifischen Sozialhilfe fast keinerlei sozialstaatliche Vorrichtungen gibt, die die Funktionen einer Pflegeversicherung für den stationären Bereich zumindest teilweise erfüllen würden. ${ }^{110}$

Eine zusätzliche Schwierigkeit des Vergleichs liegt darin, daß die Anzahl der belegten Pflegeplätze in den Niederlanden aufgrund der beschriebenen restriktiven Kapazitätskon-

wurde erst 1930 eröffnet. Noch 1961 gab es bei einer Bevölkerung von weit über 10 Millionen gerade einmal 6.400 Pflegeplätze.

107 J. Grönert (1981), S. 108

108 Völlig verfehlt ist es deswegen, das prozentuale Kosten- und Mengenwachstum, wie es in den Niederlanden nach $1968 \mathrm{zu}$ beobachten war, ohne Ansehen der völlig unterschiedlichen Ausgangsniveaus zur Schätzgrundlage für die Folgen einer Versicherung zu machen, die Jahrzehnte später in Deutschland eingeführt würde. Auf diesem Weg aber gelingt es zum Beispiel H. Schülke (1984), für die Bundesrepublik ein Kostenvolumen von jährlich 140 Mrd. DM (!) vorherzusagen, das sich nach 12 Jahren als Folge einer Pflegeversicherung einstellen würde. D. Poske (1984), S. 298, hat dieses Verfahren in seiner Kritik an W. Keß (1980) ebenso polemisch wie zutreffend als "tendenziöse Abschreckungspolitik von zweifelhaftem Informationswert" eingestuft.

${ }^{109}$ Zur Situation in europäischen Drittländern siehe etwa K. Großjohann/D. Zöllner(1984) oder aktueller M. Gabanyi et al. (1992).

${ }^{110}$ Ausnahmen sind die Versorgung pflegebedürftiger Beamter und Unfallopfer. 
trolle nicht als getreues Abbild der Entscheidungen Betroffener gewertet werden kann. Es ist vielmehr zum Zweck der Evaluierung von Anreizeffekten erforderlich, den tatsächlich in Heimen Gepflegten diejenigen Personen hinzuzurechnen, die allein wegen des Mangels an Plätzen nicht stationär untergebracht sind. Da aber über die Länge der Wartelisten nur regionale oder aus diesen hochgerechnete Daten vorliegen, leidet die Genauigkeit der Angaben unter einer solchen Korrektur. Erschwerend kommt hinzu, daß Wartelisten auch in deutschen Pflegeheimen üblich sind bzw. waren, ohne daß mir auch nur geschätzte Angaben über deren gesamtes Ausmaß bekannt geworden wären.

Es sollen daher zunächst - im Bewußtsein ihrer Unzulänglichkeit - die faktischen Heimquoten einander gegenübergestellt werden, um danach auf den Korrekturbedarf zurückzukommen. Sie beziehen sich auf 1989 als das letzte Jahr, für das beiderseits umfassende Daten verfügbar sind.

\subsubsection{Ein Heimquotenvergleich}

Als Bezugsgröße zur Messung der Versorgung eines Landes mit Heimpflegeplätzen ${ }^{111}$ erscheint die Bevölkerung im Alter von über 65 Jahren adäquat. In Westdeutschland werden $88 \%$, in den Niederlanden sogar über $91 \%$ der Pflegeplätze von Angehörigen dieser Altersgruppe eingenommen. ${ }^{12}$ Weiterhin erscheint es geboten, innerhalb der so abgegrenzten Altersbevölkerung den Anteil der Hochbetagten zu berücksichtigen: Auf sie entfallen in beiden Ländern über $60 \%$ der Plätze. ${ }^{113}$

Was die Zahl der besetzten Pflegeplätze betrifft, so unterscheiden sich Deutschland und die Niederlande zunächst in Hinblick auf die Verfügbarkeit verläßlicher Daten. Während in den Niederlanden spätestens seit Mitte der 60er Jahre eine genaue Statistik geführt und jährlich aktualisiert wird, hat in Deutschland bis in die jüngste Ver-

\footnotetext{
III Zur sprachlichen Auflockerung ist im folgenden gelegentlich verkürzt von "(Pflege-)Plätzen" die Rede, wenn streng genommen die jeweils besetzten Plätze gemeint sind. Der zahlenmäßige Unterschied ist aufgrund der durchweg sehr hohen Auslastung der Heime im übrigen gering.

${ }^{112}$ Beide Angaben beziehen sich auf das Jahr 1989 (siehe Krug/Reh (1992), S. 27 bzw. NZI (1990), S. 45).

113 jeweils ebenda 
gangenheit eine bemerkenswerte Unsicherheit ${ }^{114}$ über den Gesamtumfang der stationären Pflege geherrscht und zu gravierenden Fehleinschätzungen auch in Hinblick auf die Situation in den Niederlanden geführt. ${ }^{115}$

Als Beispiel hierfür kann die Studie des Kuratoriums Deutsche Altershilfe von 1980 dienen, derzufolge die Heimpflegequote in den Niederlanden seinerzeit fast dreimal so hoch gewesen sein soll wie in der Bundesrepublik. ${ }^{116}$ Diese Angabe stützt sich allein auf bayerische Landesstatistiken. Es kann aber kaum überraschen, daß dieses Bundesland aufgrund seiner ländlich-traditionalen Struktur als pars pro toto denkbar ungeeignet ist. Inzwischen weiß man, daß die Pflegequote in Bayern die bundesweit niedrigste und nur etwa halb so hoch ist wie im Durchschnitt der alten Bundesländer. ${ }^{117}$ Demnach ist zu vermuten, daß schon zum damaligen Erhebungszeitpunkt der Vorsprung der Niederlande nicht 200, sondern vielleicht $50 \%$ betragen hat.

Eine Zählung der Plätze bzw. Patienten in deutschen Pflegeeinrichtungen ist bis heute nicht erfolgt, wohl aber liegt mit der Studie von KRUG und REH nunmehr eine vergleichsweise gut fundierte Schätzung vor, die auf einer Umfrage bei den Heimen selbst basiert. Die Gesamtzahl der belegten Pflegeplätze ist dort für jedes der westdeutschen Bundesländer einzeln wie folgt ermittelt worden: Der vom Statistischen Bundesamt publizierten Zahl der Empfänger von Sozialhilfe zur Pflege in Heimen ist jeweils der landesspezifische Anteil von Selbstzahlern hinzugerechnet worden, wie er sich aus der Befragung der Heime ergab. ${ }^{118}$

Zwar gibt die gerade in bezug auf die Selbstzahler niedrige Rücklaufquote der Fragebogenaktion ${ }^{119}$ Anlaß zur Skepsis; die von KRUG/REH selbst vorgenommene Gegenüberstellung der Resultate mit denen anderweitiger Studien und Schätzungen

\footnotetext{
${ }^{114}$ vgl. H. Braun (1987), S. 13. Die Unzulänglichkeit der deutschen Sozialstatistik insgesamt wird von denen, die sie - etwa als Grundlage politischer Entscheidungen - benutzen müssen, häufig beklagt. Die Sozialexpertin der F.D.P., Frau Dr. BABEL, hat dies mir gegenüber sinngemäß auf die Formel gebracht: "Im Grunde wissen wir gar nicht, wovon wir reden."

${ }^{115}$ Eine irrige Ansicht vom Verhältnis der Heimquoten zwischen Deutschland und den Niederlanden findet sich übrigens auch bei S. Winters (1992), S. 11.

${ }^{116}$ siehe M. v.Rötel et al. (1980), S. 3

${ }^{117}$ W. Krug/G. Reh (1992), S. 27

118 siehe ebenda, S. 24

119 siehe ebenda, S. 17
} 
zeigt jedoch für sechs der elf Bundesländer eine weitgehende Übereinstimmung. In vier der fünf übrigen Länder liegen die externen Angaben zur Selbstzahlerquote mehr oder weniger deutlich über den von KRUG/REH ermittelten Werten ${ }^{120}$, bei denen somit per saldo allenfalls eine leichte Unterzeichnung der Gesamtzahl an Pflegeheimbewohnern zu vermuten ist. Diese wird auf knapp 361.500 beziffert. Wie präzise sie ist, bleibt letztlich dahingestellt; bessere oder auch nur ähnlich vertrauenswürdige Schätzungen existieren derzeit offenbar nicht. ${ }^{121}$

Auf Basis der besprochenen Studie sowie der offenbar lückenlosen Daten für die Niederlande ergibt der Quotenvergleich ein deutliches Bild: Die Heimpflegequote in der üblichen Definition (Quote I) liegt in den Niederlanden um $43 \%$ unter der westdeutschen. Trägt man der hierzulande etwas ungünstigeren Altersstruktur der über 65jährigen dadurch Rechnung, daß man die Platzzahlen allein zur Anzahl der Hochbetagten in Beziehung setzt (Quote II), so bleibt immer noch eine Diskrepanz von rd. 29\%. Wird schließlich ein gewichteter Nenner verwendet, in den die über 80jährigen ihrem Anteil an der Heimbevölkerung gemäß doppelt eingehen, so beläuft sich die Differenz auf genau $40 \%$.

Unterzeichnet wird die Differenz zwischen den Heimquoten möglicherweise dadurch, daß die niederländischen Pflegeheime im Unterschied zu den deutschen auch Aufgaben auf dem Feld der vorübergehenden Kliniknachsorge wahrnehmen. Dies zeigt sich zum einen in der recht großen Zahl von Personen, die aus Pflegeheimen nach Hause entlassen werden. ${ }^{122}$ Zum anderen kommt die unterschiedliche Aufgabenverteilung zwischen Akutkrankenhaus und Pflegeheim wohl auch darin zum Ausdruck, daß die Verweildauer in der Klinik in den Niederlanden für alle Fallkategorien deutlich kürzer ist als in der Bundesrepublik. ${ }^{123}$

\footnotetext{
${ }^{120}$ Errechnet aus Angaben ebenda, Seiten 26 und 13. Siehe auch die dort angegebene Literatur.

${ }^{121}$ Die Bundesregierung nennt 1984 einen Schätzwert von 260.000 stationär Versorgten, der mit dem oben genannten nicht recht vereinbar erscheint: In nur fünf Jahren müßte die Zahl um fast $40 \%$ gestiegen sein. Die Bundesregierung weist aber ausdrücklich darauf hin, daß ihrer Angabe "keine umfassende Erhebung", sondern ein Bündel verschiedenartiger und unvollständiger Daten zugrundeliegt (siehe Deutscher Bundestag (1984), S. 6). Heute wird seitens des BMA ein - wiederum geschätzer - Wert von 450.000 Menschen in Pflegeheimen genannt, der sich auf alle Bundesländer bezieht (BMA (1993), S. 8).

${ }^{12}$ Lt. L.M. Spaan (1989), S.52 war dies bei insgesamt knapp 50.000 Heimplätzen im Jahre 1987 über $8.300 \mathrm{mal}$ der Fall.

${ }^{123}$ siehe OECD (1990), S.164
} 


\section{Tabelle 6.2}

Heimpflegequoten in den Niederlanden und in Westdeutschland 1989

\begin{tabular}{|c|c|c|}
\hline & Niederlande & Deutschland \\
\hline 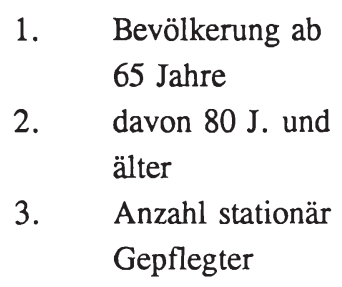 & $\begin{array}{r}1.905 .624 \\
427.715 \\
50.066\end{array}$ & $\begin{array}{r}9.614 .429 \\
2.392 .300 \\
361.489\end{array}$ \\
\hline $\begin{array}{l}\text { Quote I (Zeile 3/ } \\
\text { Zeile 1) } \\
\text { Quote II (Zeile 3/ } \\
\text { Zeile 2) } \\
\text { Quote III (Zeile 3/ } \\
\text { [Zeile 2 + Zeile 1]) }\end{array}$ & $\begin{array}{l}2,63 \\
11,71 \\
2,15\end{array}$ & $\begin{array}{l}3,76^{124} \\
15,11 \\
3,01\end{array}$ \\
\hline
\end{tabular}

Quellen: $\quad$ Krug/Reh (1992), S. 27; Stat. Jahrbuch 1991, S. 64ff. NZI (1990), S. 41f. und CBS (1991), S. 37, eigene Berechnungen

Schließlich ist zu berücksichtigen, daß die Pflegequalität wie gesehen in niederländischen Heimen offenbar höher ist als in deutschen. Es ist anzunehmen, daß hierzulande eine Heimeinweisung auch bei klarer Bedarfslage mitunter "um jeden Preis" vermieden wird. Auch das ließe c.p. eine höhere Heimquote in den Niederlanden erwarten, ohne einen Einfluß des Moral hazard unterstellen zu müssen.

Während sich aus diesen Überlegungen per saldo kein Grund ergibt, eine Überzeichnung der Heimquoten-Differenz anzunehmen, bedarf der Aspekt des für die niederländischen Heime aufgezeigten Nachfrageüberhangs einer gesonderten Überprüfung.

\footnotetext{
${ }^{124} \mathrm{Krug} /$ Reh (1992), S. 27, nennen im Text eine Quote von 4\%, was nur auf grobe Rundung oder auf die Verwendung veralteter Bevölkerungszahlen zurückzuführen sein kann.
} 
Die niederländischen Quoten müssen aufgrund der Rationierung des Angebots faktisch als gedeckelt gelten. Es gilt also eine Schätzung über die Anzahl derjenigen anzustellen, die sich für die Heimpflege entschieden haben, ohne als Inhaber eines Pflegeplatzes in Erscheinung zu treten. Es sind dies die auf den bereits angesprochenen Wartelisten Geführten.

Daten über die Zahl dieser Personen sind mir nur für die Gesundheitsregion Den Haag bekannt geworden. Im Jahre 1988 umfaßte die Warteliste dort insgesamt etwa 500 Personen. ${ }^{125}$ Die Zahl der verfügbaren Betten belief sich auf ungefähr 3.900. ${ }^{126}$ In dieser Region wäre demnach eine Aufstockung der Kapazität um knapp 13\% vonnöten gewesen, um die gesamte Nachfrage zu decken. Laut SPAAN ist die Provinz Zuid-Holland, zu der Den Haag gehört, durch eine stark überdurchschnittliche mittlere Wartezeit gekennzeichnet. ${ }^{127}$ Eine enge Korrelation zwischen Wartezeit und Listenlänge ist anzunehmen.

Es dürfte daher eher einer Überzeichnung der Wartelisten gleichkommen, wenn deren regionaler Umfang als repräsentativ für die Niederlande insgesamt unterstellt wird. Die in der Tabelle angeführte tatsächliche Gesamtzahl belegter Pflegeplätze ist demnach um $13 \%$ zu erhöhen, um auch alle Wartenden mit einiger Sicherheit zu umfassen. Die korrigierten Quoten I-III belaufen sich dann auf 3,04 bzw. 13,23 bzw. 2,43 und sind damit in allen Fällen noch immer geringer als die deutschen Vergleichswerte. Prozentual liegen diese jetzt noch um 14 (Quote II) bis 24\% (Quote I) über den niederländischen Werten. Unberücksichtigt - darauf sei nochmals hingewiesen - bleibt bei diesem modifizierten Vergleich der Tatbestand, daß Wartelisten unbekannten Umfangs auch für deutsche Heime geführt werden.

Damit kann als gesichert gelten, daß es infolge der Pflegeversicherung in den Niederlanden im Endeffekt nicht zu einer anreizbedingt "übermäßigen" Inanspruchnahme von stationärer Pflege gekommen ist. Im Gegenteil scheint diese Option dort heute in signifikantem Maße seltener gewählt zu werden als in der versicherungslosen

\footnotetext{
${ }^{125}$ W.P. Elberse (1989), S. 43

126 Errechnet aus Angaben bei J. van der Bent (1989), Seiten 7 und 11.

${ }^{127}$ Siehe Spaan (1989), S. 41: Für psycho-geriatrische Patienten ist die Wartezeit dort mit Abstand am längsten, für somatische leicht unterdurchschnittlich. Da beide Sektoren i.a. etwa den gleichen Umfang haben, erscheint es sinnvoll, beide Zahlen zu addieren, um ein Gesamtmaß zu erhalten. Nach dieser Rechnung liegt die Provinz Zuid-Holland landesweit an der Spitze.
} 
Bundesrepublik. Ergänzend sei auf eine 1984 publizierte Studie verwiesen, deren Autoren die pflegerische Versorgung in verschiedenen europäischen Staaten vergleichend untersucht haben. Ihr zufolge liegt die niederländische Heimquote mit schon damals 2,7 Plätzen pro 100 Einwohner ab 65 Jahren auf dem vierten von sieben Rängen. Geringeren Vergleichswerten in Großbritannien, der Schweiz und Österreich, stehen - teils deutlich - höhere in Dänemark, Schweden und Norwegen gegenüber. ${ }^{128}$

Wenn in der Literatur gelegentlich weit höhere Quoten stationärer Pflege für die Niederlande angegeben werden ${ }^{129}$, so beruht das auf der Einbeziehung derjenigen Personen, die in Altenheimen außerhalb des Pflegesektors leben. Deren Anteil ist im internationalen Vergleich offenbar außerordentlich hoch. Ein unterschiedsloses Zusammenzählen beider Gruppen ist aber zunächst deswegen sehr problematisch, weil Wohn- und Pflegeeinrichtungen grundsätzlich auf unterschiedliche Bedarfskategorien zielen $^{130}$ und demgemäß ganz unterschiedliche Leistungen bieten. ${ }^{131}$ Auch in Deutschland wird eine entsprechende Trennung vorgenommen und ist für die oben genannten Zahlen berücksichtigt worden.

Dies schließt allerdings noch nicht aus, daß die Grenzlinie von Land zu Land unterschiedlich verläuft und im übrigen in der Praxis verschwimmt. Da - anders als in Deutschland - in den Niederlanden eine klare räumliche und institutionelle Trennung zwischen Pflege- und Wohnheimen besteht, kann vermutet werden, daß in dortigen Altenheimen auch Pflegebedürftige leben, die in der Bundesrepublik unter gleichen Umständen schon als solche deklariert und innerhalb gemischter Einrichtungen in entsprechende Abteilungen verlegt worden wären. ${ }^{132}$

Die Möglichkeit einer solchen Verzerrung fällt im hier vorgegebenen Untersuchungsrahmen aber deswegen kaum ins Gewicht, weil die Bewohner von Altenheimen gerade nicht von der Pflegeversicherung profitieren. Sie müssen vielmehr unter Einsatz

\footnotetext{
${ }^{128}$ siehe K. Großjohann/D. Zöllner (1984), S. 336

${ }^{129}$ siehe etwa M. Gabanyi et al. (1992), S. 24

130 vgl. Ziekenfondsraad (1987), S. 7

${ }^{131}$ Dies spiegelt sich auch darin wider, daß der durchschnittliche Kostensatz bei den Altenheimen um mehr als die Hälfte unter dem der Pflegeheime liegt (siehe Tweede Kamer (1992), S. 116 und 119).

${ }^{132} \mathrm{Da \beta}$ Pflegebedürftigkeit in niederländischen Altenheimen überhaupt vorkommt, kann als gesichert gelten (vgl. W. Groenen/R. van der Wouden (1991), S. 108).
} 
auch ihres Vermögens solange für die gesamten Heimkosten aufkommen, wie dies möglich ist. Selbstverständlich steht ihnen in Anbetracht dieser vergleichsweise unvorteilhaften Regelung die Möglichkeit offen, bei Vorliegen einer entsprechenden Indikation in ein Pflegeheim überzusiedeln bzw. sich in eine entsprechende Warteliste aufnehmen zu lassen. Sofern sie davon Gebrauch machen, sind sie zumindest in den oben angegebenen korrigierten Heimquoten erfaßt.

Von daher läßt sich die hohe Zahl von Heimbewohnern außerhalb der Pflegeeinrichtungen sogar als zusätzliches Indiz gegen die These von der "Sogwirkung" der Pflegeversicherung interpretieren: ${ }^{133}$ Offenbar sind finanzielle Erwägungen selbst dann zumeist nicht ausschlaggebend, wenn ein Verbleib in der häuslichen Umgebung ohnehin nicht mehr zur Debatte steht.

Hinzuzuzählen wären den Heimpflegeplätzen auf niederländischer Seite allerdings die Fälle, in denen im Rahmen eines neueren Programms "externe Heimpflege" gewährt wird. Sie wird von einem Pflegeheim aus zu Lasten der Versicherung organisiert, findet aber im Altenheim statt, um einen Umzug der Betroffenen unnötig zu machen. Diese Variante, deren Umfang inzwischen auf 6.000 Plätze geschätzt wird, existierte zum Vergleichszeitpunkt aber noch nicht. ${ }^{134}$

Als Ergebnis des Quotenvergleichs ist festzustellen, daß es für den oben aus theoretischer Sicht als zweifelhaft eingestuften Moral hazard im Bereich der stationären Pflege auch in der niederländischen Praxis keinerlei Anzeichen gibt. Umgekehrt scheint es dort gelungen zu sein, mithilfe eines breitgefächerten Angebots häuslicher und teilstationärer Hilfen eine vergleichsweise niedrige Heimquote zu erwirken. Dieser Befund stützt die Vermutung, daß die zunächst unter Bedarfsgesichtspunkten als wünschenswert herausgestellte Schaffung vielfältig differenzierter Hilfsdienste auch eine positive Effizienzwirkung zu entfalten geeignet ist. Durch die Möglichkeit zur "Pflege nach Maß" wird ein unnötiger Leistungsverzehr im Sinne des "market basket moral hazard" verhindert.

\footnotetext{
${ }^{133}$ Dabei ist folgende Einschränkung zu machen: Wer über keinerlei Vermögen verfügt bzw. ein solches bereits restlos zur Deckung der Altenheimkosten verbraucht hat, bevor Pflegebedürftigkeit eintritt, hat - bei gleicher Versorgungsqualität - keinen Grund, auf einen Umzug ins Pflegeheim hinzuwirken, da eine Heranziehung von Angehörigen in keinem Fall vorgesehen ist.
}

${ }^{134}$ siehe Tweede Kamer (1994), S. 95 
An dieser Stelle sei abschließend auf einen bemerkenswerten Zusatzaspekt des ZweiLänder-Vergleichs hingewiesen. Einer Untersuchung im Auftrag der $E^{135}$ zufolge leben in den Niederlanden rd. 38\% der über 65jährigen mit eigenen Kindern zusammen, das ist ein Mehrfaches der deutschen Vergleichsquote. ${ }^{136}$ Zum einen kann dieser Befund die relativ niedrige Zahl der Pflegeheimbewohner in den Niederlanden möglicherweise erklären helfen: Da die Alten dort seltener allein leben, sind ihre Chancen besser, einer Heimeinweisung zu entgehen. Daß sie seltener allein wohnen, spricht zumindest deutlich gegen die in Deuschland verbreitete Befürchtung, durch eine Pflegeversicherung würde der familiäre Zusammenhalt untergraben, da sie eine "Abschiebung" lästiger Elternteile begünstige. ${ }^{137}$ Umgekehrt ist anzunehmen, daß auch im Familienzusammenhang die im Wege der Versicherung bereitgestellten ambulanten Hilfen die Tragfähigkeit des häuslichen Pflege-Arrangements wirksam erhöhen.

Da der Unterschied in der Anbindungsquote alter Menschen aber zu groß ist, um allein denjenigen zwischen den Heimquoten zu reflektieren, liegt sogar eine weitergehende Interpretation nahe: Vielleicht sind Angehörige von vornherein eher zur Bildung einer Haushaltsgemeinschaft bereit, wenn sie davon ausgehen können, mit den Folgen einer vorhandenen oder künftigen Pflegebedürftigkeit nicht alleingelassen zu werden. In Anbetracht des überaus hohen Pflegerisikos der hier betrachteten Hochbetagten erscheint es durchaus plausibel, daß solche Überlegungen eine Rolle spielen, zumal andere Erklärungen für eine derart auffällige Diskrepanz angesichts der sozio-ökonomischen und kulturellen Nähe zwischen beiden Ländern nicht recht erkennbar sind.

\footnotetext{
${ }^{135}$ P. Nijkamp et al. (1989), zitiert bei A. Börsch-Supan (1992), S. 158

${ }^{136}$ Direkt angegeben ist ebenda nur der Anteil der über 80jährigen Deutschen im Mehrgenerationenhaushalt. Er beträgt 13,7\%. Für die Gruppen zwischen 65 und 80 liegen die Werte noch deutlich niedriger.

${ }^{137}$ Oft wird diese These sogar unter ausdrücklicher Berufung auf die Niederlande vorgetragen: Den F.D.P.-Bundestagsabgeordneten CRONENBERG hat ein Besuch in holländischen Heimen "davon überzeugt, daß die Gefahr des Abschiebens, (...) wenn Rechtsansprüche bestehen, in erschreckend hohem Maße gegeben ist" (Förderkreis Bad Nauheimer Gespräche (1985), S. 68f.). Angesichts der genannten Zahlenverhältnisse scheint es sich dabei um eine nicht repräsentative, wenn nicht gar um eine gezielt verzerrte Wahrnehmung zu handeln.
} 


\section{Zum Vergleich: Eine Kritik der künftigen Pflegesozialversicherung in der Bundesrepublik Deutschland}

Seit dem 1. Januar 1995 gibt es auch in Deutschland eine gesetzliche Pflegeversicherung. Die Zustimmung des Bundestages am 22. April und des Bundesrates am 29. April 1994 zum Pflege-Versicherungsgesetz (PflegeVG) markiert das (einstweilige) Ende eines jahrelangen politischen Ringens, das von einer nicht minder lebhaften öffentlichen und wissenschaftlichen Debatte begleitet war. Schon eine grobe Dokumentation dieser Entstehungsgeschichte würde Bände füllen und ist hier nicht vorgesehen. Vielmehr soll es im folgenden allein darum gehen, ihr Resultat unter Verwendung einiger der schon auf die niederländische Pflegeversicherung angewandten Kriterien zu prüfen und dabei punktuell Vergleiche mit dem niederländischen System anzustellen.

Es versteht sich dabei von selbst, daß anhand des bloßen Gesetzestextes nur eine vorläufige Bewertung möglich ist. Da die Leistungsgewährung der neuen Versicherung erst im April 1995, für den stationären Bereich sogar erst im Juli 1996 einsetzt, werden die tatsächlichen Effekte erst in einigen Jahren evaluiert werden können. Auf die niederländischen Erfahrungen gestützte Prognosen werden schon aufgrund der "Zeitverschiebung" zwischen beiden Systemen selbst dort kaum sinnvoll aufgestellt werden können, wo die Regelungen vergleichbar erscheinen. Im übrigen beschränkt sich die Betrachtung ganz überwiegend auf die Pflegesozialversicherung, da spezifische Aspekte der privaten Pflichtversicherung in der bisherigen Analyse anhand des niederländischen Modells nicht vorgekommen sind.

Zur Vermeidung unergiebiger Längen wird darauf verzichtet, den Kriterienkatalog Punkt für Punkt abzuarbeiten. Statt dessen wird zunächst die Leistungsseite konzentriert auf die Aspekte der Bedürftigkeitsdefinition und der Selbstbeteiligung erörtert. Im Anschluß kommen die Verläßlichkeit des Arrangements und weitere Effizienzkriterien zur Sprache.

\subsection{Zur Bewertung unter Zielgesichtspunkten}

Die Leistungsseite der deutschen Pflegeversicherung ist entscheidend geprägt von zweierlei Schwellen, über die im Vorfeld kontrovers diskutiert worden ist: das Mindestmaß an Pflegebedarf, das einen Leistungsanspruch auslöst und die nach Pflegestufen 
gestaffelten Obergrenzen der Leistungshöhe. Beide Arten von Begrenzungen sind wichtig im Hinblick auf die Frage, ob die Schutzzusage der Versicherung problemadäquat ist oder nicht.

Die Leistungen der ersten Stufe werden erst dann gewährt, wenn eine "erhebliche" Pflegebedürftigkeit vorliegt. Diese gilt als gegeben, wenn Betroffene "für wenigstens zwei Verrichtungen (...) mindestens einmal täglich der Hilfe bedürfen" (§ 15(1)). ${ }^{1}$ Das Bestehen eines Hilfebedarfs bei der hauswirtschaftlichen Versorgung wird zusätzlich zur Bedingung gemacht. Die Verrichtungen müssen sich auf die Bereiche der Körperpflege, der Ernährung oder der Mobilität" beziehen (ebd.).

Die Zusatzklausel von den "zwei Verrichtungen" ist erst spät in den Gesetzentwurf aufgenommen worden. Offenbar stellt sie die Antwort auf Befürchtungen der Kassenverbände dar, wonach die Stufe I von unerwartet vielen Betroffenen reklamiert und ein nicht weiter spezifizierter täglicher Hilfebedarf auch "konstruiert" werden könnte. ${ }^{2}$ Die Sozialverbände hingegen haben schon die frühere Fassung der Leistungsschwelle als zu hoch bezeichnet. Hierdurch würden von den Pflegebedürftigen "465.000 Menschen, die ursprünglich einbezogen werden sollten, ausgegrenzt". ${ }^{3}$

In der Tat ist die letztlich beschlossene Bedürftigkeitsschwelle in Hinblick auf das Schutzziel problematisch. Sie ermöglicht das Auftreten von Fällen, in denen erhebliche finanzielle Lasten - nämlich die Kosten des täglichen Einsatzes einer professionellen Pflegekraft - ohne jeden Beitrag der Versicherung bewältigt werden müssen. Verschärfend kommt hinzu, daß die Mindestdauer des für die Auslösung von Stufe I täglich erforderlichen Einsatzes vom BMA gegen den Widerstand der Kassen per Ausführungsverordnung auf 90 Minuten festgesetzt worden ist. ${ }^{4}$

Für Bezieher kleiner Renten dürfte dies vielfach den Eintritt der Sozialhilfe-Abhängigkeit bedeuten. Derart Betroffenen muß die Grenzziehung zudem willkürlich erscheinen, da das Hinzutreten einer "zweiten Verrichtung" keineswegs mit (nen-

\footnotetext{
${ }^{1}$ Die Paragraphenangaben beziehen sich auf das Gesetz zur sozialen Absicherung des Risikos der Pflegebedürftigkeit (Pflege-Versicherungsgesetz - PflegeVG) vom 26. Mai 1994, veröffentlicht im Bundesgesetzblatt Teil I Nr.30/1994 vom 28. Mai 1994

${ }^{2}$ vgl. Spitzenverbände der Krankenkassen (1993), S. 64

${ }^{3}$ VdK (1993), S. 144

${ }^{4}$ siehe Handelsblatt vom 9. November 1994: Kassen folgen Blüm
} 
nenswert) höheren Kosten verbunden sein muß. ${ }^{5}$ Von daher ist auch die Begründung der Bundesregierung, daß "bei geringerem Hilfebedarf (..) die Eigenfinanzierung zumutbar" sei, nicht überzeugend. ${ }^{6}$ Um eine Abgrenzung und eindeutige Zählung der jeweils relevanten Verrichtungen zu ermöglichen, ist in §14(4) eine - offenbar abschließende - Liste aufgenommen. ${ }^{7}$

Sind die genannten Voraussetzungen erfüllt, werden Pflegeleistungen im Wert von maximal DM 750 im Monat gewährt. Die Stufe II mit maximal DM 1.800 ist erreicht, wenn "mindestens dreimal täglich zu verschiedenen Tageszeiten" geholfen werden muß. Noch umfangreichere Leistungen setzen voraus, daß "Hilfebedarf rund um die Uhr" besteht. Dann werden Sachleistungen im Wert von DM 2.800, in besonderen Härtefällen bis DM 3.750 gewährt.

Es ist leicht erkennbar, daß die auf den einzelnen Stufen gewährten Leistungen die jeweils entstehenden Kosten zumindest dann nicht vollständig decken, wenn die Hilfe allein von professionellen Diensten zu erbringen ist. Um einen Eindruck vom Ausmaß der Lücken zu erhalten, kann auf den zur Zeit der Expertenanhörung vorliegenden Gesetzentwurf der Fraktionen von CDU/CSU und F.D.P. zurückgegriffen werden. ${ }^{8}$ Dort war neben den monatlichen Kosten auch die Anzahl der finanzierten Pflegeeinsätze nach oben begrenzt. Dem Verhältnis der Mengen- und Wertgrenzen liegt dort eine

\footnotetext{
${ }^{5}$ Nach dem Wortlaut des Gesetzes lassen sich bizarre Konstellationen denken: Wer etwa zwei Pflegeeinsätze täglich benötigt, die nur einer Verrichtung dienen (etwa der Nahrungsaufnahme) hat keinen Anspruch auf Leistungen, während ein Besuch für zwei Verrichtungen finanziert würde. Daß solche Kasuistik in der Anerkennungspraxis keine Rolle spielt, ist nur zu hoffen.

${ }^{6}$ Presse- und Informationsamt der Bundesregierung (1994), S. 2. Bezeichnenderweise endet der zitierte Satz dort wie folgt: "...oder es kann - bei Bedürftigkeit - Sozialhilfe in Anspruch genommen werden. "

${ }^{7}$ Dennoch bleiben auch diesbezüglich Fragen offen: Ist das dort aufgeführte "An- und Auskleiden" im Sinne der nōtigen Zählung eine Verrichtung oder sind es zwei? - Die Paragraphen 16 und 17 deuten im übrigen darauf hin, daß der Gesetzgeber selbst die hier besprochenen Bestmmungen nicht für praktisch hinreichend hält: Das Bundesarbeitsministerium ist ermächtigt, "Vorschriften zur näheren Abgrenzung" zu erlassen. Von den Spitzenverbänden der Pflegekassen zu beschließende Richtlinien (§ 17) dienen demselben Konkretisierungszweck.
}

${ }^{8}$ siehe Deutscher Bundestag (1993), S. 20 (alter § 33) 
Kostennorm von DM 30,- pro Einsatz ${ }^{9}$ zugrunde, die eher den unteren Rand des Realistischen markieren dürfte. ${ }^{10}$

Auf Stufe I werden demnach bis zu 25 Einsätze im Monat von der Versicherung übernommen. Der minimale Bedarf beträgt hingegen definitionsgemäß $30+x$ Einsätze, wobei das $\mathrm{x}$ für den in dieser Stufe ebenfalls vorausgesetzten hauswirtschaftlichen Bedarf steht, der "mehrfach in der Woche" gegeben sein muß. Werden demgemäß in vorsichtiger Näherung pro Monat 10 solche Einsätze zu einem ermäßigten Kostensatz von DM 20,- unterstellt, so belaufen sich die Gesamtkosten auf DM 1.100. Die Selbstbeteiligung beträgt mithin DM 350 oder knapp $32 \%$.

Bei dieser Rechnung ist aber noch vorausgesetzt, daß mit DM 30 sogar ein 90-MinutenEinsatz bestritten werden könne, wie er für die Anerkennung eines Leistungsanspruchs erforderlich sein muß. Das ist eindeutig unrealistisch. Werden dafür - immer noch vorsichtig - DM 45 angesetzt, so erhöhen sich die monatlichen Gesamtkosten auf DM 1.550 und die Selbstbeteiligung auf über $51 \%$.

Da auch eine Bedarfsfrequenz von zwei Einsätzen ${ }^{11}$ noch unter Stufe I fällt, vergrößert sich in solchen Fällen die Deckungslücke nochmals: Bei unverändertem Bedarf bezüglich der Haushaltsführung ergeben sich Kosten von 60 x DM $30+10$ x DM 20 = DM 2.000, von denen die Versicherung wiederum DM 750 trägt. Der Eigenbeitrag erreicht nun DM 1.250 oder $62,5 \%$.

An der Unterkante von Stufe II stehen reinen Pflegekosten von DM 2.700 für drei Einsätze am Tag Leistungen in Höhe von DM 1.800 gegenüber. Unter Einschluß des hauswirtschaftlichen Bedarfs ${ }^{12}$ beträgt der Eigenanteil des Versicherten an den Kosten dann $38 \%$ oder DM 1.100 .

\footnotetext{
${ }^{9}$ Genau DM 30 pro Einsatz errechnen sich für die Stufen I und II, in der dritten Stufe sind merkwürdigerweise nur DM 28 impliziert.

${ }^{10} \mathrm{~J}$. Allemeyer (1993), S. 812 nennt für die Sozialstationen einen Satz von derzeit DM 41 pro Stunde, wobei die Wegezeiten mitgezählt werden. Etwa 60 Minuten betrage im Durchschnitt aber schon die Netto-Einsatzzeit.

"Denkbar sind auch durchschnittlich 2,5 Einsätze pro Tag. Davon wird hier abgesehen.

${ }^{12}$ Dabei bleibt unberücksichtigt, daß der auf Stufe II vorhandene ausgeprägte Pflegebedarf vielfach auch mit erhöhtem Bedarf im Haushalt einhergehen dürfte.
} 
Analog zu den Überlegungen auf Stufe I sind Fälle vorstellbar, in denen auch drei Besuche täglich nicht ausreichen, ohne daß von einem Bedarf "rund um die Uhr, auch nachts" (§ 15(1)) im Sinne der Stufe III die Rede sein kann. Die vom Betroffenen selbst zu tragenden Kosten betragen bei vier Einsätzen DM $2.000=53 \%$, bei fünf Einsätzen DM $2.900=62 \%$.

Was die dritte Stufe betrifft, so lassen sich die bislang angestellten Berechnungen nur bedingt fortsetzen. Es versteht sich von selbst, daß eine häusliche 24-Stunden Betreuung auf rein professioneller Basis immense Kosten verursacht, da mindestens drei Vollzeitkräfte erforderlich wären. So gesehen deckt die Versicherung in solchen Fällen mit DM 2.800 und selbst im besonderen Härtefall ${ }^{13}$ mit DM 3.750 nur einen kleinen Teil. In Einsätze zum Standardpreis umgerechnet kommt die Pflegekasse für 3,1, im Extremfall für 4,2 Einsätze täglich auf. Die naheliegende Heimeinweisung wird sich also hier nur vermeiden lassen, wenn die Betroffenen selbst in erheblichem Umfang finanzielle und/oder personelle Ressourcen mobilisieren können. Für eine wertende Zusammenfassung in Hinblick auf die Angemessenheit der ambulanten Sachleistungen sind die folgenden Punkte wichtig:

Dort, wo Pflegebedürftige vollständig auf den Erhalt professioneller Dienste angewiesen sind, decken die Leistungen der Versicherung mitunter nicht einmal die Hälfte des Bedarfs. Wird zum einen eine ganze Kategorie von durchaus gravierenden und kostspieligen Fällen von Leistungsbezug vollends ausgeschlossen, so haben zum anderen auch Betroffene mit Anspruch auf Leistungen vielfach mit vierstelligen Beträgen im Monat selbst einzustehen. Beides wird regelmäßig den Eintritt der Sozialhilfe nach sich ziehen. ${ }^{14}$ Zudem erscheint die Formulierung der Eingangsschwelle in Anbetracht bestimmter Fallkonstellationen willkürlich und die recht grobe Einteilung der Stufen führt zu einem sprunghaften Verlauf der Lastenverteilung über das Spektrum der Fälle.

\footnotetext{
${ }^{13}$ Zum Härtefall siehe § 36(4). Die Feststellung eines solchen obliegt den Pflegekassen nach offenbar weitgehend eigenem Ermessen, wobei maximal 3 \% der auf Stufe III eingeordneten von dieser Klausel betroffen sein dürfen. Da aber die Stufe III aufgrund ihrer "anspruchsvollen" Definition nur eher selten auftreten wird, ist die Zahl der Härtefälle eng begrenzt.

${ }^{14}$ Ein Quantifizierungsversuch wäre mit einer Vielzahl von Unwägbarkeiten behaftet und soll hier unterbleiben. Man halte sich aber in Anbetracht der beispielhaft genannten monatlichen Belastungen vor Augen, daß - anders als oftmals im Fall der stationären Pflege - in den hier besprochenen Situationen die Kosten der normalen Lebensführung etwa für Wohnungsmiete und Ernährung weiterhin anfallen. Die nach deren Abzug verbleibende "freie Spitze" des Einkommens aber dürfte bei vielen Renterinnen und Rentnern nicht für die genannten Bedarfsgrößen ausreichen.
} 
Andererseits werden die gebotenen Leistungen dort ausreichend sein, wo pflegende Angehörige oder Hausgenossen verfügbar sind und lediglich der Entlastung bedürfen. Hier lassen sich sogar realistische Fälle denken, in denen die Bemessung der Hilfen recht großzügig erscheint. ${ }^{15}$ Das Problem liegt demnach offenbar darin, daß der Leistungskatalog unzureichend differenziert ist, indem er allein auf das Fähigkeitsprofil des betroffenen Individuums abstellt. Die niederländische Lösung ist in diesem Punkt insofern überlegen, als sie den aus der Lebenssituation bestimmten effektiven Bedarf im Einzelfall zu erfassen versucht. Nur ein solches Vorgehen trägt dem Subsidiaritätsgedanken angemessen Rechnung, wonach die Sozialversicherung nur dann, aber auch immer dann im nötigen Umfang tätig werden muß, wenn eine Problemlösung im privaten Umfeld nicht (mehr) möglich oder zumutbar ist. Ein pauschales Knapphalten der Leistungen, wie es in der starren Systematik der Pflegestufen angelegt ist, führt zu kaum akzeptablen Sicherheitslücken.

Unberührt bleibt von solcher Kritik indes die Feststellung, daß die Bedingungen für die häusliche Pflege sich im Vergleich mit der Ausgangslage vor Einführung der Pflegeversicherung in manchen Fällen deutlich verbessern werden. Bislang nämlich werden - abgesehen von der Sozialhilfe - Hilfen in diesem Bereich allein im Rahmen der Krankenversicherung gewährt. Deren Leistungen sind jedoch sowohl hinsichtlich des begünstigten Personenkreises als auch der Höhe nach noch weitaus restriktiver geregelt als dies für die Pflegeversicherung vorgesehen ist. ${ }^{16}$

Auch im Fall der Heimpflege richten sich die Leistungen der deutschen Pflegeversicherung nicht nach den faktischen Kosten, sondern in der Regel wohl nach dem Höchstbetrag von DM 2.800, wobei aber zusätzlich die Klausel gilt, daß im Durchschnitt nicht mehr als DM 30.000 im Jahr gewährt werden dürfen (§ 43). Die genaue Wirkung dieser Regelung bleibt abzuwarten: Offenbar wird der Höchstsatz dann gesenkt werden müssen, wenn sich nicht genug Fälle einstellen, deren Kosten unterhalb der Durchschnittsgrenze liegen. Solche Fälle wird es wiederum angesichts des heutigen ${ }^{17}$

15 Etwa im Fall eines uneingeschränkt leistungsfähigen Rentners, dessen behinderte Ehefrau der Unterstützung beim Ankleiden und Treppensteigen bedarf: Die Versicherung zahlt 25 Pflegebesuche im Monat.

${ }^{16}$ siehe dazu Deutscher Bundestag (1993), S. 70

${ }^{17}$ Zur Erinnerung: Die Leistungen für stationäre Betreuung werden erst ab 1996 gewährt. Bis dahin dürften die Pflegesătze weiter gestiegen sein. 
Durchschnittspflegesatzes auf Stufe III von etwa DM $4.000^{18}$ nur dann geben, wenn ein hoher Anteil davon auf "Unterkunft und Verpflegung" entfällt. Diese Kostenkomponenten nämlich sind grundsätzlich nicht erstattungsfähig.

So oder so sind auch im Bereich der stationären Versorgung erhebliche Finanzierungslücken unausbleiblich. Die fällige Zuzahlung durch die Betroffenen dürfte durchschnittlich bei annähernd DM 2.000 liegen, mitunter auch weit darüber. Das Ziel der Versicherung, alten Menschen eine durch den Pflegebedarf verursachte Abhängigkeit von der Sozialhilfe zu ersparen, wird in erheblichem Umfang verfehlt. ${ }^{19}$ Dessen ist sich die Bundesregierung auch bewußt, wie einige gewundene Formulierungen zur Erläuterung des Gesetzes erkennen lassen:

"Wer sein Leben lang gearbeitet, Steuern und Beiträge gezahlt und eine durchschnittliche Rente erworben hat, ist im Regelfall künftig in der Lage", ohne Sozialhilfe auszukommen. ${ }^{20}$ Insbesondere für Frauen, die sehr wohl zeitlebens gearbeitet, aber dabei aufgrund von Lücken in der Erwerbsbiographie und/oder niedrigen Einkommen keine durchschnittliche Rente erworben haben, muß dies wie Hohn klingen. Zur Begründung wird dann noch angeführt, die Pflegeversicherung könne schließlich "eine fehlende Rente nicht ersetzen und eine kleine Rente nicht anheben". Diese Aussage ist ebenso zweifelsfrei richtig, wie sie das Thema verfehlt. Durchaus nämlich kann von einer Pflegeversicherung erwartet werden, daß sie auch im Fall kleiner Renten dafür sorgt, daß die aus Pflegebedarf resultierenden Lasten tragbar bleiben.

Der Schlüssel dazu wäre - nach niederländischem Vor-

bild - die Staffelung der Eigenbeteiligung nach dem Einkommen unter Wahrung gewisser Obergrenzen, die der Versicherungscharakter des Systems gebietet. Ebenso wie im Fall der soeben kritisierten Abgrenzung der Bedarfsstufen ist es also auch hier neben dem Wunsch nach strikter Begrenzung der Versicherungskosten - die Starrheit der Regelung, welche die Schutzfunktion der Versicherung empfindlich einschränkt.

\footnotetext{
${ }^{18} \mathrm{Vgl}$. etwa J. Allemeyer (1993), S. 811 in bezug auf die Hamburger Pflegeheime.

${ }^{19}$ Das DIW (1993), S. 422, schätzt den Anteil der Heimbewohner, die trotz Versicherung sozialhilfeabhängig sein werden, auf $40 \%$ in Westdeutschland, während dies im Osten des Landes nach wie vor "fast alle stationär Gepflegten" beträfe. Diese Schätzung beruht zwar noch auf der zeitweise vorgesehenen maximalen Versicherungsleistung von DM 2.100, aber auch auf den Pflegesätzen von 1993, so das per saldo kein großer Korrekturbedarf bestehen dürfte.

${ }^{20}$ Presse- und Informationsamt der Bundesregierung (1994), S. 2 (Hervorhebungen hinzugefügt)
} 
Besonders knapp und zusätzlich an die Erfüllung bestimmter Bedingungen geknüpft sind die Leistungen der Pflegeversicherung dann, wenn sie in Geldform gewählt und gewährt werden. Hier hat sich der Gesetzgeber offensichtlich die oben ausgeführten Bedenken gegen das Instrument des Pflegegeldes in hohem Maße zu eigen gemacht, ohne indes ganz auf eine solche Option verzichten zu wollen: Zum einen ist die Attraktivität der Entscheidung für Geldleistungen dadurch gemindert, daß sie nur etwa halb so hoch sind wie der Wert der jeweils möglichen Sachleistungen. ${ }^{21}$ Zum anderen setzt der Pflegegeldbezug das Vorhandensein einer "Pflegeperson" voraus, deren Eignung in Hinblick auf den jeweiligen Bedarf zudem geprüft und anerkannt worden sein muß (§ 37(1)).

Zusätzlich werden die Empfänger verpflichtet, in gewissen Abständen einen professionellen Einsatz stattfinden zu lassen (§ 37(3)). Die Kosten dieses Kontrollbesuchs sind aus dem Pflegegeld zu bestreiten, welches dadurch einer - wenn auch geringfügigen - partiellen Zweckbindung unterliegt. Es ist im übrigen offensichtlich, daß eine "Entlohnung" der Pflegeperson aus den Unterstützungsgeldern nicht erfolgen kann. Als Pflegeperson gilt nämlich laut $\S 19$ nur, wer mindestens 14 Stunden pro Woche tätig ist. Daraus ergibt sich auf Stufe I im günstigsten Fall ein "Stundenlohn" von DM 400/56 Stunden = DM 7,14. Im Fall der Stufe II könnte sich dieser Betrag zwar theoretisch verdoppeln, dort aber ist angesichts der oben genannten Bedarfsuntergrenze davon auszugehen, daß 14 Wochenstunden privater Pflege und Hauswirtschaftshilfe bei weitem nicht hinreichend sind.

Damit muten die Regelungen zum Pflegegeld halbherzig an. Sind die genannten Prüfund Kontrollklauseln sicherlich sinnvoll, um einer Zweckentfremdung der Zuschüsse zulasten der Versicherung oder auch der Versicherten vorzubeugen, so ist es auf dem Hintergrund dieser Vorkehrungen nicht leicht einzusehen, warum die Gelder - zumal so weit - unterhalb des Sachleistungsniveaus angesetzt worden sind. Schließlich wird dadurch ein evtl. eintretender echter Mißbrauch im Sinne der Erschleichung von Transfers nicht verhindert ${ }^{22}$, sondern nur in seiner Schadenshöhe begrenzt. Weitaus

\footnotetext{
${ }^{21}$ Das exakte Verhältnis variiert dabei von Stufe zu Stufe.

${ }^{2}$ Ein Wahlrecht zwischen DM 400 in bar und Sachleistungen für DM 750 bestand schon vor Einführung der Pflegeversicherung in bezug auf die Pflegeleistungen der Krankenkassen. Wenn dort zu rd. 80\% die Geldleistung gewählt worden ist (siehe Dt. Bundestag (1993), S. 70), so dürften dahinter auch "Mitnahmeeffekte", also solche Fälle verborgen sein, in denen sich die Lage des Pflegebedürftigen selbst durch die Zahlung nicht verändert hat.
} 
klarer als eine auf diesem Weg denkbare Ersparnis ist der negative Effekt der knappen Bemessung: Die formal vorgesehene Wahlmöglichkeit der Betroffenen wird durch sie faktisch stark eingeschränkt. Die Option einer privaten Regelung des Pflegefalls wird nur dort gegeben sein, wo eine hinreichend motivierte Pflegeperson im Haushalt ohnehin verfügbar ist.

Gleichwohl darf es im deutsch-niederländischen Vergleich als Pluspunkt unter dem Aspekt der Flexibilität im Einzelfall angesehen werden, daß überhaupt Geldleistungen vorgesehen sind und zudem - freilich ohne Kumulation - mit Sachleistungen kombiniert werden können ( $\S 38) .{ }^{23}$ In welchem Umfang von diesen Optionen unter den genannten restriktiven Bedingungen Gebrauch gemacht wird, bleibt abzuwarten.

Die möglichen Auswirkungen der deutschen Pflegeversicherung auf die Entwicklung der Leistungsqualität sollen wie bisher nur für den stationären Bereich kurz erörtert werden. Grundsätzlich haben die Pflegekassen nach § 28(3) "sicherzustellen, daß die Leistungen (...) nach allgemein anerkanntem Stand medizinisch-pflegerischer Erkenntnisse erbracht werden." Gleichzeitig dürfen sie "das Maß des Notwendigen nicht übersteigen". ${ }^{24}$ Diese eher abstrakten Vorgaben bedürfen erkennbar der praktischen Ausfüllung. Im hier vorliegenden Zusammenhang läßt sich etwa die Frage aufwerfen, ob mit einer Verbesserung der Personalsituation in Pflegeheimen zu rechnen ist, wie sie in den Niederlanden mit der Konsequenz zu beobachten war, daß dort heute bezogen auf die Zahl der Plätze mehr als doppelt soviel Pflegepersonal zur Verfügung steht wie in Deutschland.

Diese Frage ist klar zu verneinen. Der durch die Pflegesätze bestimmte finanzielle Rahmen, in dem sich die Personalausstattung als zentraler Kostenfaktor bewegt, ist ähnlich wie die Versorgungsverträge über den Inhalt der Pflege nach $\S 72$ - zwischen dem Heimträger auf der einen und den betroffenen Pflegekassen sowie den zuständigen Sozialhilfeträgern auf der anderen Seite auszuhandeln.$^{25}$ Die letzteren verweisen zur Begründung ihres Mitspracherechts zurecht darauf, daß "die Spitzen-

\footnotetext{
${ }^{23}$ In den Niederlanden wäre eine entsprechende Auflockerung des Sachleistungsprinzips übrigens auch in Hinblick auf die Wartelisten wünschenswert. Wenn eine als nötig anerkannte Sachleistung aus Kapazitätsgründen nicht sofort erlangt werden kann, hätte ein ersatzweise gewährtes Pflegegeld den Charakter einer Überbrückungshilfe.
}

${ }^{24} \S 29(1)$

25 siehe $\$ 85$ 
finanzierung letztlich zu Lasten der (...) Sozialhilfe erfolgt ${ }^{26}$. Ebenso wie bisher ${ }^{27}$ werden somit die Agenturen der Mindestsicherung bei der Entscheidung über die Heimausstattung das letzte Wort haben, gerade von ihnen aber ist ein ausgprägtes Interesse an der Verbesserung der Standards naturgemäß nicht zu erwarten. ${ }^{28}$

Aber selbst wenn die Pflegekassen in den Verhandlungen freie Hand hätten und - selbst aufgrund der Leistungsdeckelung weitgehend unberührt von der Höhe der Pflegesätze allein die Interessen ihrer Kunden zu befördern versuchten, wäre ihre Situation zwiespältig: Je höher die als notwendig anerkannten Standards, desto unzureichender ihre Leistungen; die von der Qualitätsverbesserung Begünstigten müßten dafür - oftmals im Wege der Verarmung - selbst bezahlen. Im übrigen gibt auch die Bundesregierung implizit zu erkennen, daß sie eine Qualitätssteigerung im Sinne von mehr Pflegepersonal auch zukünftig nicht für geboten hält. ${ }^{29}$

Insgesamt schneidet das Pflege-Versicherungsgesetz bei der Bewertung anhand der in dieser Arbeit formulierten Zielvorgaben eher schlecht ab. Dies gilt auch im Vergleich zu dem Befund, der im vorangegangenen Kapitel für die Niederlande aufgestellt worden ist. Die gewährten Leistungen sind vielfach unzulänglich und erreichen nur einen Teil der vom Schadensfall Betroffenen überhaupt. So wird weder die Notwendigkeit des Rückgriffs auf Sozialhilfe auch nur einigermaßen konsequent vermieden, noch ist im stationären Bereich mit einer Anhebung der vergleichsweise dürftigen Pflegestandards ${ }^{30} \mathrm{zu}$ rechnen.

\footnotetext{
${ }^{26}$ Bundesvereinigung der kommunalen Spitzenverbände (1993), S. 340

${ }^{27}$ Oder gar mehr als bisher: Da "an der Basis" eine Entlastung der Sozialhilfe erfolgt, verkleinert sich insgesamt der bei ihr anfallende Posten pflegebedingter Aufwendungen. Wenn nun als Zielgröße der Kommunen im Rahmen ihrer Haushaltspolitik eine maximale Steigerungsrate dieser Lasten fungiert, erscheint eine harte Verhandlungsführung sogar noch notwendiger als zuvor: schon eine zehnprozentige Steigerung der Pflegesätze könnte die darin enthaltenen Sozialhilfelasten leicht verdoppeln.

28 vgl. DIW (1993), S. 421

${ }^{29}$ Es wird zur Rechtfertigung der angestrebten Stabilität des Beitragssatzes (siehe nächster Abschnitt) folgende Rechnung aufgemacht: Das Beitragsvolumen steigt "jährlich entsprechend der Lohnentwicklung. Damit können die üblichen Preissteigerungen, die insbesondere aus den Löhnen für das Pflegepersonal herrühren, ausgeglichen werden" (Presse- und Informationsamt (1994), S. 2). Hier wird also - unter anderem - die Personalstärke konstant gesetzt.

${ }^{30}$ Wenn die Schilderungen vom Alltag in Pflegeheimen, wie sie etwa von Vertretern der AWO zu lesen sind, auch nur halbwegs zutreffen, können die Zustände auch mit vollem Recht als "absolut dürftig " bezeichnet werden (siehe Süddeutsche Zeitung vom 18. September 1994: Pflegeheime werden zu
} 
Das in mancher Hinsicht anspruchsvollere Kriterienbündel, wie es sich aus der Einstufung der Pflege als egalitär zuzuweisendes Gut des Grundbedarfs ergibt, braucht unter diesen Umständen gar nicht erst ausführlich angelegt zu werden. Es ist offensichtlich, daß der zur Bedarfsdeckung regelmäßig erforderliche Zukauf von Pflegeoder von Versicherungsleistungen mit einem erheblichen Einfluß der individuellen Kaufkraft auf die Qualität der Sicherung und/oder der Versorgung verbunden ist.

Unter Zielaspekten dem niederländischen Modell überlegen erscheint die deutsche Pflegeversicherung allein in der Möglichkeit, frei verfügbare Geldleistungen zu wählen. Sie birgt unter den dargelegten Einschränkungen fallweise die Chance, auf individuelle Bedarfslagen flexibler $\mathrm{zu}$ reagieren. Weiterhin besteht aufgrund des Sicherstellungsauftrags zumindest die Hoffnung, daß die für die Niederlande als zentraler Mangel beschriebenen Kapazitätsengpässe in Deutschland vermieden werden können. Allerdings sind die Pflegekassen bei der Erfüllung des Auftrags auf die Bereitschaft der Bundesländer angewiesen, in ausreichendem Umfang in den Aufbau von Pflegeeinrichtungen zu investieren. Da den Ländern diese Aufgabe im Pflegegesetz in recht unverbindlicher und unpräziser Form übertragen worden ist (siehe § 9), sehen die Kassen den Folgen dieser Regelung mit Skepsis entgegen. ${ }^{31}$

\subsection{Zur Bewertung unter Effizienzgesichtspunkten}

Ansätze zur Bewertung unter dem Aspekt der Verläßlichkeit als des ersten Effizienzkriteriums ergeben sich bereits aus den bisherigen Kritikpunkten: Der Eindruck, wirklich und wirksam geschützt zu sein, wird sich bei den Versicherten von vornherein nur bedingt einstellen, wenn

- nicht in allen Pflegefällen überhaupt Leistungen gewährt werden,

- gewährte Leistungen nicht in allen Fällen die Beantragung von Sozialhilfe erübrigen und

- die Qualität der Leistungen zweifelhaft erscheint.

\footnotetext{
Sterbeheimen).

${ }^{31}$ siehe Handelsblatt vom 4. Mai 1994: "Zweifel an der Bereitschaft der Länder zum Aufbau der Pflegeinfrastruktur" : Sollten sich diese Zweifel als begründet erweisen, könnten Kapazitätslücken nur dadurch vermieden werden, daß nötige Investitionen zulasten der Pflegesätze vorgenommen würden. Dies würde den Deckungsgrad der Versicherungsleistungen nochmals spürbar absenken.
} 
Zusätzlich aber steht die derart eingeschränkte Schutzzusage unter dem Finanzierungsvorbehalt der "Beitragssatzstabilität", die in $\S 70$ als "Allgemeiner Grundsatz" festgelegt ist. Demnach sind solche Vereinbarungen mit den Anbietern von Pflegeleistungen unwirksam, deren Erfüllung den durch die festgesetzten Beitragssätze gesteckten Rahmen sprengen würde.

Diese Vorschrift erscheint insofern redundant, als die Pflegekassen die jeweils auszuhandelnden Pflegepreise nicht vollständig, sondern nur bis zum jeweiligen Leistungsdeckel selbst bezahlen müssen. Entscheidender für die faktische Einhaltung der Beitragssatzstabilität ist daher der mit "Dynamisierung" betitelte $\S 30$, der die Regierung ermächtigt, "die Höhe der Leistungen im Rahmen des geltenden Beitragssatzes und der sich daraus ergebenden Einnahmenentwicklung anzupassen". ${ }^{32}$ Offenkundig kann hier also auch nach unten "dynamisiert" werden, wenn die Entwicklung des Beitragsaufkommens schwächer verläuft als die der Fallzahlen.

Automatisch erhöht sich der ohnehin hohe Eigenanteil der Pflegebedürftigen an den Kosten im übrigen freilich dann, wenn - bei gegebenen Fallzahlen - die Pflegepreise stärker als die Summe der beitragspflichtigen Einkommen wachsen. Aber auch bei konstantem Eigenanteil können sich mit der Zeit die Finanzierungsbedingungen für Betroffene dadurch verschlechtern, daß die den Nettolöhnen folgende Rentenentwicklung hinter dem vor allem durch die Bruttopersonalkosten bestimmten Verlauf der Pflegesätze zurückbleibt. ${ }^{33}$

Vor allem angesichts der demographischen Entwicklung ist das ohnehin knapp kalkulierte Niveau der Versicherungsleistungen damit ständig von der Aushöhlung bedroht. ${ }^{34}$ Auf die wiederholt vorgetragenen Warnungen vor einem raschen und

\footnotetext{
${ }^{32}$ Hierzu bedarf sie allerdings der Zustimmung des Bundesrats. Was bei Verweigerung dieser Zustimmung geschieht, solange eine Gesetzesänderung zur Erhöhung des Beitragssatzes ebenfalls nicht durchsetzbar ist, bleibt unklar. Dies ist nur ein Beispiel dafür, daß der Zwang zum Kompromiß zwischen völlig konträren Positionen im PflegeVG zu sehr eigenwilligen Regelungen mit "Selbstzerstörungspotential" geführt hat. Bei der zweistufigen "Kompensation durch Feiertagsstreichung" (unter Einschaltung des SVR) ist das schon vor Inkrafttreten des Gesetzes sehr deutlich geworden.

${ }^{33} \mathrm{Vgl}$. W. Schmähl (1993), S. 364. Es kann sich die Nettogröße freilich (theoretisch?) auch günstiger entwickeln als der Bruttolohn.

${ }^{34}$ Es sei nochmals betont, daß ein Anwachsen der Zahl alter Menschen keineswegs zwingend zu einem proportionalen Anstieg der Pflegefälle führt. Überdies kann sich eine Verschiebung der Proportion zwischen stationärer und ambulanter Pflege per saldo kostendämpfend auswirken. Dennoch ist es
} 
unaufhaltsamen Anstieg der relativen Beitragslast hat die Bundesregierung damit reagiert, daß sie eine solche Entwicklung quasi "verbietet" und die dafür ursächliche absolute und relative Verstärkung der Altersbevölkerung einstweilen ignoriert. ${ }^{35}$ Wenn dieser Prozeß dennoch in Gang kommt, werden die Verantwortlichen wiederholt vor der Wahl stehen, den Beitragssatz per Gesetzgebungsverfahren doch zu erhöhen oder dem allmählichen "Absterben" der Pflegeversicherung seinen Lauf zu lassen.

Unter diesen Vorzeichen kann von einer verläßlichen Schutzzusage gegenüber künftigen Generationen von Pflegebedürftigen keine Rede sein. Der Versuch, die Wahl des Umlageverfahrens mit einer Art Garantie stabiler Beitragssätze zu vereinbaren, ist letztlich entweder illusionär oder führt den Versicherungsgedanken ad absurdum. Bei diesem Verdikt ist es wichtig zu betonen, daß die Alternative zum Stabilitätsgebot in der vorliegenden starren Formulierung mitnichten darin gelegen hätte, durch eine völlige Freigabe des Beitragssatzes dessen mitunter befürchtete "Explosion" in Kauf zu nehmen. Eine solche wäre unter den gegebenen Umständen schon deshalb ohnehin nicht zu erwarten gewesen, weil die gedeckelten Leistungen gegen die Preisentwicklung im Pflegesektor weitgehend immun sind.

Somit verbleibt allein die Leistungsmenge, mithin die Fallzahl, von der ein beitragstreibender Effekt hätte ausgehen können. In diese Komponente wiederum gehen neben der absolut und in Relation zur Beitragsbasis wachsenden Altersbevölkerung die altersgruppenspezifischen Pflegequoten ein. Der - angesichts der niederländischen Befunde eher unbegründeten - Furcht vor einem versicherungsbedingten Anstieg dieser Quoten hätte etwa dadurch Rechnung getragen werden können, daß die maximale Steigerung des Beitragssatzes an einen geeigneten, rein demographisch definierten Index gekoppelt worden wäre. ${ }^{36}$

Auf diesem Weg wäre es prinzipiell möglich gewesen, das im Einführungszeitpunkt als minimal notwendig anerkannte Sicherungsniveau auch für die Zukunft zu gewährleisten, ohne sich dabei einer unkontrollierten oder unabsehbaren Entwicklung

realistisch, von einem demographisch bedingten Anstieg der Kosten auszugehen.

${ }^{35}$ Siehe wiederum das Zitat aus Fußnote 29: Auch die Fallzahlen (in Relation zur beitragspflichtigen Bevölkerung) werden hier unter der Hand als konstant unterstellt.

${ }^{36}$ In den Niederlanden geschieht eine solche Mengenkontrolle implizit insofern, wie die Zulassung von zusätzlichen Heimpflegeplätzen anhand fester Quoten in bezug auf die Anzahl der alten Menschen erfolgt. 
der Beitragslast anheimzugeben. Eine solche Variante soll hier nicht als konkretes Gegenmodell präsentiert und ausgeführt werden. Ihre Andeutung dient allein dazu, den sozialpolitischen Widersinn der tatsächlich gewählten Lösung zu verdeutlichen, die den Beitragssatz - zumindest scheinbar - zum absoluten Fixpunkt des Systems erhebt. ${ }^{37}$

In Hinblick auf die übrigen Effizienzaspekte gelten die in bezug auf die Niederlande angestellten Erörterungen weitgehend analog, so daß hier eine komprimierte Behandlung ausreicht. Bezüglich der Beitragsgestaltung sind folgende Abweichungen anzumerken:

- $\quad$ Anders als in den Niederlanden bemessen sich die proportionalen Beiträge nicht nach dem gesamten, sondern allein nach dem Lohneinkommen. ${ }^{38}$ Der Zusammenhang zwischen Beitragshöhe und effektiver Leistungserwartung wird dadurch (noch) weniger eng.

- $\quad$ Ein Freibetrag bei der Beitragsbemessung existiert nicht. Dadurch sind auch solche Personen beitragspflichtig, die aufgrund des ohne Versicherung zu erwartenden Sozialhilfeanspruchs im Pflegefall keinen eindeutigen ${ }^{39}$ Nutzen aus der Versicherung ziehen.

- $\quad$ Ein solcher Nutzen ist insbesondere dann nicht anzunehmen, wenn Versicherte damit rechnen müssen, im Ernstfall trotz Versicherung sozialhilfeabhängig zu werden. Zumindest ex post wird die Beitragsbelastung gerade in solchen Fällen als ungerecht und als Schlechterstellung gegenüber dem versicherungslosen Zustand empfunden werden.

- Die nach dem Pareto-Kriterium wünschenswerte obere Beitragsbemessungsgrenze richtet sich - wie oben entwickelt - nach den höchstens anzunehmenden Pflegekosten, geteilt durch die Rentenquote, also das Verhältnis der Rente zum Erwerbseinkommen. Letzteres liegt in der GRV derzeit bei allenfalls $50 \%$;

\footnotetext{
${ }^{37}$ Vgl. auch K.-D. Henke (1991), S. 127f.: "Insoweit führt die politische Forderung nach Beitragssatzstabilität zu einer Festschreibung (...) auf einem historisch zufälligen Niveau."

${ }^{38}$ Im einzelnen folgt die Abgrenzung der beitragspflichtigen Einnahmen der in der Gesetzlichen Krankenversicherung üblichen, siehe auch $\S 57$.

${ }^{39}$ Ein Versicherungsnutzen entsteht auch für Einkommensschwache in Hinblick auf solche Fälle, in denen die Leistungen umfassender sind, als es die der Sozialhilfe wären. Diese Bedingung wird im Bereich der ambulanten Pflege evtl. mitunter erfüllt sein. Insoweit wäre das Fehlen des Freibetrags zu rechtfertigen.
}

${ }^{40}$ vgl. J. Frerich (1990), S. 162 
in Hinblick auf mögliche ergänzende Alterseinkünfte seien hier $60 \%$ unterstellt. Wird der Höchstschaden typisierend bei DM 4.500 als monatlichem Satz für die stationäre Pflege angesetz $t^{41}$, so sollte die Beitragsbemessungsgrenze bei DM 7.500 liegen. ${ }^{42}$ Mit tatsächlich DM 5.700 in Analogie zur Gesetzlichen Krankenversicherung ist die Grenze also auch in Deutschland zu niedrig festgesetzt worden.

- Bezieher von Einkommen oberhalb der Beitragsbemessungsgrenze können ihrer Versicherungspflicht auf privatem Weg Genüge tun. Nach den $\S \S 110$ und 111 wird dafür ein Einheitsbeitrag erhoben, der den Höchstbeitrag der gesetzlich Versicherten nicht übersteigen darf. ${ }^{43}$ Wenn er ihn praktisch unterschreitet, so stellt dies eine zusätzliche Verletzung der nach dem Pareto-Kriterium erwünschten Beitragsstruktur dar. ${ }^{44}$

Die Diskussion um die vermeintliche Gefahr des Moral hazard braucht hier nicht erneut geführt zu werden. Erinnert sei nur an die Feststellung, daß die in Deutschland möglichen Geldleistungen grundsätzlich anfälliger dafür sind, auch ohne wirklichen Bedarf abgerufen oder zulasten des Bedürftigen auf Betreiben von Angehörigen zweckentfremdet zu werden. Wie schon erwähnt, hat der Gesetzgeber zur Abwehr dieser Gefahren Zugangs- und Verlaufskontrollen vorgeschrieben. Ob sie praktisch ausreichend sind, muß sich erst zeigen.

Ebenfalls schon angeklungen ist die Überlegung, daß die in Sachform gewährten Leistungen aufgrund ihrer groben und starren Stufung auf Basis einer rein medizinischen Indikation gelegentlich auch das Maß des Notwendigen überschreiten und insoweit Mitnahmeeffekte auslösen können. Beim Versuch einer Abgrenzung solcher Fälle ist aber zu beachten, daß neben der adäquaten Versorgung des Bedürftigen selbst

\footnotetext{
${ }^{41}$ Freilich sind noch teurere Fälle vorstellbar. Würde man sie hier zur Grundlage nehmen, müßte aber auch ihre Seltenheit ins Kalkül gezogen werden. Damit wäre dann wieder die in Wahrheit gestaffelte Struktur des Pflegerisikos im Spiel, die - wie gesehen - die Modellvorstellung praktisch unanwendbar zu machen geeignet ist...

${ }^{42}$ Zufällig entspricht dies der für die Gesetzliche Rentenversicherung gültigen Bemessungsgrenze, deren Übertragung auf die Pflegeversicherung im Laufe des Gesetzgebungsverfahrens wiederholt vorgeschlagen worden ist.

${ }^{43}$ Eine Mehrbelastung von maximal 50\% dieses Betrags kann auftreten, wenn ein einkommensloser Ehepartner mitversichert werden soll.

${ }^{44}$ Die Folgen des "Ausstiegs" höherer Einkommensgruppen aus der Sozialversicherung beklagt auch A. Spermann (1993), S. 530.
} 
auch eine Entlastung pflegender Angehöriger vom Gesetzgeber als notwendig betrachtet wird..$^{45}$ Daher sowie angesichts der im Durchschnitt geringen Leistungen ist von einer ineffizienten Überversorgung nur in sehr begrenztem Umfang auszugehen.

Eher ist die Frage zu stellen, ob das Leistungsniveau im ambulanten Bereich für einen der niederländischen Situation vergleichbaren Ausbau solcher Dienste ausreicht. Ein solcher wäre erforderlich, um die Reichweite der häuslichen Pflege gegenüber der stationären Unterbringung zu vergrößern und die Heimeinweisung immer dann vermeiden zu können, wenn sie nicht zwingend erforderlich und damit auch ineffizient ist.

Unter dem Aspekt des Wettbewerbs schließlich, der hier für Deutschland wie für die Niederlande nur ergänzend im Kontext der beiderseits betriebenen Reformen am Gesundheitswesen betrachtet wird, stellt die deutsche Pflegeversicherung einen Rückfall dar. ${ }^{46}$ Der einheitliche Beitragssatz bedingt einen ausgabenorientierten Finanzausgleich zwischen den Kassen, der auch die zurechenbaren Verwaltungsaufwendungen einschließt (§ 66). Nach § 111 ist eine ähnliche Konstruktion auch für die an der Pflegeversicherung beteiligten privaten Versicherungsunternehmen vorgesehen.$^{47}$ Ein solches Verfahren aber ist "mit Wirtschaftlichkeitsanreizen auf Kassenebene nicht vereinbar". ${ }^{48}$ Dabei ist freilich zu bedenken, daß eine Änderung in diesem Punkt nicht ausreichen würde, um Konkurrenz zu erzeugen. Um dies zu verdeutlichen, sei im folgenden von der Existenz des Ausgleichs abstrahiert.

In dieser fiktiven Situation ist in Hinblick auf die Möglichkeit sinnvollen Wettbewerbs zunächst von Bedeutung, daß die Leistungen weitgehend von der jeweils gegebenen Pflegestufe, nicht aber von den Pflegekosten abhängen. Wenn es im stationären Bereich gelänge, den Kostenzuschuß unter der Leistungsobergrenze zu halten, dann wohl nur durch eine sehr großzügige Abgrenzung der Kosten für Unterkunft und Verpflegung, die der Heimbewohner selbst zu tragen hat. Ein solcher Effekt wäre aber in Hinblick auf das Schutzziel unerwünscht. Ansonsten und insbesondere für die ambulanten

\footnotetext{
45 siehe etwa Deutscher Bundestag (1993), S. 65 und 67

${ }^{46}$ K. Jacobs (1994), S. 9, spricht von einem "Relikt aus längst überwunden geglaubten Zeiten".

${ }^{47}$ Der Paragraph formuliert Anforderungen an ein zu schaffendes umfassendes "Ausgleichssystem", ohne Details der Ausgestaltung festzulegen. Da aber auch hier einheitliche Beiträge gelten sollen, scheint es sich ebenfalls um einen ausgabeorientierten ex-post Ausgleich zu handeln.
}

${ }^{48}$ V. Graf/J. Hofmann (1991), S. 11 
Leistungen ist ohne weiteres davon auszugehen, daß die niedrigen Leistungsgrenzen stets voll ausgeschöpft werden. Durch die Vereinbarung günstiger Preise mit den Leistungsanbietern sind die Kassen also nicht in der Lage, ihre Ausgaben zu beeinflussen.

Denkbar wäre somit allenfalls ein Wettbewerb in bezug auf den Umfang (oder auch die Qualität) der im Rahmen der Kostengrenze jeweils gebotenen Dienstleistungen. Für den Versicherungsnehmer wäre es attraktiv, einen Anbieter zu wählen, bei dem auf gleicher Pflegestufe mehr Hilfseinsätze abgerufen werden könnten als bei der Konkurrenz. Dies würde sich zwar nicht unmittelbar auf das Kostenvolumen der Versicherung ${ }^{49}$, wohl aber auf die bei den Versicherten anfallenden Kosten des oftmals notwendigen Leistungszukaufs auswirken.

Entscheidender noch ist für die Realisierung der Leistungskonkurrenz die Gestaltung der Verhandlungs- und Vertragbeziehungen zwischen Kassen und Anbietern:

In bezug auf die Heimpflege steht die Wahlfreiheit der Versicherten der Schließung individueller Verträge zwischen Pflegekassen und Pflegeheimen im Wege. Da die Vereinbarung über die Pflegesätze jeweils zwischen dem Heimträger und einem Konsortium von Kostenträgern zu treffen ist, ist es praktisch ausgeschlossen, daß einzelne Kassen sich auf diesem Gebiet einen Wettbewerbsvorteil erarbeiten. Grundsätzlich möglich ist dies hingegen bei den ambulanten Diensten, die die Pflegekassen selbst einkaufen oder auch selbst erstellen und den Versicherten zuweisen können (§ 36).

Die Wirksamkeit der Maßnahmen zur Qualitätssicherung ${ }^{50}$ vorausgesetzt, läge hier also ein unter Effizienzgesichtspunkten willkommener Spielraum zur innovativen und kostengünstigen Gestaltung des Leistungsgeschehens. Da aber mit dessen aktiver Nutzung angesichts des Finanzausgleichs und der wertmäßigen Fixierung der Leistungsansprüche nicht ohne weiteres zu rechnen ist, wird das Bundesministerium für Arbeit und Sozialordnung in $\S 90$ zum Erlaß einer bundeseinheitlichen Gebührenordnung

\footnotetext{
${ }^{49}$ Indirekt ist eine Kostendämpfung denkbar, da der Druck auf erhöhungen des Beitragssatzes c.p vermindert würde.

so siehe Paragraph 80, dessen Vorschriften zur Erfüllung dieser wichtigen und schwierigen Aufgabe eher unzulänglich erscheinen. Das für die Aufstellung von Qualitätsgrundsätzen besteht in erster Linie aus den Geldgebern, zusätzlich sind die Einrichtungsträger beteiligt. Vertreter der Leistungsempfänger, die als einzige unmittelbar an der Qualität interessiert und von ihr betroffen sind, werden lediglich konsultiert.
} 
ermächtigt. Ob durch diese klassische Regulierungsmaßnahme anstelle von Wettbewerbsmechanismen "die Anreize(!) für eine kostengünstige Leistungserbringung weiter gestärkt werden"51, wie es die Bundesregierung verheißt, erscheint äußerst zweifelhaft.

Eine Begrenzung der eigenen Ausgaben ist den Kassen allenfalls dadurch möglich, daß sie auf die Entscheidung zwischen ambulanter und stationärer Pflege Einfluß zu nehmen versuchen. Im wesentlichen beschränkt sich diese Überlegung auf die Pflegestufe II; während auf Stufe I die für eine Bewilligung erforderliche Notwendigkeit der Heimunterbringung in der Regel nicht gegeben ist, besteht auf der dritten Stufe normalerweise kein Unterschied in der Höhe der Versicherungsleistung zwischen beiden Pflegeformen. ${ }^{52}$ Auf Stufe II aber lassen sich durch die Vermeidung einer Heimeinweisung immerhin Versicherungsleistungen von mindestens DM 700 im Monat einsparen. ${ }^{53}$

Auch die zuletzt durchgespielten Effekte stehen indes im Konjunktiv; in Wirklichkeit findet ein Wettbewerb aufgrund des uneingeschränkten Ausgabenausgleichs praktisch nicht statt. Die Grundsatzdiskussion darüber, unter welchen Bedingungen es im Bereich von Pflege und Pflegeversicherung ökonomisch sinnvoll und sozialpolitisch vertretbar ist, Wettbewerb zu initiieren, war nur am Rande Gegenstand dieser Arbeit und wird auch hier nicht geführt. Es ist vielmehr an einigen Punkten illustriert worden, welche systemspezifischen Probleme bei dem Versuch auftreten müßten, die deutsche Pflegeversicherung durch Aufhebung des Finanzausgleichs für den Wettbewerb zu öffnen: Die möglichen effizienzsteigernden Effekte würden durch fortbestehende andere Hemmnisse nur eingeschränkt zur Geltung kommen.

\footnotetext{
SI Deutscher Bundestag (1993), S. 148

${ }^{52}$ Abgesehen von den Härtefallklauseln ist einschränkend nochmals darauf hinzuweisen, daß für Heimpflege im Durchschnitt nur DM 300 weniger gewährt werden dürfen als der für sie wie für die ambulante Lösung gültige Höchstsatz. Insofern besteht für die Kassen auf Stufe III eventuell ein nicht unproblematisches Motiv zur Begünstigung der Heimpflege, wenn sie trotz des Finanzausgleichs ihre Kosten zu senken bestrebt sind.

${ }^{53}$ Herrschte in den Niederlanden Kassenwettbewerb - und dies könnte wie dargestellt zukünftig zunehmend der Fall sein - wäre der Anreiz zugunsten der häuslichen Pflege weitaus stärker, da für den Versicherer ein Großteil der gesamten Differenz zwischen Heimpflegesatz und häuslichen Betreuungskosten auf den Spiel stünde.
} 


\section{Zusammenfassung: Fünfzehn Thesen zum Schluß}

Die folgenden Thesen geben einen stark komprimierten Überblick über wesentliche Schritte und Ergebnisse der Untersuchung:

1. Am Anfang steht die Feststellung, daß eine ökonomische Analyse zum Thema Pflegeversicherung sich der sozialpolitischen Natur des Gegenstandes bewußt sein muß. Abstrakte ökonomische Zielvorstellungen und Verhaltensannahmen werden diesem nicht ohne weiteres gerecht und bedürfen einer sorgfältigen spezifischen Prüfung oder Erweiterung.

2. So führt schon die Analyse des Problems, also die Frage nach den Gründen für einen ungedeckten Bedarf an Sicherung gegenüber dem Risiko Pflegefall, zu einem Bündel verschiedenartiger Ursachen. Neben der Störung der Marktnachfrage durch die Existenz einer staatlichen Mindestsicherung spielen dabei auf seiten der (potentiellen) Versicherungsnehmer auch psychologische, soziale und historische Gründe eine Rolle. Eine gewisse Unsicherheit und Zurückhaltung im Umgang mit dem als neuartig wahrgenommen Risiko ist zudem auch seitens der Versicherer feststellbar. Insgesamt erscheint ein staatlicher Eingriff zur Schließung der Sicherungslücke angezeigt.

3. Im nächsten Schritt bedarf es einer Spezifikation des Sicherungszieles. Aus der Allgemeinheit des Risikos und der Hindernisse bei seiner Absicherung folgt dabei zunächst die Forderung nach einer personell umfassenden Lösung. Weitere Zielkriterien sind die Abgrenzung des Pflegefalls sowie der relative Umfang und die Beschaffenheit der durch ihn ausgelösten Leistungen. Entscheidend ist, daß eine adäquate Versorgung der Betroffenen gewährleistet ist und sich ihr finanzieller Schaden in Grenzen hält.

4. Bei der Aufstellung von Kriterien zur Bewertung einer Sicherungslösung ist der Tatsache Rechnung zu tragen, daß ein egalitärer, also allein vom Bedarf statt von der individuellen wirtschaftlichen Lage her bestimmter Zugang zu Pflegeleistungen gesellschaftlich gewollt sein kann. Eine solche mögliche Zielvorstellung resultiert aus der Einschätzung, daß die adäquate Versorgung im Pflegefall ein Grundbedürfnis befriedigt.

5. Unter Effizienzgesichtspunkten ist zunächst trivialerweise zu verlangen, daß die Leistungszusage für den Pflegefall nicht nur den genannten inhaltlichen Kriterien 
genügt, sondern darin auch als verläßlich gelten und empfunden werden kann. Bei einer Finanzierung aus Steuermitteln ist diese Bedingung nicht erfüllt.

6. Wenn die Verteilung der Finanzierungslasten einer Pflegeversicherung dem ParetoKriterium wenigstens näherungsweise genügen soll, muß bei ihrer Gestaltung die Existenz der staatlichen Mindestsicherung als wesentliches Merkmal der Referenzlage berücksichtigt werden. Da auf die Mindestsicherung aber nur im Falle absoluter Bedürftigkeit zugegriffen werden kann, führt diese Überlegung zur Forderung nach einkommensabhängigen Beiträgen.

7. Die in der Pflegedebatte gegen das Umlageverfahren vorgetragenen Bedenken halten einer Überprüfung nicht stand. Sie verkennen, daß auch die jeweils jüngere Generation einen sofortigen Nutzen daraus zieht, daß die Generation ihrer Eltern versichert ist. Daher erscheint insbesondere die Befürchtung unbegründet, daß einer umlagefinanzierten Pflegeversicherung die Aufkündigung aufgrund steigender Beitragslasten drohe.

8. "Moral hazard" als Effizienzproblem liegt dann vor, wenn sich Versicherte ihren Informationsvorsprung gegenüber der Versicherung zunutze machen, indem sie mehr Leistungen abfordern, als dies bei vollständiger Beobachtbarkeit ihres Verhaltens und entsprechender Beitragsgestaltung der Fall wäre. Die Natur des Pflegefalls und der darauf bezogenen Leistungen (in Sachform) lassen vermuten, daß die Möglichkeiten und Anreize zum Moral hazard auf seiten Pfegebedürftiger sehr beschränkt sind.

9. Andererseits besteht die Möglichkeit, daß ein Verhalten im Sinne des Moral hazard von den Angehörigen Pflegebedürftiger ausgeht, die vom Pflegefall in gewisser Weise mitbetroffen sind. Im Mittelpunkt der Debatte steht dabei die Vorstellung, daß Angehörige durch die Existenz einer Pflegeversicherung dazu ermuntert würden, eine Heimeinweisung zu betreiben. Eine differenzierte Analyse führt zu dem Ergebnis, daß ein solcher Anreiz durch die Pflegeversicherung nur sehr bedingt ausgelöst wird. Als gegenläufiger Effekt muß zudem bachtet werden, daß die Versicherung den Verzicht auf die Heimeinweisung vielfach erleichtern kann.

10. Die mit der niederländischen Pflegeversicherung tatsächlich verfolgten Ziele entsprechen - soweit sie rekonstruierbar sind - weitgehend dem allgemein aufgestellten Katalog. Auch das Postulat eines gleichen Zugangs zu Pflegeleistungen hat dabei eine gewisse Rolle gespielt. 
11. Die niederländische Pflegesozialversicherung hat in den Jahrzehnten ihres Bestehens eine Reihe recht wesentlicher Veränderungen erfahren; ihre Grundstruktur ist davon aber unberührt geblieben. Ein Systemwechsel zeichnet sich auch gegenwärtig nicht ab, obwohl das Gesundsheitswesen insgesamt Schauplatz von Reformprozessen mit ungewissem Ausgang ist.

12. Im Ergebnis wird die niederländische Pflegeversicherung dem Sicherungsziel weitgehend gerecht. Dies gilt insbesondere für die Breite und Vielfalt des Leistungsspektrums und auch unter Qualitätsgesichtspunkten. Abstriche von diesem Befund sind insofern zu machen, als aufgrund einer strengen Kapazitätskontrolle nicht alle Leistungsansprüche eingelöst werden konnten und können. Neuerdings ist aber das Bemühen erkennbar, die Engpässe zu beheben. Eine weitere Zielverfehlung kann darin erblickt werden, daß das Einkommen Pflegebedürftiger nur bedingt geschützt ist.

13. Zwar wird die niederländische Pflegeversicherung aus einkommensabhängigen Beiträgen finanziert, dem Pareto-Kriterium genügt die Finanzierungsweise - vor allem aufgrund einer sehr niedrigen Beitragsbemessungsgrenze - gleichwohl nicht. Bei einer entsprechend motivierten Umgestaltung des Beitragssystems müßte auch berücksichtigt werden, daß auch die Selbstbeteiligung im Pflegefall mit dem Einkommen variiert.

14. Ein versicherungsinduzierter "Heimsog" im Sinne der These vom Moral hazard ist in den Niederlanden nicht feststellbar. Der starke Zuwachs an Heimplätzen in den 70er Jahren ist vor allem dem sehr niedrigen Ausgangsniveau geschuldet, und inzwischen ist die Nachfrage nach Heimpflege in den Niederlanden sogar deutlich geringer als in der Bundesrepublik ohne kollektive Pflegeversicherung.

15. Eine erste Bewertung der für die Bundesrepublik beschlossenen gesetzlichen Pflegeversicherung läßt im Hinblick auf das Sicherungsziel gravierende Mängel erkennen. Unter der anhaltenden Debatte darüber, $o b$ eine solch umfassende Lösung überhaupt wünschenswert sei, hat das Wie ihrer Ausgestaltung insofern gelitten, als das Ergebnis halbherzig erscheint. Sind schon die Leistungen im Einführungszeitpunkt zu knapp, um die erwünschte Schutzwirkung zu entfalten, so sind die Bedingungen ihrer zukünftigen Anpassung an veränderten Bedarf und damit die Frage der Verläßlichkeit des Arrangements erst recht prekär. Insofern stellt sich in bezug auf die deutsche Pflegeversicherung in der Tat die Frage, ob sie - ohne deutliche Korrekturen - auf Dauer Bestand haben wird. 


\section{Abkürzungen}

AWBZ - $\quad$ Algeme Wet Bijzondere Ziektekosten (Allgemeines Gesetz über besondere Krankheitskosten)

BMA - $\quad$ Bundesministerium für Arbeit und Sozialordnung

BMF $\quad-\quad$ Bundesministerium der Finanzen

BMFuS - $\quad$ Bundesministerium für Familie und Senioren

BMJFFG - $\quad$ Bundesministerium für Jugend, Familie, Frauen und Gesundheit

BSHG - $\quad$ Bundessozialhilfegesetz

CBS - Centraal Bureau voor de Statistiek

CRG - Centrale Raad voor Gezinsverzorging (ehem. Dachverband hauswirtschaftliche Hilfe)

DIW - $\quad$ Deutsches Institut für Wirtschaftsforschung

GKV - $\quad$ Gesetzliche Krankenversicherung

GRV - $\quad$ Gesetzliche Rentenversicherung

hfl $\quad-\quad$ niederländische Gulden

IZW - Informatiecentrum Zorg en Welzijn

LVT - $\quad$ Landelijke Vereniging voor Thuiszorg (Dachverband ambulante Dienste)

NKV - Nationale Kruisvereniging (ehem. Dachverband häusliche Pflege) 
NZI - Nationaal Ziekenhuisinstituut (Forschungseinrichtung des Krankenhauswesens)

PflegeVG - Pflegeversicherungsgesetz

SER - $\quad$ Sociaal-Economische Raad (Beratungsgremium)

SIG - $\quad$ Stichting Informatiecentrum Gezondheidszorg

SVR - Sachverständigenrat zur Begutachtung der gesamtwirtschaftlichen Entwicklung

VNG - Vereniging van Nederlandse Gemeenten (Kommunalverband)

WZV - Wet Ziekenhuisvoorzieningen (Gesetz über die Errichtung von stationären Einrichtungen)

ZFR - Ziekenfondsraad (Beratungsgremium) 


\section{Literatur}

AARON, H. (1981), Economic Aspects of the Role of Government in Health Care, in: J. an der Gaag/M. Perlman (Hg.), Health, Economics and Health Economics, Amsterdam, New York, Oxford

ABHOLZ, H.-H. (1993), Wege aus der Fortschrittsfalle, in: Soziale Sicherheit, 42. Jg., Heft 5, S. 147

ALBER, J. (1990), Pflegebedürftigkeit im Spiegel der öffentlichen Meinung, in: Sozialer Fortschritt, 38. Jg., Heft 9, S. 211

ALBERS, W. (1993), Können die Widerstände gegen die Einführung einer Pflegeversicherung überwunden werden? in: Sozialer Fortschritt, 42. Jg., Heft 1, S. 1

ALLEMEYER, J. (1993), Die Pflegeversicherung der CDU/FDP Koalition - eine gigantische Mogelpackung (Hektographierte Veröffentlichung der Behörde für Arbeit, Gesundheit und Soziales), Hamburg

ALLEMEYER, J. (1994), Die Chance zu Reformen, in: Das Altenheim, 33. Jg., Heft 7, S. 490

ANDRIESSEN, L. (1984), Erfahrungen mit der Versicherung der Pflegekosten in den Niederlanden, in: Zeitschrift für Sozialreform, 30. Jg., S. 366

ARBEITSGEMEINSCHAFT DER SPITZENVERBÄNDE DER FREIEN WOHLFAHRTSPFLEGE DES LANDES NRW (1986), Verbesserung der Stellenschlüssel in den Heimen der Altenhilfe im Bereich Gerontopsychiatrie zur Vermeidung des Pflegenotstandes, Düsseldorf

BAART, S. (1993), Budget voor thuiszorg moet juist worden uitgebreid, in: De Volkskrant vom 8. Juli

BAKKER, B.B./de KAM, C.A. (1992), Het plan-Simons: rijp voor de schroothoop? in: Openbare uitgaven, no. 5, S. 213 
BAKKER, F.M./van de VEN, W.P.M.M./van VLIET, R.C.J.A. (1993), Simons' vrijwillige eigen risico, in: Economisch-Statistische Berichten vom 1. Dezember, S. 1105

BAYERISCHERÜCK(1991), Sozial-und Privatversicherung: Pflege-Zukunftsthema Nr. 1 (Expertengespräch 11 vom 22. März), München

BECKER, G.S. (1981), Altruism in the Familiy and Selfishness in the Market Place, in: Economica 48, S. 1

van der BENT, J. (1989), Onderzoek regionale verschillen verpleeghuizen, (Nationaal Ziekenhuisinstituut) Utrecht

BERTHOLD, N. (1990), Sozialpolitik zwischen ökonomischer und politischer Rationalität, in: Hamburger Jahrbuch für Wirtschafts- und Gesellschaftspolitik, 35. Jg., Tübingen, S. 171

BLOMMESTIJN, P.J. (1990), Ouderenbeleid in Nederland van 1955 tot 1985, Enschede

BÖHM-BAWERK, E. von (1924), Nachteilige Wirkungen des freien Wettbewerbs, in: F.X. Weiss (Hg.), Gesammelte Schriften von Eugen von Böhm-Bawerk, Wien, S. 475

de BOER, N. (1992), Simons: het gaat om een meer dynamische gezondheidszorg, in: Staatscourant nr. 61 vom 26. März 1992

BÖRSCH-SUPAN, A. (1992), Der Wohnungskonsum älterer Mitbürger: Wie lange selbständig? Wie oft in Mehrpersonenhaushalten? in: R. Hujer/H. Schneider/W. Zapf (Hg.), Herausforderungen an den Wohlfahrtsstaat im strukturellen Wandel, Frankfurt/M., New York, S. 143

BOHN, K. (1989), Das Pflegerisiko - privat- oder sozialversicherungsrechtliche Vorsorge vor den finanziellen Folgen? in: Versicherungswirtschaft, 44.Jg., Heft 3, S. 172 
BOOM, R.C./SUURMEIJER, T.P.B.M. (1989), Thuiszorg in Beweging, Groningen

BRANDT, H. (1987), Therapie und Pflege kranker und hilfloser Heimbewohner, in: H. Brandt/E.-M. Dennebaum/W. Rückert (Hg.), Stationäre Altenhilfe, Freiburg, S. 119

BRANDT, H. (1987a), Der Wunsch des alten Menschen nach stationärer Betreuung, in: ders./E.-M. Dennebaum/W. Rückert (Hg.), Stationäre Altenhilfe, Freiburg, S. 30

BRAUN, H. (1987), Pflegebedürftigkeit als sozialer Tatbestand: Zwischen privater Bewältigung und gesellschaftlicher Problemverarbeitung, in: H.Brandt/E.-M. Dennebaum/W. Rückert (Hg.), Stationäre Altenhilfe, Freiburg, S. 11

BREYER, F. (1991), Verteilungswirkungen unterschiedlicher Formen der Pflegevorsorge, in: Finanzarchiv Neue Folge Bd. 49 1991/92, Heft 1, S. 84

BREYER, F. (1994), Verteilungswirkungen unterschiedlicher Formen der Pflegevorsorge: Replik zu Eisen, in: Finanzarchiv Neue Folge Bd. 51, Heft 1, S. 73

BREYER, F./ZWEIFEL, P. (1992), Gesundheitsökonomie, Berlin, Heidelberg van den BROEK, P.A.M. (1993), Modernisering zorgsector en de zorgverzekering (2 Teile), in: Periodiek voor sociale verzekering (PS) nr. 12 vom 16. Juni und nr. 13 vom 30. Juni, S. 872 bzw. 949

BRUINSMA, J. (1993), Contributie kruishulp volgend jaar omhoog, in: de Volkskrant vom 5. November

BRUINSMA, J. (1993a), Simons wil eigen risico voor AWBZ, in: de Volkskrant vom 5. November

BUCHHOLZ, W./WIEGARD, W. (1992), Allokative Überlegungen zur Reform der Pflegevorsorge, in: Jahrbücher für Nationalökonomie und Statistik, Band 209/5-6, S. 441 
BUNDESMINISTER FÜR JUGEND, FAMILIE, FRAUEN UND GESUNDHEIT (1986), Vierter Familienbericht, Bonn

BUNDESMINISTERIUM FÜR ARBEIT UND SOZIALORDNUNG (BMA) (1990), Die gesetzliche Krankenversicherung in der Bundesrepublik Deutschland im Jahre 1989, Bonn

BUNDESMINISTERIUM FÜR ARBEIT UND SOZIALORDNUNG (BMA) (1992), Informationen zum Stand der Pflegeversicherung, Bonn

BUNDESMINISTERIUM FÜR ARBEIT UND SOZIALORDNUNG (BMA) (1993), Pflegeversicherung kommt, Bonn

CENTRAAL BUREAU VOOR DE STATISTIEK (CBS) (1991), Statistisch jaarboek 1991, 's Gravenhage

CENTRAAL BUREAU VOOR DE STATISTIEK (CBS) (1993), Statistical Yearbook of the Netherlands 1993, The Hague

CENTRALE RAAD VOOR DE VOLKSGEZONDHEID (1982), Interim-advies inzake het functioneren van verpleeghuizen, Rijswijk

COMMISSIE VOOR DE BELASTINGSHERZIENING (1991), Graag of niet samenvatting, 0.0 .

CULYER, A.J. (1991), The Normative Economics of Health Care Finance and Provision, in: A. McGuire/P. Fenn/K. Mayhew (Hg.), Providing Health Care: The Economics of Alternative Systems of Finance and Delivery, Oxford, S. 65

DANIELS, N. (1981), Health Care Needs and Distributive Justice, in: Philosophy and Public Affairs, Vol. 10, No. 2, S. 146

DAS PARLAMENT (1993), Erste Lesung des Gesetzentwurfes zur Pflegefallversicherung am 1. Juli 1993 im Deutschen Bundestag, Ausgaben 29 und 30 vom 16. und 23. Juli 
DEKKER, P./ESTER, P. (1993), Social and Political Attitudes in Dutch Society, (Social and Cultural Planning Office) Rijswijk

DENNEBAUM, E.-M./RÜCKERT, W. (1987), Ambulante und stationäre Pflege - Konkurrenz oder Ergänzung? in: H.Brandt und dieselben (Hg.), Stationäre Altenhilfe, Freiburg, S. 163

DEUTSCHER BUNDESTAG (1984), Bericht der Bundesregierung zu Fragen der Pflegebedürftigkeit, Drucksache 10/1943 vom 5. September

DEUTSCHER BUNDESTAG (1993), Gesetzentwurf der Fraktionen der CDU/CSU und F.D.P. - Entwurf eines Gesetzes zur sozialen Absicherung des Risikos der Pflegebedürftigkeit, Drucksache 12/5262

DEUTSCHES INSTITUT FÜR WIRTSCHAFTSFORSCHUNG (DIW) (1993), ReRegulierung des Marktes für Altenpflege erforderlich, in: DIW-Wochenbericht, 60. Jg., Nr. 31 vom 5. August

DIECK, M. (1987), Gewalt gegen ältere Menschen im familiären Kontext - Ein Thema der Forschung, der Praxis und der öffentlichen Information, in: Zeitschrift für Gerontologie, 20. Jg., S. 305

van DUUREN, R./POSTHUMA, B.H./RUYGT, F.A.M. (1989), Marktwerking, normuitkeringen en risico selectie, in: Economisch-Statistische Berichten vom 13. Dezember 1989 , S. 1232

EERSTE KAMER (1991), Kwaliteit van Zorg (14de uitgebreide commissie vergadering vom 9. Dezember 1991) in: Handelingen 2 vergaderjaar 1991-1992

EISEN, R. (1988), "Versicherungsprinzip" und Umverteilung - Einige theoretische Überlegungen zu den Grenzen des Versicherbaren, in: G. Rolf/P.B. Spahn/G. Wagner (Hg.), Sozialvertrag und Sicherung, Frankfurt, New York 1988, S. 117

EISEN, R. (1992), Alternative Sicherungsmöglichkeiten bei Pflegebedürftigkeit, in: Sozialer Fortschritt, 41. Jg., Heft 10, S. 236 
EISEN, R. (1994), Verteilungswirkungen unterschiedlicher Formen der Pflegevorsorge: Kommentar zu Breyer (1991/92), in: Finanzarchiv Neue Folge, Band 51, Heft 1, S. 68

ELBERSE, W.P. (1989), Vraag en aanbod verpleeghuiszorg in de regio's Den Haag en Rijnmond, Utrecht

FELDERER, B. (1992), Die langfristige Entwicklung einer gesetzlichen Pflegeversicherung, (Bayerische Rück) o.O.

FELKNER, C./STEIN, P. (1992), Ausgestaltung einer Organisationsreform der gesetzlichen Krankenversicherung, in: Wirtschaftsdienst, 72. Jg., Heft III, S. 159

FÖRDERKREIS BAD NAUHEIMER GESPRÄCHE (1985), Versicherung und Versorgung für den Pflegefall, Mainz

FRANK, W. (1987), Notwendigkeit, Ausgestaltung und Auswirkungen einer Pflegeversicherung auf den stationären Bereich, in: Brandt, H./Dennebaum, E.-M./Rückert, W. (Hg.), Stationäre Altenhilfe, Freiburg, S. 227

FRANKFURTER INSTITUT (1991), Pflegefall-Vorsorge: Statt altem Teufelskreis endlich Zukunftsweisendes aufbauen, in: Argumente zur Wirtschaftspolitik, Nr. 37, Juli

FRERICH, J. (1990), Sozialpolitik, 2. Auflage, München, Wien

GABANYI, M./MAY, S./SCHNEIDER, M. (1992), Absicherung des Pflegerisikos am Beispiel ausgewählter europäischer Länder, (Bundesministerium für Arbeit und Sozialordnung) Bonn

GEITNER, D. (1985), Stand der Richtliniendiskussion mit dem Bundesaufsichtsamt für das Versicherungswesen zur Pflegefallversicherung aus der Sicht der privaten Lebensversicherung, in: Förderkreis Bad Nauheimer Gespräche (Hg.), Versicherung und Versorgung für den Pflegefall, Mainz, S. 27 
GÖRRES, S. (1993), Fragen der Qualitätssicherung in Geriatrie und stationärer Altenhilfe - ein Problemaufriß, Manuskript eines Vortrags auf der KDA-Tagung "Zur Zukunft der stationären Altenhilfe", September

GÖSSLING, S. (1987), Teilstationäre Dienste - Möglichkeiten und Probleme, in: H. Brandt/E.-M. Dennebaum/W. Rückert (Hg.), Stationäre Altenhilfe, Freiburg, S. 182

GOUDRIAAN, F.G.W. (1990), Substitutie van voorzieningen in de ouderenzorg, Rijswijk

GOUDRIAAN, F.G.W./WENNINK, J. (1992), In het teken van de klant (II), in: Tijdschrift voor gezondheid en politiek, Januar, S. 3

GRAF, V./HOFMANN, J. (1991), Herausforderung für die GKV?, in: Arbeit und Sozialpolitik, Heft 1-2, S. 6

GROENEN, W./van der WOUDEN, R. (1991), Nursing Homes in the Netherlands: Costs and Policy, in: Finanzarchiv 1991/92, Heft 1, S. 104

GROSSJOHANN, K./ZÖLLNER, D. (1984), Soziale Sicherung bei Pflegebedürftigkeit in europäischen Nachbarländern, Bonn

GRÜNERT, J. (1981), Kostenschätzung statt politischer Diskussion, in: Sozialer Fortschritt, 30. Jg., Heft 5, S. 107

ten HAAFT, G. (1988), Tijdelijke opname werkt substituerend in verpleeghuizen, in: Het Ziekenhuis Nr. 17 vom 28. September, S. 756

HACKMANN, J. (1978), Zulässige und unzulässige Schlußfolgerungen nach dem Pareto-Kriterium, in WiSt, Heft 12, S. 557

HÄNLEIN, A. (1992), Die Heranziehung Unterhaltspflichtiger bei langwährender Pflegebedürftigkeit Volljähriger nach BSHG und BGB, Diss. Bielefeld 
HASE, F. (1992), Benachteiligung der Familie durch die Rentenversicherung? in: Wirtschaftsdienst, 72. Jg., Heft X, S. 526

HENGSBACH, F./MÖHRING-HESSE, M. (1992), "Mehr Markt" macht nicht gesund, in: Sozialer Fortschritt, 41. Jg., Heft 5-6, S. 119

HENKE, K.-D. (1990), die finanzielle Absicherung des Pflegerisikos (Manuskript), Hannover

HENKE, K.-D. (1991), Langfristige Finanzierbarkeit der Gesetzlichen Krankenversicherung, in: F.W. Schwartz et al (Hg.), Public health, Berlin, Heidelberg, New York, S. 125

HEUBECK, G. (1982), Versicherungswissenschaftliche Untersuchung, in: H. F. Zacher (Hg.), Die Rolle des Beitrags in der sozialen Sicherung, Berlin, S. 289

HOLLANDER, E./de VISSER, J.H. (1991), Ouderenzorg veel meer thuis, in: Medisch Contact, Nr. 4, S. 115

IGL, G. (1986), Bemerkungen zu einer Neuordnung der Sicherung bei Pflegebedürttigkeit, in: Sozialer Fortschritt, 35. Jg., Heft 9, S. 193

INFRATEST SOZIALFORSCHUNG (1992), Hilfe- und Pflegebedarf in Deutschland (Studie im Auftrag des Bundesministeriums für Familie und Senioren), München

INFRATEST SOZIALFORSCHUNG (1993), Möglichkeiten und Grenzen selbständiger Lebensführung - Zusammenfassung wichtiger Ergebnisse der Repräsentativerhebung, München

INFORMATIECENTRUM ZORG EN WELZIJN (1992), Eigen bijdrage-regeling AWBZ (Loseblattsammlung), o.O. (Utrecht)

JACOBS, K. (1993), Sinnvoller Kassenwettbewerb in der GKV, in: Wirtschaftsdienst, 73. Jg., Heft XI, S. 597 
JACOBS, K. (1994), Zur Kohärenz von gesetzlicher Pflegeversicherung und anderen Zweigen der Sozialversicherung, Erste Fassung Manuskript zum Kolloquium "Auswirkungen der Einführung der gesetzlichen Pflegeversicherung, Bremen, Oktober

JOPEN, C. (1990), Personalschlüssel in der stationären Altenhilfe in der Bundesrepublik Deutschland, in: Nachrichtendienst des Deustchen Vereins für öffentliche und private Fürsorge, 70. Jg., Heft 10, S. 352

de KAM, C.A. (1993), Zorg, in: Jaarboek overheids uitgaven 1994, Schoonhoven, S. 65

KARTEN, W. (1993), Das Einzelrisiko und seine Kalkulation, Wiesbaden

KASKE, W. (1985), Grundprobleme privater Pflegefallversicherungen - die aktuelle Situation aus der Sicht der Privaten Krankenversicherung, in: Förderkreis Bad Nauheimer Gespräche (Hg.), Versicherung und Versorgung für den Pflegefall, Mainz, S. 35

KASTELEIN, M./WEVERS, C.J.W./WISSINK, G.J. (1985), Samenwerking, een indicatie voor betere verpleeghuiszorg? Leiden

KESS,W. (1980), Die Pflege-(volks-)versicherung in den Niederlanden, in: Die Ersatzkasse, Heft 9, S. 381

KIKKERT et al. (1966), Verslag van het mondeling overleg, in: Handelingen 2de Kamer, vergaderjaar 1966-67, 8457 (nr. 10)

KLAASSEN-van den BERG JETHS, A. (1991), Zorgvoorzieningen voor ouderen, in: Ministerie van Welzijn, Volksgezondheid en Cultuur (Hg.), Ouderen straks, Rijswijk, S. 12

KLIE, T. (1987), Heime im normativen Konflikt, in: H. Brandt/E.-M. Dennebaum/W. Rückert (Hg.), Stationäre Altenhilfe, Freiburg, S. 47

KLUWER SOCIALE VERZEKERINGSWETTEN (1989), Deel 7: AWBZ (Loseblattsammlung), o.O. (Deventer) 
KLUWER ZORG EN DIENSTVERLENING (1990), verpleeghuizen, in: Deel 2: Ouderenbeleid (Loseblattsammlung), o.O. (Deventer)

KNÖPFEL, G. (1985), Beistand und Rücksicht zwischen Eltern und Kindern (Paragraph 1618a BGB), in: Familienrechts-Zeitschrift (FamRZ), Heft 6, S. 554

KRUG, W./REH, G. (1992), Pflegebedürftige in Heimen, (Bundesministerium für Familie und Senioren) Stuttgart, Berlin, Köln

KURATORIUM DEUTSCHE ALTERSHILFE (1979), Gutachten über die stationäre Behandlung von Krankheiten im Alter und über die Kostenübernahme durch die gesetzlichen Krankenkassen, Köln

LAMPERT, H. (1985), Lehrbuch der Sozialpolitik, 1. Auflage, Berlin Heidelberg LAMPERT, H. (1991), Lehrbuch der Sozialpolitik, 2. Auflage, Berlin, Heidelberg LANDELIJKE VERENIGING VOOR THUISZORG (LVT) (1990a), Registratie indicatie thuiszorg, Bunnik

LANDELIJKE VERENIGING VOOR THUISZORG (LVT) (1990), Community nursing and home help care in the Netherlands, Bunnik

LANDELIJKE VERENIGING VOOR THUISZORG (LVT) (1991), Jaarverslag 1990, Bunnik

LANDELIJKE VERENIGING VOOR THUISZORG (LVT) (1993), Meerjaarenraming - Thuiszorg in beeld, Bunnik

LAURS, P.J.N./TEUBEN, G.B. (1990), Intramurale zorgverlening aan hulpbehoevende ouderen, in: Nederlands Juristenblad Speciaal vom 13. Dezember, S. 1797

MACKSCHEIDT, K. (1985), Über die Belastbarkeit mitSozialversicherungsbeiträgen aus der Sicht der Steuerwiderstandsforschung, in: W. Schmähl (Hg.) Versicherungsprinzip und soziale Sicherung, Tübingen, S. 27 
MEIJER, M.C.F. et al. (1989), Oort - Herziening belastings- en premieheffing en gevolgen voor arbeidvoorwaarden sociale verzekeringen en pensioenen, in: PS 7 (Sonderheft), März, S. 11

MEYER, DIRK (1989), Ordnungs- und sozialpolitische Aspekte einer Absicherung des Pflegefallrisikos, in: Wirtschaftsdienst, 69. Jg., Heft VI, S. 299

van MIERLO, J.G.A. (1991), Een alternatief voor het plan-Simons, in: EconomischStatistische Berichten vom 20. November, S. 1164

MILTENBURG, T./MENSINK, J./RAMAKERS, C. (1993), Experiment Clientgebonden Budget Verzorging \& Verpleging - Beleidsgerichte evaluatie, Nijmegen

MINISTERIE VAN FINANCIEN (1993), Verslag van de Werkgroep Grondslag Financiering Ziektekosten, Den Haag

MÜLLER, JOACHIM (1990), Ausgestaltung und Reform des niederländischen Gesundheitswesens - Parallelen und Unterschiede zur Entwicklung in der Bundesrepublik Deutschland, in: Die Sozialversicherung, 45. Jg., Nr. 8, S. 197

MÜLLER, WOLFGANG (1988), Das Versicherungsprinzip - Zum Gefahrengemeinschaftsmythos in Versicherungstheorie und -praxis, in: G. Rolf/B. Spahn/G. Wagner (Hg.), Sozialvertrag und Sicherung, Frankfurt/M., New York, S. 129

NATIONAAL ZIEKENHUISINSTITUUT (NZI) (1989), Relatie zorgproces en inzet van middelen in verpleeghuizen, Utrecht

NATIONAAL ZIEKENHUISINSTITUUT (NZI) (1990), De intramurale gezondheidszorg in cijfers per 1 januari 1990, Utrecht

NATIONAALZIEKENHUISINSTITUUT(NZI) (1991), Statistiek personeels-sterkte 1990 (3 Teile), Utrecht

NATIONALE KRUISVERENIGING (NKV) (1989), Kruiswerk: voor zorg en voorzorg, Bunnik 
NATIONALE RAAD VOOR DE VOLKSGEZONDHEID (1988), Kanttekeningen bij de nota Verandering verzekerd, Zoetermeer

NELL, M. (1992), Versicherungsinduzierte Verhaltensänderungen von Versicherungsnehmern (Diss. Universität Hamburg), Hamburg

OBERENDER, P. (1986), Ökonomische Aspekte des Pflegefallrisikos, in: W. Gitter/ P. Oberender (Hg.), Pflegefallrisiko, Bayreuth

OECD (1990), Health care expenditure and other data, in: OECD (Hg.), Health Care Systems in Transition, Paris, S. 119

OECD (1992), The Reform of Health Care - A Comparative Analysis of Seven OECD Countries, Paris 1992

OFFE, C./SCHMÄHL, W. (1992), Forschungsvorhaben "Die gesetzliche Absicherung des Pflegefallrisikos - Eine Untersuchung der Konsensbildungsprozesse und Wirkungen einer Pflegeversicherung (Manuskript), Bremen

O.V. (1992), Zorg in Nederland 1992 (Kurzfassung Financieel Overzicht Zorg), Rijswijk

O.V. (1992a), Plannen Simons desastreus voor voorstellen commissie Stevens, in: Welwezen, nr. 2, S. 5

OXLEY, H. (1992), The performance of health systems in OECD countries, in: OECD (Hg.), Progress in Structural Reform. An Overview, Paris, S. 59

PARINGER, L. (1983), Economic Incentives in the Provision of Long-Term Care, in: Jack A. Meyer (Hg.), Market Reforms in Health Care, Washington, London, S. 119

PAULY, M.V. (1990), The Rational of Long-Term Care Insurance, in: Journal of Political Economy, Vol. 98, no. 1, S. 153 
PERIODIEK VOOR SOCIALE VERZEKERING (PS) (1982), Eigen bijdrageregeling AWBZ, in: Ausgabe 20 vom 12. Oktober 1982, S. 1047

PERIODIEK VOOR SOCIALE VERZEKERING (PS) (1990), Tariefstructuur, in: Ausgabe 3 (speciaal), januari, S. 107

PETERSEN, G. (1989), Sozialökonomik, Stuttgart, Berlin, Köln

PIRKL, A. (1986), Absicherung des Lebensrisikos der Pflegebedürftigkeit: Eine sozialund ordnungspolitische Herausforderung, in: W. Gitter/P. Oberender (Hg.), Pflegefallrisiko, Bayreuth, S. 7

POEISZ, J.J./VELUWENKAMP, D. (1991), Wat betekent de stelselherziening voor instellingen? Utrecht

POSKE, D. (1984), Sicherung bei Pflegebedürftigkeit im Rechtsvergleich mit den Niederlanden - Modell oder Fehlentwicklung? in: Nachrichtendienst des Deutschen Vereins für öffentliche und private Fürsorge, 64. Jg., S. 290

PRESSE- UND INFORMATIONSAMT DER BUNDESREGIERUNG (1994), Pflegeversicherungsgesetz, in: Sozialpolitische Umschau Nr. 190 vom 2. Mai, S. 1

PRINZ, A. (1987), Pflegebedürftigkeit als ökonomisches Problem, Spardorf

REIJMERINK, W. (1990), Nietreguliere zorgvoorzieningen voor ouderen, Utrecht

REINHARDT, U.E. (1990), [Comment on B. Jönsson: What can Americans learn from Europeans?], in: OECD (Hg.), Health Care Systems in Transition, Paris, S. 105

RIBHEGGE, H. (1990), Denkfehler zum Thema Alterssicherung: Kapitaldeckungsvs. Umlageverfahren, in: Jahrbuch für Sozialwissenschaft 41, S. 359

RIVLIN, A.M./WIENER, J.M. (1988), Caring for the Disabled Elderly, Washington 
ROBINE, J.-M./CAMBOIS, E. (1994), An International Comparison of Trends in Disability-Free Life Expectancy, Manuskript zum Vortrag beim Internationalen Workshop "Alternatives in Financing Long-Term Care, Frankfurt/Main, 31. August bis 2. September

von RÖTEL, M. et al. (1980), Die Pflegeheim-Versorgung älterer Menschen in den Niederlanden im Vergleich zur Bundesrepublik - Teilergebnisse einer Studienreise, Köln

ROLF, G./SPAHN, P.B./WAGNER, G. (1988), Wirtschaftstheoretische und sozialpolitische Fundierung staatlicher versicherungs- und Umverteilungspolitik, in: dieselben (Hg.), Sozialvertrag und Sicherung, Frankfurt, New York, S. 13

RÜCKERT, W. (1987), Demographische Grundlagen zur Altenhilfe-Planung, in: H. Brandt/E.-M. Dennebaum/W. Rückert (Hg.), Stationäre Altenhilfe, Freiburg, S. 59

RÜCKERT, W. (1987a), Personelle Rahmenbedingungen für eine angemessene Pflege von Heimbewohnern, in: H. Brandt/E.-M. Dennebaum/W. Rückert (Hg.), Stationäre Altenhilfe, Freiburg

RUF, T. (1985), Pflegeversicherung - der falsche Weg, in: Arbeit und Sozialpolitik, S. 223

SACHVERSTÄNDIGENRAT ZUR BEGUTACHTUNG DER GESAMTWIRTSCHAFTLICHEN ENTWICKLUNG (SVR) (1991), Jahresgutachten 1991/92, erschienen als Bundestags-Drucksache 12/1618 vom 18. November

SCANLON, W.J. (1992), Possible Reforms for Financing Long-Term Care, in: Journal of Economic Perspectives, Vol. 6, nr. 3, S. 43

SCHÄFER, D. (1982), Sozialpolitische Untersuchung, in: H.F. Zacher (Hg.), Die Rolle des Beitrags in der sozialen Sicherung, Berlin, S. 331

SCHMÄHL, W. (1992), Zum Vergleich von Umlageverfahren und kapitalfundierten Verfahren zur Finanzierung einer Pflegeversicherung in der Bundesrepublik Deutschland, (Bundesministerium für Familie und Senioren) Stuttgart, Berlin, Köln 
SCHNEEKLOTH, U./POTTHOFF, P. (1993), Hilfe- und Pflegebedürftige in privaten Haushalten, (Infratest-Studie im Auftrag des Bundesministeriums für Familie und Senioren,) Stuttgart, Berlin, Köln

SCHÖNBACH, K.H. (1985), Haben ordnungspolitische Reformkonzepte eine Chance? in: Sozialer Fortschritt, 34. Jg., Heft 2, S. 40

SCHREIBER, L.H./SCHREIBER, C. (1993), Alte Menschen als Ziel krimineller Handlungen in der Familie, in: Zeitschrift für Rechtspolitik, Heft 4, S. 146

SCHÜLKE, H. (1984), Die Finanzierung der Pflegekosten - eine schwierige Aufgabe für die Sozialpolitik, in: Versicherungswirtschaft, 39. Jg., S. 1597

SCHULZ-NIESWANDT, F. (1989), Kritik der "moral hazard"- und "Sog"Erwartungen bei Einführung einer Pflege-Sozialversicherung, in: Sozialer Fortschritt, 38. Jg., Heft 8, Seite 181

SCHULZ-NIESWANDT, F. (1992), Sozialpolitik als "Soziale Politik" - Sozialpolitik als interdisziplinäre Querschnittswissenschaft, in: Sozialer Fortschritt, 41. Jg., Heft 10, S. 243

SCHUT, F.T./van VLIET, R.C.J.A. (1992), Raadsels rond de omslagbijdrage ziektekostenverzekering, in Openbare uitgaven, nr. 2, S. 57

SEILER, D. (1991), Vorschläge zur Pflegeversicherung im Vergleich, in: Wirtschaftsdienst, 71. Jg., Heft VII, S. 350

SINN, H.W. (1977), Die Allokationswirkung der Versicherung, in: Zeitschrift für die gesamte Versicherungswissenschaft, Bd. 66, S. 507

SPAAN, J.M. (1989), Verpleeghuizen in cijfers, (Nationaal Ziekenhuisinstituut) Utrecht

SPERMANN, A. (1993), Allokative und distributive Effekte aktueller Pflegeversicherungsmodelle, in: Wirtschaftsdienst, 73. Jg., Heft X, S. 526 
STAATSBLAD VAN HET KONINKRIJK DER NEDERLANDEN (1990), Algemeen Wet Bijzondere Ziektekosten (zuletzt geändert am 20. Dezember 1989) nr. 176

STICHTING CENTRAAL ADMINISTRATIE KANTOOR AWBZ (1989, 1990, 1991, 1993), Verslag over het jaar 1988, 1989, 1990, 1992, 's-Gravenhage

STICHTING CENTRALE RAAD VOOR GEZINSVERZORGING/NATIONALE KRUISVERENIGING (1990), Diskussienota thuiszorg, Bunnik

STICHTING INFORMATIECENTRUM GEZONDHEIDSZORG (SIG) (1992), Jaarboek verpleeghuizen 1991, Utrecht

STIFTUNG WARENTEST (1991), Sonderheft Versicherungen, Berlin

STIKKER, A.J. (1987), Grensverkenningen - een studie naar de relatie gezinsverzorging/thuiszorg - ziekenhuizen/verpleeghuizen, 's-Gravenhage

STRASSL, W. (1988), Externe Effekte auf Versicherungsmärkten, (Diss. Universität München) Tübingen

THIEDE, R.F. (1990), Die gestaffelte Pflegeversicherung, Frankfurt/M. New York THUROW, L.C. (1974), Cash Versus In-Kind Transfers, in: American Economic Review, Vol. 64, No. 2 (Mai), S. 190

TISMER, K.G. et al. (1975), Psychosoziale Aspekte der Situation älterer Menschen, Stuttgart

TITMUSS, R.M. (1976), Committment to Welfare, 2. Auflage, London

TOETSINGSCOMMISSIE BUDGETTERING VERZEKERAARS (1993), Verslag, Ridderkerk

TWEEDE KAMER (1966), Ontwerp van Wet, in: Handelingen 1965-1966, 8457 nr. 2 
TWEEDE KAMER (1967), Verslag 8ste vergadering vom 24. Oktober 1967, in: Handelingen 1967-1968, Bd. 3, S. 258

TWEEDE KAMER (1982), Verslag vergadering vom 21. Oktober 1982, in: Handelingen 1982-1983, Bd. 4, S. 398

TWEEDE KAMER (1983), Verslag van een mondeling overleg (nebst Beilagen), in: Handelingen 1982-1983, 17600, hoofdstuk XVII, nr. 11-13

TWEEDE KAMER (1990), Nota Ouderen in tel, in: Handelingen 1990-1991, 21814 TWEEDE KAMER (1992), Financieel overzicht zorg 1992, in Handelingen, vergaderjaar 1991-1992, 22311

TWEEDE KAMER (1994), Financieel overzicht zorg 1994, in: Handelingen, vergaderjaar 1993-1994, 23407

ULEER, H.C. (1991), Alternativen zur Pflegeversicherung, in: Orientierungen, Nr. 48 (Juni), S. 53

ULLMANN, C. (1993), Ist die Senkung des Arbeitslosengeldes verfassungswidrig? in: Süddeutsche Zeitung vom 16. September

VAUBEL, R. (1983), Die soziale Sicherung aus ökonomischer Sicht, in: H. Siebert (Hg.), Perspektiven der deutschen Wirtschaftspolitik, Stuttgart u.a., S. 151

VELDKAMP, G.M.J. et al. (1966), Memoire van toelichting, in: Handelingen 2de Kamer 1965-1966, 8457 nr. 3

van de VEN, W.P.M.M. (1990), From Regulated Cartel to Regulated Competition in the Dutch Health Care System, in: European Economic Review, Vol. 34, S. 632

van de VEN, W.P.M.M. (1992), Plan-Dekker is "perestroika" gezondheidszorg, in: Staatscourant Nr. 61 vom 26. März 
van de VEN, W.P.M.M./van VLIET, R.C.J.A. (1992), How can we prevent cream skimming in a competitive health insurance market? in: P. Zweifel/H.E. Frech (Hg.), Health Economics Worldwide, Deventer, S. 23

VERENIGING VAN NEDERLANDSE GEMEENTEN (VNG) (1988), Het besluit indicatie advisering bejaardenoorden en verpleeginrichtingen, 2 . Auflage, 's-Gravenhage

VOLKERT, J. (1991), Sozialpolitik und Wettbewerbsordnung: Die Bedeutung der wirtschafts- und sozialpolitischen Konzeption Walter Euckens für ein geordnetes sozialpolitisches System der Gegenwart, in: Ordo, Bd. 42, Stuttgart, New York

WAGNER, G. (1991), Geringe Unterschiede zwischen privatwirtschaftlicher und sozialversicherungsrechtlicher Vorsorge, in: Arbeit und Sozialpolitik, Heft 3-4, S. 40

WASEM, J. (1991), Der mögliche Beitrag der Versicherungswirtschaft zur Lösung der Pflegefallproblematik - Perspektiven, Probleme, Lösungsansätze, in: Zeitschrift für Versicherungswesen, 42. Jg., Heft 15-16, S. 378

van WEERT, N.J.H.W. (1991), Psychogeriatrische dagbehandeling buiten de muren van het verpleeghuis - een longitudinaal experiment, Nijmegen

WELTEN, J.B.V./ten DAM-van LIESHOUT, C.A. (1987), De verpleeghuisproblemen in een grote stad, in: Tijdschrift voor gerontologie en geriatrie, nr. 18, S. 241

WILLIAMS, A. (1981), Welfare Economics and Health Status Measurement, in: J. van der Gaag/M. Perlman (Hg.), Health, Economics, and Health Economics, Amsterdam, New York, Oxford, S. 271

WINTERS, S. (1991), Verteilungswirkungen einer Gesetzlichen Pflegeversicherung: Eine Replik, in: Wirtschaftsdienst, 71. Jg., Heft IX, S. 478

WINTERS, S. (1992), Pflegeversicherung - das Beispiel der Niederlande, in: Arbeit und Sozialpolitik, Heft 7-8, S. 11 
WINTERS, S. (1993), Blüm und Pareto - Überlegungen zur Finanzierung der Pflegeversicherung, in: Wirtschaftsdienst, 73. Jg., Heft VI, S. 300

WISEMAN, J. (1985), Genesis, Aims and Goals of Social Policy, in: Public Finance and Social Policy - Proceedings of the 39th Congress of the IIPF, Budapest 1983, Detroit, S. 93

WISSENSCHAFTLICHER BEIRAT BEIM BMF (1990), Stellungnahme zur Finanzierung von Pflegekosten, (Bundesministerium der Finanzen) Bonn

ZIEKENFONDSRAAD (ZFR) (1987), Advies nr. 361: Verhoging maximale eigen bijdrage AWBZ, Amstelveen

ZIEKENFONDSRAAD (ZFR) (1989), Advies nr. 440: Harmonisatie eigen bijdrageregelingen verpleeghuizen en bejaardenoorden, Amstelveen

ZIEKENFONDSRAAD (ZFR) (1991), Advies nr. 531: Nota "Kwaliteit van zorg", Amstelveen

ZIEKENFONDSRRAD (ZFR) (1992), Advies nr. 550: Financieel overzicht zorg 1993, Amstelveen

ZIEKENFONDSRAAD (ZFR) (1993), Advies nr. 576: Nota evaluatie stelselwijziging, Amstelveen

ZWEIFEL, P./FELDER, S./LANDOLT, D./NOCERA, S./STRÜWE, W. (1994) Pflegebedürftigkeit im Alter, (Zürcher Kantonalbank) Zürich

ZWEIFEL, P./STRÜWE, W. (1993), Pflegeversicherung und gebundenes Banksparen in einem Zwei-Generationen-Modell, Zürich 
Stephan Winters - 978-3-631-75191-6

Downloaded from PubFactory at 01/11/2019 07:17:47AM

via free access 


\section{FINANZWISSENSCHAFTLICHE SCHRIFTEN}

Band 1 Werner Steden: Finanzpolitik und Einkommensverteilung. Ein Wachstums- und Konjunkturmodell der Bundesrepublik Deutschland. 1979.

Band 2 Rainer Hagemann: Kommunale Finanzplanung im föderativen Staat. 1976.

Band 3 Klaus Scherer: Maßstäbe zur Beurteilung von konjunkturellen Wirkungen des öffentlichen Haushalts. 1977.

Band 4 Brita Steinbach: "Formula Flexibility" - Kritische Analyse und Vergleich mit diskretionärer Konjunkturpolitik. 1977.

Band 5 Hans-Georg Petersen: Personelle Einkommensbesteuerung und Inflation. Eine theoretisch-empirische Analyse der Lohn- und veranlagten Einkommensteuer in der Bundesrepublik Deutschland. 1977.

Band 6 Friedemann Tetsch: Raumwirkungen des Finanzsystems der Bundesrepublik Deutschland. Eine Untersuchung der Auswirkungen der Finanzreform von 1969 auf die Einnahmenposition der untergeordneten Gebietskörperschaften und ihrer regionalpolitischen Zieiadäquanz. 1978.

Band 7 Wilhelm Pfähler: Normative Theorie der fiskalischen Besteuerung. Ein methodologischer und theoretischer Beitrag zur Integration der normativen Besteuerungstheorie in der Wohlfahrtstheorie. 1978.

Band 8 Wolfgang Wiegard: Optimale Schattenpreise und Produktionsprogramme für öffentliche Unternehmen. Second-Best Modelle im finanzwirtschaftlichen Staatsbereich. 1978.

Band 9 Hans P. Fischer: Die Finanzierung des Umweltschutzes im Rahmen einer rationalen Umweltpolitik. 1978.

Band 10 Rainer Paulenz: Der Einsatz finanzpolitischer Instrumente in der Forschungs- und Entwicklungspolitik. 1978.

Band 11 Hans-Joachim Hauser: Verteilungswirkungen der Staatsverschuldung. Eine kreislauftheoretische Inzidenzbetrachtung. 1979.

Band 12 Gunnar Schwarting: Kommunale Investitionen. Theoretische und empirische Untersuchungen der Bestimmungsgründe kommunaler Investitionstätigkeit in NordrheinWestfalen 1965-1972. 1979.

Band 13 Hans-Joachim Conrad: Stadt-Umland-Wanderung und Finanzwirtschaft der Kernstädte. Amerikanische Erfahrungen, grundsätzliche Zusammenhänge und eine Fallstudie für das Ballungsgebiet Frankfurt am Main. 1980.

Band 14 Cay Folkers: Vermögensverteilung und staatliche Aktivität. Zur Theorie distributiver Prozesse im Interventionsstaat. 1981.

Band 15 Helmut Fischer: US-amerikanische Exporttörderung durch die DISC-Gesetzgebung. 1981.

Band 16 Günter Ott: Einkommensumverteilungen in der gesetzlichen Krankenversicherung. Eine quantitative Analyse. 1981.

Band 17 Johann Hermann von Oehsen: Optimale Besteuerung. (Optimal Taxation). 1982.

Band 18 Richard Kössler: Sozialversicherungsprinzip und Staatszuschüsse in der gesetzlichen Rentenversicherung. 1982.

Band 19 Hinrich Steffen: Zum Handlungs- und Entscheidungsspielraum der kommunalen Investitionspolitik in der Bundesrepublik Deutschland. 1983.

Band 20 Manfred Scheuer: Wirkungen einer Auslandsverschuldung des Staates bei flexiblen Wechselkursen. 1983. 
Band 21 Christian Schiller: Staatsausgaben und crowding-out-Effekte. Zur Effizienz einer Finanzpolitik keynesianischer Provenienz. 1983.

Band 22 Hannelore Weck: Schattenwirtschaft: Eine Möglichkeit zur Einschränkung der öffentlichen Verwaltung? Eine ökonomische Analyse. 1983.

Band 23 Wolfgang Schmitt: Steuern als Mittel der Einkommenspolitik. Eine Ergänzung der Stabilitätspolitik? 1984.

Band 24 Wolfgang Laux: Erhöhung staatswirtschaftlicher Effizienz durch budgetäre Selbstbeschränkung? Zur Idee einer verfassungsmäßig verankerten Ausgabengrenze. 1984.

Band 25 Brita Steinbach-van der Veen: Steuerinzidenz. Methodologische Grundlagen und empirisch-statistische Probleme von Länderstudien. 1985.

Band 26 Albert Peters: Ökonomische Kriterien für eine Aufgabenverteilung in der Marktwirtschaft. Eine deskriptive und normative Betrachtung für den Allokationsbereich. 1985.

Barid 27 Achim Zeidler: Möglichkeiten zur Fortsetzung der Gemeindefinanzreform. Eine theoretische und empirische Analyse. 1985.

Band 28 Peter Bartsch: Zur Theorie der längerfristigen Wirkungen 'expansiver' Fiskalpolitik. Eine dynamische Analyse unter besonderer Berücksichtigung der staatlichen Budgetbeschränkung und ausgewählter Möglichkeiten der öffentlichen Defizitfinanzierung. 1986.

Band 29 Konrad Beiwinkel: Wehrgerechtigkeit als finanzpolitisches Verteilungsproblem. Möglichkeiten einer Kompensation von Wehrungerechtigkeit durch monetäre Transfers. 1986.

Band 30 Woltgang Kitterer: Effizienz- und Verteilungswirkungen des Steuersystems. 1986.

Band 31 Heinz Dieter Hessler: Theorie und Politik der Personalsteuern. Eine Kritik ihrer Einkommens- und Vermögensbegriffe mit Blick auf die Leistungsfähigkeitstheorie. 1994.

Band 32 Wolfgang Scherf: Die beschäftigungspolitische und fiskalische Problematik der Arbeitgeberbeiträge zur Rentenversicherung. Eine Auseinandersetzung mit der Kritik an der lohnbezogenen Beitragsbemessung. 1987.

Band 33 Andreas Mästle: Die Steuerunion. Probleme der Harmonisierung spezifischer Gütersteuern. 1987.

Band 34 Günter Ott: Internationale Verteilungswirkungen im Finanzausgleich der Europäischen Gemeinschaften. 1987.

Band 35 Heinz Haller: Zur Frage der zweckmäßigen Gestalt gemeindlicher Steuern. Ein Diskussionsbeitrag zur Gemeindesteuerreform. 1987.

Band 36 Thomas Kuhn: Schlüsselzuweisungen und fiskalische Ungleichheit. Eine theoretische Analyse der Verteilung von Schlüsselzuweisungen an Kommunen. 1988.

Band 37 Walter Hahn: Steuerpolitische Willensbildungsprozesse in der Europäischen Gemeinschaft. Das Beispiel der Umsatzssteuer-Harmonisierung. 1988.

Band 38 Ulrike Hardt: Kommunale Finanzkraft. Die Problematik einer objektiven Bestimmung kommunaler Einnahmemöglichkeiten in der gemeindlichen Haushaltsplanung und im kommunalen Finanzausgleich. 1988.

Band 39 Jochen Michaelis: Optimale Finanzpolitik im Modell überlappender Generationen. 1989.

Band 40 Bernd Raffelhüschen: Anreizwirkungen der sozialen Alterssicherung. Eine dynamische Simulationsanalyse. 1989.

Band 41 Berend Diekmann: Die Anleihe- und Darlehenstransaktionen der Europäischen Gemeinschaften. 1990.

Band 42 Helmut Kaiser: Konsumnachfrage, Arbeitsangebot und optimale Haushaltsbesteuerung. Theoretische Ergebnisse und mikroökonometrische Simulation für die Bundesrepublik Deutschland. 1990. 
Band 43 Rüdiger von Kleist: Das Gramm-Rudman-Hollings-Gesetz. Ein gescheiterter Versuch der Haushaltskonsolidierung. 1991.

Band 44 Rolf Hagedorn: Steuerhinterziehung und Finanzpolitik. Ein theoretischer Beitrag unter besonderer Berücksichtigung der Hinterziehung von Zinserträgen. 1991.

Band 45 Cornelia S. Behrens: Intertemporale Verteilungswirkungen in der gesetzlichen Krankenversicherung der Bundesrepublik Deutschland. 1991.

Band 46 Peter Saile: Ein ökonomischer Ansatz der Theorie der intermediären Finanzgewalten Die Kirchen als Parafisci. 1992.

Band 47 Peter Gottfried: Die verdeckten Effizienzwirkungen der Umsatzsteuer. Eine empirische allgemeine Gleichgewichtsanalyse. 1992.

Band 48 Andreas Burger: Umweltorientierte Beschäftigungsprogramme. Eine Effizienzanalyse am Beispiel des "Sondervermögens Arbeit und Umwelt". 1992.

Band 49 Jeanette Malchow: Die Zuordnung verteilungspolitischer Kompetenzen in der Europäischen Gemeinschaft. Eine Untersuchung aufgrund einer Fortentwicklung der ökonomischen Theorie des Föderalismus. 1992.

Band 50 Barbara Seidel: Die Einbindung der Bundesrepublik Deutschland in die Europäischen Gemeinschaften als Problem des Finanzausgleichs. 1992.

Band 51 Ralph Wiechers: Markt und Macht im Rundfunk. Zur Stellung der öffentlich-rechtlichen Rundfunkanstalten im dualen Rundfunksystem der Bundesrepublik Deutschland. 1992.

Band 52 Klaus Eckhardt: Probleme einer Umweltpolitik mit Abgaben. 1993.

Band 53 Oliver Schwarzkopf: Die Problematik unterschiedlicher Körperschaftsteuersysteme innerhalb der EG. 1993.

Band 54 Thorsten Giersch: Bergson-Wohlfahrtsfunktion und normative Ökonomie. 1993.

Band 55 Li-Fang Chou: Selbstbeteiligung bei Arzneimitteln aus ordnungspolitischer Sicht. Das Beispiel der Bundesrepublik Deutschland. 1993.

Band 56 Harald Schlee: Einkommensteuerliche Behandlung von Transferzahlungen. Zur Neuordnung der Familienbesteuerung sowie der Besteuerung von Versicherungsleistungen und Sozialtransfers. 1994.

Band 57 Alexander Spermann: Kommunales Krisenmanagement. Reaktionen baden-württembergischer Stadtkreise auf steigende Sozialhilfekosten und Einnahmenauställe (198092). 1993.

Band 58 Otto Roloff / Sibylle Brander / Ingo Barens / Claudia Wesselbaum: Direktinvestitionen und internationale Steuerkonkurrenz. 1994.

Band 59 Claudia Wesselbaum-Neugebauer: Internationale Steuerbelastungsvergleiche. 1994.

Band 60 Stephanie Miera: Kommunales Finanzsystem und Bevölkerungsentwicklung. Eine Analyse des kommunalen Finanzsystems vor dem Hintergrund der sich abzeichnenden Bevölkerungsentwicklung am Beispiel Niedersachsens unter besonderer Berücksichtigung des Landkreises Wolfenbüttel und seiner Gemeinden. 1994.

Band 61 Wolfgang Scherf: Die Bedeutung des kaldorianischen Verteilungsmechanismus für die gesamtwirtschaftlichen Wirkungen der staatlichen Neuverschuldung. 1994.

Band 62 Rainer Volk: Vergleich der Vergünstigungseffekte der verschiedenen investitionsfördernden Maßnahmen. 1994.

Band 63 Hans-Georg Napp: Kommunale Finanzautonomie und ihre Bedeutung für eine effiziente lokale Finanzwirtschaft. 1994. 2., unveränderte Auflage 1994.

Band 64 Bernd Rahmann / Uwe Steinborn / Günter Vornholz: Empirische Analyse der Autonomie lokaler Finanzwirtschaften in der Europäischen Gemeinschaft. 1994. 
Band 65 Carsten Kühl: Strategien zur Finanzierung der Altlastensanierung. 1994.

Band 66 Stephan Boll: Intergenerationale Umverteilungswirkungen der Fiskalpolitik in der Bundesrepublik Deutschland. Ein Ansatz mit Hilfe des Generational Accounting. 1994.

Band 67 Karl Justus Bernhard Neumärker: Finanzverfassung und Staatsgewalt in der Demokratie. Ein Beitrag zur konstitutionellen Finanztheorie. 1995.

Band 68 Christian Haslbeck: Zentrale versus dezentrale Internalisierung externer Effekte bei unvollständiger Information. 1995.

Band 69 Regina Müller: Horizontale oder vertikale Transfers zur Durchsetzung eines horizontalen Finanzausgleichs. 1995.

Band 70 Christian Hockenjos: Öffentliche Sportförderung in der Bundesrepublik Deutschland. Darstellung und finanztheoretische Analyse. 1995.

Band 71 Manfred Rosenstock: Die Kontrolle und Harmonisienung nationaler Beihilfen durch die Kommission der Europäischen Gemeinschaften. 1995.

Band 72 Christian Rüsch: Wohnungsbau- und Wohneigentumspolitik im Rahmen der Einkommensteuer. Eine Analyse unter steuersystematischen, verteilungspolitischen und fiskalischen Aspekten. 1996.

Band 73 Stephan Winters: Die kollektive Vorsorge für den Pflegefall im Alter. Eine Untersuchung am Beispiel der gesetzlichen Pflegeversicherung in den Niederlanden. 1996. 


\title{
Frank Schulz-Nieswandt
}

\section{Stationäre Altenpflege und "Pflegenotstand" in der Bundesrepublik Deutschland}

\author{
Frankfurt/M., Bern, New York, Paris, 1990. 138 S., 14 Abb., 5 Tab. \\ Sozialökonomische Schriften. Bd. 2 \\ Herausgegeben von Bert Rürup \\ ISBN 3-631-42689-5 br. DM 42.--*
}

Die vorliegende Arbeit skizziert den komplexen Prozeß der Präferenzbildung für stationäre Altenpflege und ihrer Inanspruchnahme. Unter Berücksichtigung des sozio-demographischen Problemdrucks sowie durch Rekurs auf ein ganzheitliches Pflegebedarfskonzept wird insbesondere der Frage der De-Institutionalisierung (Substitution stationärer durch - ambulant gestützte - häusliche Pflege) nachgegangen. Im Rahmen einer kritischen Auseinandersetzung mit den Erwartungen der neoklassischen Ökonomik hinsichtlich der Einführung einer Pflege-Sozialversicherung ("moral hazard" - und "Sog "-Effekte) wird eine sozialstaatliche Lösung (Öffentliche Sicherstellung) befürwortet, die die allokativen und distributiven Mängel des bisherigen Systems der Sozialhilfe-Finanzierung beseitigen soll.

Aus dem Inhalt: "Pflegenotstand" und Sozialhilfe-Finanzierung Soziodemographischer Problemdruck - Kritik der De-Institutionalisierungshoffnungen - Determinanten der Heimpflege - Plädoyer für eine sozialstaatliche Problemlösung

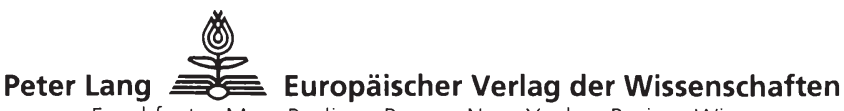

Frankfurt a.M. - Berlin - Bern - New York - Paris - Wien

Auslieferung: Verlag Peter Lang AG, Jupiterstr. 15, CH-3000 Bern 15

Telefon (004131) 9402121, Telefax (004131) 9402131

- Preisänderungen vorbehalten - *inklusive Mehrwertsteuer 
Stephan Winters - 978-3-631-75191-6

Downloaded from PubFactory at 01/11/2019 07:17:47AM

via free access 